\title{
Towards Vertical Impact Analysis of UML Models
}

By:

\section{Tao Yue}

A thesis submitted to the Faculty of Graduate Studies and Research

In partial fulfillment of the requirements for the degree of

Master of Applied Science

Ottawa-Carleton Institute of Electrical and Computer Engineering

Department of Systems and Computer Engineering

Carleton University

Ottawa, Ontario, Canada

July 2006

Copywright (C) 2006 by Tao Yue 


$\begin{array}{ll}\begin{array}{l}\text { Library and } \\ \text { Archives Canada }\end{array} & \begin{array}{l}\text { Bibliothèque et } \\ \text { Archives Canada }\end{array} \\ \begin{array}{l}\text { Published Heritage } \\ \text { Branch }\end{array} & \begin{array}{l}\text { Direction du } \\ \text { Patrimoine de l'édition }\end{array} \\ \begin{array}{l}\text { 395 Wellington Street } \\ \text { Ottawa ON K1A 0N4 } \\ \text { Canada }\end{array} & \begin{array}{l}\text { 395, rue Wellington } \\ \text { Ottawa ON K1A ON4 } \\ \text { Canada }\end{array}\end{array}$

Your file Votre référence ISBN: 978-0-494-18337-3 Our file Notre référence ISBN: $978-0-494-18337-3$

NOTICE:

The author has granted a nonexclusive license allowing Library and Archives Canada to reproduce, publish, archive, preserve, conserve, communicate to the public by telecommunication or on the Internet, loan, distribute and sell theses worldwide, for commercial or noncommercial purposes, in microform, paper, electronic and/or any other formats.

The author retains copyright ownership and moral rights in this thesis. Neither the thesis nor substantial extracts from it may be printed or otherwise reproduced without the author's permission.
AVIS:

L'auteur a accordé une licence non exclusive permettant à la Bibliothèque et Archives Canada de reproduire, publier, archiver, sauvegarder, conserver, transmettre au public par télécommunication ou par l'Internet, prêter, distribuer et vendre des thèses partout dans le monde, à des fins commerciales ou autres, sur support microforme, papier, électronique et/ou autres formats.

L'auteur conserve la propriété du droit d'auteur et des droits moraux qui protège cette thèse. $\mathrm{Ni}$ la thèse ni des extraits substantiels de celle-ci ne doivent être imprimés ou autrement reproduits sans son autorisation.
In compliance with the Canadian

Privacy Act some supporting forms may have been removed from this thesis.

While these forms may be included in the document page count, their removal does not represent any loss of content from the thesis.
Conformément à la loi canadienne sur la protection de la vie privée, quelques formulaires secondaires ont été enlevés de cette thèse.

Bien que ces formulaires aient inclus dans la pagination, il n'y aura aucun contenu manquant.

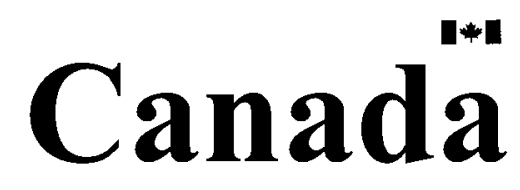




\begin{abstract}
During iterative, UML-based software development, various UML diagrams, modeling the same system at different levels of abstractions are developed. These models must remain consistent when changes are performed. In this context, we refine the notion of impact analysis and distinguish horizontal impact analysis-that focuses on changes and impacts at one level of abstraction, and vertical impact analysis-that focuses on changes at one level of abstraction and their impacts on another level. We propose a vertical impact analysis approach for UML 2.0 models which is based on a careful formalization of changes to those models, refinements which are composed of those changes, and traceability links corresponding to refinements. We show how actual refinements and corresponding traceability links are formalized using the Object Constraint Language. Tool support and an initial case study are also described.
\end{abstract}




\section{Acknowledgments}

First, I would like to sincerely express my thanks to Dr. Briand and Dr. Labiche for their help and guidance throughout my research. I have learnt a great deal from them.

I would like to thank Maged Elaasar for his help, encouragement and patience.

I would like to thank everyone at the SQUALL Lab for mutual support, inspiration and encouragement.

I would like my thesis to be acknowledged in memory of my dear father who is always in my deep heart and with warmest thanks to my mother, my brothers and sisters for their unconditional support, encouragement and love.

A special thanks goes to my husband, Wenjun Bian, for his constant support, care and love. Without him, I could not accomplish this thesis.

Last, but not least, I would like to thank all my friends for their support and encouragement. 


\section{Table of Contents ${ }^{1}$}

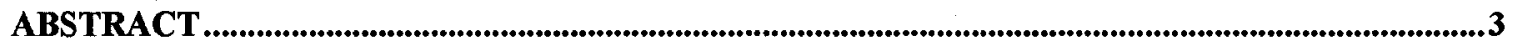

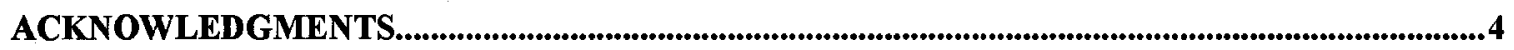

TABLE OF CONTENTS.....................................................................................................................................................5

LIST OF FIGURES ...........................................................................................................................................

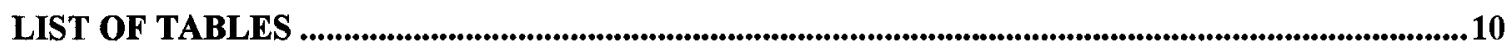

1 INTRODUCTION...........................................................................................................................................11

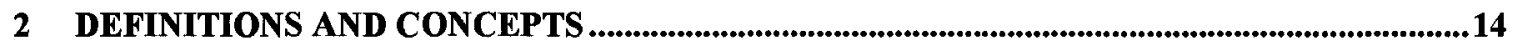

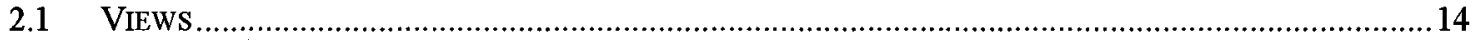

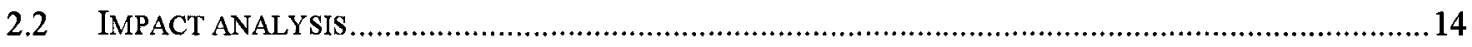

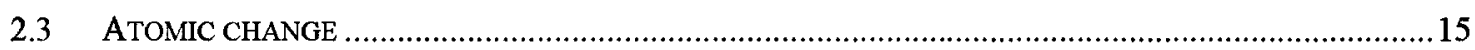

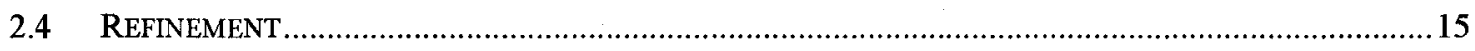

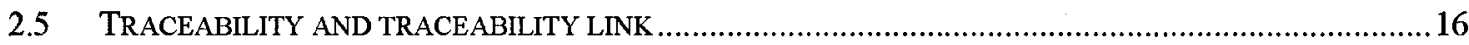

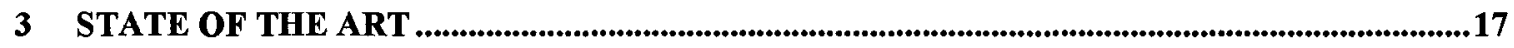

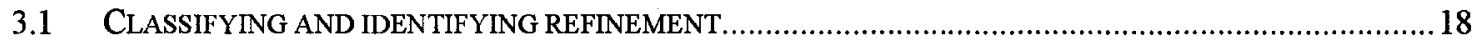

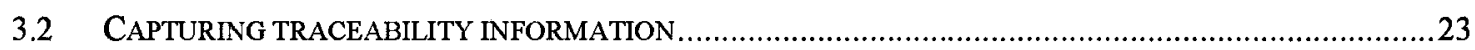

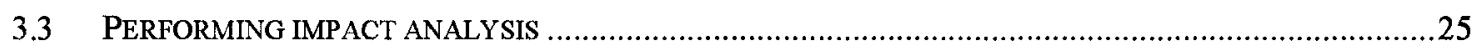

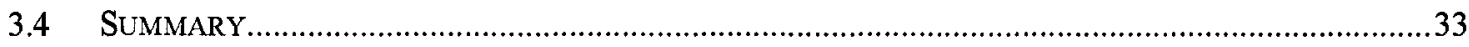

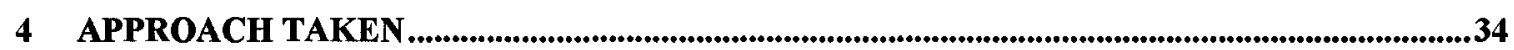

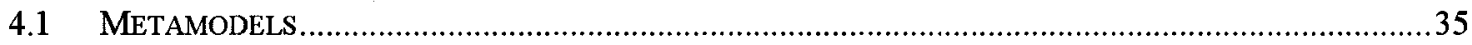

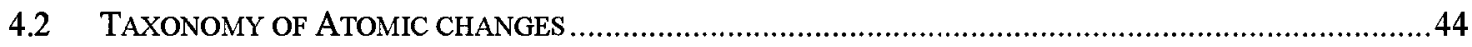

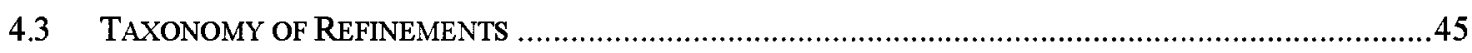

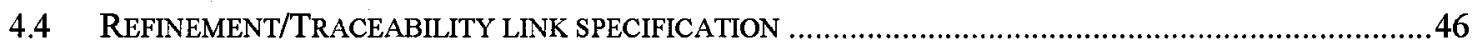

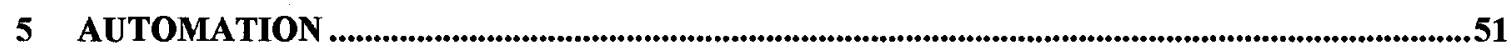

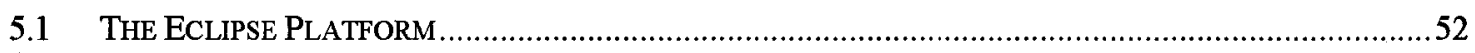

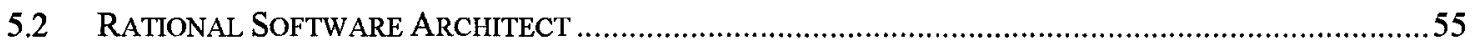

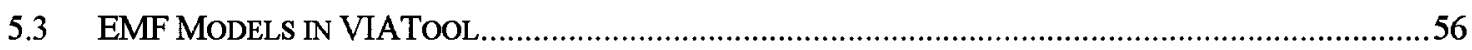

6 CASE STUDY ................................................................................................................................59

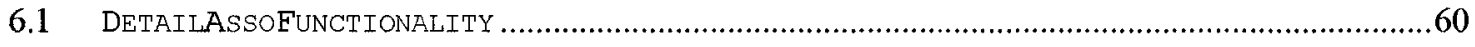

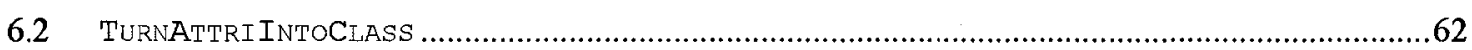

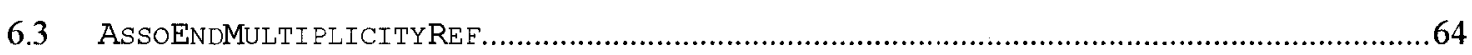

\footnotetext{
${ }^{1}$ A complete table of contents can be found at the very end of the document.
} 


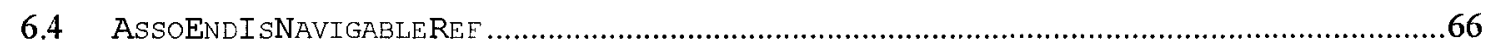

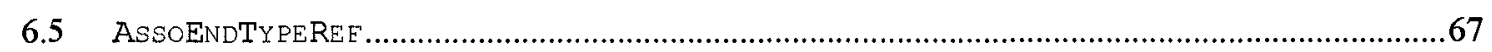

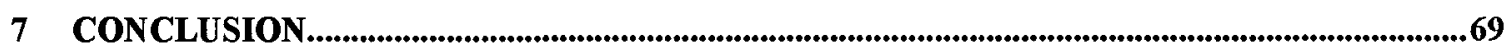

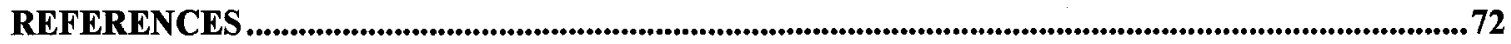

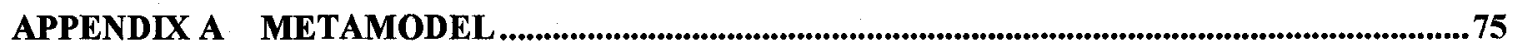

APPENDIX B DICTIONARY FOR METAMODEL

APPENDIX C EXAMPLE ATOMIC CHANGES............................................................................88

APPENDIX D TAXONOMY FOR ATOMIC CHANGES ................................................................95

APPENDIX E TAXONOMY AND FORMALIZATION FOR REFINEMENTS ...........................101

APPENDIX F FORMALIZATION OF PATH..............................................................................191 


\section{List of Figures}

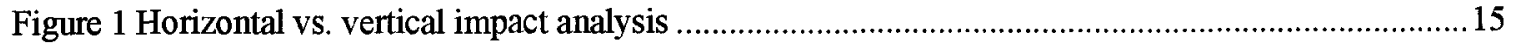

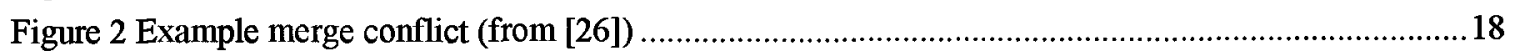

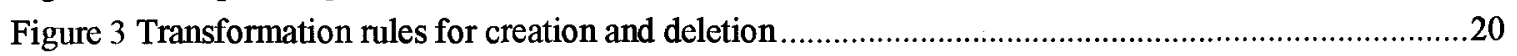

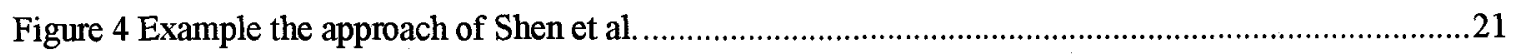

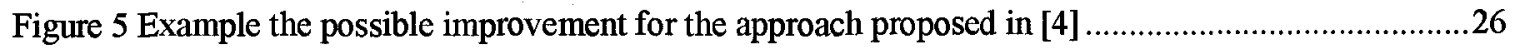

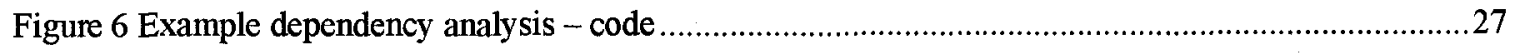

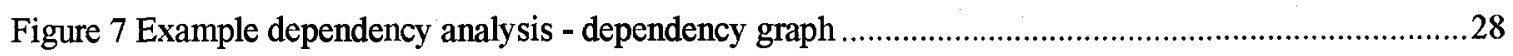

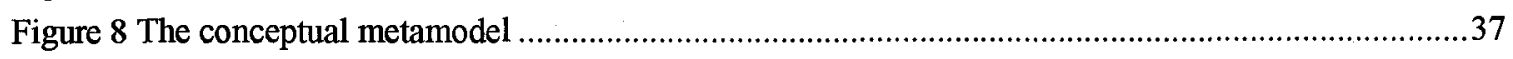

Figure 9 Example composite refinement - original class diagram (from [31]) …....................................38

Figure 10 Example composite refinement - refined class diagram (from [31]) ….....................................38

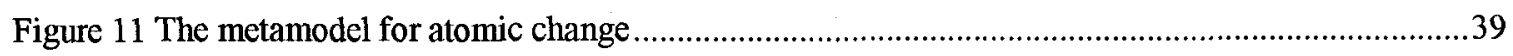

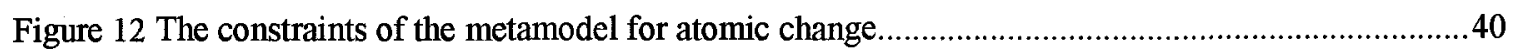

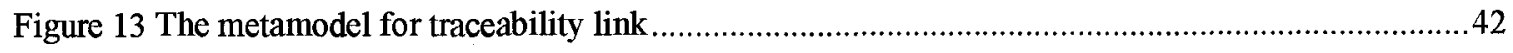

Figure 14 The metamodel for performing vertical impact analysis....................................................44

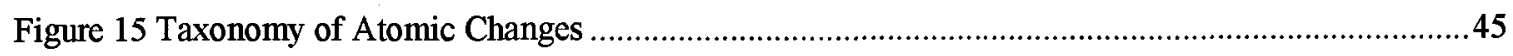

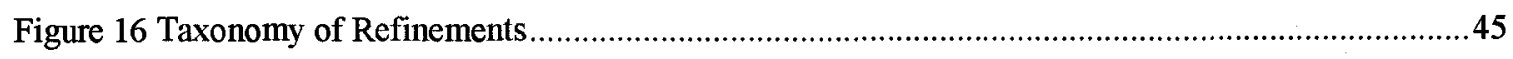

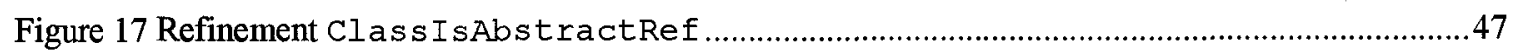

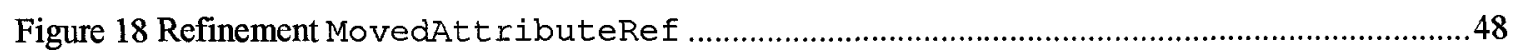

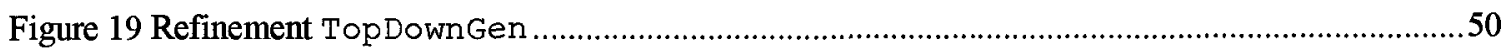

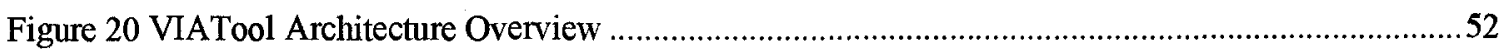

Figure 21 Creating a new refinement repository model .............................................................57

Figure 22 Creating a TopDownGen refinement object ........................................................................57

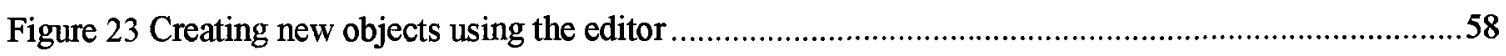

Figure 24 Editing Attributes and Associations using the editor ............................................................58

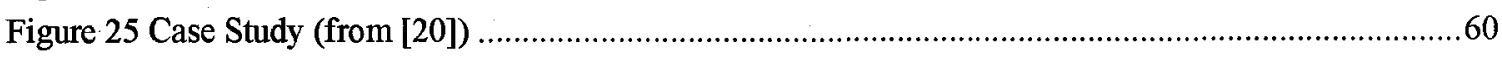

Figure 26 Refinement DetailAs s o Functionality ...............................................................62

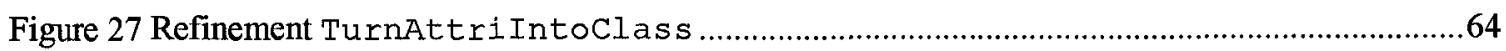

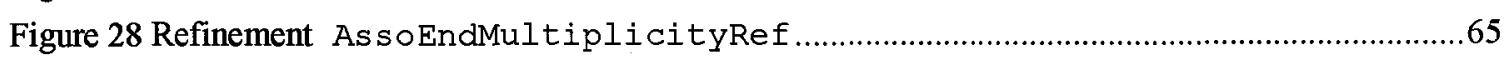

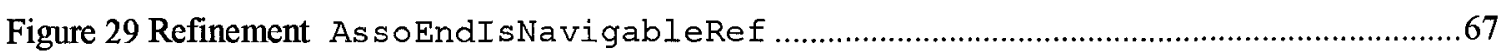

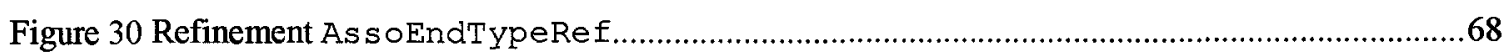

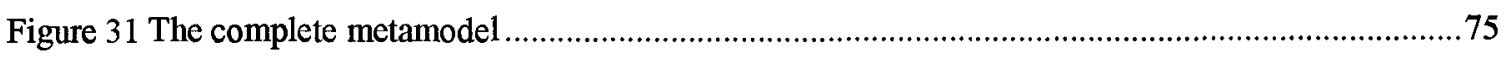

Figure 32 Complete Taxonomy for Atomic Changes - Top Level ...........................................................98

Figure 33 Taxonomy for Atomic Changes of Type ChangedClas s ...................................................99

Figure 34 Taxonomy for Atomic Changes of Type ChangedAttribute ............................................99

Figure 35 Taxonomy for Atomic Changes of Type Changedoperation ..........................................99

Figure 36 Taxonomy for Atomic Changes of Type ChangedAs sociation.........................................99

Figure 37 Taxonomy for Atomic Changes of Type ChangedGeneralization...................................99

Figure 38 Taxonomy for Atomic Changes of Type ChangedInterface ...........................................100

Figure 39 Taxonomy for Atomic Changes of Type ChangedDependency ........................................100

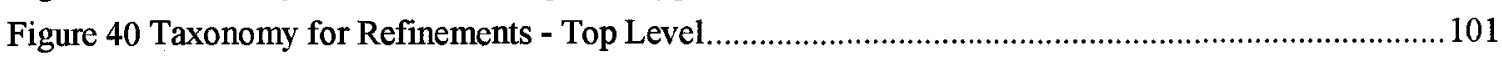

Figure 41 Taxonomy for Refinement of Type Class->Classes+Rels ........................................102 
Figure 42 TopDownGen - Specification 104

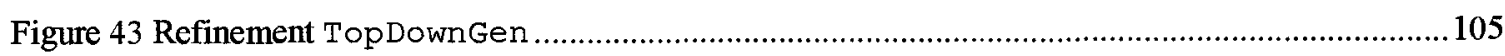

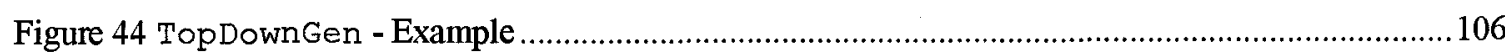

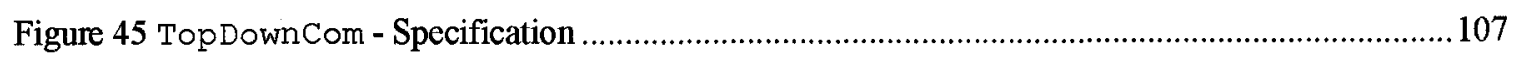

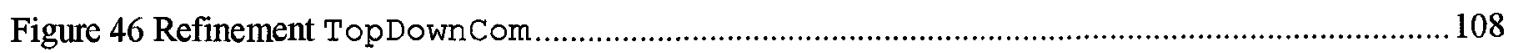

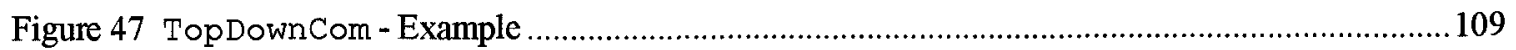

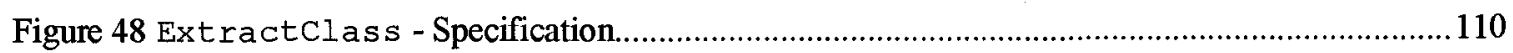

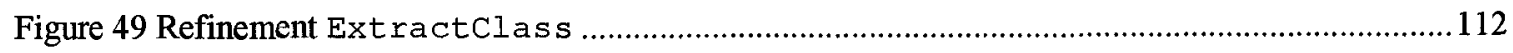

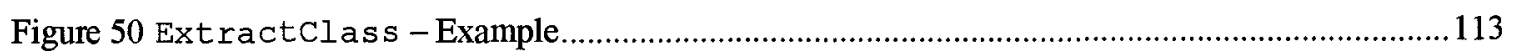

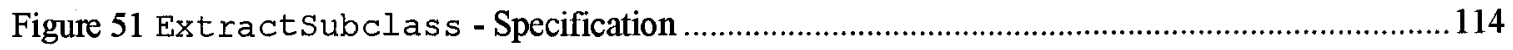

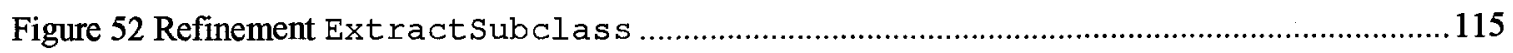

Figure 53 ExtractSubclass - Example......................................................................... 116

Figure 54 TurnAttriIntoClass - Specification ...............................................................117

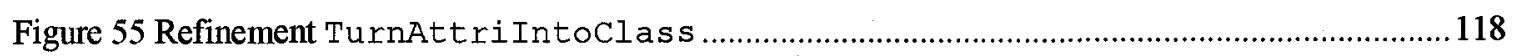

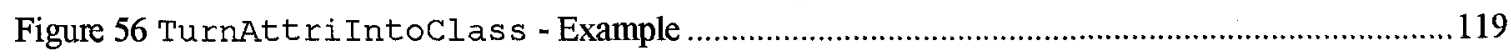

Figure 57 TurnAttriIntoSubclas - Specification . ...................................................... 120

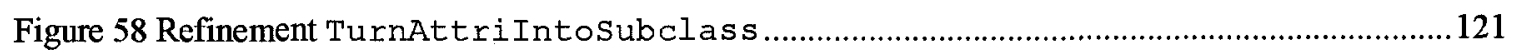

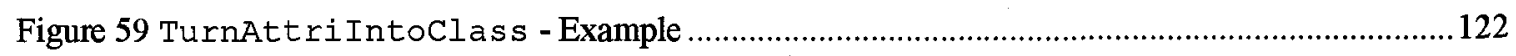

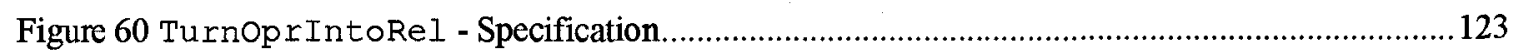

Figure 61 Refinement Turnopr IntoRel ....................................................................... 124

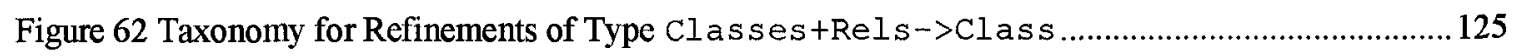

Figure 63 CollapseHierarchy - Specification.....................................................................126

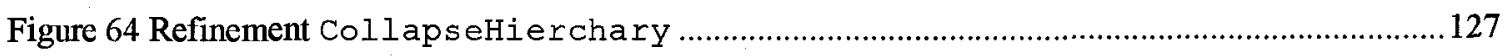

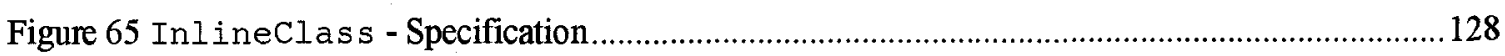

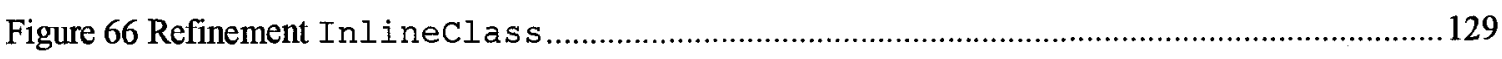

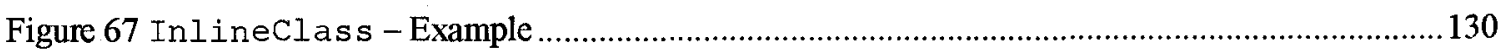

Figure 68 CollapseHiera rchyIntoAttri - Specification ......................................................131

Figure 69 Refinement CollapseHierarchyIntoAttri ...................................................132

Figure 70 Turnclasses +As soIntoAttri - Specification.......................................................133

Figure 71 Refinement TurnClasses +As soIntoAttri .........................................................134

Figure 72 TurnClasses +Rels IntoConst ructor - Specification ............................................135

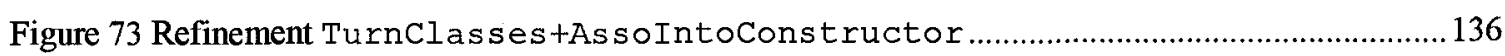

Figure 74 Taxonomy for Refinements of Type Classes+Rels->Classes +Rels ......................137

Figure 75 PullUpAttribute - Specification....................................................................... 138

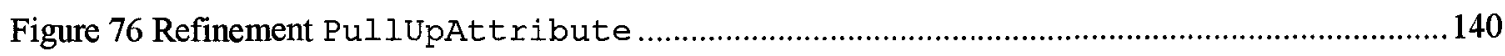

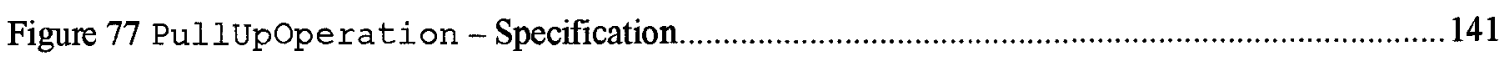

Figure 78 Refinement PullUpoperation .................................................................... 143

Figure 79 PushDownAttribute - Specification................................................................. 144

Figure 80 Refinement PushDownAttribute ...................................................................... 145

Figure 81 PushDownoperation - Specification................................................................ 146

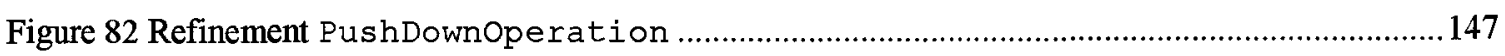

Figure 83 EliminateDupli ByInheritance - Specification...................................................148 
Figure 84 (a) Refinement EliminateDupliByInheritance (part A) ...........................................150

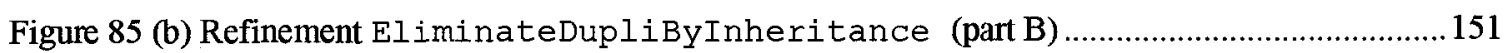

Figure 86 EliminateDupli ByComposition - Specification.....................................................152

Figure 87 (a) Refinement EliminateDupliByComposition (part A) ..........................................154

Figure 88 (b) Refinement EliminateDupliByComposition (part B) .......................................155

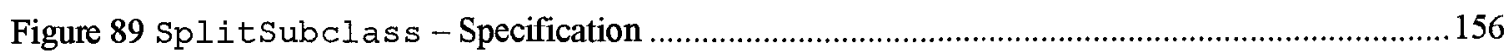

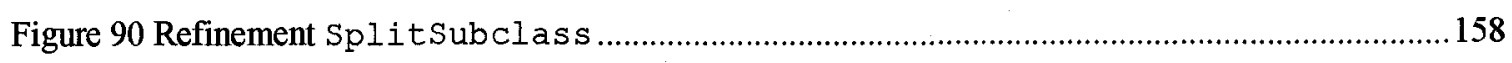

Figure 91 Taxonomy for Refinements of Type Rel->Classes+Rels ............................................159

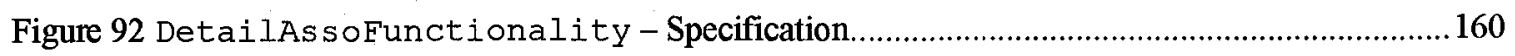

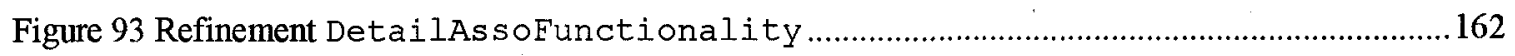

Figure 94 Example DetailAs soFunctionality - the original class diagram...................................163

Figure 95 Example DetailAs soFunctionality - the refined class diagram...................................163

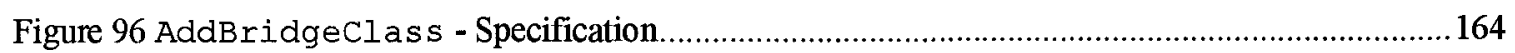

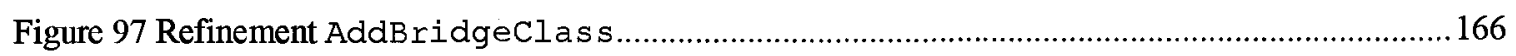

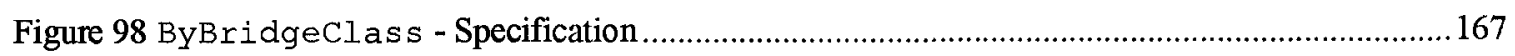

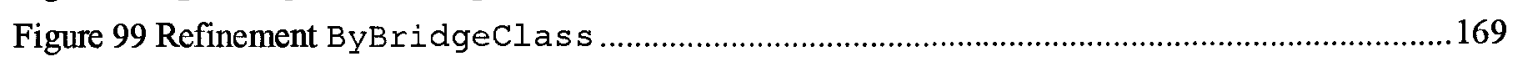

Figure 100 Example ByBridgeClass - the original class diagram ..............................................170

Figure 101 Example ByBridgeclass - the refined class diagram .............................................170

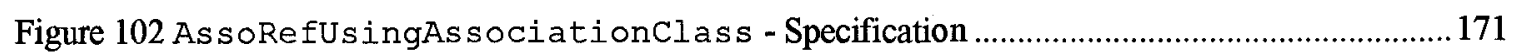

Figure 103 Refinement As soRefUsingAs sociationclass .....................................................172

Figure 104 Taxonomy for Refinements of Type Classes+Rels $\rightarrow$ Rel ............................................173

Figure 105 ReplaceAs sociationClassWithAsso - Specification .............................................173

Figure 106 Refinement ReplaceAs sociationclassWithAs so ....................................................175

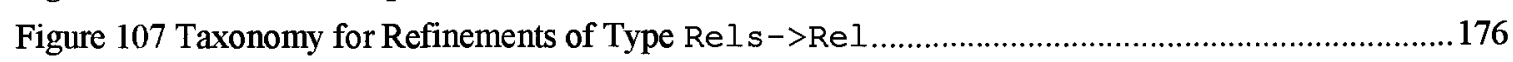

Figure 108 RefAs soesIntoAs so SuperclassAndClient - Specification ......................................176

Figure 109 Refinement RefAs soes IntoAssoSuperclassAndClient (part A) ..........................178

Figure 110 Refinement RefAs soes IntoAs soSuperclas sAndClient (part B)...........................179

Figure 111 Taxonomy for Refinements of Type Rel->Rels ............................................................180

Figure 112 RefAs soIntoAs soes SubclassesAndClient - Specification...................................180

Figure 113 Refinement RefAssoIntoAssoes Subclas sesAndClient (part A) ...........................182

Figure 114 Refinement RefAssoIntoAssoes SubclassesAndClient (part B)...........................183

Figure 115 Taxonomy for Refinements of Type InterfaceRefinement ........................................184

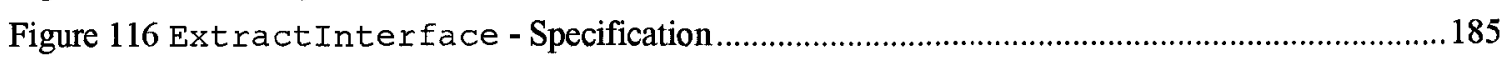

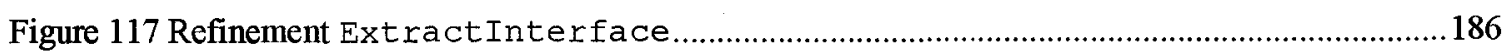

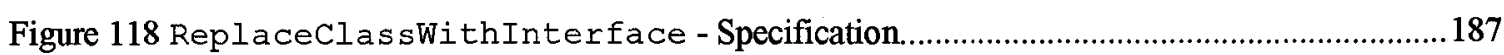

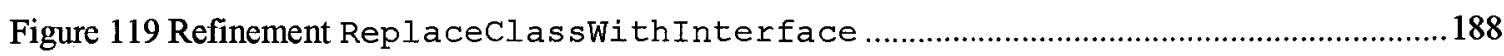

Figure 120 ReplaceInterfaceWithSuperclass - Specification.............................................189

Figure 121 Refinement ReplaceInterfaceWithSuperclass ................................................. 190

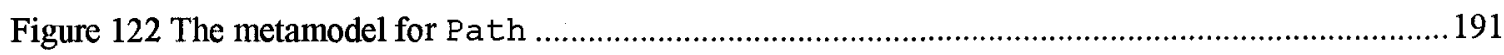




\section{List of Tables}

Table 1 The comparison of existing impact analysis approaches... 


\section{INTRODUCTION}

The use of the Unified Model Language (UML) [29] for complex systems leads to a large number of inter-dependent UML diagrams that have to be consistent, e.g., the operations used in sequence diagrams must be defined in class diagrams. Furthermore, recent development methodologies, such as the Rational Unified Process [19], promote successive modeling iterations evolving and refining models until the final product is complete. As a result, for a specific system, different versions of the same UML models are produced at different levels of abstraction at successive points in time. In the simplest case, one can consider two standard analysis and design abstraction levels [5]. An iterative development process should ensure that these two models remain consistent as they are incrementally refined and changed.

Using and updating these different models should be supported by some tool infrastructure, and one way to cope with the maintenance of such models is to comply with the Model Driven Architecture (MDA) Framework [18]. According to this framework, a platform independent model (PIM) - the analysis model - is (automatically) transformed into a platform specific model (PSM) - the design model, which is itself (automatically) transformed into code. To allow (fully) automated transformations, MDA requires that tools "maintain the relationship between PIM and PSM, even when changes to the PSM are made. Changes in the PSM will thus be reflected in the PIM, and highlevel documentation will remain consistent with the actual code." [18] In practice, when such transformations are not (fully) automated, an essential requirement is that some form of traceability between the models must be created and maintained and support needs to be provided to facilitate the change of a PSM model when its corresponding PIM model is changed.

With the evolution of software systems, UML models undergo changes that address changed requirements and error corrections. If a change takes place at the higher, most abstract modeling level (e.g., analysis model, or original model version), caused for example by changed requirements, it will lead to subsequent changes on model elements 
at lower modeling levels (e.g., design model, or refined model version). Similarly, if change takes place at the lower modeling levels, caused for example by some error correction, it will lead to subsequent changes on model elements at higher modeling levels.

Impact analysis is defined in [2] as the process of "identifying the potential consequences of a change, and estimating what needs to be modified to accomplish that change." In the context of UML-based iterative development, we refine this notion and distinguish Horizontal Impact Analysis (HIA) from Vertical Impact Analysis (VIA). HIA focuses on changes and impacts at one level of abstraction, and corresponds to what people have generally been doing (e.g., [3, 4]), whereas VIA focuses on changes at one level of abstraction and their impacts at another level of abstraction. (This is similar to the notion of horizontal and vertical consistency between models [13].)

Both HIA and VIA require some level of traceability. Traceability is "the ability to trace between software artifacts generated and modified during the software product life cycle." [2] In the case of vertical impact analysis, traceability must exist between model elements at the more abstract (analysis) level and model elements at the more refined (design) level.

In this thesis we describe an approach to support, in a semi-automated way, the vertical impact analysis of UML models and we formalize the notions of traceability (link) and refinement in that specific context. Our approach is motivated by two objectives: (1) Specify, in an unambiguous manner, possible types of refinements and traceability rules, (2) Facilitate its automation based on state-of-the-art, industry-strength modeling technology. An overview of the prototype tool implementing the approach and an initial case study are presented in this thesis as well.

This work's main contribution is a methodology and automation strategy for establishing traceability links between two UML models at different levels of abstraction. Another contribution is that the methodology is formalized using metamodel and constraints, using the UML and the OCL, respectively. This has the advantage of facilitating the automation using available, industry modeling technology. In order to automatically 
identify refinements, we provide a systematic and hierarchical classification for class diagram refinement which allows us to clearly define and formalize class diagram refinements as well as the corresponding traceability links. We made an effort to be as systematic as possible and also describe a mechanism to extend this classification. Another contribution towards an ease of automation is that refinements are (semi)automatically identified instead of requiring that the user explicitly provide them in his/her models. Yet another contribution is that we made a conscious effort to facilitate future extensions. Although this is a first step towards vertical impact analysis, our metamodel allows for new atomic changes, refinements, and UML models to be considered. It also already supports horizontal and vertical impact analysis concepts. 


\section{DEFINITIONS AND CONCEPTS}

This section generally defines terms that will be used in the rest of the thesis. Some of these definitions will be refined in our specific context in subsequent sections.

\subsection{Views}

A view is a representation of an entire system from the perspective of a related set of concerns [25]. UML-based development uses different views to clarify different important aspects of a system. For example, class diagrams, interaction diagrams and statecharts are different views, which are used to describe structural and behavioral aspects of a system.

\subsection{Impact analysis}

Impact analysis can be used for planning changes, making changes and tracing the effect of changes, as a measure of the cost of a change, and to drive regression testing. The major goal of impact analysis is to identify the software artifacts impacted by a set of (proposed) changes.

As we mentioned in Section 1, we refine the notion impact analysis and distinguish horizontal impact analysis (HIA) from vertical impact analysis (VIA): HIA focuses on changes and impacts at one level of abstraction, whereas VIA focuses on changes at one level of abstraction and their impacts at another level of abstraction. This is illustrated in Figure 1 where, for the sake of simplicity, we consider two levels of abstraction. A change to a sequence diagram at the analysis (abstraction) level may impact other analysis diagrams: this is horizontal impact analysis. The same change may also impact model elements at the design (abstraction) level: this is vertical impact analysis. 


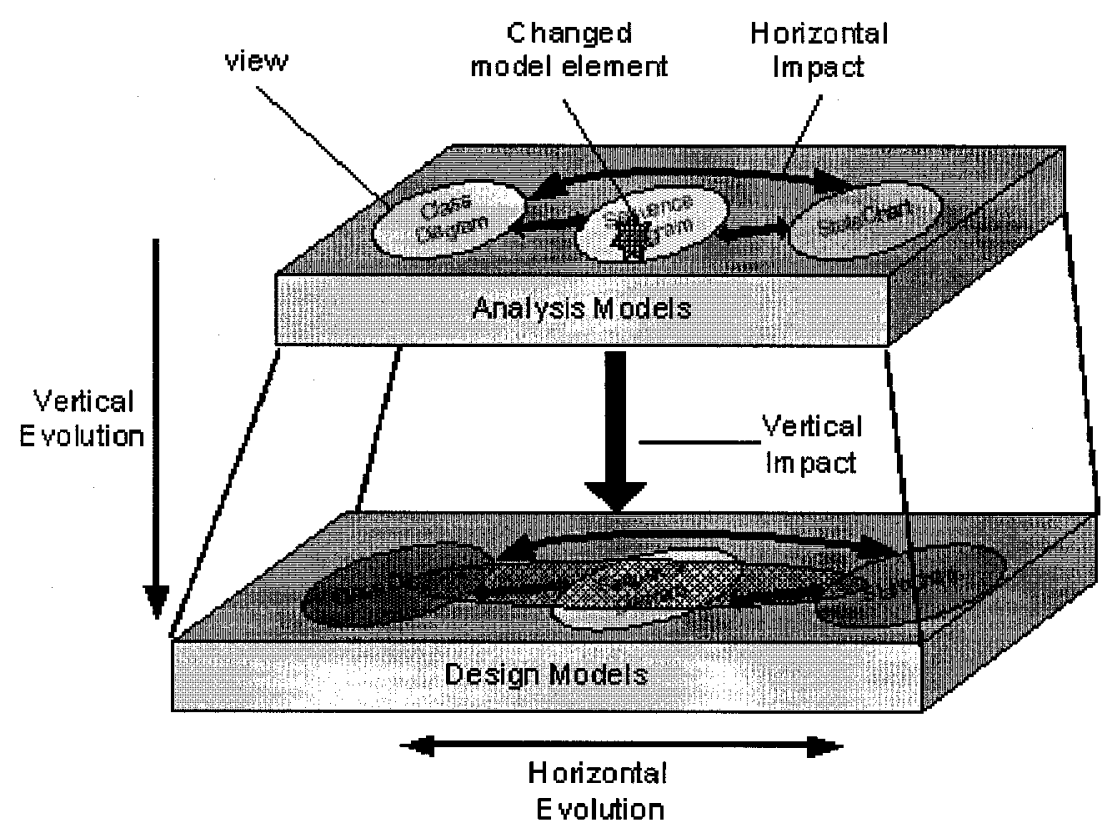

Figure 1 Horizontal vs. vertical impact analysis

\subsection{Atomic change}

An atomic change is a unitary and primitive change applied to a UML model context that cannot be decomposed into smaller atomic changes. For example, "added an association" and "deleted an attribute from a class" are atomic changes. Atomic changes are the elementary steps by which one UML model evolves to another.

\subsection{Refinement}

Refinement is defined as "a software development technique in which data and processing steps are defined broadly at first and then further defined with increasing detail." [24] In the context of UML modeling, we define refinement as means of transforming a more abstract model into a more concrete model. It can be any one of the following:

1. Refining an analysis model into a design model;

2. Refining a version of a UML model into the next version of the same UML model; 
3. Refining a version of a class diagram (or sequence diagram, etc.) into the next version of the same class diagram (or sequence diagram, etc.);

4. Refining a version of part of a class diagram (or sequence diagram, etc.) into the next version of the same part of the class diagram (or sequence diagram, etc.).

\subsection{Traceability and traceability link}

As we mentioned in Section 1, traceability must be established between model elements at different levels of abstraction. In our context, we define a traceability link as a relationship that describes the traceability connection between a model element of one UML model and a model element of another UML model. In order to identify traceability links, we must either capture (during model modifications) or determine (by comparison of models) the intent of the designer that lay behind changes when models are refined. In other words, traceability links are derived from the identification of refinements. 


\section{STATE OF THE ART}

Since vertical impact analysis is an important activity of model evolution, automatically supporting it is required for UML CASE tools. However, almost all existing tools contributing to software evolution are primarily targeted to source code. Model evolution (supported by UML CASE tools, for example) is much less sophisticated. With the growing application of UML, the techniques and tools for dealing with the model evolution are urgently needed.

Recall from Section 2 that, in order to perform vertical impact analysis, atomic changes must be recorded during the process of refining UML models. Then a refinement is derived from these atomic changes. Again, traceability links are established since we assume we specified which traceability links should be established for a specific refinement beforehand. Last, vertical impact analysis can be performed after traceability links are established.

In this section of the thesis we address three important areas where vertical impact analysis requires support:

1. Classifying and identifying refinement.

2. Capturing traceability information.

3. Performing vertical impact analysis.

There are many texts and papers published describing model evolution, however, capturing traceability information for the purpose of performing vertical impact analysis on UML models has not been directly addressed in the research literature. Besides, most of existing approaches classify refinements at the level of atomic changes, rather than more complex levels such as the refinements derived from several atomic changes. As a result, they are not amenable to capturing the user's intent.

This section of the thesis details the latest research in automated support of vertical impact analysis. It provides a structured and comprehensive survey on the three issues discussed above. Section 3.4 summarizes some important issues that have to be addressed. 


\subsection{Classifying and identifying refinement}

In order to establish traceability links between model elements of two UML models at different levels of abstraction, we have to classify and identify refinements which are possibly made by the user in order to refine a UML model into another one. How to classify and identify refinements determines which kind of traceability information we can derive and which kind of vertical impact analysis result we can provide to the user.

\subsubsection{Mens et al. - Automating Support for Software Evolution in UML}

Mens et al. [26] propose an approach to detect conflicts between parallel evolutions of the same UML model, such as a merge conflict which may be due to different designers independently and concurrently making changes to the same software artifact. Four different types of refinements are considered: Addition, Removal, Connection and Disconnection. The UML metamodel is modified and specific stereotypes are defined to explicitly represent modifications.

Figure 2 is adapted from [26]. This example is a merge conflict which arises from the combination of independent modifications leading to undesired interactions and occurs when two parallel modifications are made to the same model element.

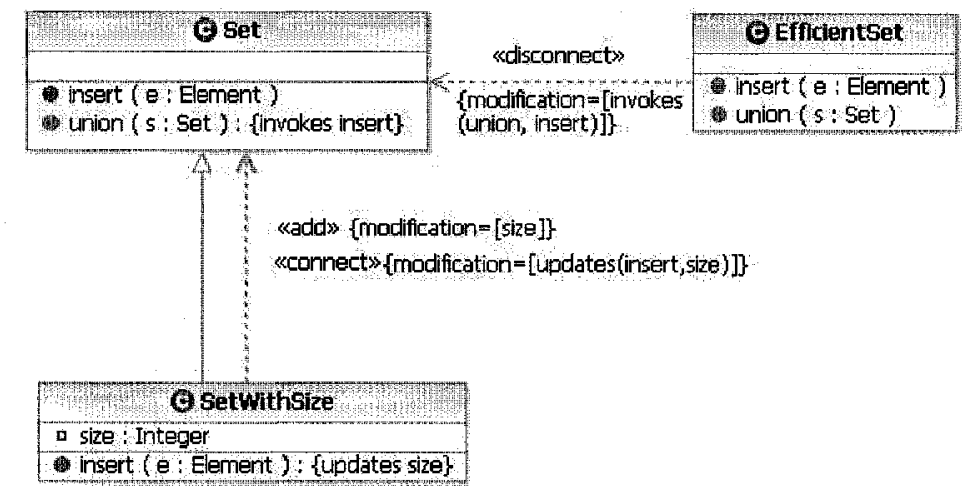

Figure 2 Example merge conflict (from [26])

Parallel modification 1: Set is specialized into Setwithsize which overrides insert() operation so that it additionally accesses and updates attribute size each time a new element is added to the set. The dependency relationship (dashed line) specifies that 
Setwithsize is incrementally obtained from set by adding attribute size (Addition) and accessing the attribute size from operation insert() (Connection).

Parallel modification 2: Set evolves into new version Efficientset by removing the call to insert() in union() for efficiency reasons. The refinement type is Disconnection.

To find out if upgrading set into Efficientset leads to unexpected results, we need to know whether setwithsize is still a valid and meaningful specialization of Efficientset. Since originally the invocation of union() in Setwithsize leads to an indirect update of size, this will not be the case anymore if we substitute set with Efficientset because operation union() in Efficientset does not invoke insert () anymore. This is called "inconsistent operation conflict" in [26], and it occurs each time one modification is a Disconnection of an operation invocation (e.g., in Figure 2, operations insert () and union() are disconnected in Efficientset) and the other modification is a Connection involving the same operation (e.g., in Figure 2, operation insert () is connected to attribute size).

The major problem with this approach is that designers are required to explicitly and manually represent their modifications in UML diagrams (e.g., dependency relationships and predefined stereotypes are used). This, however, is a significant burden to designers and eventually clutters diagrams. This approach still has other limitations, for example, an interface can only be evolved into an interface, rather than a class.

\subsubsection{Engels et al. - Consistency-Preserving Model Evolution through Transformation}

Engels et al. [12] propose an approach to preserve consistency during the evolution of UML-RT [32] models, a variation of UML specifically dedicated to real-time systems. Three kinds of modifications, namely Creation, Deletion and Update are identified. The paper focuses on four main model elements of UML-RT models, namely capsules, port, connectors, and protocols. Each atomic change is specified by two patterns representing the situation before and after the change. For example, Figure 3 describes two atomic 
changes excerpted from [12], which assumes two capsules $A$ and $B$ with an unconnected port each (left part of Figure 3) and creates a connector between them (right part of Figure 3). This is atomic change Creation. When read from right to left, the atomic change is Deletion as it removes an existing connector from its ports.

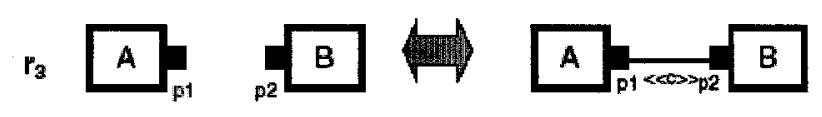

Figure 3 Transformation rules for creation and deletion

After identifying possible evolution steps by means of atomic changes, for a certain property possessed by the original model, like deadlock freedom or protocol consistency, conditions of the application of atomic changes, which ensure the modified model enjoys a similar property, are formulated. Thanks to a (partial) mapping from UML-RT to Communicating Sequential Processes (CSP), the approach is able to identify under which conditions, modifications of these model elements maintain consistency. The paper restricts its analysis, for instance to non-hierarchical UML-RT models. It also mentions that atomic changes representing elementary evolution steps may be combined in various ways to achieve more complex changes; however no concrete approach is proposed. Another difficulty is that it is required to keep consistency between models and their CSP specification.

\subsubsection{Shen et al. - Extending the UML Metamodel to Support Software Refinement}

Shen et al. [33] propose an approach to support UML class diagram refinement by extending the UML metamodel. Stereotypes are used to represent the refinement explicitly. The paper only concerns the relationship refinement in a class diagram, as it is assumed that a relationship refinement in a class diagram plays a crucial role and that in most cases a class diagram refinement can be inferred from the corresponding relationship refinement. Four types of relationship refinements are defined as follows:

- Generalization abstraction: a generalization relationship is refined into two generalization relationships plus a new class. 
- Bidirectional association abstraction: a bidirectional association is refined into two bidirectional associations plus a new class.

- Unidirectional association abstraction: a unidirectional association is refined into two unidirectional associations plus a new class.

- Association composition abstraction: a composition association is refined into two composition associations plus a new class or a composition association is refined into one composition association, plus a generalization relationship, and a new class.

Each relationship is qualified with a predefined stereotype which distinguishes the original relationships from the refined relationships. Figure 4 is an example excerpted from this paper. The $<<$ Refined_Asso $>>$ and the $<<$ Refining_Asso $>>$ are predefined stereotypes which are used to qualify the associations.

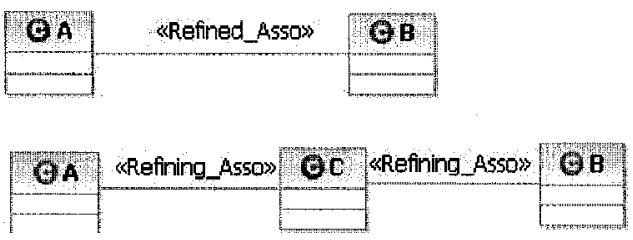

Figure 4 Example the approach of Shen et al.

We can see that this approach just supports one kind of refinements of class diagram: relationship refinement. There are still a lot of other important types of refinements. Another difficulty of this approach is that the refinement must be explicitly represented using predefined stereotypes.

\subsubsection{Hnatkowska et al. - Refinement relationship between collaborations}

Hnatkowska et al. [15] propose a classification of nine class diagram refinements:

1. Adding a class;

2. Adding a class property (attribute or operation);

3. Modifying a class attributes;

4. Modifying a class operations;

5. Splitting a class into two classes with association between them; 
6. Introducing a successor of a class (i.e., two classes with association between them);

7. Adding an association;

8. Modifying of association;

9. Introducing an intermediate class.

$1,2,3,4,7$ and 8 are actually atomic changes, basic kinds of refinement. 5, 6 and 9 are more complex. These structural refinements are then used to specify behavioral refinements in collaboration diagrams and how the collaboration refinements relate to the corresponding structural refinements. Only a few simple refinements are presented though, and it is not clear (at least this is not described) how those refinements can be detected by a tool.

\subsubsection{Pons and Kutsche - Traceability Across Refinement Steps in UML modeling}

Pons and Kutsche [30] present a number of refinements for class diagram and use cases. Class diagram refinements are of the following types:

- Attribute Refinement: adds a new attribute or replaces an existing attribute with another one.

- Operation Refinement: adds a new operation or replaces an existing operation with another one.

- Refining by Specification: adds generalization relationship.

- Refining by Composition: adds composite association.

Use Case refinements are of the following types:

- Refining by Action Decomposition: refines one single action into several actions to see more detail.

- Refining by Specialization: a use case specializes a more general one.

The paper utilizes a UML metamodel element, namely Abstraction, to link refined and refinement elements, thus allowing establishment of a (possibly formal) mapping. All of these refinements are required to be explicitly and manually represented using stereotype 
$<<$ refine $>>$. This paper doesn't provide a complete set of refinements for either class or use case diagrams.

\subsubsection{Discussion}

There are several issues about the existing approaches on the classification of refinement. Firstly, most of existing works do not support complex refinements which should be derived from more than one atomic changes, except for Hnatkowska et al. [15] who present several such refinements. Secondly, each work just provides a partial classification of refinements. For example, Shen et al. [33] only covers relationship refinements in class diagrams.

In terms of the identification of refinements, most of existing works require to explicitly represent refinements. Several works use stereotypes to do so. This kind of approach significantly increases the user's involvement and burden. It is therefore unlikely to be appliable in practice.

\subsection{Capturing traceability information}

In order to perform vertical impact analysis, traceability information between two UML models must be captured. Traceability links can be built after the identification of refinements which determine which traceability links must be established between which model elements involved in a given refinement.

\subsubsection{Letelier - A Framework for Requirements Traceability in UML-based Projects}

Letelier [21] proposes a framework for the specification of traceability links between high-level requirements and UML models. These traceability links between different types of specifications enable the verification that the system functionality covers the stakeholder's expectation, and impact analysis when requirements change. Although primarily intended to support stakeholders in tracing high-level (textual) requirements to various UML models during initial development phases, the framework also allows the definition of traceability links between UML model elements. 
In this work, a metamodel is proposed, in which classes represent entity types (e.g., requirements, UML models and stakeholders), and associations represent types of traceability links (e.g., stakeholder 'responsibleOf' requirement, and requirement 'traceTo' UML model). Stereotypes are used to explicitly represent traceability links.

The major difficulty with this approach is that most of the traceability links should be established manually, even though the approach supports 'implicit traceability links' which can be derived from several existing, manually established traceability links. Vertical impact analysis can be performed based on these traceability links; however it is too coarse to perform meaningful vertical impact analysis between model elements of two model versions.

\subsubsection{Judson et al. - Supporting Design by Pattern-based Transformation}

Judson et al. [17] present an approach for specifying pattern-based transformations of UML models consisting of class and interaction diagrams. These transformations are specified as metamodels, which indicate, for example, newly introduced classes and existing classes removed by the transformation. Further constraints are defined along with the transformation metamodels, which are used to specify constraints on source and target model elements, and relationships that must be held between source and target model elements. The Abstract Factory pattern is used to illustrate the approach. The paper focuses on the specification of transformations at the metamodel level. How to derive traceability links is not addressed. However it is easy to understand that traceability links can be automatically established since one knows how a model is transformed.

The major difficulty of this kind of transformation-based approach is that the user is requested to select which specific transformation to apply and the model elements to which the transformation is applied.

\subsubsection{Discussion}

In terms of capturing traceability information, the existing works require to explicitly and manually establish or represent traceability links with the exception of the 
transformation-based approach proposed in [17]. This is a serious drawback of these approaches. Another important issue is that some works do not explicitly indicate and formalize which kinds of traceability information should be captured during the refinement process.

\subsection{Performing impact analysis}

Most of impact analysis techniques rely on a graph representation of software artifacts (the nodes) and their dependencies (the edges). First of all, a graph is used to describe the relationships between software artifacts in an intuitive and visual way. Secondly the graph representation can be analyzed using existing techniques and algorithms from graph theory. The main issue with graph-based approaches is that the time complexity of the algorithm tends to grow exponentially with the number of edges. So the efficiency of these techniques may become problematic on large problems.

In this section, several existing approaches are introduced first. Then these approaches are compared in section 3.3.5 in which a set of criteria are defined and comparison results are presented in a table.

\subsubsection{Briand et al. - Automated Impact Analysis of UML Models}

In [4], an approach is proposed to support to the identification of changes made to UML model elements and the impact of these changes on other model elements. The approach is decomposed into four steps: (1) automatically detect and classify changes across different versions of UML models, (2) verify the consistency of changed diagrams, (3) perform an impact analysis to determine the potential side effects of changes in the design, (4) prioritize the results of impact analysis according to the likelihood of occurrence of predicted impacted elements. This paper also proposes an experimental method to evaluate the effectiveness of the impact analysis. A prototype tool iACMTool was developed to assess the feasibility and practicality of the approach.

The systematic approach proposed in [4] focuses on horizontal impact analysis. Before performing horizontal impact analysis, changes across different versions of UML models 
are detected automatically. A change taxonomy is formalized under the form of a tree which contrains ninety-seven (97) leaf changes. Each leaf change of the change taxonomy corresponds to one change detection rule. For example, "AddedAssociation" is a change, whose definition is that "in the changed model version there exists an association relationship that does not exist in the original version." [4] In order to perform horizontal impact analysis, 97 impact analysis rules are defined corresponding to the leaf changes.

The impact analysis rules defined in [4] could be extended in order to get more precise and/or complete horizontal and vertical impact analysis results. Figure 5 presents an example where we assume that the association subAsso has been added (this is a change of type "AddedAssociation"). The changed element is the subAsso and, according to the impact analysis rules proposed in [4], the impacted elements obtained after performing once horizontal impact analysis are the classes SubclassB and Client. However, it is likely that the association superAsso between the classes Superclass and Client may be impacted. If there is no relationship between the classes SubclassA and Client, then it is not necessary to have the association superAsso anymore since classes SubclassB and $\mathrm{client}$ are connected by the association subAsso. So we can obtain a more accurate initial set of impacted elements: the classes Superclass, SubclassB, Client and the association superAsso.

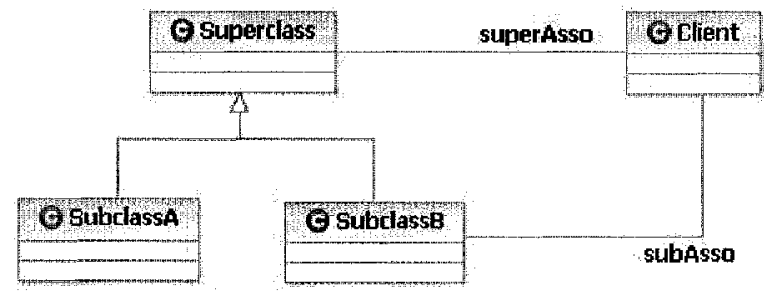

Figure 5 Example the possible improvement for the approach proposed in [4]

\subsubsection{Li and J. Offutt - Algorithmic Analysis of the Impact of Changes to Object- Oriented Software}

A code-based impact analysis approach is proposed in [22, 23]. It suggests a detailed analysis of the changes, organized in a change taxonomy, to precisely study how changes 
propagate (if they do). This paper presents algorithms to analyze the potential impacts of changes to object-oriented software, taking into account encapsulation, inheritance, and polymorphism. This approach is graph-based and an object-oriented data dependency graph is created to support the impact analysis. This impact analysis technique includes a set of algorithms to calculate the change impact according to change impact rules defined beforehand.

All dependencies are stored in a directed dependency graph, where each node represents a program entity (e.g., variable, module and data type) and edges represent the different kinds of dependencies such as operation calls.

Figure 6 and Figure 7 present a simple example taken from [23] to explain how changes propagate among class members. Figure 6 shows a piece of code and Figure 7 presents its corresponding dependency graph. ClassA has six class members: A_methI(), A_meth2 (), A_meth3(), A_field1, A_field2, and A_field3. As shown in Figure 7, method A_meth2() references A_field1, A_field2, A_field3, and A_meth1(), data member $A_{-} f i e l d 1$ is defined by both data member $A_{-} f i e l d 2$ and $A_{-}$field3. Data member $A_{-}$field2 is defined by $A_{-}$methl(). Assume that $A_{-}$methl is changed and triggers change propagation, it will impact $A_{-}$meth2() and A_field2 according to the dependency graph. A_meth2() and A_field2 are directly impacted because of the change of $A_{-} m e t h 1()$. A_field1 and A_meth2() are indirectly impacted because of the indirect impact on $A_{-}$field2.

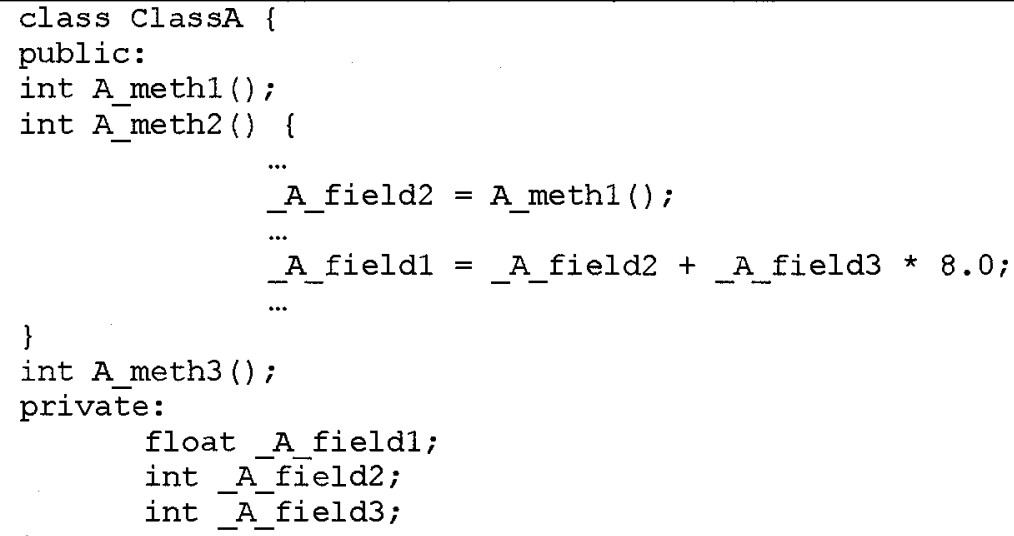

Figure 6 Example dependency analysis - code 


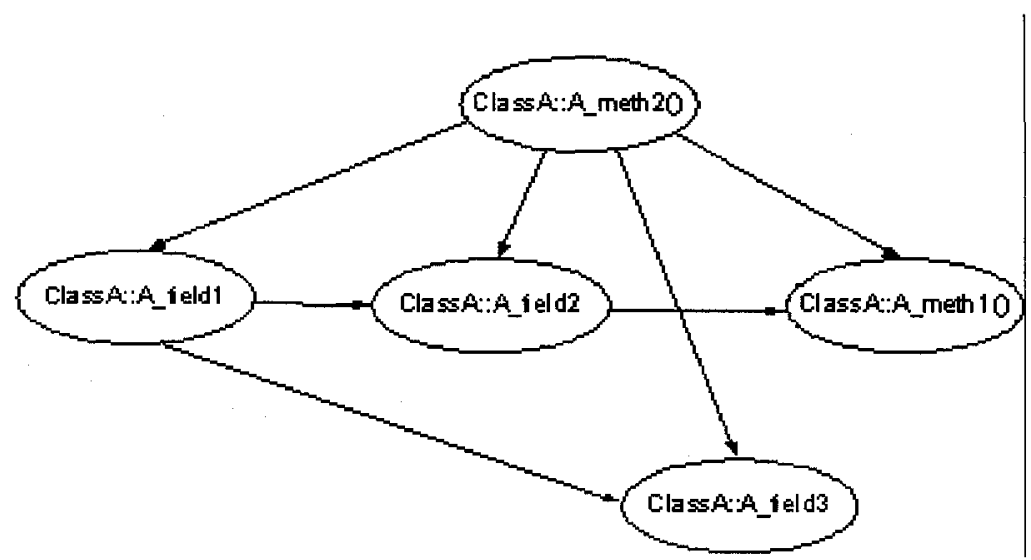

Figure 7 Example dependency analysis - dependency graph

\subsubsection{Mens and D'Hondt - Automating Support for Software Evolution in UML}

An automated approach is proposed to support UML model evolution in [26]. It is claimed to cover all the model evolution activities such as consistency checking, impact analysis, conflict detection and traceability management. However, only the evolution activity of conflict detection is explained in this paper. It does not explicitly address impact analysis.

\subsection{4 von Knethen et al. - QuaTrace: A Tool Environment for (Semi-)Automatic Impact Analysis Based on Traces.}

A tool environment is proposed in [34] for (semi-) automatic impact analysis based on traceabilities. The tool environment is built on two existing tools: RequisitePro (requirement management tool) and Rhapsody (UML case tool). In this approach, because UML model elements are transformed and represented as shadow-requirements (natual language), a lot of information in the UML models is lost, especially relationships among model elements. Besides, traceability links between requirements and UML model elements must be manually established. Name tracing is used to automatically establish implicit traceability links. Based on these traceability links, impact analysis can be performed. Three levels of impacts are defined: primary impacts which are all documentation entities that have to be changed, secondary impacts which are all requirements that have to be changed with a high probability, and tertiary impact which 
are all documentation entities that might be changed. For each impact, simple impact analysis rules are defined.

\subsubsection{Comparison}

The comparison approach proposed in [1] is adapted for our use. Six evaluation criteria are defined:

\section{- Artifact Of Change}

It defines the artifacts for the impact analysis such as UML model or code.

\section{- Change Specification}

It is how the change is specified for the impact analysis approach.

\section{- Intermediate Model}

It is used to define the objects and relationships (or dependencies) the approach uses to accomplish impact analysis. For example, dependency relationships can be modeled by a dependency graph.

- Impact Model

It defines the rules or embedded assumptions reflecting the semantics about what affects what. For example, when one class is changed, we need to follow the rules (impact model) to derive impacted elements.

\section{- Impact Approach}

It implements the impact model. It defines how objects and intermediate models are represented, how impact rules are captured, or the specific search algorithms used to find impacted artifacts.

\section{- Distance Measure}

It is a way to determine the distance of other potential impacts from the original impact or the distance between a changed element and potentially impacted element.

Existing approaches are compared according to the above criteria and results are presented in Table 1. The following part of the section discusses the comparison results.

In terms of the change specification, three different ways are used to classify changes. In [4], 97 fine-grained change categories are specified using OCL expressions. For example, 
"changed sequence diagram view - Added message" is a change category. There are three change actions: add, delete and change. The work in [26] defines 4 primitive evolution contracts: addition, removal, connection and disconnection, and 2 composite evolution contracts: promotion and sequentialisation. This approach requires the user to explicitly and manually represent the evolution contracts. The user's involvement is as a result substantial. Changes are classified and defined in $[22,23]$. These changes are finegrained, for example "Change scope: Private->Public" is a change on a data member. In [34], the approach doesn't need to classify changes since it is very coarse-grained and doesn't support the automatic detection of changes.

In terms of the intermediate model, the UML metamodel and OCL are used by some approaches for impact analysis. In [4] the UML metamodel is used to help determine the possible changes that can occur. In [26], the authors extend the UML metamodel and uses UML extension mechanisms: stereotypes to incorporate evolution contracts in UML. In [34], the documentation model is used to represent documentation entities and their relationships. Besides, relationship and tracing guidelines are derived from the documentation model to support establishing traces of requirements items and the tool relies on these traces to perform impact analysis.

In terms of the impact model, a set of impact analysis rules are defined using OCL expressions in [4], and each impact analysis rule corresponds to each change category in the taxonomy. Each impact analysis rule is a specification of how to derive collections of elements that are potentially impacted by a particular change. Although in [26] the approach could be adapted to impact analysis, due to a lack of detailed information provided in this paper, we can not compare it with [4]. The work in [22, 23] defines incomplete change impact rules. In [34], an analysis algorithm is used to perform impact analysis.

A distance measure is defined in [4] and [22, 23]. In [4] the distance between a changed element and a given impacted element is defined at the number of impact analysis rules that had to be invoked to identify this impacted element. It implies all the impact analysis rules have the same weight. The proposed approach in [34] classifies the impacted 
elements into three types: primary impacts, secondary impacts and tertiary impacts (see Section 3.3.4 for details).

Note that the work in [4] and [22, 23] focuses on horizontal impact analysis. Vertical impact analysis, although this terminology is not used by these authors, is suggested in [26]. No precise vertical impact analysis approach is described though. Even though [34] presents an approach to maintain traceability, it is restricted to traceability between highlevel textual requirement descriptions and use cases (and use case descriptions). 


\begin{tabular}{|c|c|c|c|c|c|c|c|}
\hline Approach & Artifact Of Change & Change Specification & $\begin{array}{l}\text { Intermediate } \\
\text { Model }\end{array}$ & Impact Model & Impact Approach & HIA/VIA $^{2}$ & $\begin{array}{l}\text { Distance } \\
\text { Measure }\end{array}$ \\
\hline [4] & UML models & $\begin{array}{l}\text { 1.Change taxonomy } \\
\text { 2.Change detection rules } \\
\text { OCL expressions }\end{array}$ & $\begin{array}{l}\text { 1.UML metamodel } \\
\text { 2.OCL }\end{array}$ & $\begin{array}{l}\text { Impact analysis } \\
\text { rules }\end{array}$ & $\begin{array}{l}\text { Prerequisites: } \\
\text { 1.Consistency } \\
\text { checking } \\
\text { 2.Change detection } \\
\text { 3.Impact analysis } \\
\text { 4.Prioritization of } \\
\text { impacts }\end{array}$ & $\mathrm{HIA}$ & Yes. \\
\hline [26] & UML models & $\begin{array}{ll}\text { 1.Primitive } & \text { evolution } \\
\text { contracts } & \\
\text { 2.Composite } & \text { evolution } \\
\text { contracts } & \\
\text { 3. User-defined } & \text { evolution } \\
\text { contracts } & \\
& \\
\text { Stereotypes + OCL }\end{array}$ & $\begin{array}{l}\text { 1. UML metamodel } \\
\text { 2. OCL }\end{array}$ & $\begin{array}{l}\text { N/A } \\
\text { (Extendable) } \\
\text { Evolution } \\
\text { contracts }\end{array}$ & N/A (Extendable) & $\begin{array}{l}\text { VIA } \\
\text { (Extendabl } \\
\text { e) }\end{array}$ & $\begin{array}{l}\text { N/A } \\
\text { (Extendable) }\end{array}$ \\
\hline [34] & $\begin{array}{l}\text { 1.High-level } \\
\text { documentation } \\
\text { entities(requirement, } \\
\text { system components). } \\
\text { 2.Low-level types of } \\
\text { documentation } \\
\text { entities (use case, } \\
\text { system funtion). }\end{array}$ & No classification. & $\begin{array}{l}\text { 1.Documentation } \\
\text { model } \\
\text { 2. Relationship } \\
\text { guidelines } \\
\text { 3.Tracing } \\
\text { guidelines }\end{array}$ & $\begin{array}{l}\text { Analysis } \\
\text { algorithm }\end{array}$ & $\begin{array}{l}\text { 1.Build up } \\
\text { traceability among } \\
\text { requirement items. } \\
\text { 2.Based on } \\
\text { established traces to } \\
\text { derive impacted } \\
\text { elements. } \\
\text { 3.Distance measure is } \\
\text { given. }\end{array}$ & VIA & No. \\
\hline$[22,23]$ & Code & $\begin{array}{l}\text { Change category: } \\
\text { 1.Changes on Methods } \\
\text { 2.Changes on Data } \\
\text { Member } \\
\text { 3.Others Changes } \\
\text { Textual description. }\end{array}$ & $\begin{array}{l}\text { 1.Control Flow } \\
\text { Graphs (CFGs) } \\
\text { 2.Data Flow Graphs } \\
\text { (DFGs) } \\
\text { 3.Object-oriented } \\
\text { data dependency } \\
\text { graphs (OODDGs }{ }^{4} \text { ) }\end{array}$ & $\begin{array}{lr}\text { Change impact } \\
\text { rules } & \text { and } \\
\text { algorithms }\end{array}$ & $\begin{array}{l}\text { 1.Converts CFGs and } \\
\text { DFGs to OODDGs. } \\
\text { 2.Apply algorithms } \\
\text { to find all impacted } \\
\text { elements. } \\
\text { 3. Recursively apply } \\
\text { algorithm. }\end{array}$ & HIIA & $\begin{array}{l}\text { Yes. } \\
\text { (implicitly }\end{array}$ \\
\hline
\end{tabular}

Table 1 The comparison of existing impact analysis approaches

${ }^{2}$ HIA stands for horizontal impact analysis. VIA stands for vertical impact analysis.

${ }^{3}$ Relationships guidelines are derived from the documentation model for each relationship defined in the documentation model. The tracing guidelines give additional information to each relationship guideline. They are all used to establish traces among all documentation entities. After all these traces are established, then it is straightforward to perform impact analysis using existing tools.

${ }^{4}$ In an OODDG, the nodes represent data items, such as classes, class members, variables and constants. The edges represent dependencies among these data items.

${ }^{5}$ The paper did not mention any distance measure, but it is obvious that the approach supports the definition of distance measure. 


\subsection{Summary}

In this section, we summarize important issues regarding existing approaches in terms of capturing traceability information and performing vertical impact analysis.

1. Most of existing approaches require to explicitly represent refinements. For example, stereotypes are used in [26] and [33] to explicitly represent refinements. In other words, users are requested to manually and explicitly represent the refinements while making changes to the models. The major disadvantage of these approaches is a significant overhead on the user's part and little automation. Another disadvantage is that the model becomes increasingly cluttered because of the extra information of capturing traceability information.

2. Most existing approaches do not support complex refinements which should be derived from several atomic changes rather than a single atomic change. The resulting problem is that the user's intent can not be captured at a higher level than atomic changes. Even though some approaches support more complex refinements, a systematic classification is not provided.

3. Most of existing approaches do not provide a systematic classification of refinements.

4. Most of existing approaches do not indicate or formalize which kinds of traceability information should be captured during the refinement process.

5. Some vertical impact analysis approaches focus on source code rather than UML models. Others focus on horizontal impact analysis rather than vertical impact analysis. Certain approaches are too coarse-grained to be adapted to the vertical impact analysis of UML models. There is no method that is specifically addressing the vertical impact analysis of UML models. 


\section{APPROACH TAKEN}

Our objective is to perform vertical impact analysis of UML 2.0 models as automatically and efficiently as possible. To do so, we propose an approach which involves horizontal impact analysis and traceability information. First, atomic changes, which are the elementary steps by which one model evolves into another, are identified automatically. Second, refinements are derived from the identified atomic changes, thus capturing the user's intent at a higher level of abstraction than atomic changes. Third, traceability links are established between model elements of UML model versions automatically (e.g., from analysis model elements to design model elements), based on the identified refinements, to keep track of the user's intent. Finally, with two UML model versions, the corresponding traceability links, and a HIA approach in hand, VIA can be performed to answer the question: what is the impact on the refined model of changes to the original model? We intend to automate the above four activities as much as possible, without requiring the designer to explicitly specify refinements (as in [26] for instance). Though the user's input might be necessary for some decisions, we aim at minimizing the occurrence of such user queries. In order to automatically identify refinements, we provide a systematic and hierarchical classification for class diagram refinement. We made an effort to be as systematic as possible and also describe a mechanism to extend this classification.

It is important to note that, according to the four activities discussed above, a change to a model can be used to either identify a refinement and thus traceability links or perform HIA and/or VIA (once traceability links have been identified). The question is then to be able to distinguish between the two during the software development process. As a simplifying working assumption, and given that we intend to automate VIA and HIA in the context of a UML case tool such as Rational Rose Architect, we will assume the following scenario:

1. The designer working on a set of UML models tells the case tool when s/he starts refining those models. At this point in time, the case tool can refer to these models 
as models at the first level of abstraction (say, analysis) and record modifications for the purpose of identifying refinements and traceability links.

2. When the refinement activity ends, the designer informs the case tool: the recording ends. At this point in time, the current UML models are considered as models at the second level of abstraction (say, design) by the tool. Refinements and traceability links have been identified.

3. Any modification to a model at either of the two abstraction levels then triggers some HIA and VIA. For instance, a modification to a model at the first level of abstraction triggers HIA (to identify the impacted elements at the same level of abstraction) and then VIA (to identify the impacted elements at the second level of abstraction).

Whether this simplifying assumption as to how HIA and VIA fit in a software development life cycle will be the subject of future investigations.

In the following, we formalize the notions of atomic change, refinement, traceability link, vertical impact analysis and horizontal impact analysis, by means of metamodels (Section 4.1). We then present taxonomies of atomic changes and refinements for class diagrams (Sections 4.2 and 4.3). Last, we show how we specify refinements and the corresponding traceability links (Section 4.4).

\subsection{Metamodels}

In this section, we present the different concepts of atomic changes, atomic and composite refinements, and traceability links under the form of metamodels. In order to keep the discussion focused, the metamodel is presented in a piecewise manner. New classes and relationships specific to each step are highlighted. The whole metamodel is given in Appendix A for reference. A dictionary describing each metaclass, its attribute(s) and association(s) is provided in Appendix B for reference. 


\subsubsection{Conceptual metamodel}

The class diagram in Figure 8 illustrates the main concepts of our approach, the conceptual metamodel, which is used to present the relationships of the important concepts introduced in Section 2.

A UMLModel is composed of instances of UMLDiagram which can be a ClassDiagram, or any other valid UML diagram. A UMLDiagram is composed of instances of Element (i.e., modelElements), which is one of the meta-classes of the UML 2.0 metamodel [27]. This class is the bridge between our metamodel and the UML 2.0 metamodel. A UMLModel is associated with instances of Refinement by two associations since a model can be refined (rolename toRefinedModel) and at the same time be the refinement of another model (rolename tooriginalModel). A refinement is between the original model (rolename originalModel) and the refined model (rolename refinedModel). Instances of TraceabilityLink should be established for each Refinement instance. How to perform vertical impact analysis based on the established traceability links is presented in Section 4.1.5.

A refinement is either an AtomicRefinement, corresponding to a series of Atomichanges, or a CompositeRefinement composed of AtomicRefinements and/or other CompositeRefinements. Detailed description of atomic and composite refinements will be given in Section 4.1.2, along with examples. We will see in Section 4.2 and 4.3 that AtomicChange and AtomicRefinement are the roots of hierarchies (taxonomy) of atomic changes and refinements, respectively.

Notice that the analyzed models may not contain enough information to identify a refinement, in which case the user's help is requested (class UserHelp). For a particular refinement which requires the user's help, we define specific format of user's help such as yes-no or multiple choices questions in order to minimize the user's involvement. 


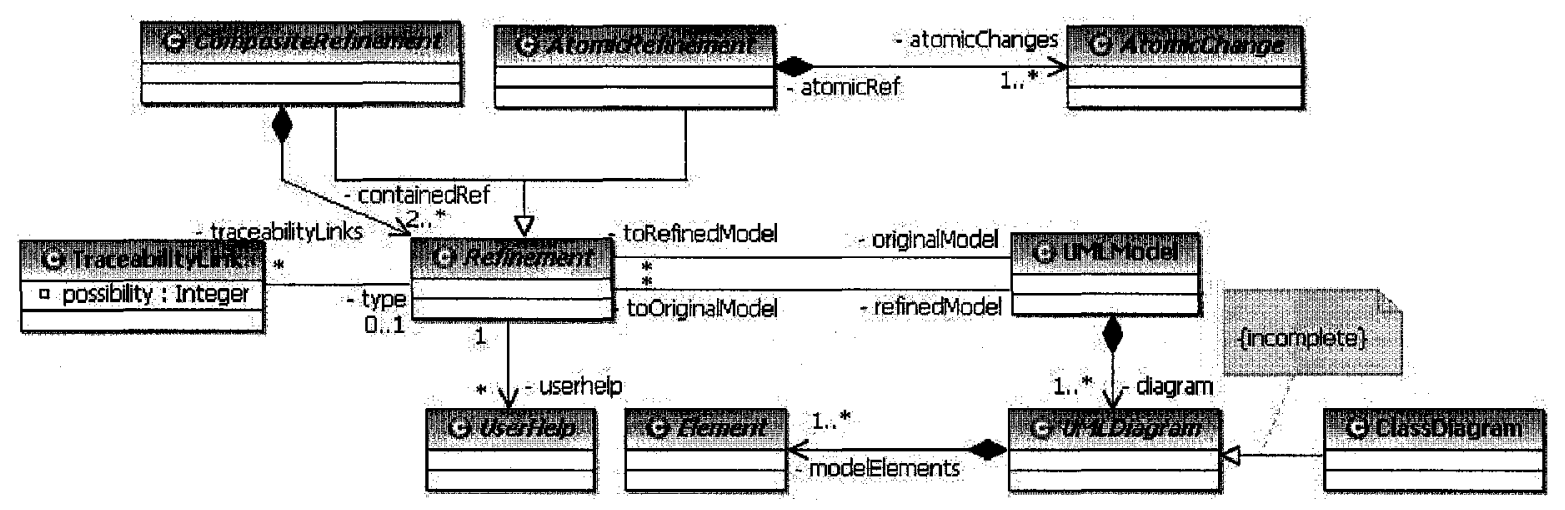

Figure 8 The conceptual metamodel

\subsubsection{Metamodel for refinement}

As shown in Figure 8, an AtomicRefinement is derived from a group of AtomicChanges that occur together. It can not be decomposed into other refinements. For example, refining a class by extracting some of its attributes into a new class which becomes the subclass of the class being refined is an atomic refinement. This refinement is derived from a group of atomic changes: "adding a new class", and "moving an attribute from a class into another", and "adding a generalization". Notice that an atomic refinement may be derived from a single atomic change, in which case the atomic refinement is straightforward.

Notice that the composite design pattern is used to model refinements. A Compositerefinement consists of more than one Refinements which can be AtomicRefinements or CompositeRefinements. The refinements grouped into a composite refinement are between the same original and refined models. In other words, we can write the following OCL constraints:

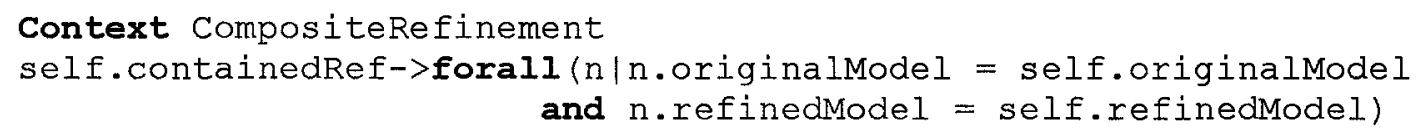

Figure 9 and Figure 10 show a composite refinement example, which is adapted from [31]. Firstly, class ManagementDepartment of the original class diagram is refined into 
class ManagementDepartment which aggregates classes ClientManager, LoanManager and AccountManager. Because of this refinement, it is necessary to refine the association keeps between the classes ManagementDepartment and Account in the original class diagram into the association between the classes AccountManager and Account in the refined class diagram. These two atomic refinements, namely TopDownGen and RelocateAssociation (Appendix E.1.1 and Appendix D), are grouped together as a composite refinement.

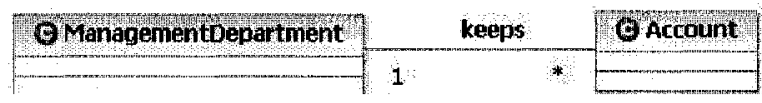

Figure 9 Example composite refinement - original class diagram (from [31])

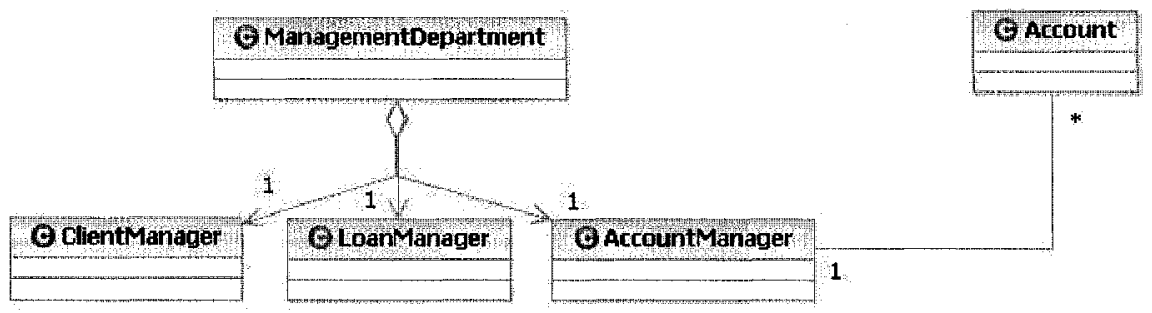

Figure 10 Example composite refinement - refined class diagram (from [31])

\subsubsection{Metamodel for atomic change}

The class diagram in Figure 11 illustrates the metamodel for atomic change. An AtomicChange affects a model element (instance of Element) and is further described by a ChangeDescription. We consider four different kinds of AtomicChanges, as defined in enumeration AtomicChangeTypes: changed, moved, deleted, and added. The last two are self-explanatory: the added (deleted) element is the affectedElement in the refined (original) model. An element is moved when its location changes, e.g., an operation is moved from a class to another. For a moved element, affectedElement represents the element in the original version of the model, whereas targetLocation or diagramLocation associations from class ChangeDescription describe the new location. In the case the moved element belongs to another model element (e.g., an operation belonging to a class is moved), targetLocation is to be used. If instead the moved element belongs to a model (e.g., a class belongs to a class diagram), 
diagramLocation is to be used. A changed element occurs, for instance, when a named element changes name, e.g., an attribute name changes. The name change is then recorded in attributes beforevalue and aftervalue of class ChangeDescription. Obviously beforeValue and aftervalue can not be same. This constraint is formalized using the following OCL expressions.

This discussion of the different kinds of atomic changes typically means that not every instance of Figure 11 is legal, i.e., that there exist constraints. Figure 12 presents those constraints on classes ChangeDescription and AtomicChange, specified using OCL expressions. These constraints are based on the conceptual metamodel (Figure 8) and the metamodel for atomic changes (Figure 11).

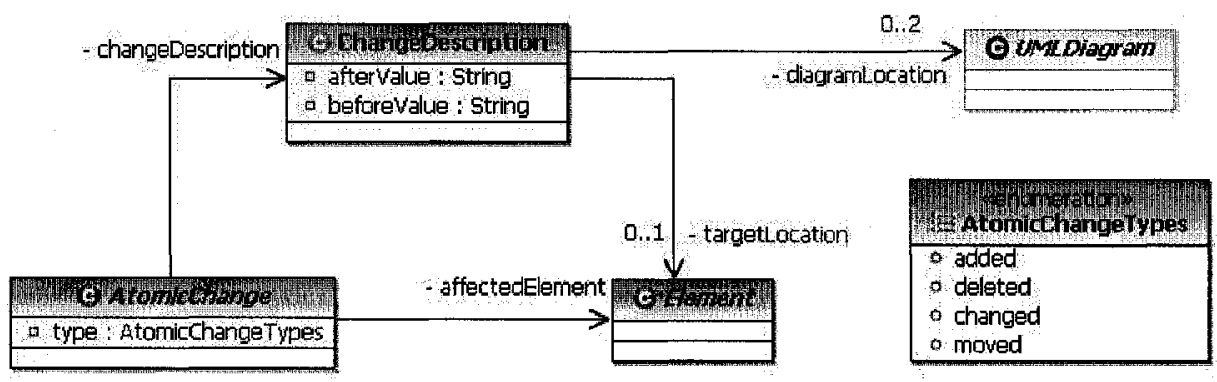

Figure 11 The metamodel for atomic change

In Appendix $\mathrm{C}$, a list of example atomic changes is presented along with a description of the ChangeDescription and affectedElement links of each atomic change.

These notions are consistent with the compare\&merge facility which is available in Rational Software Architect (RSA) [16]. The compare\&merge facility is indeed the mechanism we intend to rely on for the detection of model changes to identify refinements. 


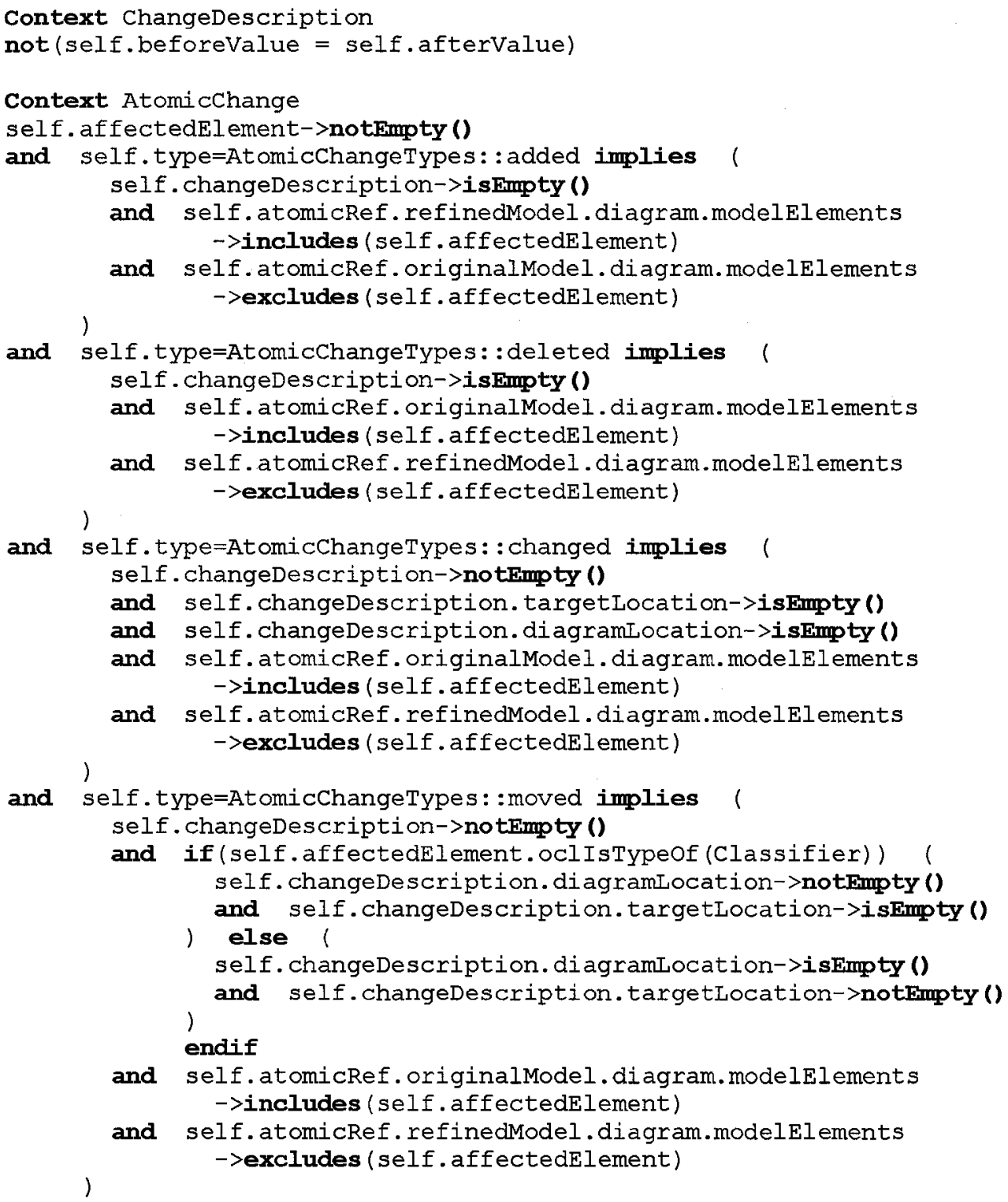

Figure 12 The constraints of the metamodel for atomic change

\subsubsection{Metamodel for traceability link}

A traceability link between two model elements which belong to different UML models can be represented by a tuple of the form (Eo, Er, type, possibility) where Eo is a model element of the original model, Er is a model element of the refined model, type is the type of the traceability link (i.e., corresponding to a particular refinement), and possibility is a percentage which represents the likelihood that there is a traceability 
link. Sometimes, according to the information available in models (e.g., a group of atomic changes, refinement identified and two UML models), it is not possible to guarantee at $100 \%$ that there is a refinement, so we assign a percentage to the traceability link which denotes the confidence that this is indeed a refinement. For example, one possible refinement is to merge a superclass and a subclass together when they are not very different. In order to identify this refinement, we have to compare attributes and operations of the merged class (i.e., a class of the refined model) and the deleted class (i.e., the class of the original model) which could be the superclass or subclass. The extent to which such a mapping of attributes and operations can be established indicates the likelihood of the refinement.

We use an example to show how to use the above tuple format to represent traceability links. Class $A$ is refined by adding a new attribute newAttri (we call this refinement AddedAttributeRef): let us refer to this new version of $A$ as $A 1$. Then class $A$ is refined by making it an abstract class (we call this refinement ClassIsAbstractRef): let us refer to this new version of $A$ as $A 2$. We can use the above tuple format to represent the traceability information between classes A, A1 and A2: (A, A1, AddedAttributeRef, $100 \%)$, and (A1, A2, ClassisAbstractRef, 100\%).

The concept of traceability link is modeled using the class diagram in Figure 13. Instances of Traceabilitylink are established for each Refinement instance, between the elements in the original model that are being refined (association to Element with rolename origin) and the elements of the target model that are the refinements (association to Element with rolename target). An element in one model (i.e., the original model or the refined model) can be connected to an element in another model (i.e., the refined model or the original model) via one or many traceability links. An element in one model (i.e., the original model or the refined model) can be connected to one or many elements in another model (i.e., the refined model or the original model). The origin and target of a traceability link are model elements of the original and refined models, respectively, of the corresponding refinement. In other words, we can write the following OCL constraints: 


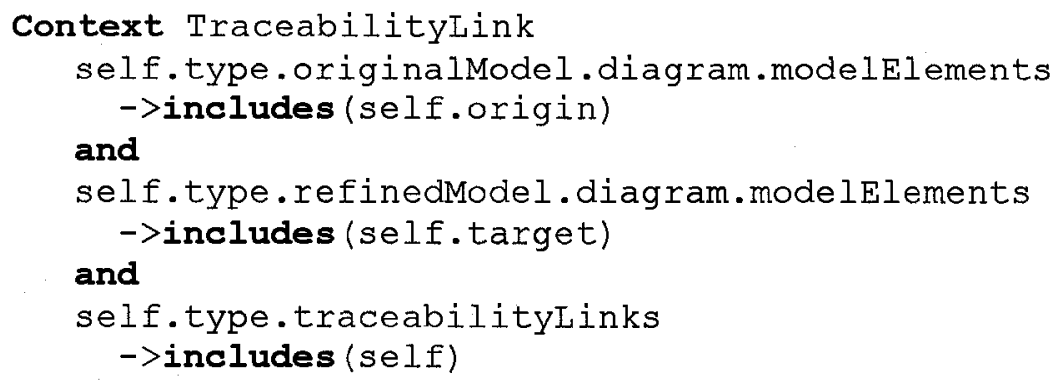

When traceability links can be established between a series of models, we can derive traceability links between the elements of the first (most abstract) model and the elements of the last (most refined) model, these links being modeled as instances of class DerivedTraceabilityLink. For instance, in the abstract example above, we can derive a traceability link between A and A2 from the two traceability links between A and A1, and between $A 1$ and $A 2$. Notice that class $A 1$ is the target of the traceability link between $A$ and $A 1$, but also the origin of the traceability link between $A 1$ and $A 2$. We can therefore write the following OCL constraints:
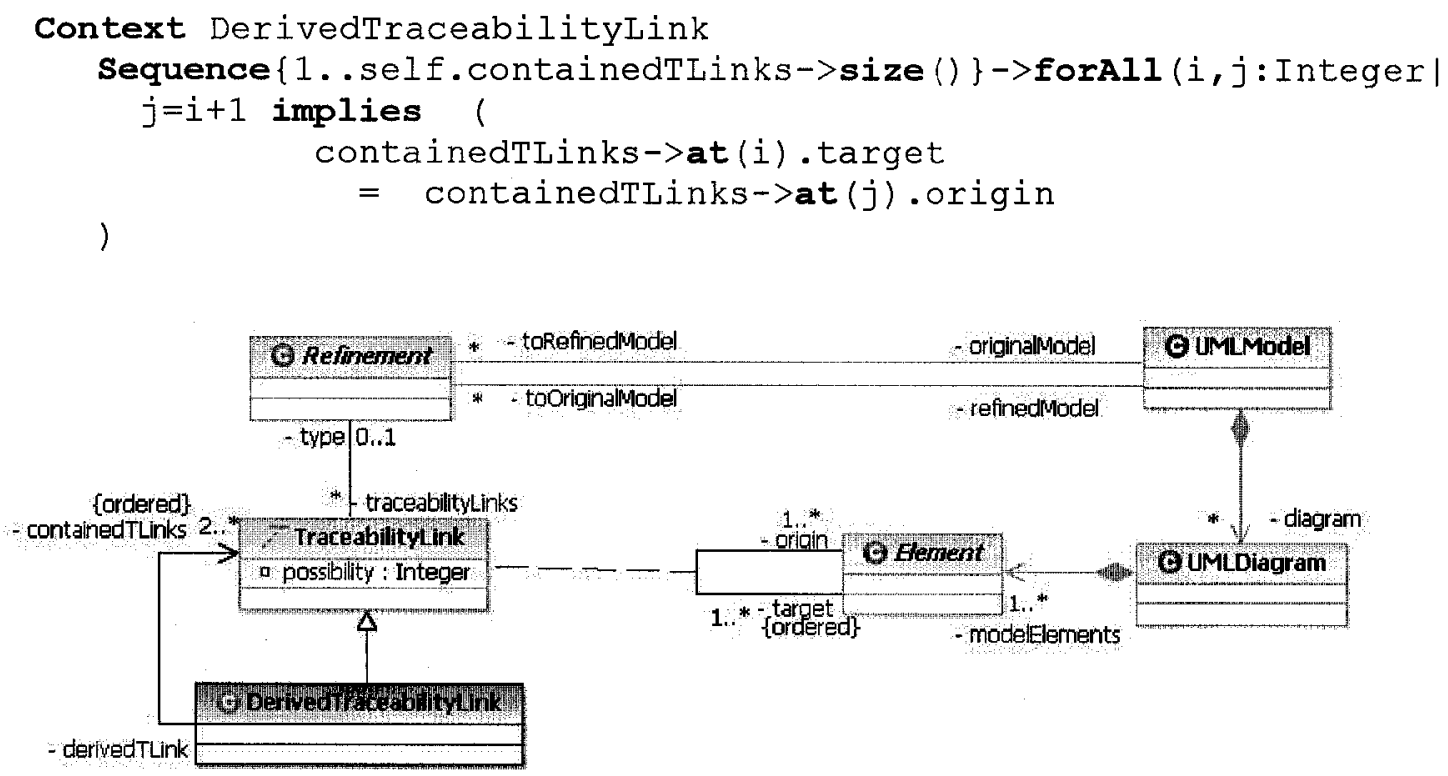

Figure 13 The metamodel for traceability link

As shown in Figure 8, we rely on a group of atomic changes to identify a refinement. Corresponding traceability links are established for this refinement when it is identified. The identification of the refinement will be triggerred when the user executes a command, e.g., saving the model after he/she finished the modifications. 


\subsubsection{Metamodel for performing vertical impact analysis}

Once refinements between two model versions (say, the analysis original model and the design refined model) have been identified and the corresponding traceability links have been established, one can perform vertical impact analysis: class verticalimpact in Figure 14. A verticalimpact relies on the traceability links to identify the elements of the refined model (i.e., impactedElement) that may need to be changed because of a set of changes in the original model (i.e., changedElements). Since traceability links indicate how each original model element is refined, the changes to the original model are necessarily at the origin of some traceability links. Similarly, the set of elements impacted by the Verticalimpact is a subset of the targets of the traceability links. In other words, in the context of class Verticalimpact, we can write the following OCL constraints:

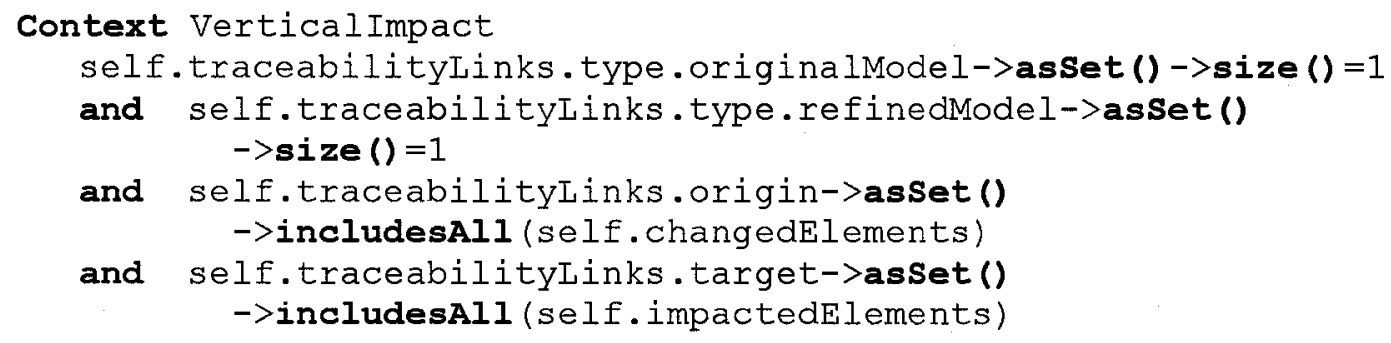

The changed elements in the original model can be the result of a HIA. Similarly, the elements of the refined model being impacted by the VIA can be the starting point of a HIA: modified elements in the original model impact elements of the refined models (VIA) which themselves may impact other elements of the refined model (HIA). This is modeled by the reflexive association connecting the class Impact to itself (i.e., HIAimpacts). HIA is modeled by classes Change and Horizontalimpact, and their associations, which we reuse from an earlier work on UML-based impact analysis [4]. We introduce two classes Vertical Impact and Horizontal Impact as the subclasses of Impact in order to distinguish the impacts caused by performing HIA from the impacts caused by performing VIA. 


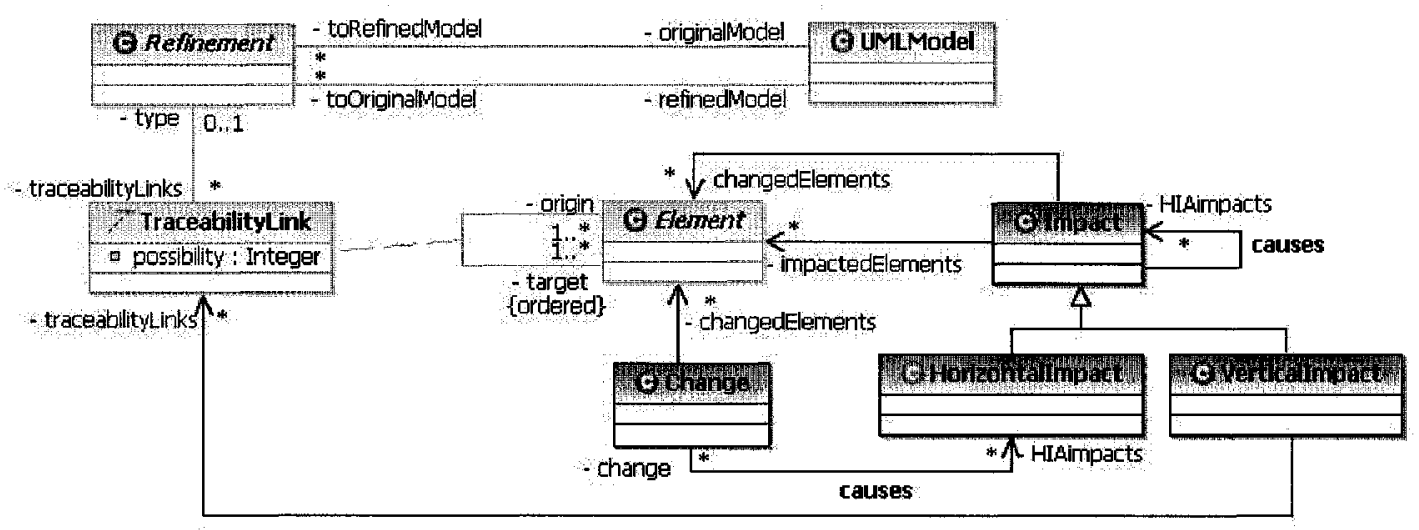

Figure 14 The metamodel for performing vertical impact analysis

\subsection{Taxonomy of Atomic changes}

In order to precisely specify (atomic) refinements we need to precisely specify the atomic changes they can be composed of first. Such a taxonomy already has been presented in [4], where each model element is defined by a set of properties (e.g., a class has attributes) among which core properties uniquely identify the element (e.g., the class name).

We adapted this taxonomy to account for the fact that we rely on the compare\&merge facility of RSA to provide such atomic changes. There are two main reasons for this adaptation. First, in [4], changing the core properties of an element leads to the deletion of the element and the addition of a new element. On the other hand, using RSA, such a situation is classified as a change, i.e., as an atomic change of type changed. Second, moving an element from a location to another (e.g., an operation from a class to another) is classified as a deletion and an addition in [4], whereas in RSA we have the notion of moved element. In other words, we benefit from the precise, fine-grained identification of changes provided by RSA.

Though an Atomicchange can occur in any UML diagram, we focus in this thesis on class diagrams. Figure 15 shows an excerpt of the taxonomy: an AtomicChange in a class diagram can be the addition of a class, the deletion of an association, the move of an attribute between two classes, or the change of a class, which is a change of name or a 
change of property IsAbstract [27], etc. The complete taxonomy for class diagram atomic changes currently contains 47 concrete changes, i.e., leaf nodes in the inheritance hierarchy rooted in ClassDiagramAtomicchange, and can be refined over time if necessary. It is presented in Appendix D.

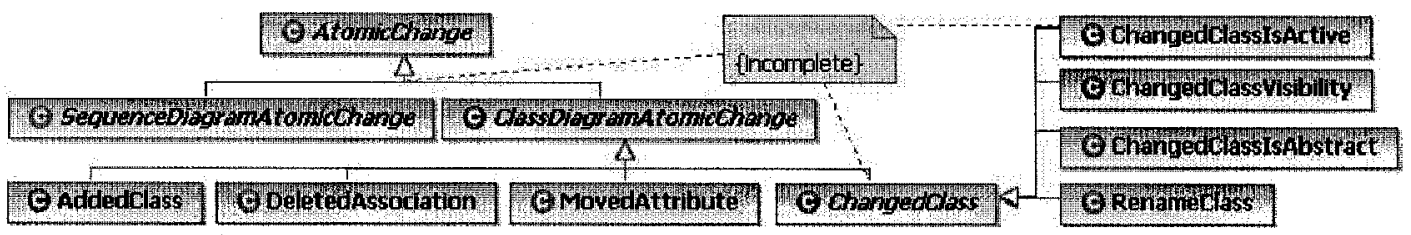

Figure 15 Taxonomy of Atomic Changes

\subsection{Taxonomy of Refinements}

As shown in Figure 16, we also define a taxonomy of atomic refinements, again focusing on class diagram refinements in this thesis. Notice that using the composite design pattern we model the possibility that a refinement involves both class and sequence diagram atomic refinements. (Other diagrams could be added to Figure 16 using the same principle.) We defined our taxonomy of class diagram atomic refinements based on a careful and systematic study of the related literature (conference and journal articles, and text books such as [20]) or according to our own experience of UML-based software development. This taxonomy is also expected to be refined over time.

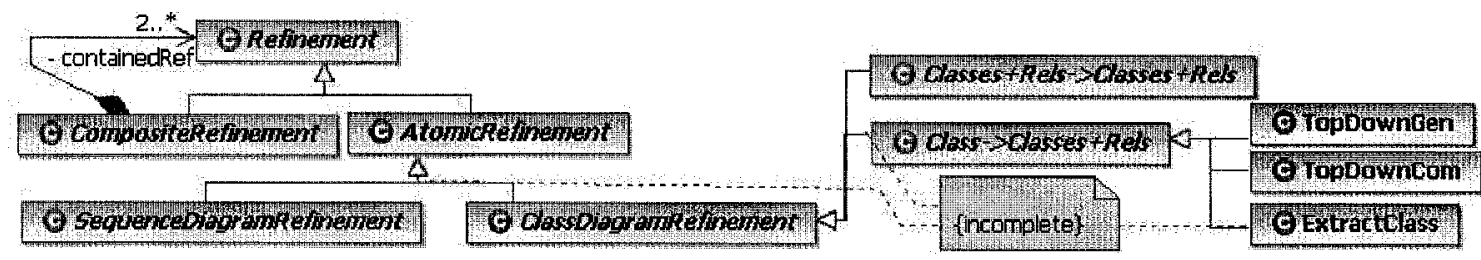

Figure 16 Taxonomy of Refinements

Figure 16 only shows an excerpt of the taxonomy which contains a total of 30 concrete class diagram atomic refinements, i.e., leaf nodes in the inheritance hierarchy rooted in ClassDiagramRefinement. As an example, Class->ClassestRels refers to a family of refinements where a class is refined into a set of classes and their relationships. For instance, it is further specialized in the taxonomy into TopDownGen which corresponds to a refinement through generalization: the class being refined in added a subclass in the 
refined model. Other refinements, not shown in Figure 16, describe for instance the refinement of a set of classes and their relationships into a single class or the refinement of a class into several classes (e.g., splitting of responsibilities). The complete taxonomy for class diagram refinements is presented in Appendix E.

\subsection{Refinement/Traceability link specification}

As indicated in Figure 8, an atomic refinement may be derived from one or more atomic changes. We distinguish them as they are formalized in different ways. These two types of refinements and their formalizations are discussed in Section 4.4.1 and 4.4.2, respectively.

\subsubsection{Atomic refinements derived from a single atomic change}

This is the simplest one: once such an atomic change has been detected, an atomic refinement, and corresponding traceability links can be detected automatically. As we discussed in Section 4.2 we rely on the compare\&merge engine (Section 5.2.1) of RSA to detect atomic changes and as a result atomic refinements can be detected automatically. Each of this type of atomic refinements leads to creating a traceability link except the atomic refinements derived from a single added or deleted atomic change. The strategy for establishing traceability links is as follows.

\section{Changed:}

For the atomic refinement derived from a single atomic change of type changed, a traceability link between the original model element in the original model (i.e., the affectedelement of the atomic change) and the refined model element in the refined model should be established.

For example, a traceability link is specified in Figure 17 (b) for a Class IsAbstractRef refinement, which is detected from a single atomic change of type ChangedClassIsAbstract: the class is changed into an abstract class (specified in Figure 17 (a)). The affected element of such a change is a class. The refinement's unique traceability link is between the original class (i.e., the affectedElement of the 
atomic change) and the refined class. We assume that the original model is refined into the refined model by the ClassisAbstractRef refinement only. We can identify the refined class (referred to as refclass) by looking for the class in the refined model which has the same name as the original class (i.e., the affected element of the atomic change ChangedClass IsAbstract) (referred to as origClass).

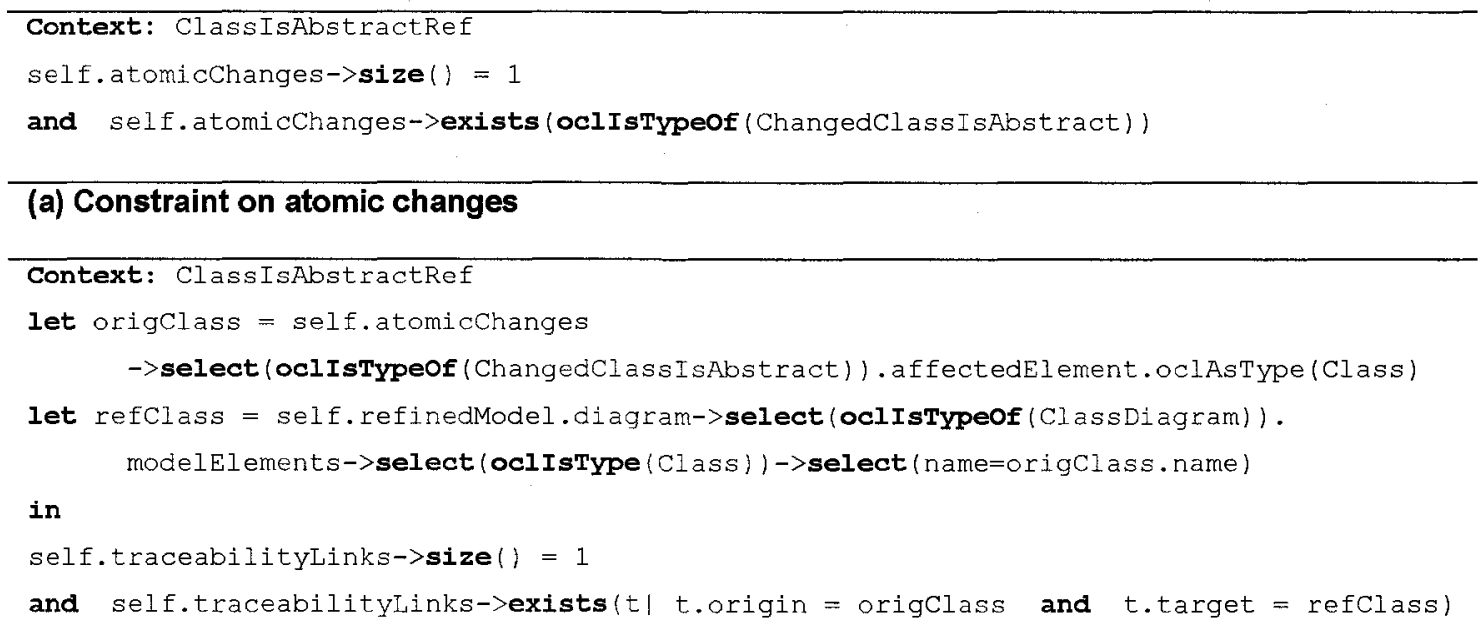

Figure 17 Refinement Class IsAbstractRef

\section{$\underline{\text { Moved: }}$}

For the atomic refinement derived from a single atomic change of type moved, a traceability link between the original model element in the original model (i.e., the affectedElement of the atomic change) and the refined model element in the refined model (i.e., the model element moved to the diagramLocation or targetLocation) is established.

For example, a traceability link is specified in Figure 18 (b) for a MovedAttributeRef refinement, which is detected from a single atomic change of type MovedAttribute: the attribute is moved from a class into another (specified in Figure 18 (a)). The affectedElement of such a change is a Property. The traceability link is between the attribute in the original class (i.e., the affectedelement of the atomic change) and the attribute in the new location (i.e., the attribute in the targetLocation of the atomic change). 


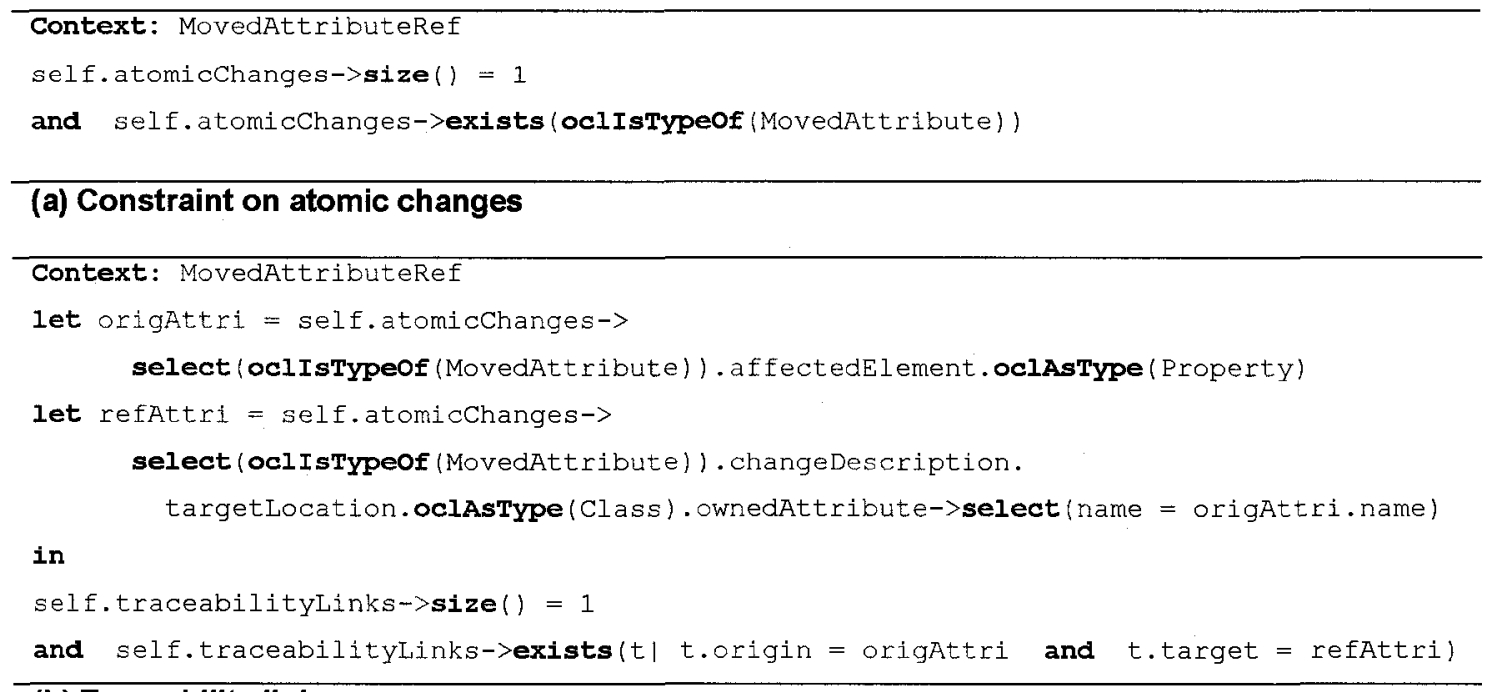

(b) Traceability links

Figure 18 Refinement MovedAttributeRef

\section{Added or Deleted:}

No traceability link should be established for the atomic refinements derived from a single Added atomic change, since there is no model element in the original model which can be linked to.

Similarly, no traceability link should be established for the atomic refinements derived from a single Deleted atomic change, since there is no model element in the refined model which can be linked from.

\subsubsection{Atomic refinements derived from several atomic changes}

Atomic refinements derived from several atomic changes are more complex to handle. Therefore, a systematic strategy for formalizing this kind of atomic refinements is required, and is introduced in this section. The specification and formalization of all concrete class diagram atomic refinements that appear in the taxonomy of Section 4.3 are presented in Appendix E. Some of them are exemplified in the case study section (Section 6).

Each concrete refinement in the taxonomy is rigorously specified, following a template description containing: a general description of the refinement; a description of the user's 
intent; the list of atomic changes that must be present, and the constraints they must satisfy to identify such a refinement; and a description of the corresponding traceability links. Constraints on atomic changes and traceability links are described using the OCL. We believe that, if we want such research to converge over time and other researchers to build on it, the method employed for detecting changes, refinements and establishing traceability links needs to be rigorously specified.

When a type of atomic refinement is specified, we assume that the original model is refined into the refined model by this type of refinement only. It means that no other refinements are performed before or after this refinement on the original model. This assumption applies to all specifications of atomic refinements. Of course, there is the case in which several independent refinements need to be identified together and corresponding traceability links need to be established. There exists an order in which these refinements should be identified. Finding such an order is however part of our future work and we currently rely on a multi-pass heuristic: OCL rules identifying refinements are all evaluated once, twice, and so on, until no new traceability link is established.

As an example, consider the class diagram atomic refinement Class $->C l a s s e s+R e l s$ (Figure 16) which refers to a family of refinements where a class is refined into a set of classes and relationships. One example of such a refinement, is called TopDownGen (Figure 16): the class is added a subclass (a class is refined by means of a top-down generalization).

The specification of refinement TopDownGen, i.e., the characterization of its atomic changes and their relations, is shown as the OCL invariant for class TopDownGen in Figure 19 (a). The expression indicates that a TopDownGen refinement is composed of two atomic changes of type AddedClass and AddedGeneralization (first three terms in the conjunction). Additionally, the newly added generalization relationship connects the newly added class (as the subclass) to the class being refined (last conjunct).

For such a refinement, two traceability links have to be established: one between the original class and the superclass of the added generalization; one between the original 
class and the added subclass. The rationale is that if a change to the original class (in the original model) is performed, or if this class is impacted by some horizontal impact analysis, then both the super class and the child class of the added generalization (in the refined model) may need to be updated.

These traceability links are specified in the OCL expression of Figure 19 (b). The first four let expressions define local variables for the rest of the constraint: newGen refers to the newly added generalization; superclass and subclass refer to the parent and child classes of the added generalization, respectively; origclass refers to the class in the original model that is being refined which is identified by looking for the class in the original model which has the same name as the superclass. Using these local variables, the rest of the expression (after the in keyword) indicates that there are two traceability links for the TopDownGen refinement, one between origClass and superClass, and one between origclass and subclass.

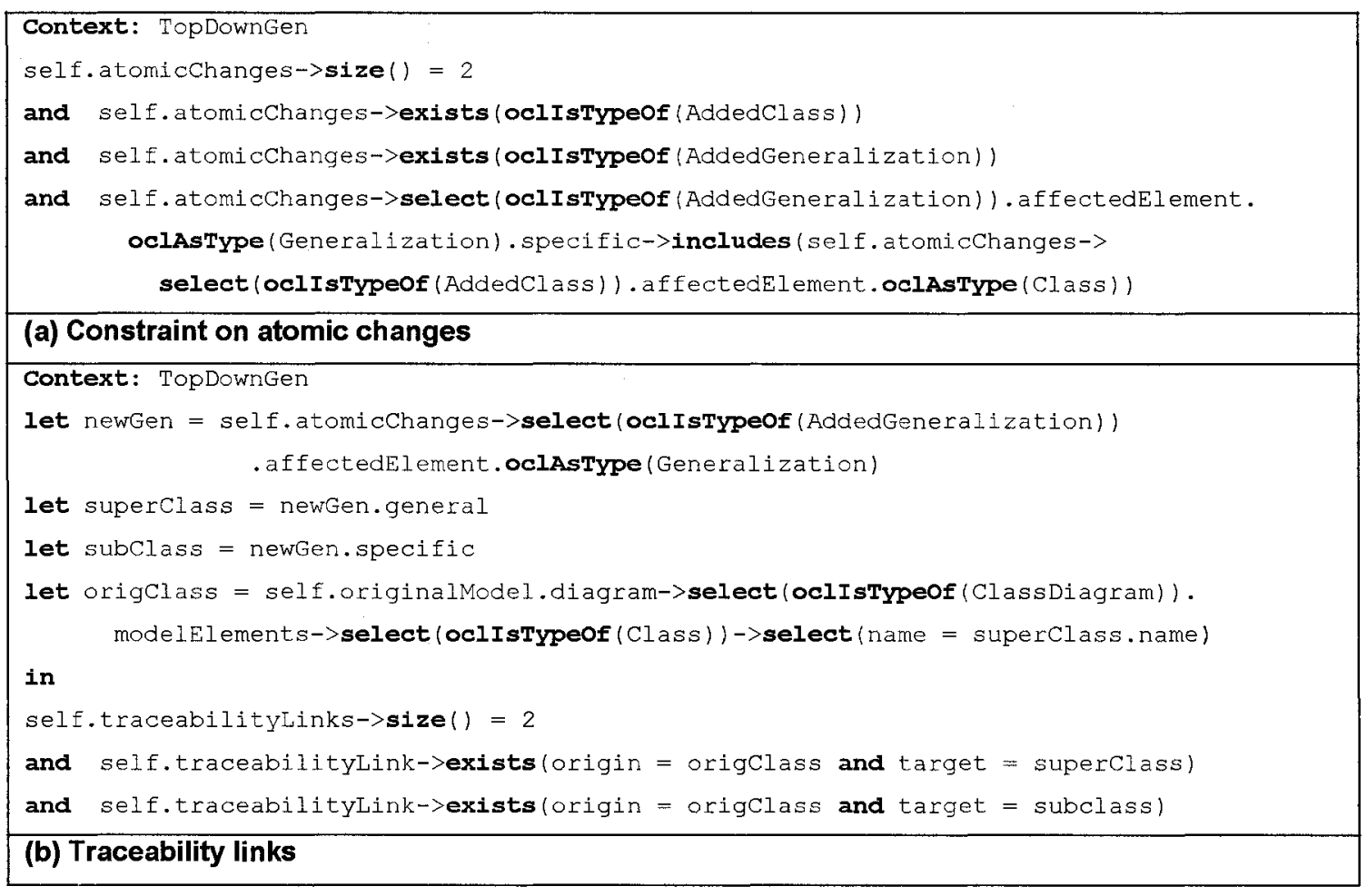

Figure 19 Refinement TopDownGen 


\section{AUTOMATION}

Our approach is implemented in a tool named VIATOOI (Vertical Impact Analysis Tool). The tool is composed of two subsystems as illustrated in Figure 20: VIATraceability and VIAPerform. Subsystem VIATraceability identifies refinements and establishes traceability links between two UML models. The VIAPerform subsystem performs vertical impact analysis with the traceability links as input.

In the VIATraceability subsystem, the VIA Eclipse Plugin is a plugin to the Eclipse platform that interacts with two other Eclipse plugins, namely Eclipse UML2 [11] and Eclipse EMF, which will be further discussed in Section 5.1.1. The Eclipse UML 2 project is an EMF-based implementation of the UML 2.0 metamodel [27] for the Eclipse platform, and is integrated into IBM's Rational Software Architect (RSA) [16].

VIA Eclipse Plugin also interacts with the VIA Capturing Traceability Engine which is responsible for identifying refinements and establishing traceability links. The VIA Capturing Traceability Engine obtains a group of atomic changes from the CompareMerge Engine, which is part of RSA, for identifying atomic changes [16]. The AtomicChange Model is an EMF-based implementation of the atomic change concepts discussed in Section 4.1.3. The model will be instantiated according to the deltas obtained from the CompareMerge Engine, then it will be used by the VIA Capturing Traceability Engine to identify refinements. The VIA Capturing Traceability Engine identifies a refinement by checking refinements in the Refinement Repository against the group of atomic changes and UML models. Last it adds new traceability links to the Traceability Repository. The Refinement Repository is used to model refinements. The Traceability Repository is used to model traceability links. They are both based on EMF. 


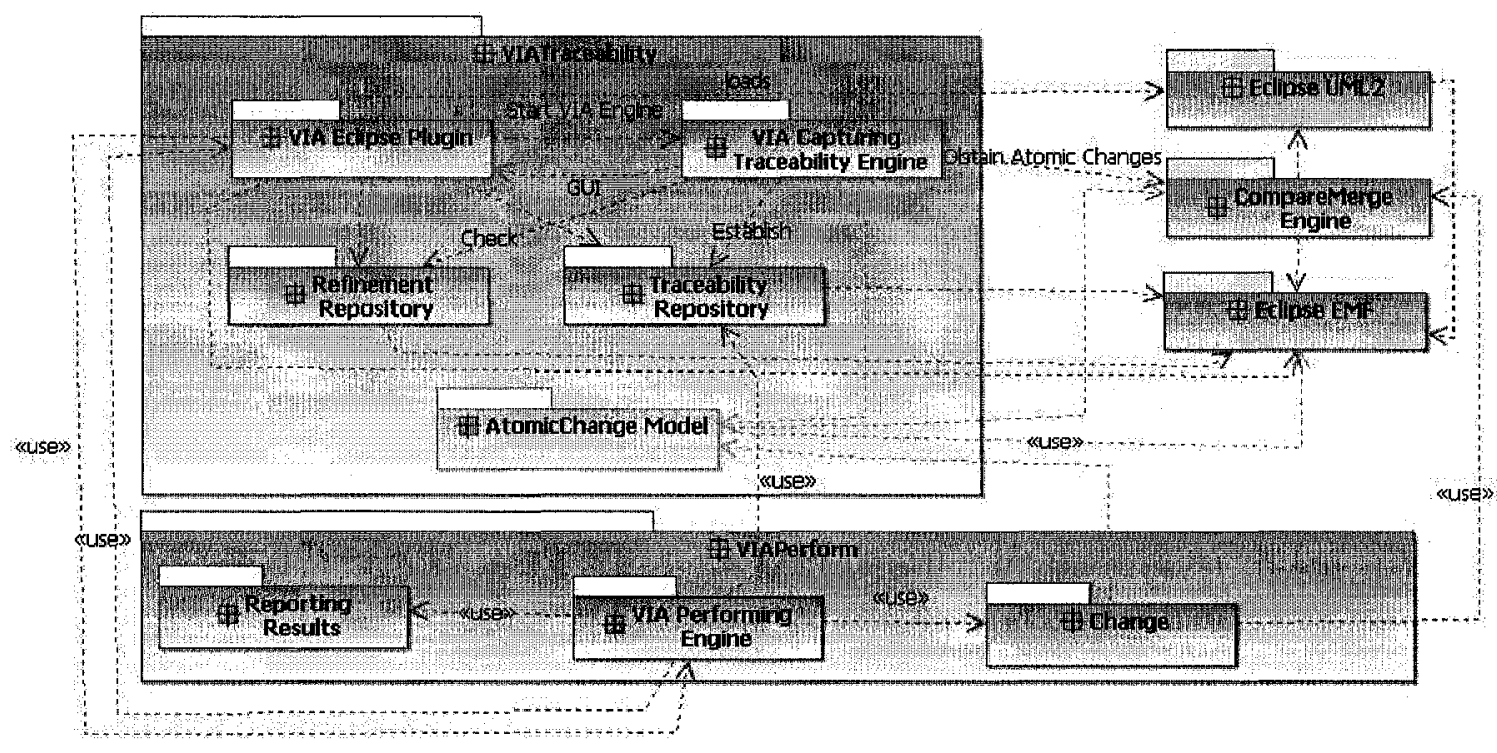

Figure 20 VIATool Architecture Overview

In the VIAPerform subsystem, the VIA Performing Engine interacts with the VIA Eclipse Plugin to obtain the UML models to be analyzed. Vertical impact analysis is triggered by a change (the Change subsystem), which is obtained using the CompareMerge Engine. The VIA Performing Engine performs vertical impact analysis with two UML models, a change, and traceability links (stored in the Traceability Repository) as inputs. Then a report is generated, Reporting Results to show the analysis results. The UML models to be analyzed are also instantiated using the Eclipse UML2 project.

The following sections further detail the design of the system. We start with a short description of the Eclipse platform and its related projects.

\subsection{The Eclipse Platform}

Eclipse is an open source, robust, full-featured, industry platform for the development of highly integrated tools and applications. The plug-in based framework makes it easier to create, integrate and utilize software tools. The objective of the Eclipse project is to become an industry platform and to meet the needs of the Eclipse tool building community. 
The Eclispe project is composed of itself many subprojects or categories, e.g., The Eclipse Project, The Eclipse Tools Project [9], The Eclipse Technology Project [8]. The two categories of interest for this thesis are the Eclipse Project and the Eclipse Tools Project. The Eclipse Project contains both the Eclipse platform technology, and a set of tools that together form the software development kit (SDK) for building Eclipse-based tools: The Java Development Tooling, or Java IDE [6] and the Plug-in Development Environment [10]. These tools are used to develop the initial prototype of the VIATool and are used in the execution of the tool. The Eclipse Tools Project contains several smaller projects not essential to the Eclipse Platform, but can be seen as value added features. Two of these projects are critical in the implementation of the VIATool, namely the Eclipse Modeling Framework (Section 5.1.1) and the UML2 Project ([11]).

The Eclipse platform provides the foundation from which many commercial tools have been developed (e.g., IBM RSA). VIATool also makes use of the OCL engine (Section 5.1.2) which is a component of the IBM RSA.

\subsubsection{Eclipse Modeling Framework (EMF)}

The Eclipse Modeling Framework (EMF) is a modeling and code generation engine for building applications based on a structured data model. Using EMF, a developer can define a data model and get a set of Java classes, automatically generated by the EMF facility, to create and manipulate instances of the data model. EMF also produces a set of adapter classes to enable viewing and editing the model, and a basic editor, which is itself an Eclipse plugin [7]. The data model can be specified using annotated Java, XML documents, modeling tools like Rational Rose, or directly use the EMF editor which is provided along with RSA. We use this EMF editor to build VIATool models which will be discussed in Section 5.3. From a model definition, EMF can generate Java implementation code including UI, XML Schemas, Eclipse projects, and plug-ins. A typical EMF usage scenario could be:

1. Create an Ecore model. You can choose to import it from UML (e.g., Rational Rose with Ecore profile, .mdl file), an XML Schema, or an Ecore model directly using EMF's Ecore editor; 
2. Generate a generator model which provides access to all of the data needed for code generation. Besides, it is automatically kept in synch with the Ecore model. Code generation options can be specified at this time as well;

3. Generate Model, Edit, Editor and Test code separately or generate all of them together. Each set of code is an Eclipse plug-in. EMF functionality for data persistence is added when the EMF java code is generated;

4. Instantiate the model with instance data using the generated EMF model editor. These instances can be serialized into XML and deserialized from XML as well;

5. Iteratively refine the model (and regenerate code) and develop the corresponding java application;

6. Optionally, use the generated .Edit to build customized user interface.

The EMF technology is used in our VIATool to build the Refinement Repository, Traceability Repository and AtomicChange Model which will be further discussed in Section 5.3.

\subsubsection{The OCL Engine}

Along with EMF, IBM has defined an OCL Engine which has been included in the IBM RSA version 6. The OCL Engine can be used to evaluate an OCL statement (i.e., String object) to query the characteristics of your metamodel. For example, you can evaluate an OCL statement that returns a simple type, such as a Boolean value, or you can evaluate an OCL statement that returns a list of metamodel objects.

In VIATool, the OCL engine is used to evaluate and query constraints for identifying refinements and establishing traceability links. These constraints are specified using OCL expressions and provided along with the instances of the Refinement Repository model. 


\subsection{Rational Software Architect}

IBM RSA is an integrated design and development tool that leverages model-driven development with the UML for creating well-architected applications and services. RSA unifies all aspects of software design and development into one powerful and easy to use tool. It supports understanding, designing, managing and evolving enterprise solutions and services.

RSA leverages the Eclipse open source APIs, including EMF and the UML 2 metamodels. It supports the major UML 2 diagrams. OCL is supported for specifying constraints as well (Section 5.1.2).

Since IBM RSA is built upon the Eclipse integrated development envinronment, it inherits Eclipse's compare support work flows. IBM RSA is itself built using the EMF, which essentially means that it contain complex structured data and cannot successfully be understood as text. Therefore, a custom EMF and UML compare support has been implemented, namely the compare\&merge engine (Section 5.2.1).

RSA is built on the top of the open and extensible Eclipse platform and it provides an open and extensible modeling platform. We choose RSA as the platform for our VIATool's development due to the following reasons:

1. RSA supports graphically editing and querying UML 2 models;

2. RSA has compare support which is used to obtain atomic changes in our VIATool;

3. RSA supports the evaluation of OCL expressions which facilitates greately the implementation of our VIATool.

\subsubsection{Compare\&merge engine}

The compare\&merge engine is developed by IBM and it is used in two common scenarios: 
- Compare with local history, which is useful for looking at the evolution of a model over time. Local history is a check-point where each saved version is retained for an unspecified period of time. This is the scenario we are using in the VIATool.

- Compare with each other, which allows for comparison between two or three versions of a model in your workspace.

The compare\&merge engine, as its name indicates, also supports merging two versions of UML models. Since this is not the case that we are interested in, we will not discuss this functionality any further.

\subsection{EMF Models in VIATool}

The VIATool contains three EMF models: Refinement Repository, Traceability Repository and AtomicChange Model. These models were described in Section 4.1.1, Section 4.1.4, and Section 4.1.3 respectively. Instantiating these models is automated using EMF. As mentioned in Section 5.1.1, EMF can generate a basic editor for the models generated by EMF. The EMF editor is an Eclipse plugin and once integrated into Eclipse the EMF models can be generated under existing projects (Figure 21).

We use the Refinement Repository model here to exemplify how EMF models work. The use of the editor of the Refinement Repository model will be illustrated using an example: the TopDownGen refinement discussed in Section 4.4.2. The model instance is created with the AtomicRefinement (i.e., Atomic Ref in Figure 22) object as the root of the tree. The Properties view allows the developer to fill the attributes of the object.

Then the instance of the Refinement Repository model is populated by the atomic changes required to identify it, the traceability links which should be established for the refinement, and the user's help if required (Figure 23). Once all the required objects for the model are created the information for each object can be edited in the properties view (Figure 24). 


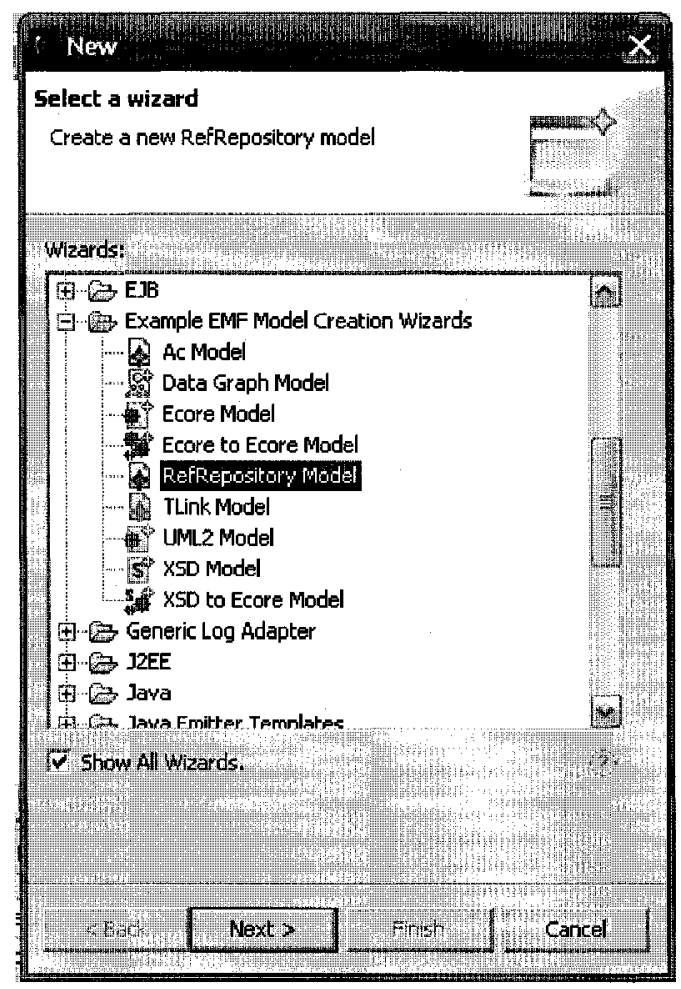

Figure 21 Creating a new refinement repository model

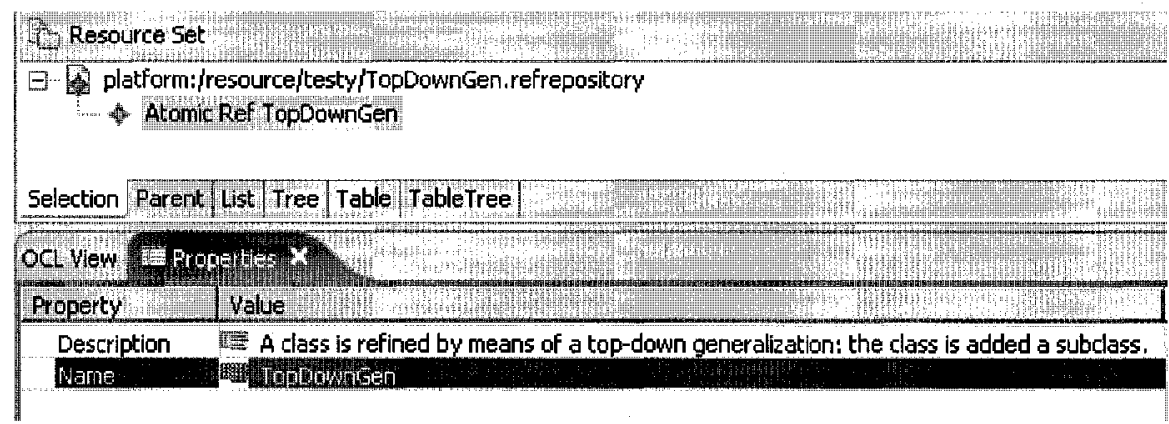

Figure 22 Creating a TopDownGen refinement object 


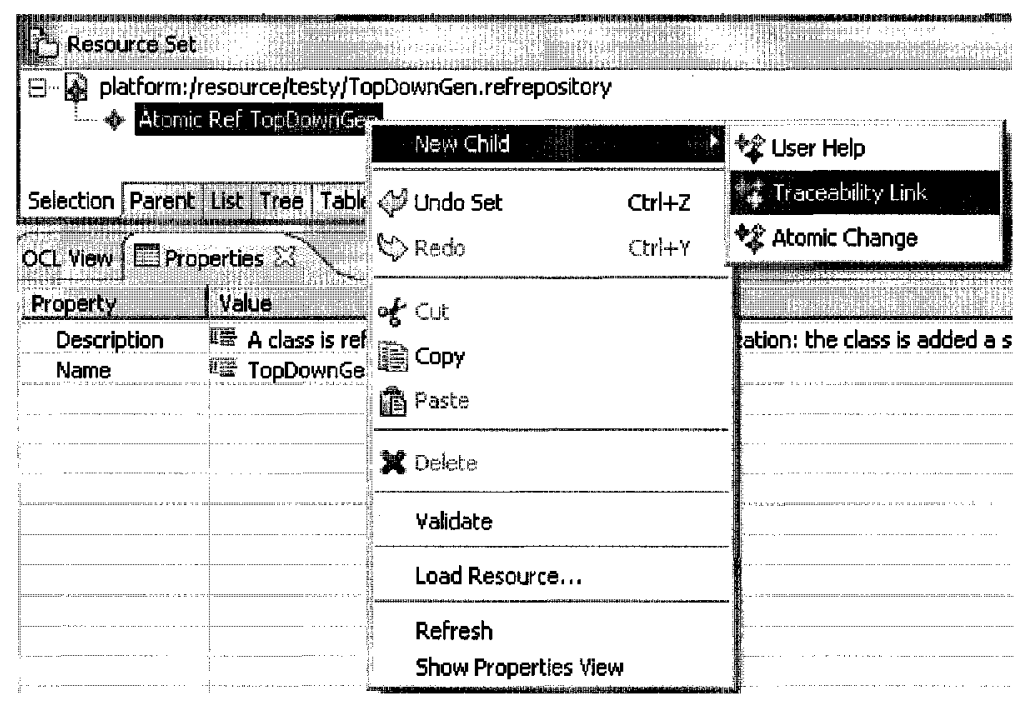

Figure 23 Creating new objects using the editor

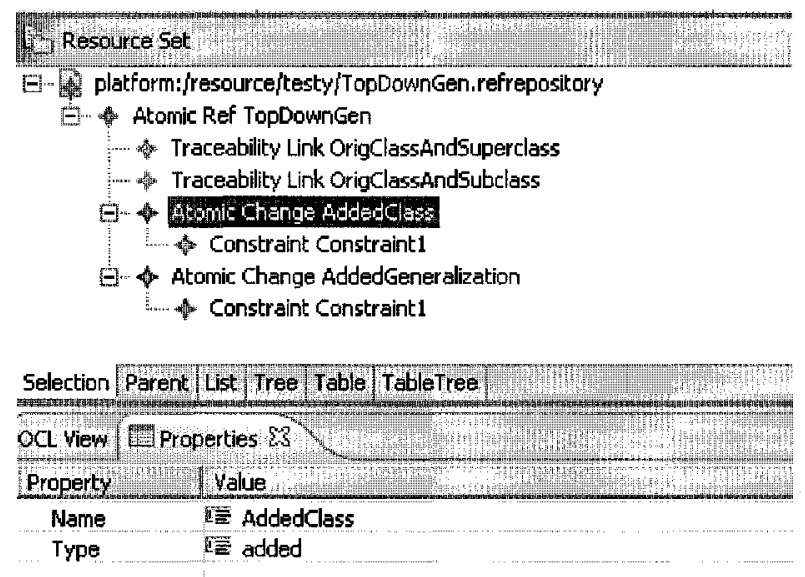

Figure 24 Editing Attributes and Associations using the editor 


\section{CASE STUDY}

Figure 25 is a case study we selected from [20]. Figure 25 (a) shows the analysis model, whereas Figure 25 (b) shows the design model. The analysis model has been transformed into the design model by applying 12 refinements, from a set of seven refinement types. The changes made to the design model are the following: Class Payment has been transformed into an abstract class and new subclasses (e.g., CashPayment); The association between Payment and sale has been transformed into new classes (e.g., Customer) and associations; Attribute price in class ProductDescription has been turned into a class, namely ProductPrice; A number of associations have been updated: change of navigability (e.g., between store and sale), change of multiplicity (e.g., between ProductDescription and SalesLineItem), change of association type (e.g., between sale and SalesLineItem). These changes correspond to the following atomic changes in our taxonomy: ChangedClassisAbstract, AddedClass, AddedGeneralization, DeletedAssociation, Added-Association, DeletedAttribute, ChangedAssoEnoMultiplicity, ChangedAssoEndIsNavigable, and ChangedAssoEndType.

These atomic changes are used to identify 12 refinements, corresponding to seven refinements types, namely: TopDownGen, ClassisAbstractRef, DetailAssoFunctionality, TurnAttriIntoclass, AssoEndMultiplicityRef, AssoEndIsNavigableRef, and AssoEndTypeRef. We have already described the specification of the first two of those refinements in Section 4.4, along with their rules to establish traceability links. In this section, we address the remaining five refinement types. 


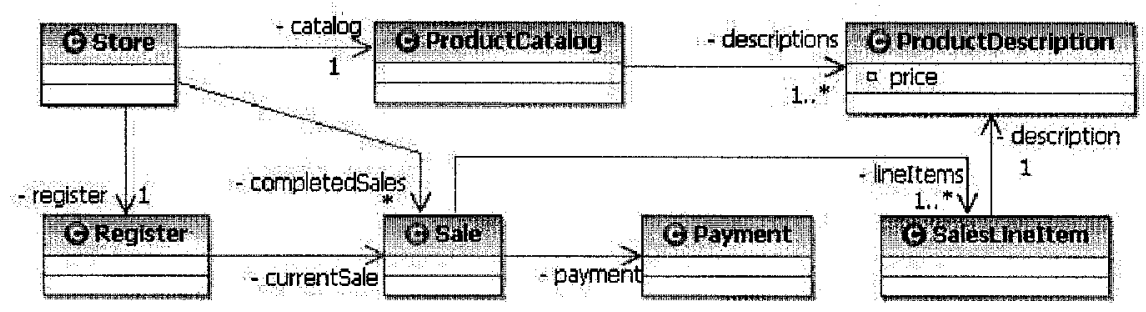

(a) Analysis model

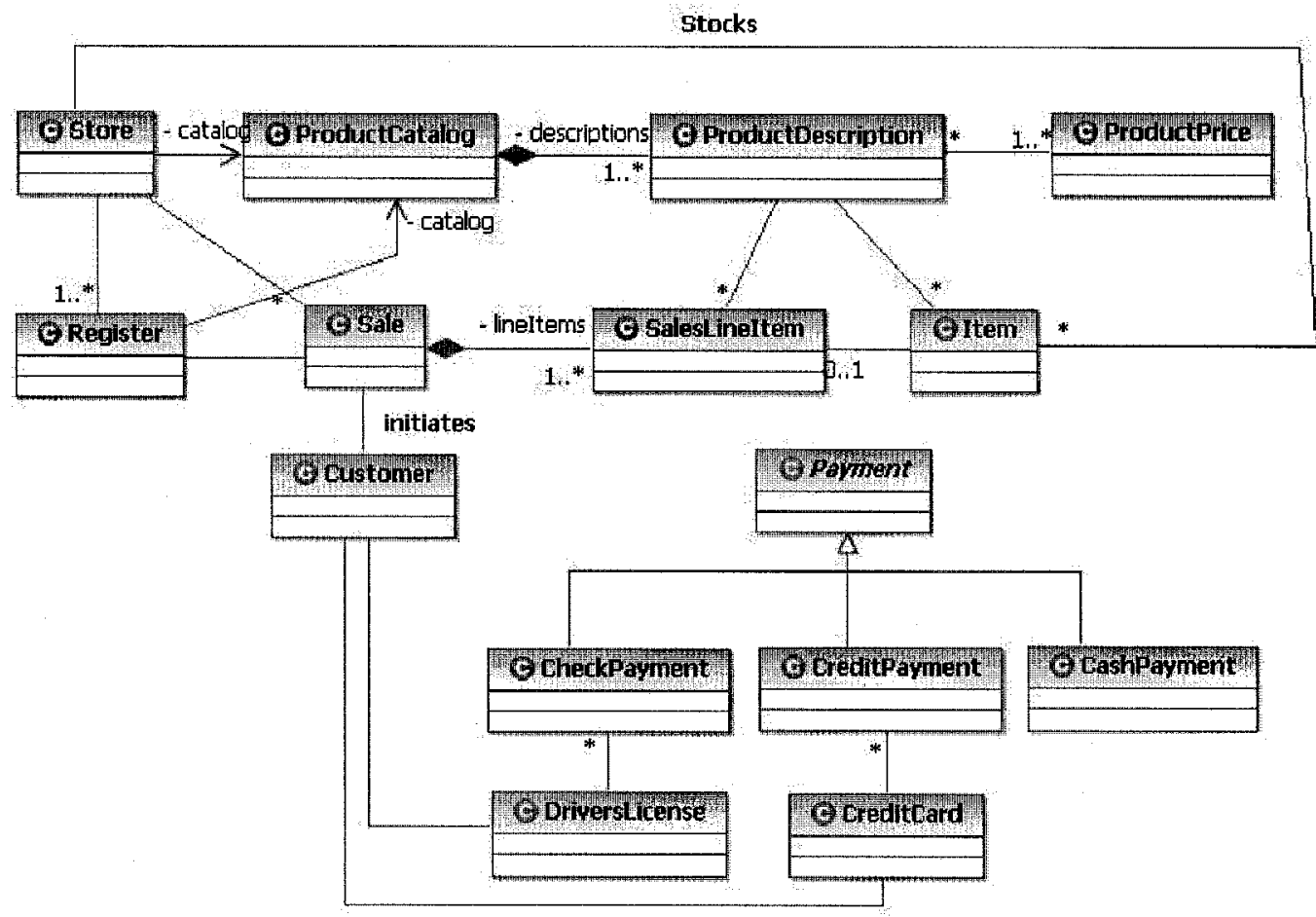

(b) Design model

Figure 25 Case Study (from [20])

\subsection{DetailAssoFunctionality}

Figure 26 shows the specification of refinement DetailAssoFunctionality, whose purpose is to refine an association by adding new classes and associations. The general idea for identifying such a refinement is to be able to find, in the refined class diagram, at 
least one path between the classes at the two ends of the association ${ }^{6}$ being deleted in the original model. Referring to the example of Figure 25 (b), we are interested in paths between abstract class Payment (and its three subclasses) and class sale. We need to rely on already identified refinements here, i.e., the refinements of the two ends of the deleted association.

As specified in Figure 26 (a), such a refinement is at least made of one DeletedAssociation atomic change: the deleted association (in the original model) is referred to as deletedAsso. The next let expressions are used to identify the two ends of the deleted association in the original model (origA and origB) and how they may have been refined (refA and $\operatorname{ref} B$ ). Last, we use operation exists () of class Path to identify whether there is a path between refA and refB in the refined model. Class Path does not appear in our metamodel as it is only a helper class used to shorten our OCL expressions. Its formalization is given in Appendix F. A Path instance is associated with a sequence of Elements, i.e., a path is a sequence of UML model elements. Operation exists (endA, endB), endA and endB being classes of the same model, returns true if and only if there exists a path between these two classes. Operation exists (endSetA, endSetB), where the two parameters are sets of classes, is a similar variant: it returns true if we can find a path between any pair of classes, each class in the pair belonging to one of the two sets. It is this latter form that is used in Figure 26 (a) since refA and refB are sets in this case. In our example, one of the two sets is \{sale\} and the other is \{Payment, CashPayment, CreditPayment, CheckPayment\} (class Payment in the analysis model has been refined using the TopDownGen refinement).

Figure 26 (b) specifies how traceability links are established for refinement DetailAssofunctionality. For each path that we can find in the refined model between refA and refB (we use operation getPaths () of class Path), we establish a traceability link between the removed association origAsso (in the original model) and each element of the path (i.e., associations and classes). In our example, this results in a traceability link between the association between Payment and sale (analysis model)

\footnotetext{
${ }^{6}$ In this article, we assume associations are binary, although n-ary associations can be handled following the same principles. Future work will adapt our OCL expressions to account for n-ary associations.
} 
and CreditPayment, CreditCard, CheckPayment, DriverLicense, Customer, and their relationships, in the design model.
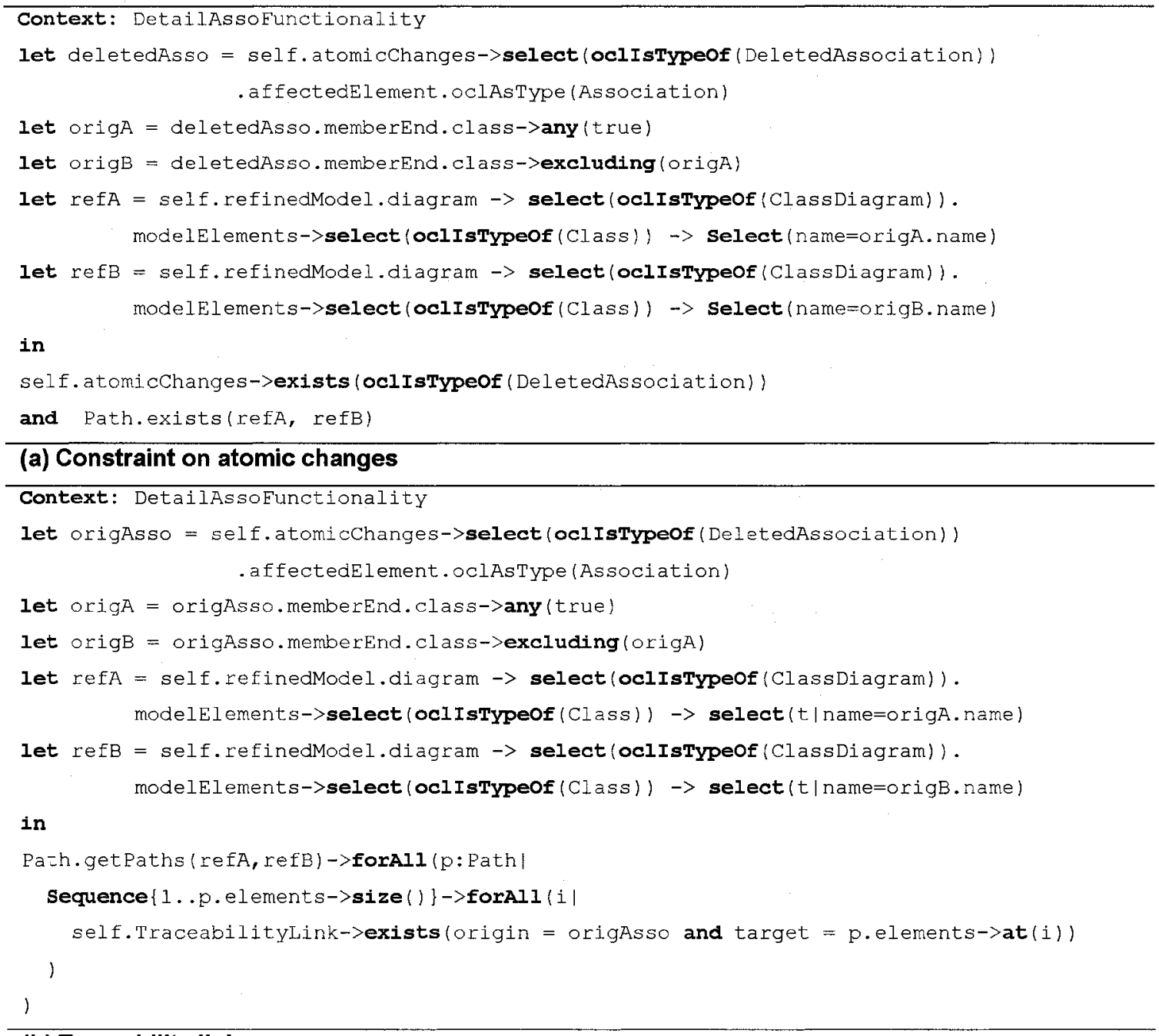

(b) Traceability links

Figure 26 Refinement DetailAssoFunctionality

\subsection{TurnattriIntoclass}

A TurnattriIntoclass refinement means that an attribute of a class has been removed, a class has been added, and an association has been added between the added class and the class from which the attribute has been removed (Figure 27 (a)). The refinement is thus based on three atomic changes of types AddedClass, AddedAssociation and Deletedattribute. In addition, one end of the added association should be the added class, and the other end should be the refinement of the class from which the attribute has 
been removed (this check is the purpose of the last five lines of the expression). Referring to Figure 25, Attribute price in class ProductDescription has been turned into a class, namely ProductPrice according to the TurnAttriIntoclass refinement type.

Once a TurnAttriIntoclass has been detected, three traceability links have to be established (Figure 27 (b)): between the original class (origclass) and the new class (newClass) and association (newAsso), and between the original class and its corresponding refined class (refinedclass) in the refined model. Indeed, we need those three traceability links since if changes are made to the original class (e.g., changing its attributes), we may have to update the refined class, the added association and the added class (e.g., if some attributes are added to the original class, a subset of those may have to be moved to the added class in the refined model).

Note that we do not need to establish similar traceability links between the deleted attribute and the added association and class. Indeed, if a change is made to this attribute in the original model, its class will be "horizontal" impacted. As a result, thanks to the three established traceability links, the added association and class will be "vertical" impacted. 


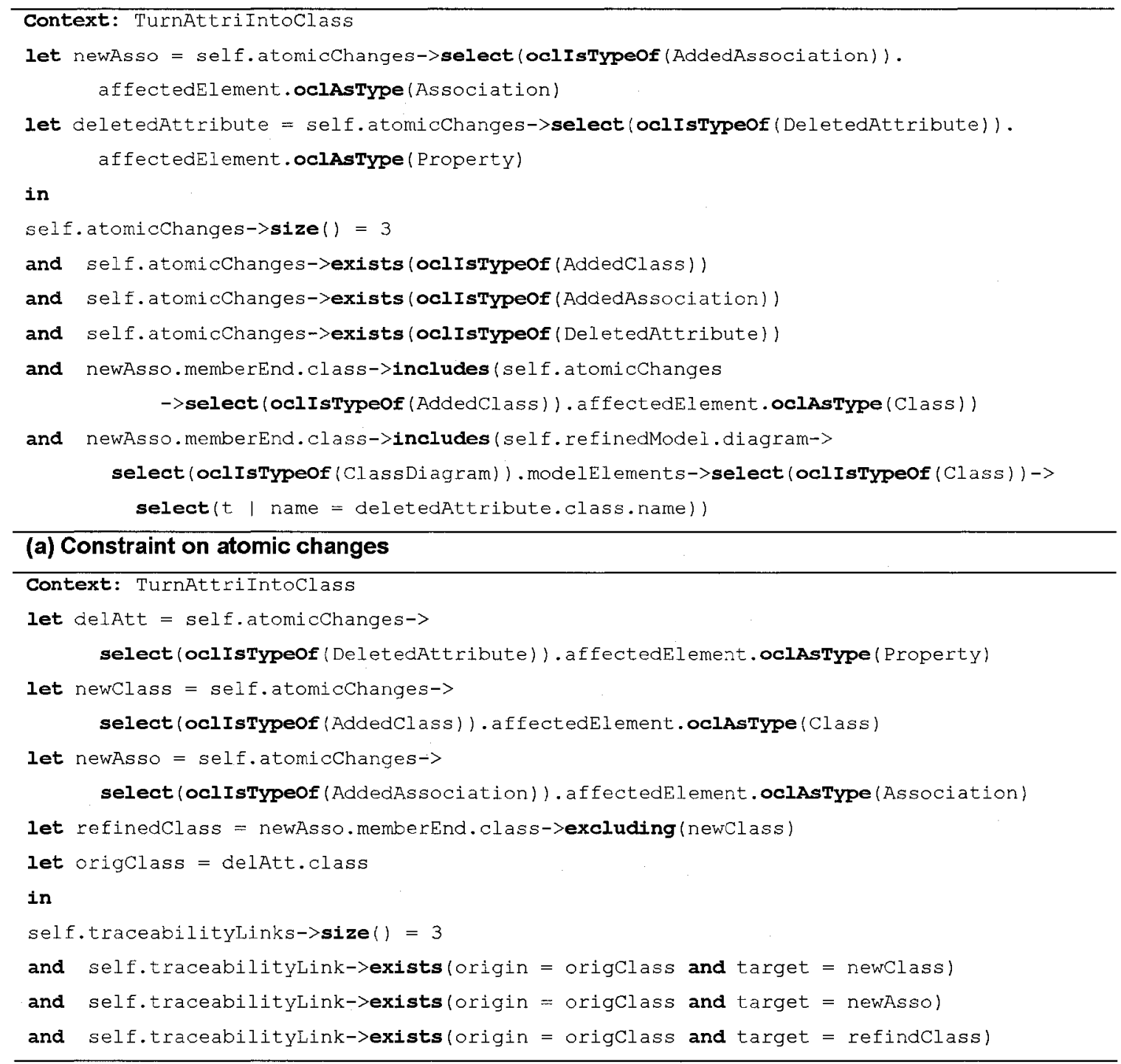

(b) Traceability links

Figure 27 Refinement TurnAttri Intoclass

\subsection{AssoEndMultiplicityRef}

An AssoEndMultiplicityRef refinement is simple as it is detected, as specified in Figure 28 (a), from a single atomic change of type ChangedAssoEndMultiplicity (the affected element of such a change is an Association). Referring to Figure 25, the multiplicity on the class Register end of the association between classes store and Register is refined according to the AssoEndMultiplicityRef refinement type: the original multiplicity of 1 is changed into $1 .{ }^{*}$. A similar refinement has also taken place 
on the class SalestineItem end of the association between class ProductDescription and SalestineItem.

Once such an atomic change has been detected, a refinement, and corresponding traceability links can be specified: Figure 28 (b). The refinement's unique traceability link is between the original association (i.e., the affectedElement of the atomic change) and the refined association, e.g., between the association between classes store and Register in Figure 25 (a) and the association between classes Store and Register in Figure 25 (b). The refined association in the refined model is identified by looking for the association end being refined (referred to as refAssoEnd) in the refined model. The class owning the association end (Property) has the same name as the class owning the original association end (referred to as origAssoEnd), the class owning the other association end of the association has the same name as the class owning the other association end of the original association, and the multiplicity (i.e., upper and/or lower) of the original association end is changed, in order to establish a new traceability link corresponding to the AssoEndMultiplicityRef refinement.

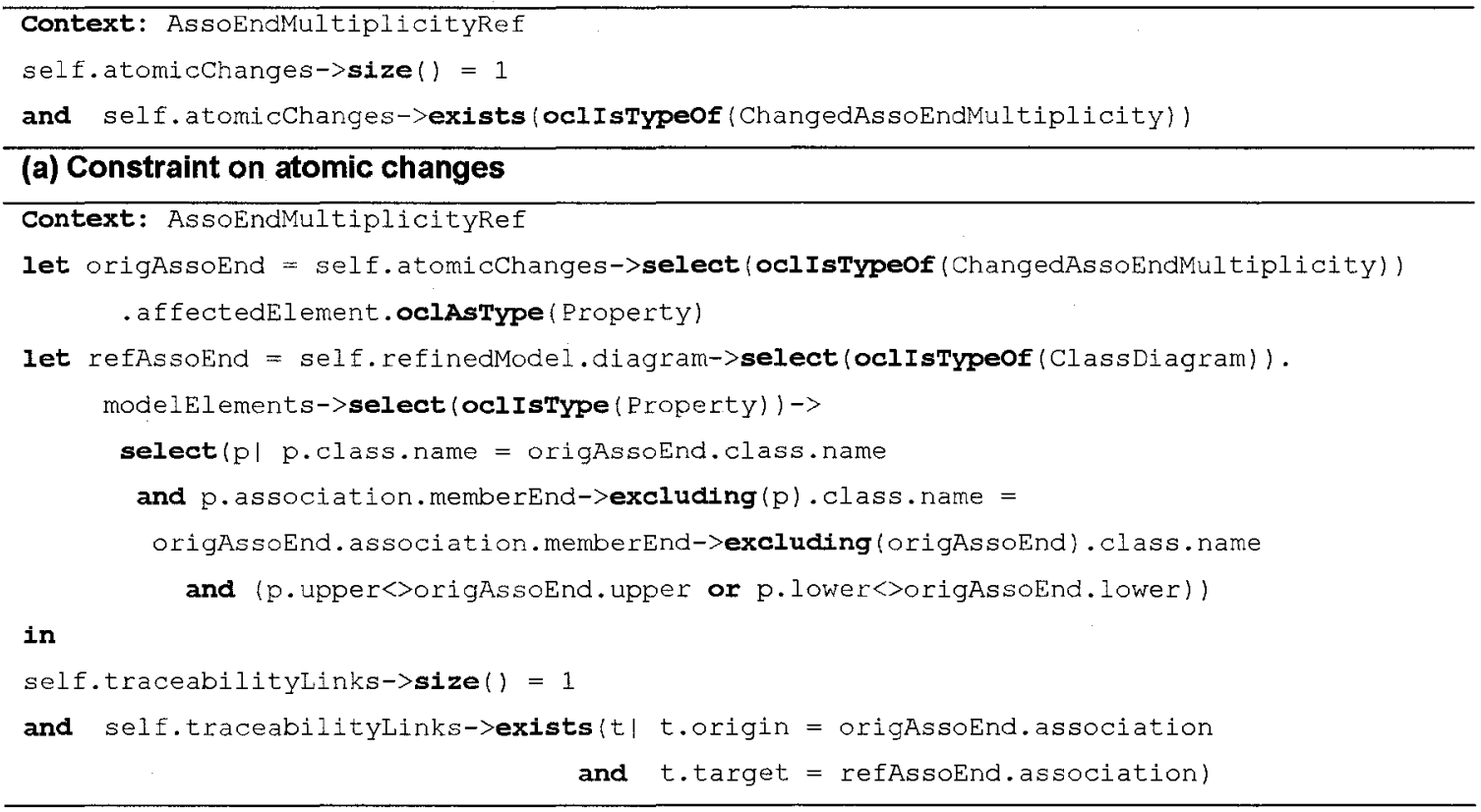

(b) Traceability links

Figure 28 Refinement AssoEndMultiplicityRef 


\subsection{AssoEndIsNavigableRef}

An AssoEndIsNavigableRef refinement is simple as it is detected, as specified in Figure 29 (a), from a single atomic change of type ChangedAssoEndIsNavigable (the affected element of such a change is an Association): one of the association ends of the association is changed from navigable to non-navigable, or from non-navigable to navigable. Referring to Figure 25 , the only navigable end of the association between classes store and Register (i.e., the class Register end) is refined into a nonnavigable end according to the AssoEndIsNavigableRef refinement type. A similar refinement has also taken place on the class sale end of the association between classes Register and sale and on the class ProductDescription end of the association between class ProductDescription and SalesLineItem.

Once such an atomic change has been detected, a refinement, and corresponding traceability links can be specified: Figure 29 (b). The refinement's unique traceability link is between the original association (i.e., the affectedElement of the atomic change) and the refined association, e.g., between the association between classes store and Register in Figure 25 (a) and the association between classes store and Register in Figure 25 (b). Similarly to the previous refinement, the refined association in the refined model is identified by looking for the association end being refined (referred to as refAssoEnd) in the refined model. The class owning the association end (Property) has the same name as the class owning the original association end (referred to as origAssoEnd), the class owning the other association end of the association has the same name as the class owning the other association end of the original association, and the number of the navigableownedEnd of the association is changed, in order to establish a new traceability link corresponding to the AssoEndIsNavigableRef refinement. 


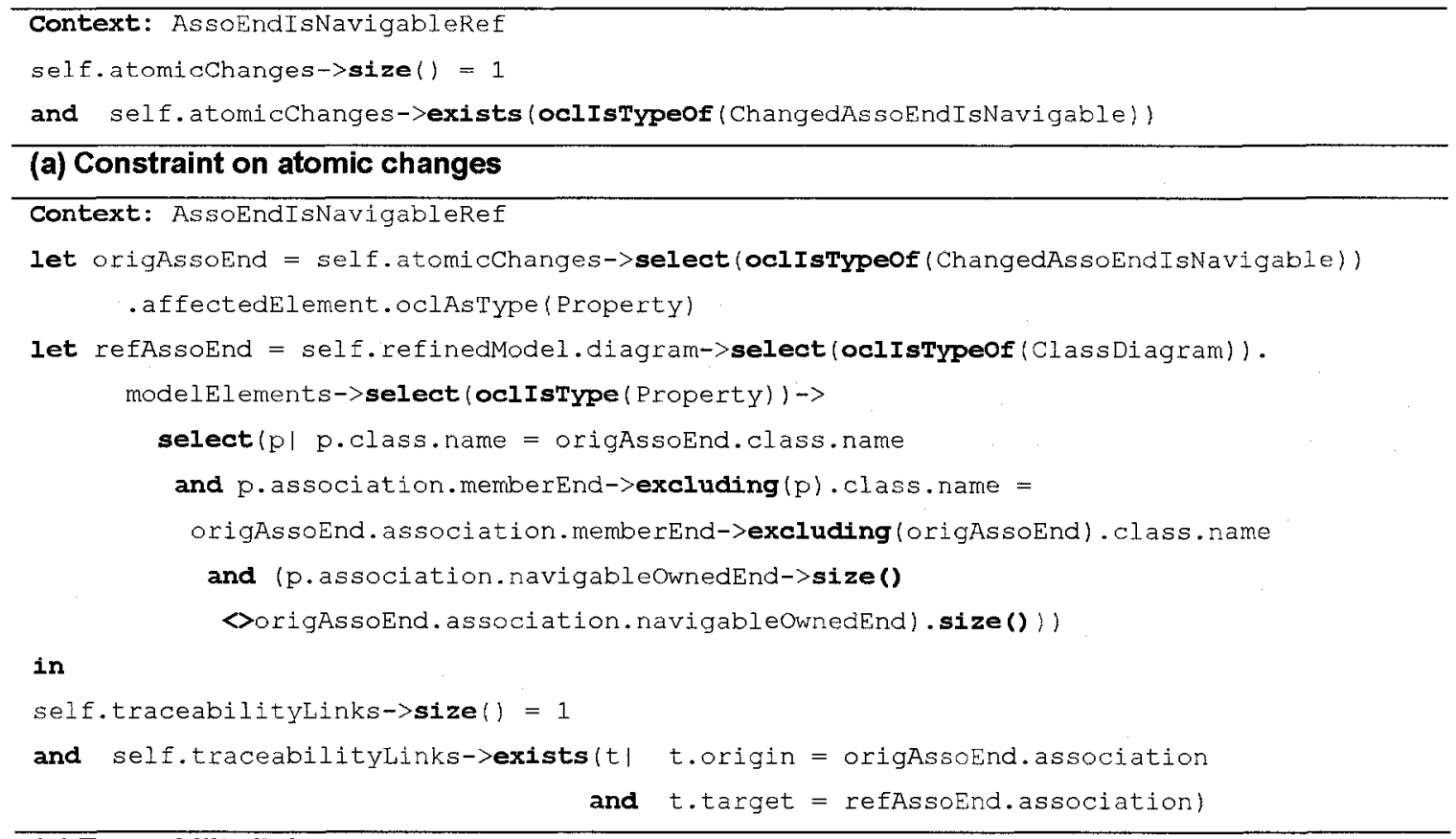

(b) Traceability links

Figure 29 Refinement AssoEndIsNavigableRef

\subsection{AssoEndTypeRef}

An AssoEndTyperef refinement is simple as it is detected, as specified in Figure 30 (a), from a single atomic change of type ChangedAs soEndType (the affected element of such a change is an Association). Referring to Figure 25, the association between classes ProductCatalog and ProductDescription is refined into a composition association according to the AssoEndTypeRef refinement type: the aggregation type of one of the association ends of the association is changed. A similar refinement has also taken place on the association between classes Sale and SalesLineItem.

Once such an atomic change has been detected, a refinement, and corresponding traceability links can be specified: Figure 30 (b). The refinement's unique traceability link is between the original association (i.e., the affectedElement of the atomic change) and the refined association, e.g., between the association between classes ProductCatalog and ProductDescription in Figure 25 (a) and the association between classes Productcatalog and ProductDescription in Figure 25 (b). Similarly to the previous refinement, the refined association in the refined model is 
identified by looking for the association end being refined (referred to as refAssoEnd) in the refined model. The class owning the association end (Property) has the same name as the class owning the original association end (referred to as origAssoEnd), the class owning the other association end of the association has the same name as the class owning the other association end of the original association, and the AggregationKind of the association is changed, in order to establish a new traceability link corresponding to the AssoEndType Ref refinement.

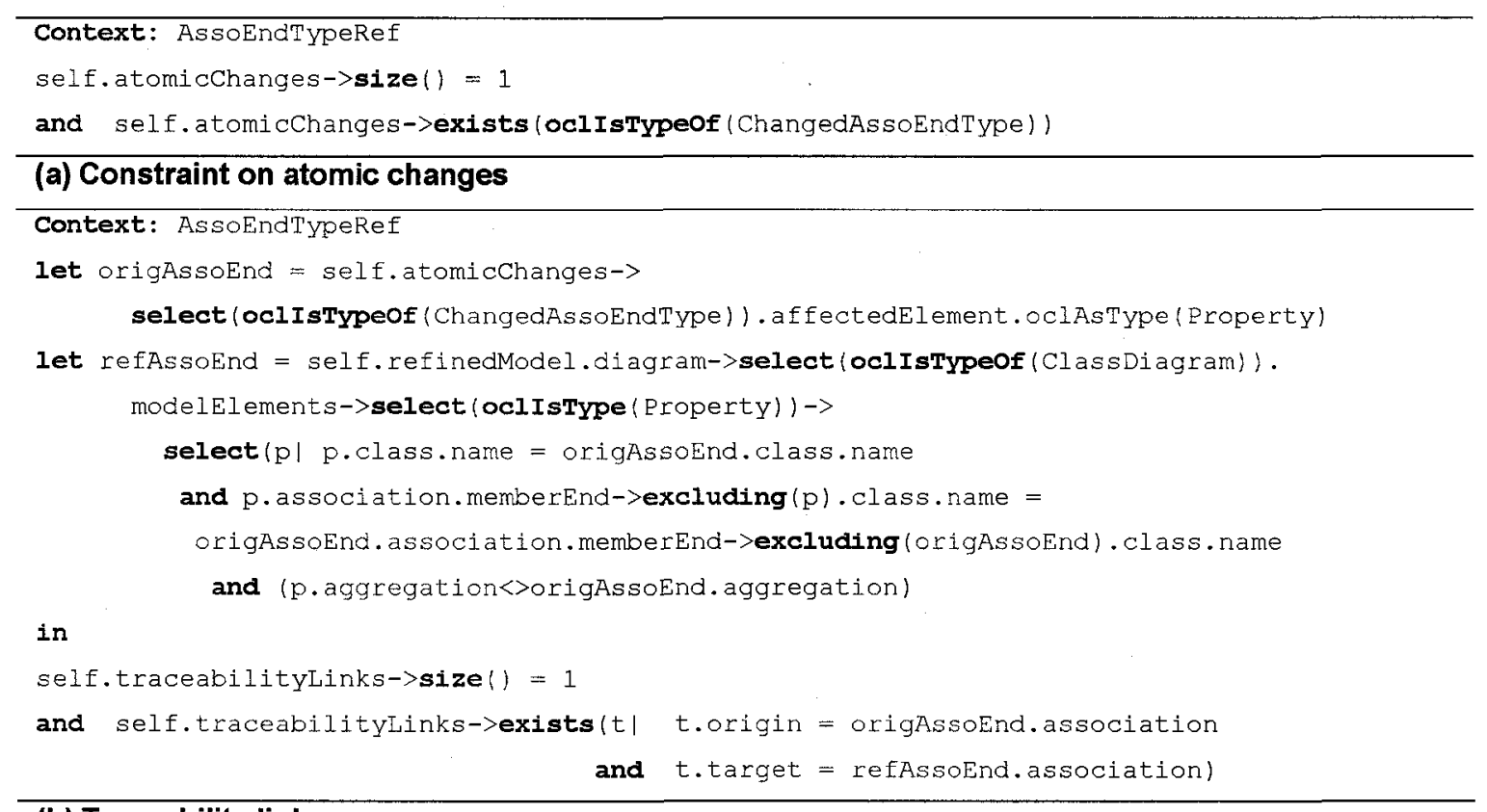

(b) Traceability links

Figure 30 Refinement AssoEndTypeRef 


\section{CONCLUSION}

UML models are, for typical systems, very complex artifacts. Modern development practices are iterative and rely on the stepwise refinement of analysis models into increasingly detailed design models, all the way down to implementation. However, changes are typically taking place at the same time as refinements and this results into a complex change management problem. How, under those conditions, can one ensure that models at different levels of abstractions remain consistent, especially in a context where changes and refinements are performed by different individuals in the context of large teams?

This thesis proposes a methodology and automation strategy. It is based on a careful classification of changes and refinements (capturing the changes' intent) in UML models and an automated identification of refinements based on detected changes. For each refinement, traceability links are then automatically established and can then be used to control the impact of changes in more abstract models on more refined models. We refer to this process as Vertical Impact Analysis. This work complements and extends earlier work on the impact analysis across UML diagrams within the same model, referred to as Horizontal Impact Analysis. Another contribution of this thesis is of methodological nature: we provide a way, which is based on metamodeling and constraints in the Object Constraint Language (OCL), to formally define change types, relate them to change intents (refinements) and formally specify the derivation of traceability links. This approach, while allowing for a rigorous and formal specification, has the advantage of facilitating the automation (the metamodel and constraints are a starting point for tool design) and is implementable using available, industry-strength modeling technology.

The thesis is only a first step as it only considers VIA for class diagrams. Much more work is under way to extend the work to the complete set of UML diagrams. But a first case study, based on a refined design model that was proposed independently from this research, shows that our classification and algorithms are complete and can accommodate all present changes. 
As introduced in the conceptual metamodel (see Section 4.1.1), the mechanism we proposed supports not only class diagram, but also other diagrams, e.g., sequence diagram. In this work, only class diagram refinements are considered (i.e., classified and formalized). One of our future works is to extend the work to the complete set of UML diagrams, e.g., sequence diagram.

As shown in the metamodel for refinement (see Section 4.1.2), a composite refinement is composed of a series of atomic refinements and/or other composite refinements. Although we provide a way to model them, we have not addressed their identification, which will be part of our future work. One possible area of investigation is perhaps to identify constraints that may exist among the atomic refinements and/or composite refinements contained by the composite refinement.

We provide a hierarchical taxonomy of class diagram atomic refinements based on a careful and systematic study of the related literature (conference and journal articles, and text books) or according to our own experience of UML-based software development. We made an effort to be as systematic as possible; however this taxonomy is also expected to be refined over time.

We propose to adapt the approach proposed in [4] (i.e., horizontal impact analysis) for vertical impact analysis (see Section 4.1.5). However in order to get more precise and/or complete horizontal and vertical impact analysis results, the HIA approach proposed in [4] has to be extended as we discussed in Section 3.3.1.

As we mentioned in Section 4.4.2, there exists an order in which refinements should be identified, finding such an order is however part of our future work as we currently rely on a muti-pass heuristic: OCL rules identifying refinements are all evaluated once, twice, and so on, until no new traceability link is established.

In our work, an initial framework for performing vertical impact analysis is introduced. However, how to perform VIA based on the traceability links established between two models has not been sufficiently addressed. For example, we may establish more than one traceability links between two identical model elements of these two models because 
it is possible that more than one refinements have been performed on the same model element. When VIA is performed, we have to choose which traceability link to follow to identify impacts. How to choose traceability link(s) to follow, and whether different kinds of links have different impacts, will be one of our future works. Besides, how to combine HIA and VIA together is not sufficiently addressed and will be a future work as well. 


\section{REFERENCES}

[1] Arnold R. S. and Bohner S. A., "Impact analysis-Towards a framework for comparison," Proc. Conference on Software Maintenance, pp. 292-301, 1993.

[2] Bohner S. A. and Arnold R. S., "An Introduction to Software Change Impact Analysis," in S. A. Bohner and R. S. Arnold, Eds., Software Change Impact Analysis, IEEE Computer Society, pp. 1-25, 1996.

[3] Bohner S. A. and Arnold R. S., Software Change Impact Analysis, IEEE Computer Society Press, 1996.

[4] Briand L. C., Labiche Y., O'Sullivan L. and Sowka M., "Automated Impact Analysis of UML Models," Journal of Systems and Software, vol. 79 (3), pp. 339-352, 2006.

[5] Bruegge B. and Dutoit A. H., Object-Oriented Software Engineering Using UML, Patterns, and Java, Prentice Hall, 2nd Edition, 2004.

[6] Eclipse Foundation, Eclipse Java Development Tools (JDT), www.eclipse.org/jdt (Last accessed May 2006)

[7] Eclipse Foundation, Eclipse Modeling Framework, www.eclipse.org/emf/ (Last accessed May 2006)

[8] Eclipse Foundation, Eclipse Technology Project, www.eclipse.org/technology/index.php (Last accessed May 2006)

[9] Eclipse Foundation, Eclipse Tools Project, www.eclipse.org/tools/ (Last accessed May 2006)

[10] Eclipse Foundation, Plug-in Development Envinronment (PDE), www.eclipse.org/pde (Last accessed May 2006)

[11] Eclipse Foundation, UML2: EMF-Based UML 2.0 Metamodel Implementation, www.eclipse.org/uml2/ (Last accessed May 2006)

[12] Engels G., Heckel R., Kuster J. M. and Groenewegen L., "Consistency-Preserving Model Evolution through Transformation," Proc. Unified Modeling Language. Model Engineering, Concepts, and Tools. LNCS, 2460, pp. 212-26, 2002.

[13] Engels G., Kuster J. M. and Groenewegen L., "Consistent Interaction of Software Components," Proc. Integrated Design and Process Technology, 2002.

[14] Fowler M., Refactoring Improving The Design of Existing Code, The AddisonWesley Object Technology Series, Addison-Wesley, 1999. 
[15] Hnatkowska B., Huzar Z., Kuzniarz L. and Tuzinkiewicz L., "Refinement Relationship between Collaborations," Proc. Workshop on Consistency Problems in UML-based Software Development, UML?3, 2003.

[16] IBM-Rational: Rational Software Architect, 2005. www-306$\mathrm{ibm} . \mathrm{com} / \mathrm{software} / \mathrm{awdtools/architect/swarchitect/.}$

[17] Judson S. R., France R. B. and Carver R. H., "Supporting Rigorous Evolution of UML Models," Proc. IEEE International Conference on Engineering of Complex Computer Systems, pp. 128-137, 2004.

[18] Kleppe A., Warmer J. and Bast W., MDA Explained - The Model Driven Architecture: Practice and Promise, Addison-Wesley, 2003.

[19] Kroll P. and Kruchten P., The Rational Unified Process Made Easy: A Practitioner's Guide to the RUP, Addison-Wesley, 2003.

[20] Larman C., Applying UML and Patterns, Prentice-Hall, $3^{\text {rd }}$ Edition, 2004.

[21] Letelier P., “A Framework for Requirements Traceability in UML-Based Projects," Proc. International Workshop on Traceability in Emerging Forms of Software Engineering, in Conjunction with ASE, 2002.

[22] Li L. and Offutt A. J., "Algorithmic analysis of the impact of changes to objectoriented software," Proc. IEEE International Conference on Software Maintenance, pp. 171-184, 1996.

[23] Li L. and Offutt A. J., "Algorithmic analysis of the impact of changes to objectoriented software," George Mason University ISSE Department Technical Report ISSETR-96-02, 1996.

[24] Marciniak J. J., Encyclopedia of Software Engineering, Wiley-Interscience, 2nd Edition, 2001.

[25] Mens T., Software Engineering Terminology from http://staff.umh.ac.be/Mens. Tom/ (Last accessed 1 December 2004

[26] Mens T. and D'Hondt T., "Automating Support for Software Evolution in UML," Automated Software Engineering (Springer), vol. 7 (1), pp. 39-59, 2000.

[27] OMG, "UML 2.0 Superstructure Specification," Object Management Group, Final Adopted Specification ptc/03-08-02, 2003.

[28] OMG, OMG Unified Modeling Language Specification, version 2.0, http://www.omg.org/technology/documents/formal/uml.htm, October, 2004.

[29] Pender T., UML Bible, Wiley, 2003. 
[30] Pons C. and Kutsche R.-D., "Traceability across Refinement Steps in UML Modeling," Proc. Workshop on Software Model Engineering, in conjunction with $U M L^{\prime} 04,2004$.

[31] Pons C., Perez G., Giandini R. and Kutsche R., "Understanding Refinement and Specialization in the UML," Proc. 2nd International Workshop on MAnaging SPEcialization/Generalization HIerarchies., 2004.

[32] Selic B., "Using UML for Modeling Complex Real-Time Systems," Proc. ACM SIGPLAN Workshop on Languages, Compilers and Tools for Embedded Systems, LNCS 1474, pp. 250-260, 1998.

[33] Shen W., Lu Y. and Low W. L., "Extending the UML Metamodel to Support Software Refinement," Proc. Workshop on Consistency Problems in UML-Based Software Development, in conjunction with UML, 2002.

[34] von Knethen A. and Grund M., "QuaTrace: A Tool Environment for (Semi)Automatic Impact Analysis Based on Traces," Proc. International Conference on Software Maintenance, pp. 246-255, 2003. 


\section{Appendix A Metamodel}

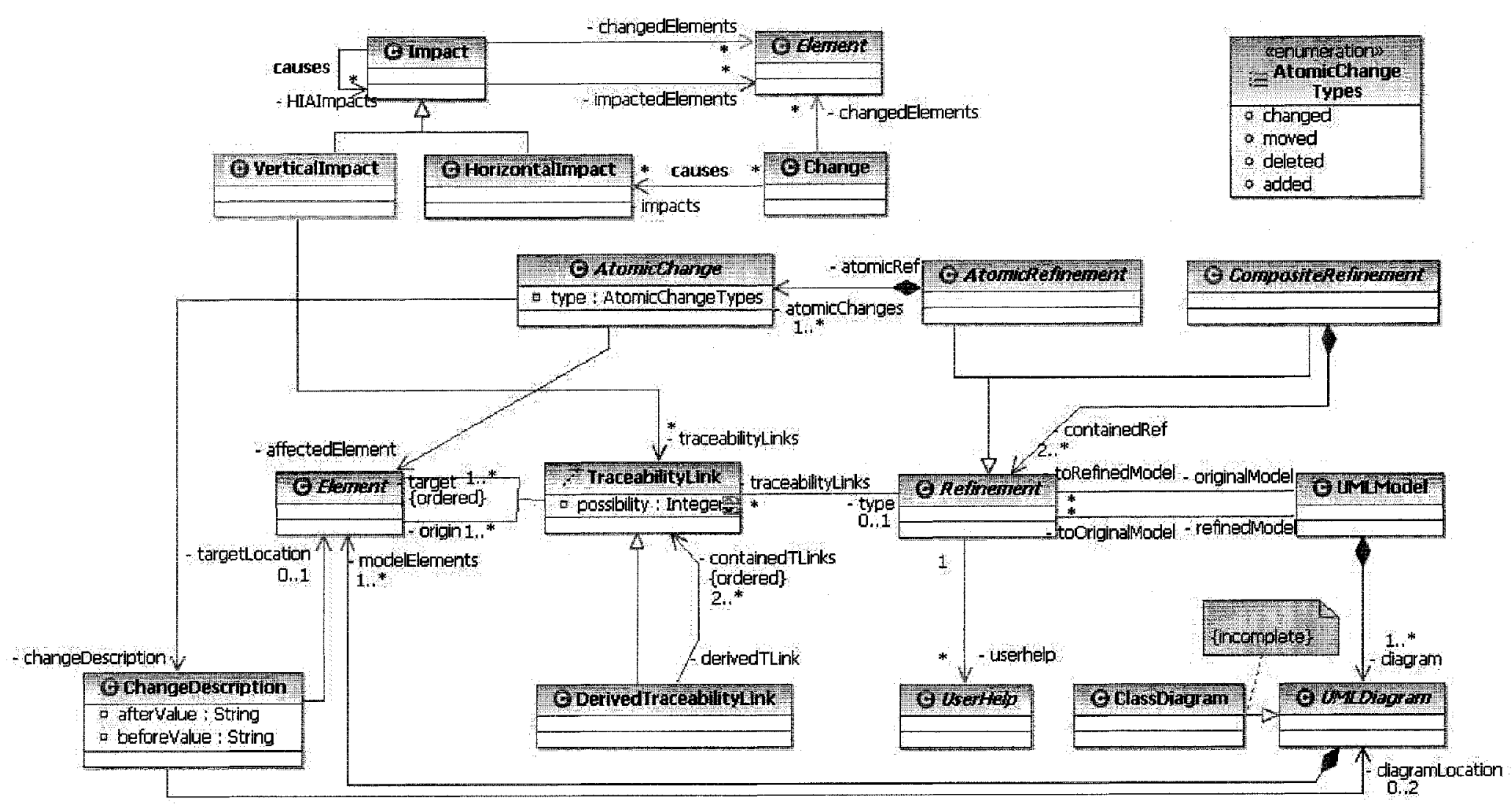

Figure 31 The complete metamodel 


\section{Appendix B Dictionary For Metamodel}

This appendix specifies the metaclasses of the metamodel presented in the Figure 8 , Figure 11, Figure 13, and Figure 14, listed in alphabetical order. The description for each metaclass is broken down into sub-sections corresponding to different aspects. The following sub-sections and conventions are used to specify a metaclass.

- The heading gives the name of the metaclass.

- The "Description" sub-section gives a brief textual description of the meaning of a metaclass.

- All the direct generalizations of the metaclass are listed in the "Generalizations" sub-section.

- A detailed description of the purpose of the metaclass is given in the "Purpose" sub-section.

- The "Attributes" sub-section lists each of the attributes that are defined for that metaclass. Each attribute is specified by its name, its type, and multiplicity. This is followed by a textual description of the purpose and meaning of the attribute.

- The "Associations" sub-section lists all the association ends owned by the metaclass. The format for these is the same as the one for attributes described above.

\section{B.1 AtomicChange}

\section{Description}

The metaclass AtomicChange represents the concept of atomic change defined in Section 2.3: a unitary and primitive change applied to a UML model that cannot be decomposed into other atomic changes.

\section{Generalizations}

- none

\section{Purpose}

Atomic changes are used to identify refinements. 


\section{Attributes}

- type: AtomicChangeTypes

It specifies the type of an atomic change. Refer to the description for AtomicChangeTypes for more information.

\section{Associations}

- changeDescription: ChangeDescription [1]

It specifies more detailed information of an atomic change except the type of the atomic change. Refer to the description for ChangeDescription for more information.

- affectedElement: Element [1]

It specifies the directly affected element of the atomic change.

\section{B.2 AtomicChangeTypes}

\section{Description}

AtomicChangeTypes specifies the different possible types of an atomic change. It is an enumeration type of the following literal values:

- changed Indicates that the affected element of an atomic change is changed.

- moved Indicates that the affected element of an atomic change is moved from one location to another location.

- deleted Indicates that the affected element of an atomic change is deleted.

- added Indicates that the affected element of an atomic change is added.

\section{Generalizations}

- none

\section{B.3 AtomicRefinement}

\section{Description}

The metaclass AtomicRefinement represents the concept of atomic refinement defined in Section 4.1.2: a refinement which can not be decomposed into other refinements. 


\section{Generalizations}

- Refinement

\section{Purpose}

Atomic refinements are used to represent those refinements which can not be decomposed into other refinements. Atomic refinements can be used to constitute composite refinements (refer to the description for CompositeRefinement).

\section{Attributes}

No attribute.

\section{Associations}

- atomicChanges: AtomicChange [1..*]

It indicates a group of atomic changes used to derive an atomic refinement.

\section{B.4 Change}

\section{Description}

A change can be any modification performed on the UML model.

\section{Generalizations}

- none

\section{Purpose}

A change is used during horizontal impact analysis to identify which elements are impacted and how these elements are impacted because of this change.

\section{Attributes}

No attribute.

\section{Associations}

- changedElement: Element [*]

It indicates the group of changed elements defined on a change. This concept is adapted from [4] in its Section 4.1.2. The criteria of identifying changed elements are given in [4] as well. 
- impacts: Impact $\left[{ }^{*}\right]$

It indicates the group of impacted elements resulting from the change. This concept is adapted from [4] in its Section 7.6.

\section{B.5 ChangeDescription}

\section{Description}

The metaclass ChangeDescription provides a detailed description of an atomic change.

\section{Generalizations}

- none

\section{Purpose}

See description.

\section{Attributes}

- aftervalue: String

It specifies the value after an atomic change of type changed (see AtomicChangeTypes) is made.

- beforevalue: String

It specifies the value before an atomic change of type changed (see AtomicChangeTypes) is made.

\section{Associations}

- targetLocation: Element [0..1]

This denotes the element, in the refined model, that owns the affected element (see affectedElements in class AtomicChange) for an atomic change of type moved (see AtomicChangeTypes) when this owner element is itself an element.

- diagramLocation: UMLDiagram [0..2]

This denotes the diagram, in the original or refined model, that owns the affected element (see affectedElements in class AtomicChange) for an atomic change of type moved (see AtomicChangeTypes) when this owner element is not an element (it is a diagram). 


\section{B.6 ClassDiagram}

\section{Description}

ClassDiagram is one of the subclasses of the metaclass UMLDiagram. It is used to represent a class diagram view of a UML model.

\section{Generalizations}

- UMLDiagram

\section{Purpose}

See description.

\section{Attributes}

No attribute.

\section{Associations}

No association.

\section{B.7 CompositeRefinement}

\section{Description}

The metaclass CompositeRefinement represents the concept of composite refinement defined in Section 4.1.2: refinements which are composed of several refinements which can be atomic refinements and/or composite refinements.

\section{Generalizations}

- Refinement

\section{Purpose}

The composite refinement is used to group refinements.

\section{Attributes}

No attribute.

\section{Associations}

- containedRef: Refinement [2..*] 
It specifies the refinements contained in a composite refinement.

\section{B.8 DerivedTraceabilityLink}

\section{Description}

DerivedTraceabilityLink is a traceability link derived from a sequence of traceability links.

\section{Generalizations}

- TraceabilityLink

\section{Purpose}

It is used to derive a traceability link from a sequence of traceability links: a link can be established between the origin of the first traceability link in the sequence and the target of the last traceability link in the sequence.

\section{Attributes}

No attributes.

\section{Associations}

- containedTLinks: TraceabilityLink [2..*]

It indicates a sequence (ordered association) of traceability links from which the derived traceability link is derived.

\section{B.9 Element}

\section{Description}

Element is an abstract metaclass. This class belongs to the UML 2.0 metamodel [28] and is therefore the bridge between our metamodel and the UML 2.0 metamodel.

\section{Generalizations}

- none

\section{Purpose}

In the UML 2.0 metamodel, an element is a constituent of a model. As such, it has the capability of owning other elements [28]. In our metamodel, an element is the bridge 
connecting our metamodel with the UML 2.0 metamodel. All the model elements of the UML model on which we perform our vertical impact analysis are instances of the metaclass Element.

\section{Attributes}

No attribute.

\section{Associations}

The associations in the UML 2.0 metamodel are not described here.

\section{B.10 HorizontalImpact}

\section{Description}

A Horizontal Impact is an impact caused by a change when horizontal impact analysis is performed.

\section{Generalizations}

Impact

\section{Purpose}

See description.

\section{Attributes}

No attribute.

\section{B.11 Impact}

\section{Description}

An Impact is caused by a particular change. This concept is adapted from [4].

\section{Generalizations}

- none

\section{Purpose}

See description. 


\section{Attributes}

No attribute.

\section{Associations}

- HIAimpacts: Impact [*]

It indicates the group of impacts caused by horizontal impact analysis. This association models the fact that an impact to a model element can result in other impacts.

- impactedElements: Element [*]

It indicates the group of impacted elements caused by an impact.

- changedElements: Element [*]

It indicates the group of changed elements caused by a change.

\section{B.12 Refinement}

\section{Description}

Refinement is an abstract metaclass. It is used as the common superclass for all metaclasses in our metamodel which represents the concept of refinement.

\section{Generalizations}

- none

\section{Purpose}

The metaclass Refinement is used to organize the different types of refinements as the abstract superclass of the composite design pattern.

\section{Attributes}

No attribute.

\section{Associations}

- traceabilityLinks: TraceabilityLink [*]

It indicates the traceability links that should be established for a refinement.

- originalModel: UMLModel [1]

It indicates the original model being refined. 
- refinedModel: UMLModel [1]

It indicates the refined model.

- userhelp: UserHelp [*]

It indicates the user help needed for identifying a refinement or establishing a traceability link.

\section{B.13 TraceabilityLink}

\section{Description}

TraceabilityLink is an association class which is used to represent the concept defined in Section 2.5: a relationship that describes the traceability connection between a model element in one UML model and a model element of another UML model.

\section{Generalizations}

- none

\section{Purpose}

Traceability links are used to establish relationships between two UML models at different levels of abstraction. Traceability links have to be established before performing vertical impact analysis.

\section{Attributes}

- possibility: Integer

It specifies the possibility of establishing the traceability link (see Section 4.1.4).

\section{Associations}

- target: Element [1..*]

- It indicates the model element of the refined model which traceability link points to. Notice that it is possible that an origin element is connected to more than one target elements through more than one traceability links.

- origin: Element [1..*] 
- It indicates the model element of the original model, which the traceability link originates from. Notice that it is possible that a target element is connected to more than one origin elements through more than one traceability links.

- type: Refinement [1]

It indicates the refinement for which the traceability link is established.

\section{B.14 UMLDiagram}

\section{Description}

UMLDiagram is an abstract metaclass, which is used as the superclass for all metaclasses in our metamodel which represent different views of UML model (e.g., Class Diagram).

\section{Generalizations}

- none

\section{Purpose}

In our metamodel, UMLDiagram is used to represent different views of UML model on which refinements are performed.

\section{Attributes}

No attribute.

\section{Associations}

- modelelements: Element [1..*]

It indicates the model elements owned by the UML diagram.

\section{B.15 UMLModel}

\section{Description}

UMLModel is a metaclass used to group UML diagrams. We assume that the diagrams contained in a UML model are consistent. From the aspect of the software lifecycle, this group of diagrams should be in the same phase of the lifecycle.

\section{Generalizations}

- none 


\section{Purpose}

See description.

\section{Attributes}

No attribute.

\section{Associations}

- toRefinedModel: Refinement [*]

It indicates the refinements which relate the model to its refined model(s). The rolename denotes that the model is connected to its refined model through the refinements.

- tooriginalModel: Refinement [*]

It indicates the refinements which relate the model to its original model(s). The rolename denotes that the model is connected to its original model through the refinements.

- diagram: UMLDiagram [1..*]

It indicates the diagrams contained in the UML model.

\section{B.16 UserHelp}

\section{Description}

UserHelp is an abstract metaclass. It represents the user help that may be required for identifying some refinements or establishing the traceability links for these refinements.

\section{Generalizations}

- none.

\section{Purpose}

See description.

\section{Attributes}

No attribute.

\section{Associations}

No attribute. 


\section{B.17 VerticalImpact}

\section{Description}

A Verticalimpact is an impact caused by a change through traceability links when vertical impact analysis is performed.

\section{Generalizations}

Impact

\section{Purpose}

See description.

\section{Attributes}

No attribute.

\section{Associations}

- traceabilityLinks: TraceabilityLink [*]

It indicates all traceability links between two UML models on which vertical impact analysis is performed. 


\section{Appendix C Example Atomic Changes}

This appendix provides a list of example atomic changes. The affectedElement and/or changeDescription are indicated for each example.

\section{C.1 Added}

1. AddedClass

Example: Added classA to classDiagramA

affectedElement: classA

diagramLocation: classDiagramA

2. AddedAttribute

Example: Added attributeA to classA

affectedElement: attributeA

3. Addedoperation

Example: Added operationA to classA

affectedElement: operationA

4. AddedAssociation

Example: Added association A between class A and classB

affectedelement: associationA

5. AddedAssociationclass

Example: Added associationClassA between classA and classB

affectedElement: associationClass A

6. AddedGeneralization

Example: Added generalization between class A (superclass) and classB (subclass) affectedElement: <generalization>

7. AddedInterface

Example: Added interfaceA to classDiagramA

affectedElement: interfaceA

diagramLocation: classDiagramA

8. AddedDependency

Example: Added dependency between classA (supplier) and classB (client) affectedElement: <Dependency>

\section{C.2 Deleted}

1. DeletedClass

Example: Deleted classA from classDiagramA

affectedElement: classA

diagramLocation: classDiagramA 
2. Deletedattribute

Example: Deleted attributeA from classA

affectedElement: attributeA

3. Deletedoperation

Example: Deleted operationA from classA

affectedElement: operationA

4. DeletedAssociation

Example: Deleted association A between classA and classB

affectedElement: associationA

5. DeletedAssociationclass

Example: Deleted associationClassA between classA and classB

affectedElement: associationClassA

6. DeletedGeneralization

Example: Deleted generalization between classA (superclass) and classB (subclass) affectedElement: <generalization>

7. Deletedinterface

Example: Deleted interfaceA from classDiagramA

affectedElement: interfaceA

diagramLocation: classDiagramA

8. DeletedDependency

Example: Deleted dependency between classA (supplier) and classB (client)

affectedElement: <Dependency>

\section{C.3 Changed}

\section{C.3.1 ChangedClass}

1. Renameclass

Example: Rename classA to classB

affectedElement: classA

beforeValue: classA

afterValue: classB

2. ChangedClassIsAbstract

Example: Changed classA's property isAbstract from false to true

affectedElement: classA

beforevalue: false

afterValue: true 
3. ChangedClassVisibility

Example: Changed classA's property visibility from public to private affectedElement: classA

beforeValue: public

aftervalue: private

4. ChangedClassisActive

Example: Changed classA's property isActive from false to true affectedElement: classA

beforevalue: false

aftervalue: true

5. ChangedClassisleaf

Example: Changed classA's property isLeaf from false to true

affectedElement: classA

beforeValue: false

aftervalue: true

\section{C.3.2 ChangedAttribute}

1. RenameAttribute

Example: Rename attribute1 to attribute2

affectedElement: attribute 1

beforeValue: attribute1

afterValue: attribute2

2. ChangedAttritype

Example: Changed attributeA's type from String to Integer

affectedElement: attributeA

beforevalue: String

aftervalue: Integer

3. ChangedAttriMultiplicity

Example: Changed attributeA's multiplicity from 1 to *

affectedElement: attributeA

beforevalue: 1

afterValue: *

4. ChangedAttrivisibility

Example: Changed attributeA's visibility from public to private affectedElement: attributeA

beforevalue: public

afterValue: private 
5. ChangedAttriInitialValue

Example: Changed attributeA's initialValue from $a$ to $b$

affectedElement: attributeA

beforevalue: a

aftervalue: $b$

6. ChangedAttriIsordered

Example: Changed attributeA's property isOrdered from false to true affectedElement: attributeA

beforevalue: false

aftervalue: true

\section{C.3.3 ChangedOperation}

1. Renameoperation

Example: Rename operation 1 to operation2

affectedElement: operation 1

beforevalue: operation 1

afterValue: operation2

2. ChangedoprIsAbstract

Example: Changed operationA's property isAbstract from false to true affectedElement: operationA

beforevalue: false

aftervalue: true

3. ChangedoprParameter

Example: Changed one of parameters of the operationA

affectedElement: operationA

4. ChangedoprVisibility

Example: Changed operationA's visibility from public to private

affectedElement: operationA

beforevalue: public

afterValue: private

5. ChangedoprReturnType

Example: Changed operationA's return type from $a$ to $b$

affectedElement: operationA

beforevalue: a

afterValue: b

\section{C.3.4 ChangedAssociation}


1. ChangedAssoEndType

Example: Changed associationA's association end classA's property aggregation from none to composite affectedElement: associationA's association end beforeValue: none aftervalue: composite

2. ChangedAssoEndMultiplicity

Example: Changed associationA's association end classA's property multiplicity from 1 to *

affectedElement: associationA's association end

beforeValue: 1

afterValue: *

3. ChangedAssoEndIsNavigable

Example: Changed associationA's association end classA's property isNavigable from false to true

affectedElement: associationA's association end

beforevalue:false

aftervalue: true

\section{C.3.5 ChangedGeneralization}

1. ChangedGenIsSubstitutable

Example: Changed generalization's property isSubstitutable from false to true affectedElement: <Generalization>

beforevalue:false

aftervalue: true

\section{C.3.6 ChangedInterface}

1. RenameInterface

Example: Rename interfaceA to interfaceB

affectedElement: interfaceA

beforevalue: interfaceA

aftervalue: interfaceB

2. ChangedInterfaceIsAbstract

Example: Changed interfaceA's property isAbstract from false to true affectedElement: interface $A$

beforeValue: false

aftervalue: true

3. ChangedInterfaceVisibility

Example: Changed interfaceA's property visibility from public to private affectedElement: interfaceA

beforevalue: public

afterValue: private 
4. ChangedInterfaceIsActive

Example: Changed interfaceA's property isActive from false to true

affectedElement: interfaceA

beforevalue: false

aftervalue: true

5. ChangedInterfaceIsLeaf

Example: Changed interfaceA's property isLeaf from false to true

affectedElement: interfaceA

beforeValue: false

aftervalue: true

\section{C.3.7 ChangedDependency}

1. RenameDependency

Example: Rename dependencyA to dependencyB

affectedElement: dependencyA

beforevalue: dependencyA

afterValue: dependencyB

\section{C.4 Moved}

1. MovedAttribute

Example: Moved attributeA from classA to classB

affectedElement: attributeA

targetLocation: classB

2. Movedoperation

Example: Moved operationA from classA to classB

affectedElement: operationA

targetLocation: classB

3. RelocateAssociation

Example: associationA has two association ends: class A and classB. Moved one association end from class $B$ to class $C$

affectedElement: associationA

targetLocation: classC

4. RelocateGeneralization

Example: the generalization connects classA and classB. Moved one end from classB to classC

affectedElement: <Generalization $>$

targetLocation: classC

5. RelocateDependency

Example: the dependencyA connects classA and classB. Moved one end from classB to classC

affectedElement: dependencyA 
targetLocation: classC 


\section{Appendix D Taxonomy For Atomic Changes}

Figure 32 shows the top level of taxonomy which contains eight concrete atomic changes of type added, eight concrete atomic changes of type deleted, five concrete atomic changes of type moved, and seven abstract atomic changes of type changed. For these abstract changes of type changed, sub-classifications are provided in Figure 33, Figure 34, Figure 35, Figure 36, Figure 37, Figure 38, and Figure 39. A textual description of each concrete atomic change is provided as follows.

1. AddedClass: the addition of a class.

2. AddedAttribute: the addition of an attribute to a class.

3. Addedoperation: the addition of an operation to a classifier.

4. AddedAssociation: the addition of an association between two classes.

5. AddedGeneralization: the addition of a generalization relationship between two classes.

6. AddedInterface: the addition of an interface.

7. AddedDependency: the addition of a dependency relationship.

8. AddedAssociationclass: the addition of an association class.

9. DeletedClass: the deletion of a class.

10. DeletedAttribute: the deletion of an attribute from a class.

11. Deletedoperation: the deletion of an operation from a classifier.

12. DeletedAssociation: the deletion of an association between two classes.

13. DeletedGeneralization: the deletion of a generalization relationship between two classes.

14. Deletedinterface: the deletion of an interface.

15. DeletedDependency: the deletion of a dependency relationship.

16. DeletedAssociationClass: the deletion of an association class.

17. MovedAttribute: the move of an attribute between two classifiers.

18. Movedoperation: the move of an operation between two classifiers.

19. Relocateassociation: the move of one of the association ends of an association from a classifier to another classifier. 
20. RelocateGeneralization: the move of the general classifier or specializing classifier of a generalization relationship from a classifier to another classifier.

21. RelocateDependency: the move of the supplier end or client end of a dependency relationship between two model elements.

22. RenamedClass: the change of a class name.

23. ChangedClassvisibility: the change of property visibility [27] of a class.

24. ChangedClassisAbstract: the change of property isAbstract [27] of a class.

25. ChangedClassIsActive: the change of property isActive [27] of a class.

26. ChangedClass IsLeaf: the change of property isLeaf [27] of a class.

27. RenamedAttribute: the change of an attribute's name.

28. Changedattritype: the change of an attribute's type.

29. ChangedAttrimultiplicity: the change of property multiplicity [27] of an attribute.

30. ChangedAttrivisibility: the change of property visibility of an attribute.

31. ChangedAttriInitialValue: the change of property default [27] of an attribute.

32. ChangedattriIsOrdered: the change of property isordered [27] of an attribute.

33. Renamedoperation: the change of an operation's name.

34. ChangedOprIsAbstract: the change of property is Abstract of an operation.

35. ChangedoprParameter: the change of an operation's parameter list.

36. ChangedoprVisibility: the change of property visibility of an operation.

37. ChangedoprReturnType: the change of an operation's return type.

38. ChangedAssoEndType: the change of property aggregation [27] of an association end.

39. ChangedAssoEndMultiplicity: the change of property multiplicity of an association end.

40. ChangedAs SoEndIsNavigable: the change of the navigability of an association end

41. ChangedGenIsSubstitutable: the change of property issubstitutable [27] of a generalization relationship.

42. RenamedInterface: the change of an interface name. 
43. ChangedInterfaceIsAbstract: the change of property isAbstract of an interface.

44. ChangedClassvisibility: the change of property visibility of an interface.

45. ChangedClassisActive: the change of property isActive of an interface.

46. ChangedClassisleaf: the change of property isLeaf of an interface.

47. RenamedDependency: the change of a dependency relationship name. 


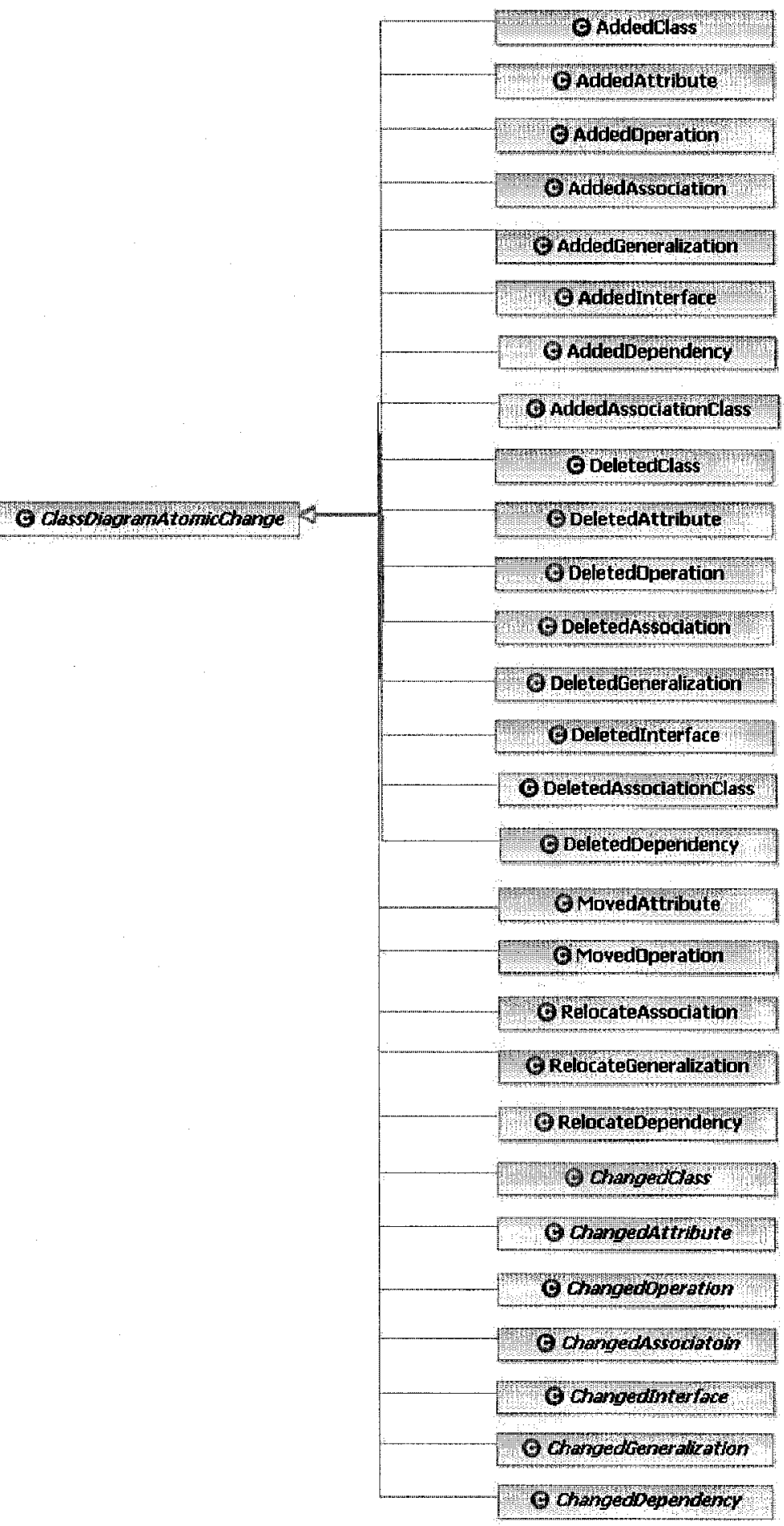

Figure 32 Complete Taxonomy for Atomic Changes - Top Level 


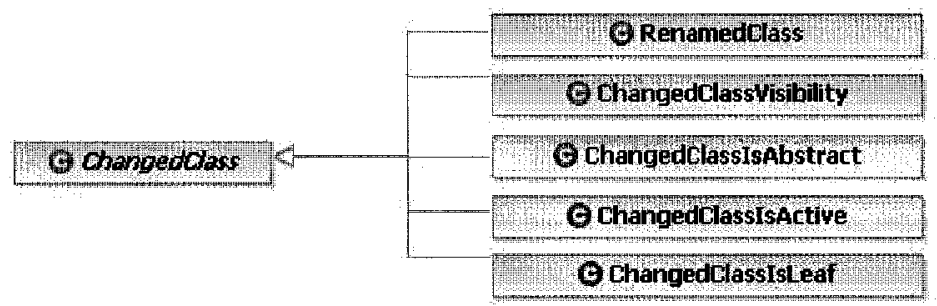

Figure 33 Taxonomy for Atomic Changes of Type ChangedClass

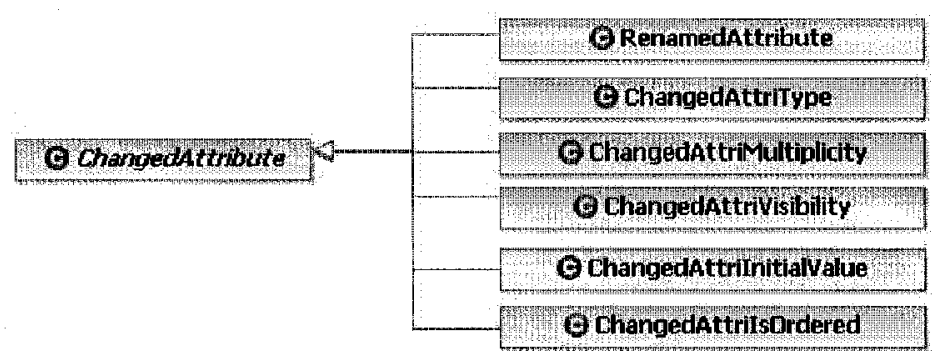

Figure 34 Taxonomy for Atomic Changes of Type ChangedAttribute

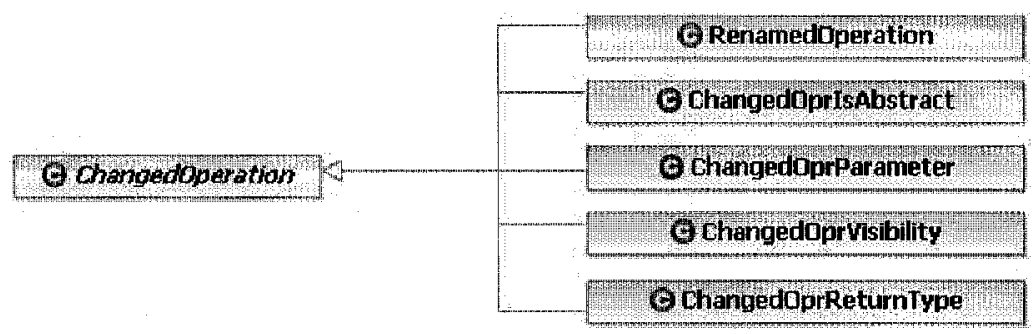

Figure 35 Taxonomy for Atomic Changes of Type Changedoperation

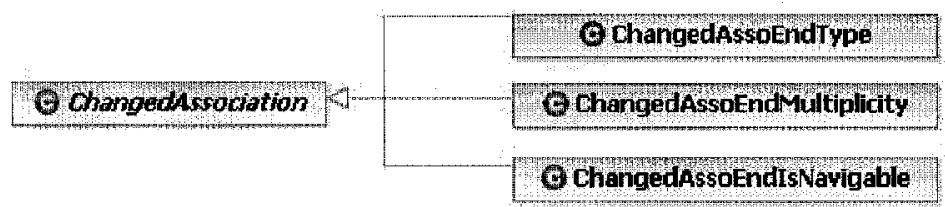

Figure 36 Taxonomy for Atomic Changes of Type ChangedAssociation

\begin{tabular}{|c|c|}
\hline CWhortheneralization & O changedisenIssubstitutable \\
\hline
\end{tabular}

Figure 37 Taxonomy for Atomic Changes of Type ChangedGeneralization 


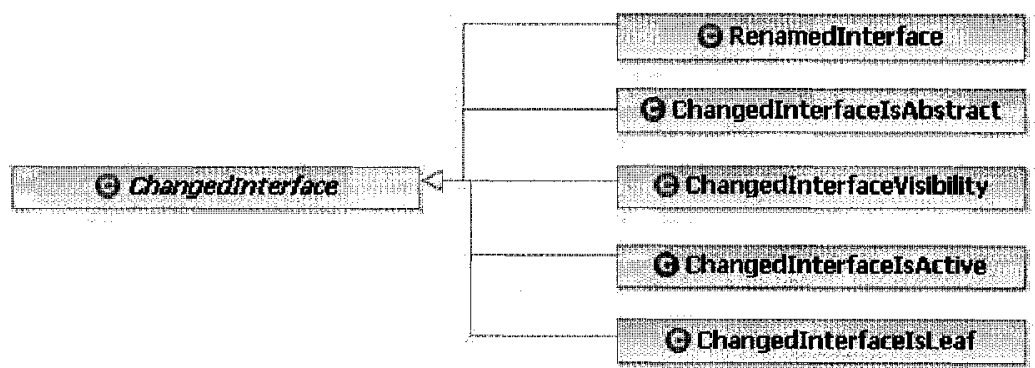

Figure 38 Taxonomy for Atomic Changes of Type ChangedInterface

O changedbebendency o ORenamedDependency

Figure 39 Taxonomy for Atomic Changes of Type ChangedDependency 


\section{Appendix E Taxonomy and formalization for refinements}

Since a class diagram is a diagram showing a collection of classes and interfaces, along with the collaborations and relationships among classes and interfaces, we can classify class diagram refinements according to the way these classes, interfaces and relationships are changed and transformed. Classifying class diagram refinements this way helps ensure the completeness of the classification. Figure 40 shows the top level of the taxonomy which contains eight abstract refinements. For each abstract refinement, a second level of classification is provided in the following subsections.

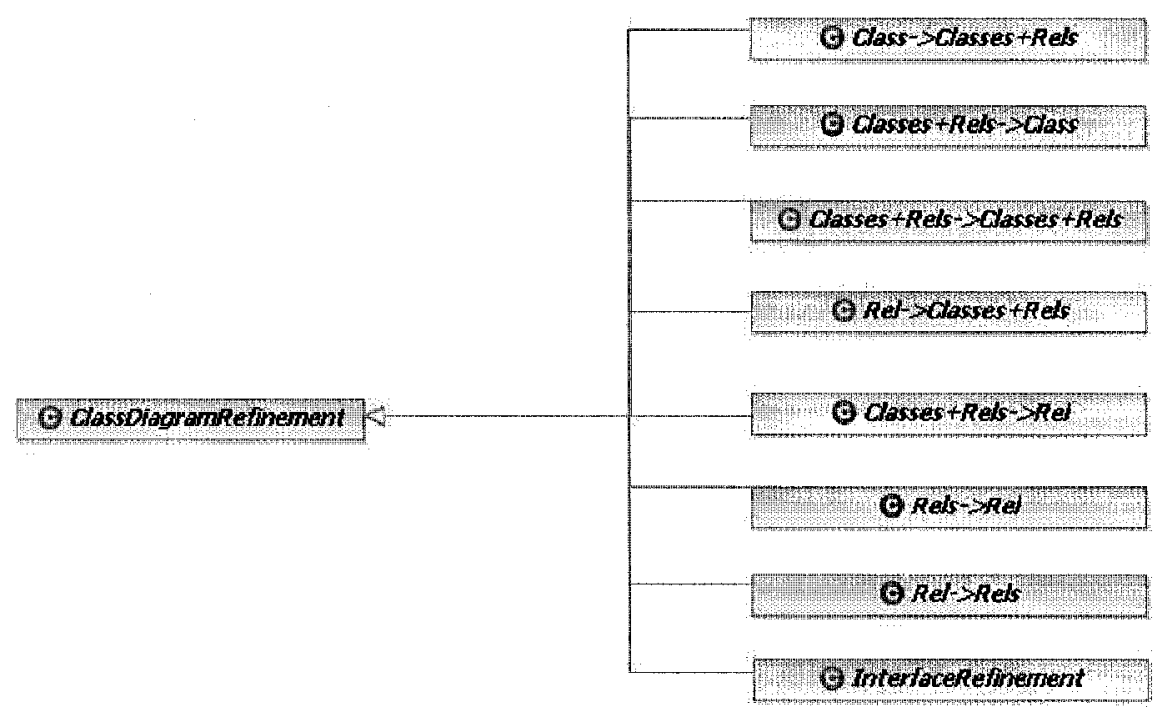

Figure 40 Taxonomy for Refinements - Top Level

Note that we do not have any Classes->Class not Class->Classes abstract classes in our refinement taxonomy. The former family of refinement would refine several classes into a class, whereas the latter would refine a single class into several classes. It is impossible to have these kinds of refinements since the definitions would imply that the classes are isolated without relationship to connect them with other model elements of the diagram. For instance, in the former refinement, the classes being refined into one class should be related by some relationships. The refinement would then be Classes + Rels- $>$ Class, which already appears in the taxonomy. 
Refinements Class->ClassestRels, ClassestRels->Class, Classes+Rels$>$ ClassestRels, Rel->ClassestRels, Classes+Rels->Rel, Rels->Rel, Rel$>\operatorname{Rels}$, and InterfaceRefinement are further described in Sections E.1, E.2, E.3, E.4, E.5, E.6, E.7, and E.8, respectively.

\section{E.1 Class->Classes+Rels}

This refinement refines a single class into several classes and relationships. The general purpose of this kind of refinement is to extend or split the functionality of the class being refined by introducing more classes and relationships. Figure 41 provides a refined classification (i.e., second level of classification) involving seven types of concrete atomic refinements.

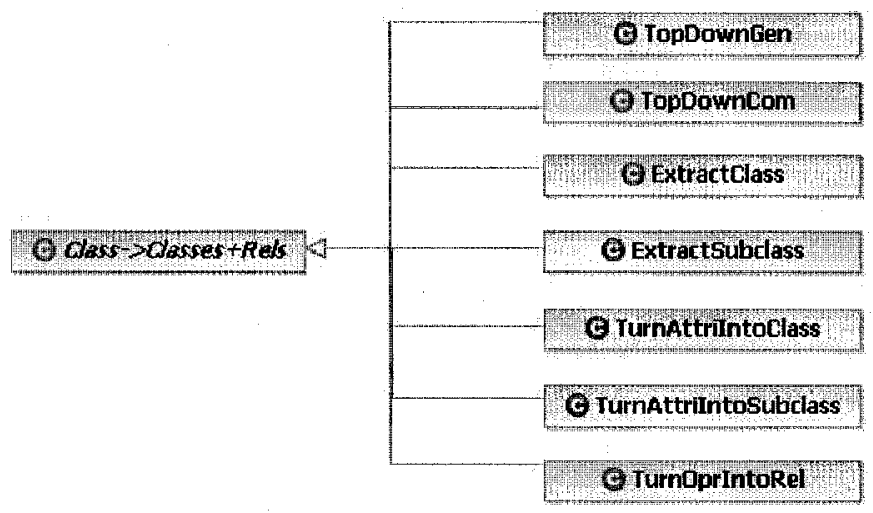

Figure 41 Taxonomy for Refinement of Type Class->Classes+Rels

A class can be refined into several classes and their relationships for three main reasons. First, the rationale is to detail the responsibility of one class (in the original model) by adding more classes and relationships. TopDownGen (Section E.1.1) and TopDownCom (Section E.1.2) belong to this group: TopDownGen introduces more classes using generalization, and TopDownCom introduces more classes using composition. A second possible reason is to split the responsibility of one class and distribute this responsibility over several (new) classes. ExtractClass (Section E.1.3) and TurnAttriIntoclass (Section E.1.5) as well as Extractsubclass (Section E.1.4) and TurnAttriIntosubclass (Section E.1.6) belong to this group: Extractclass and TurnAttriIntoclass extract some attributes and/or operations from the original class 
to form a new class that is associated with the original class; Extractsubclass and TurnAttriIntoSubclass extract some attributes and/or operations from the original class to form a subclass that specializes the original class. A third possible rationale is to refine an operation of the original class. TurnoprIntoRel (Section E.1.7) belongs to this group: TurnoprIntoRel refines an operation of the original class into a relationship which connects to the original class. 


\section{E.1.1 TopDownGen}

Description: Introduce subclasses to the class being refined using generalization to suggest general-specific relationships.

Specification: The class Originalclass of the original model is refined into the superclass Superclass and its subclass Subclass, as shown in Figure 42.

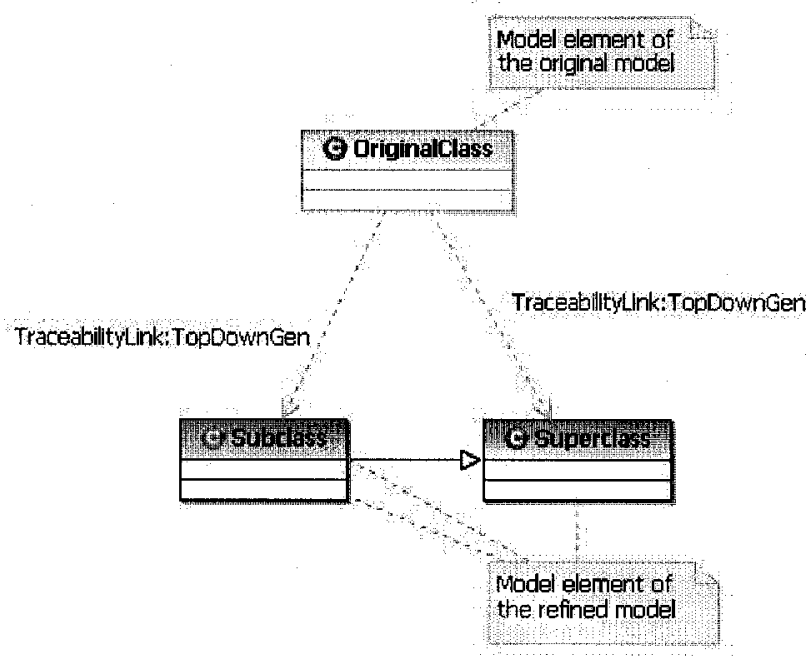

Figure 42 TopDownGen - Specification ${ }^{78}$

User's Intention: Detail the responsibility of the original class by introducing subclasses using generalization, because these subclasses, though similar, have significant differences in the interface or services they provide.

Atomic Changes: Two kinds of atomic changes are required to derive the refinement: AddedClass and AddedGeneralization. Note that we only account for the situation in which only one subclass is added. If more than one subclass is added, more than one occurrence of the refinement of type TopDownGen is identified. The specification of

\footnotetext{
7 The two dependeny relationships with label TraceabilityLink: TopDownGen are presented in the specification class diagram, but it does not mean that these traceability links must be explicitly represented or manually established. It is just for the purpose of clear explanation. It is same for the rest of atomic refinements in this appendix.

${ }^{8}$ The model elements in the refined model are highlighted in order to distinguish them from the model elements of the original model.
} 
refinement TopDownGen, i.e., the characterization of its atomic changes and their relations, is shown as the OCL invariant for class TopDownGen in Figure 43 (a).

Traceability Links: Two traceability links have to be established: one between the original class and the superclass of the added generalization; one between the original class and the added subclass. These traceability links are specified in the OCL expression in Figure 43 (b).

User Help: none.

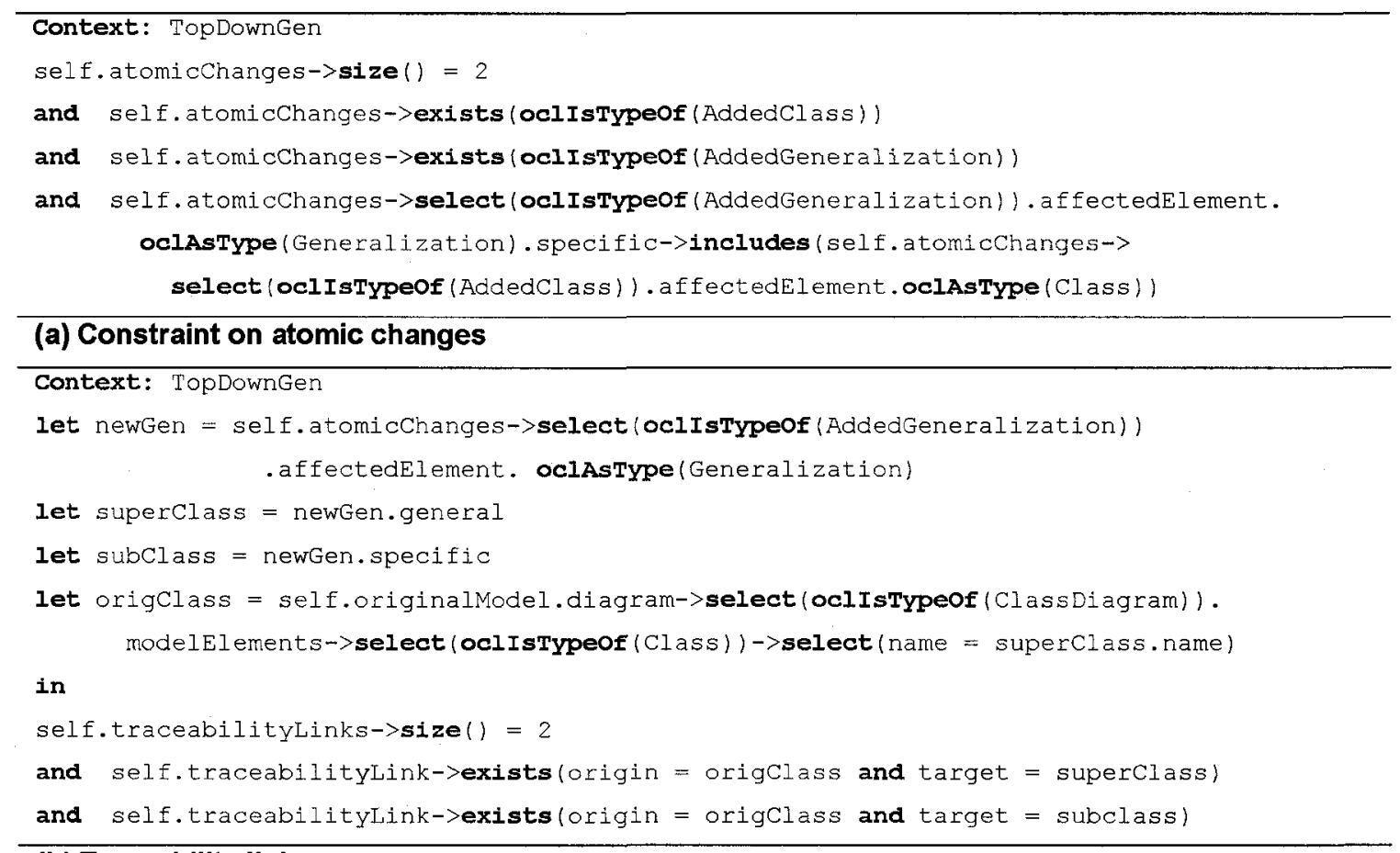

(b) Traceability links

Figure 43 Refinement TopDownGen

Example: The example shown in Figure 44 comes from [20]. The class Payment of the analysis model is refined into the abstract class Payment plus the three subclasses: CashPayment, CreditPayment and CheckPayment. Three traceability links are established. Notice that this example presents a composite refinement which contains two types of atomic refinements: ClassisAbstractRef which is derived from a single atomic change of type ChangedClassIsAbstract (performed once) and TopDownGen (performed three times). 


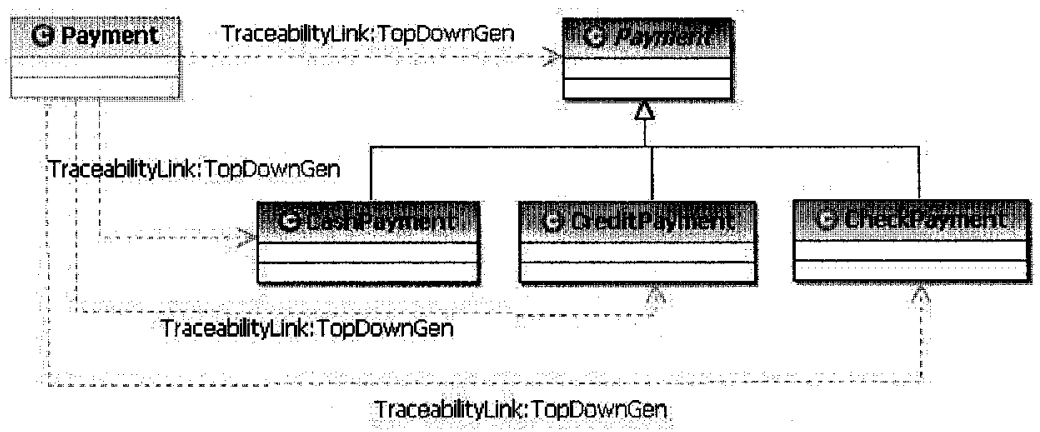

Figure 44 TopDownGen - Example 


\section{E.1.2 TopDownCom}

Description: Introduces new classes using composition associations to suggest wholepart relationships between the class being refined and the new added classes.

Specification: The class Originalclass of the original model is refined into the classes ComposedClass and Composingclass, as shown in Figure 45. The class Composedclass is connected to the new added class Composingclass by the composition association newCom.

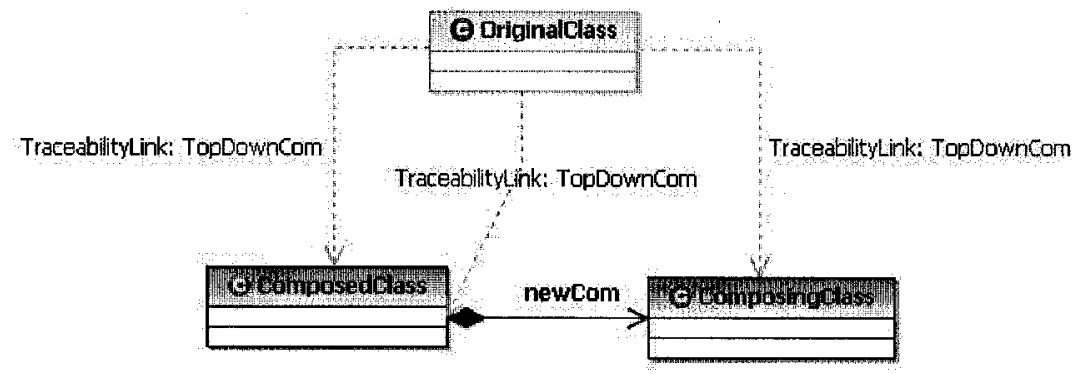

Figure 45 TopDownCom - Specification

User's Intention: Detail the responsibility of the original class by introducing new classes using composition associations to suggest whole-part relationships. The instance of the part (i.e., new class) belongs to only one composite instance (i.e., original class) at a time. The part must always belong to a composite and the composite is responsible for the creation and deletion of its parts.

Atomic Changes: Two kinds of atomic changes are required to derive the refinement: AddedClass and AddedAssociation, such that the association has a composition end and the non-composition part of the added association points to the added class. The specification of refinement TopDownCom is shown as the OCL invariant for class TopDownCom in Figure 46 (a).

Traceability Links: Three traceability links have to be established: one between the original class and the composing class of the added composition association; one between the original class and the composed class; one between the original class and the added 
composition association. These traceability links are specified in the OCL expressions in Figure 46 (b).

User Help: none.

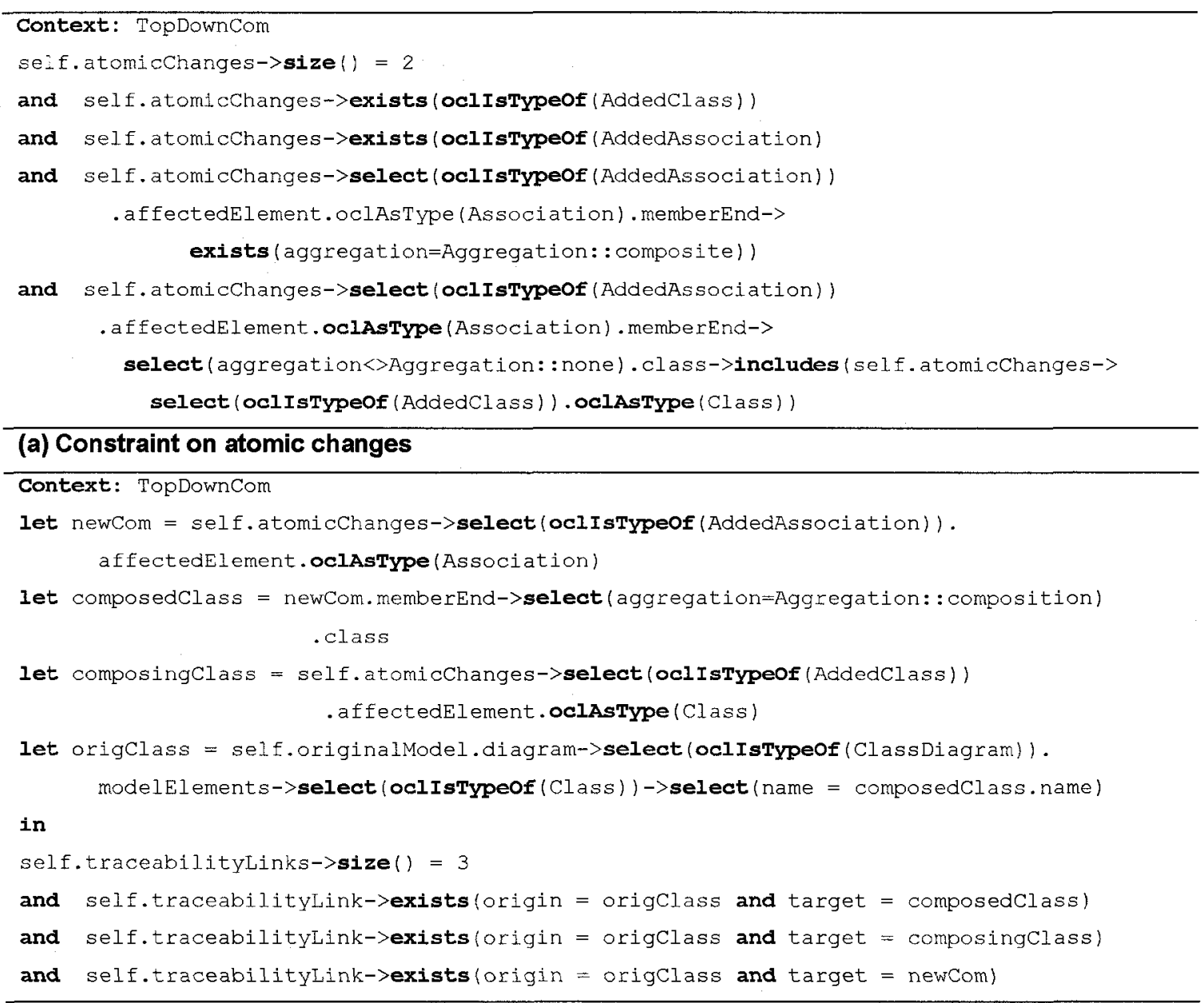

(b) Traceability links

Figure 46 Refinement TopDownCom

Example: Figure 47 presents an example. The new class Finger is added and connected to the class Hand by a composition association. Thus, an instance of the class Hand is composed of several instances of the class Finger. 


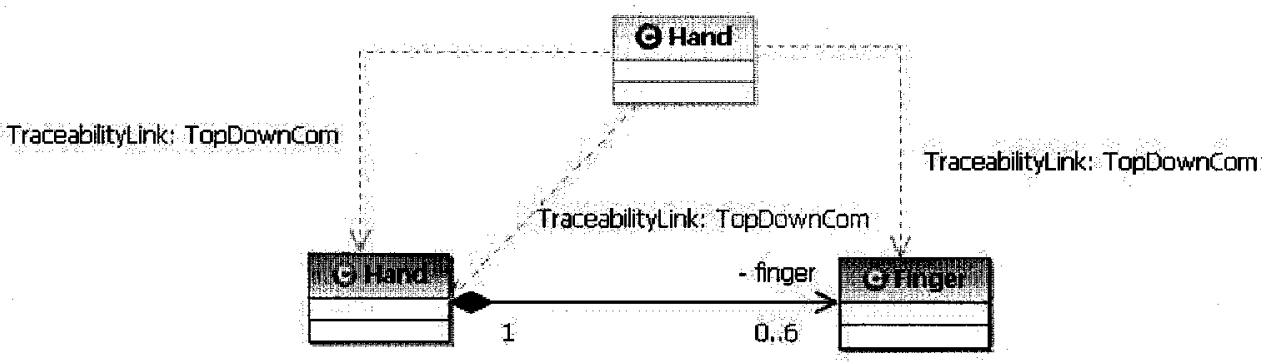

Figure 47 TopDownCom - Example 


\section{E.1.3 ExtractClass}

Description: Introduces a new class associated to the class being refined and moves some attributes and/or operations from the class being refined to the new class, thus splitting the responsibilities of the class being refined.

Specification: The attribute movedAttribute of the class Originalclass is moved into the newly added class NewClass and the new association newAsso is added to connect the classes RefinedClass and NewClass, as shown in Figure 48. Notice that the class Originalclass's operations may be moved to the class NewClass, even though we do not show this situation in Figure 48.

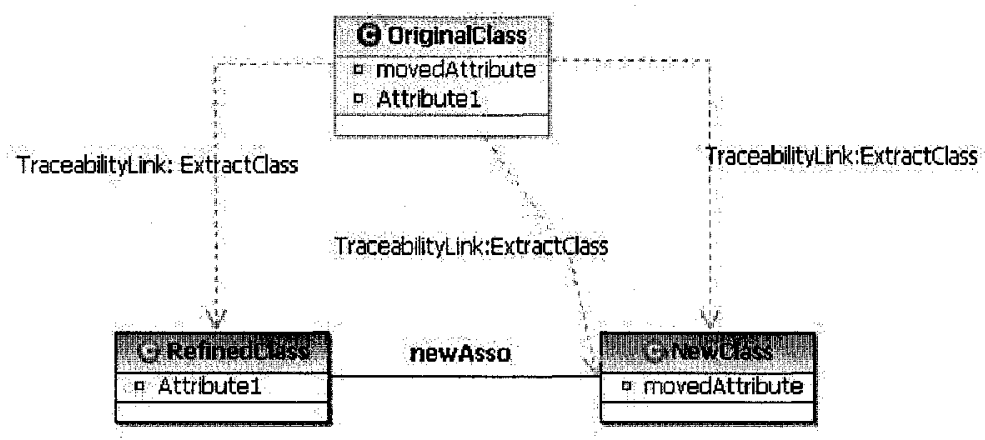

Figure 48 Extractclass - Specification

User's Intention: To split the responsibilities of the original class into two classes. When a class burdens many responsibilities and becomes too complicated, we may think of splitting this class into two classes so that each class handles a few clearer responsibilitities.

Atomic Changes: Three or four kinds of atomic changes may be used to derive the refinement: Addedclass, AddedAssociation (between the added class and the class which the attribute/operation has been moved from), and Movedattribute and/or Movedoperation (to the added class). However, it is not required to have both MovedAttribute and Movedoperation at the same time since sometimes only some attributes are moved or only some operations are moved. Figure 49 (a) only presents the case that only one MovedAttribute is included in the group of atomic changes in order 
to make the OCL expressions more concise. (Accounting for moved operations is very similar.)

Traceability Links: Three traceability links have to be established: one between the original class and the refined class; one between the original class and the newly added class; one between the original class and the newly added association. These traceability links are specified in the OCL expressions in Figure 49 (b). Note that we do not establish traceability links at the (moved) attribute or operation level since we consider that a change to the attribute (or operation) in the original model may result in changes to both the refined class and the added class in the target model. Future work on vertical impact analysis will look into whether this is.

User Help: none. 


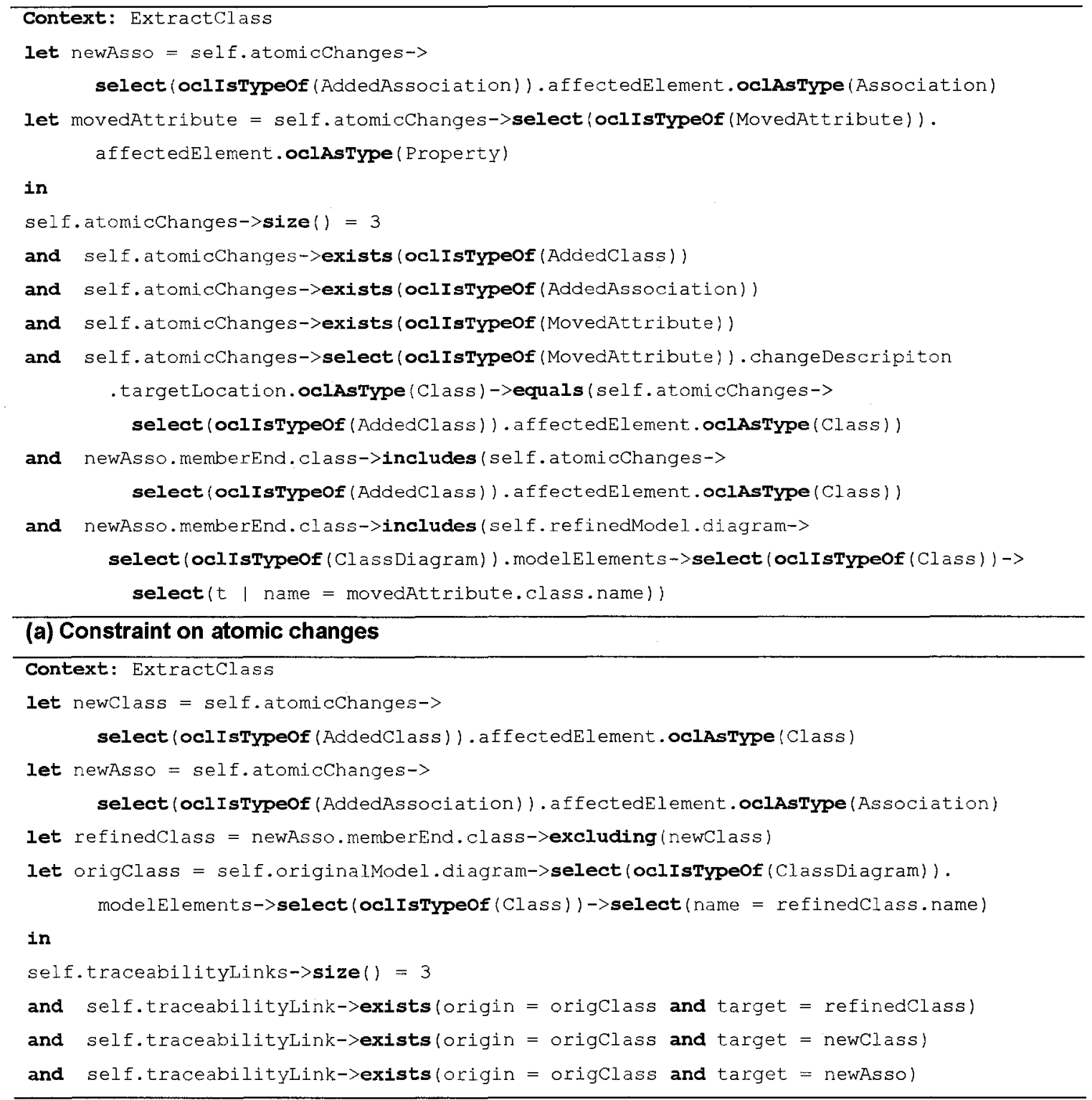

(b) Traceability links

Figure 49 Refinement Extractclass

Example: Figure 50 presents an example excerpted from [14]. The class Person in the original model is refined into the classes Person and TelephoneNumber and the association officeTelephone. The attributes officeAreaCode and officeNumber are moved from the class Person into the newly added class TelephoneNumber. Note that in this example, these two attributes are renamed after the refinement Extractclass is performed in which case a composite refinement is formed. 


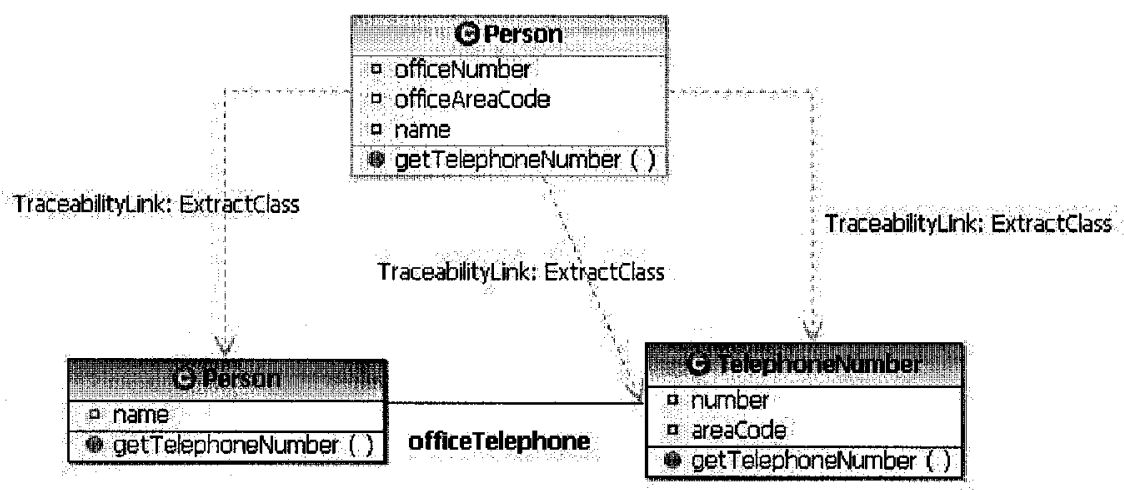

Figure 50 Extractclass - Example 


\section{E.1.4 ExtractSubclass}

Description: Introduces subclasses to the class being refined using generalization and moves some attributes and/or operations from the class being refined into the new subclasses.

Specification: As shown in Figure 51, the attribute movedAttribute of the class originalclass is moved into the newly added subclass NewSubclass. Note that the Originalclass's operations may be moved into the NewSubclass, even though we do not show this case in Figure 51.

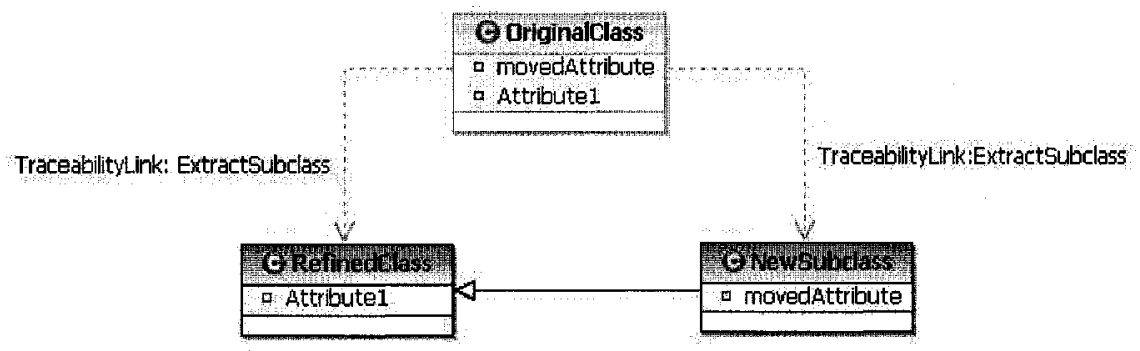

Figure 51 ExtractSubclass - Specification

User's Intention: Since a class has attributes and/or operations that are used only in some of its instances, it is necessary to create a subclass for that subset of attributes and/or operations.

Atomic Changes: Three or four kinds of atomic changes are used to derive the refinement: AddedClass, AddedGeneralization (between the added class and the class which the attribute/operation has been moved from), MovedAttribute and/or Movedoperation (to the added class). However, it is not required to have both MovedAttribute and Movedoperation at the same time since sometimes only some attributes are moved or only some operations are moved. Figure 52 (a) only presents the case that only one MovedAttribute is included in the group of atomic changes in order to make the OCL expressions more concise. 
Traceability Links: Two traceability links have to be established: one between the original class and the refined class; one between the original class and the new added class. These traceability links are specified in the OCL expressions in Figure 52 (b).

User Help: none.

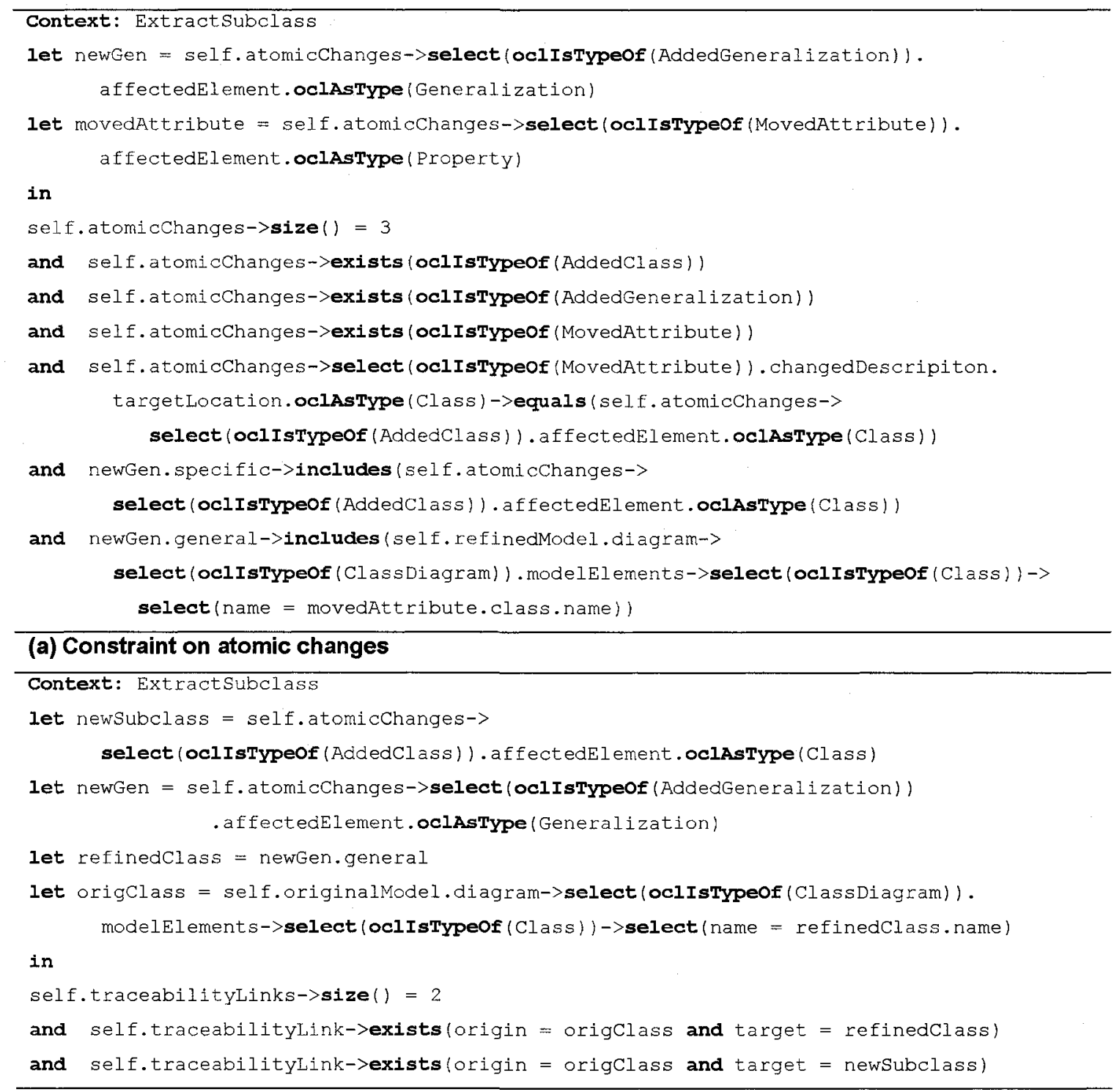

(b) Traceability links

Figure 52 Refinement Extractsubclass

Example: The example shown in Figure 53 is adapted from [20]. The original class Authorizationservice has four attributes and one operation. Since the attribute creditCard and the operation getcreditCard() are specific to the credit card 
authorization service, the new subclass CreditAuthorizationservice is added and used to model this specific authorization service.

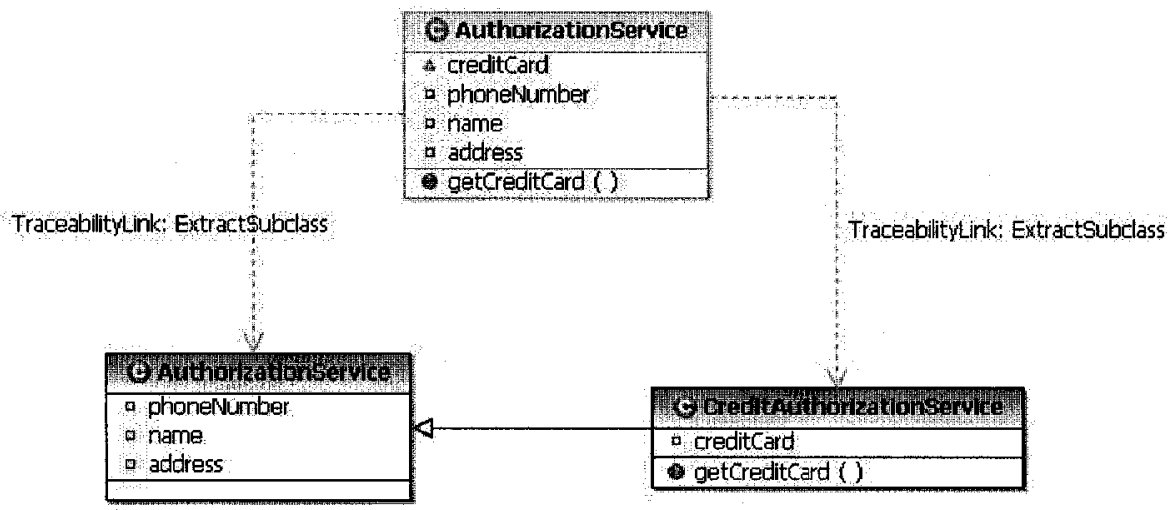

Figure 53 ExtractSubclass - Example 


\section{E.1.5 TurnAttriIntoClass}

Description: Transforms an attribute of the class being refined into a class of its own, which is then associated to the class being refined.

Specification: As shown in Figure 54, the attribute attribute1 of the class Originalclass is refined into the newly added class Attributel. The new association newAsso should be added to associate the class Attributel with the class RefinedClass.

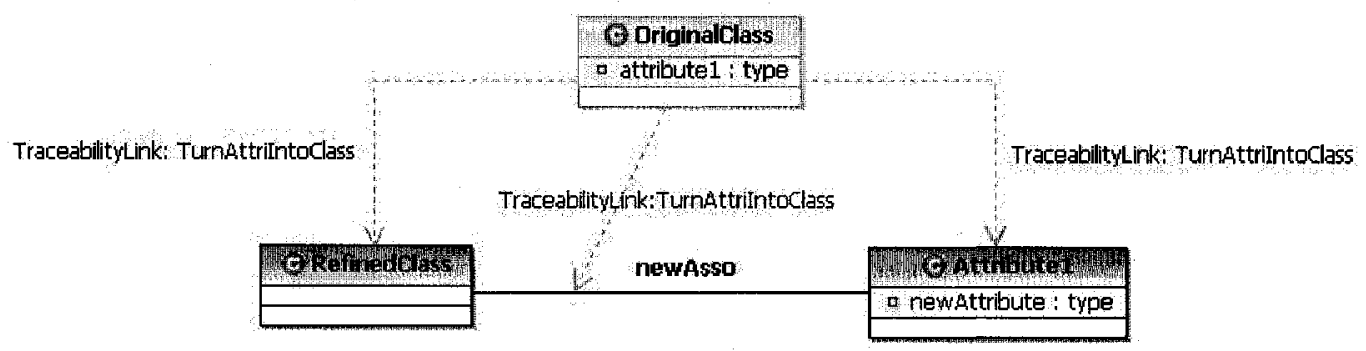

Figure 54 TurnAttriIntoclass - Specification

User's Intention: The attribute being refined contains too much information which has to be modeled using a class.

Atomic Changes: Three kinds of atomic changes are used to derive the refinement: AddedClass, AddedAssociation (between the added class and the class which the attribute is deleted from) and Deletedattribute. The specification of refinement TurnAttriIntoClass is shown as the OCL invariant for class TurnAttriIntoclass in Figure 55 (a).

Traceability Links: Three traceability links have to be established: one between the original class and the refined class; one between the original class and the new added class; one between the original class and the new added association. These traceability links are specified in the OCL expressions in Figure 55 (b). 
User Help: The user's help is required for identifying the refinement because it is very hard to guess that the deleted attribute is refined into the new added class based on the current resource we have.

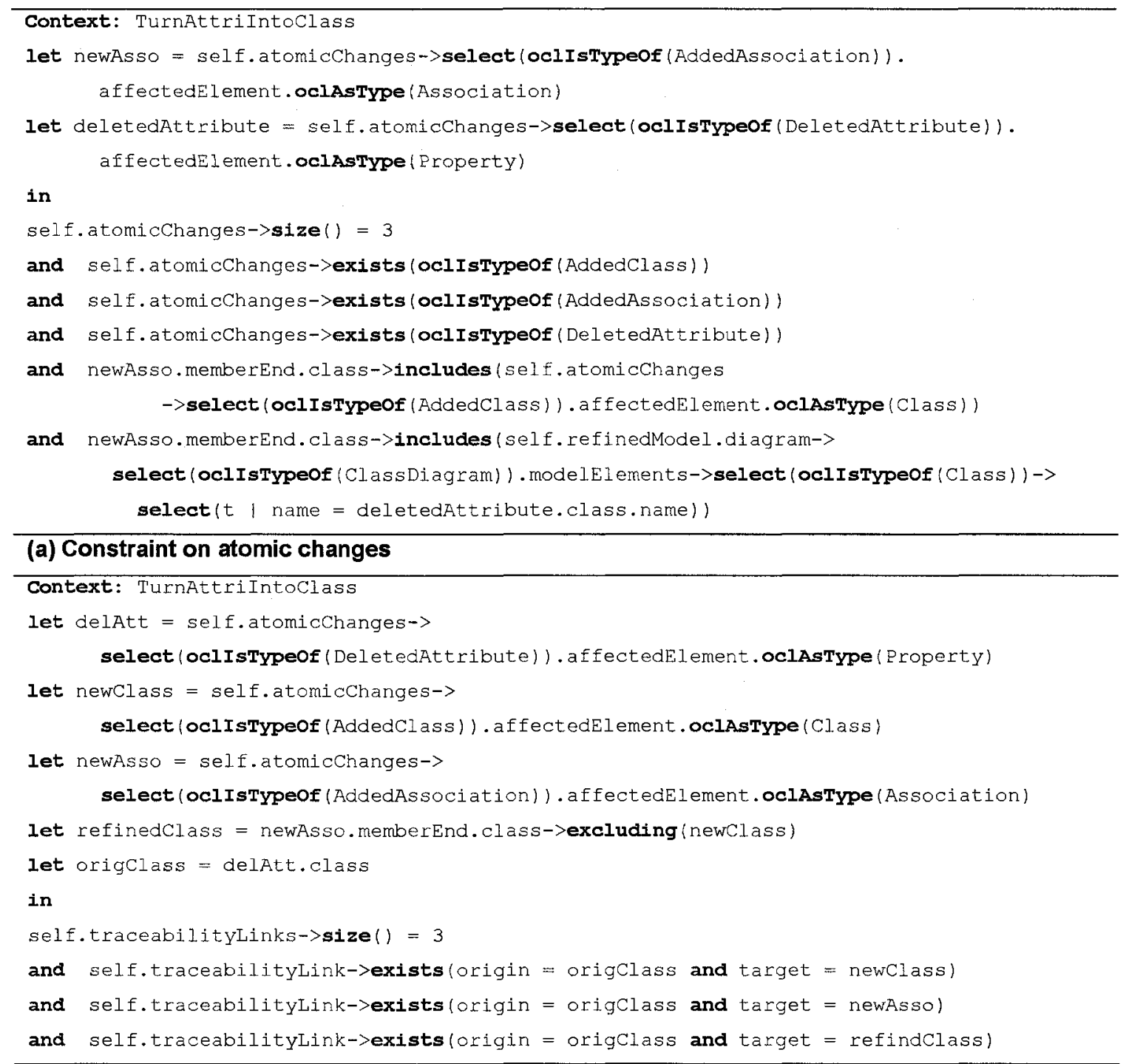

(b) Traceability links

Figure 55 Refinement TurnAttriIntoclass

Examples: The attribute customer of the class order (Figure 56(a)) is refined into the class Customer and the association between the classes order and customer (Figure $56(b))$. 


Oorder
+ customer : String

(a) original class diagram

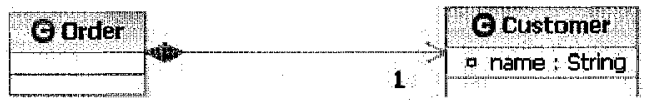

(b) refined class diagram

Figure 56 TurnAttriIntoclass - Example 


\section{E.1.6 TurnAttriIntoSubclass}

Description: Refines one attribute of the class being refined into its subclass.

Specification: As shown in Figure 57, the attribute attributel of the class Originalclass is refined into the newly added subclass Attribute1. The new generalization should be added between the classes Attributel and RefinedClass.

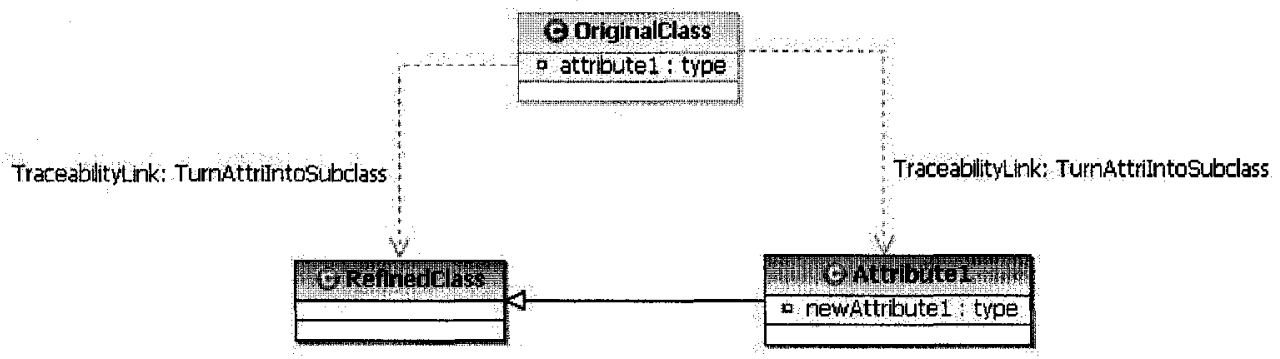

Figure 57 TurnAttriIntoSubclass - Specification

User's Intention: The attribute being refined contains too much information which has to be modeled using a class which can be the subclass of the class containing the deleted attribute originally.

Atomic Changes: Three kinds of atomic changes are used to derive the refinement: AddedClass, AddedGeneralization (between the added class and the class which the attribute has been deleted from) and DeletedAttribute. The specification of refinement TurnAttriIntosubclass is shown as the OCL invariant for class TurnAttriIntosubclass in Figure 58 (a).

Traceability Links: Two traceability links have to be established: one between the original class and the refined class and the new added class. These traceability links are specified in the OCL expressions in Figure 58 (b).

User Help: The user's help is required for identifying the refinement because it is very hard to guess that the deleted attribute is refined into the new added subclass based on the current resource we have. 


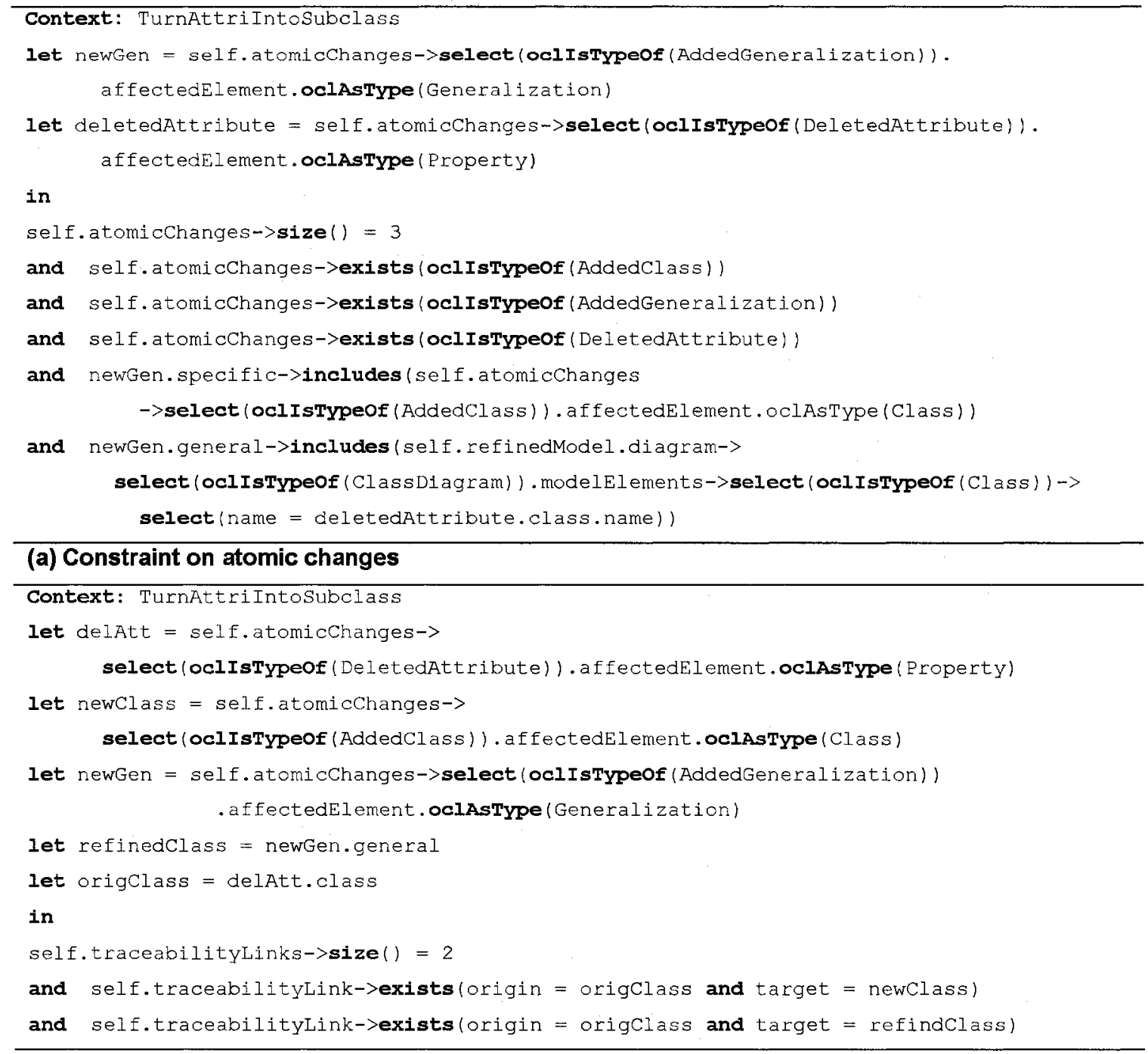

(b) Traceability links

Figure 58 Refinement TurnAttriIntoSubclass

Examples: Figure 59 provides an example. The class Employee of the original model (Figure 59 (a)) has two attributes: Eng ineer and Salesman. The attribute Engineer is refined into the subclass Engineer of the refined model (Figure 59 (b)). The attribute Salesman is refined into the subclass Salesman of the refined model (Figure 59 (b)). In this example, the refinement TurnAttriIntosubclass is applied twice. 


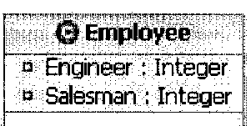

(a) original class diagram

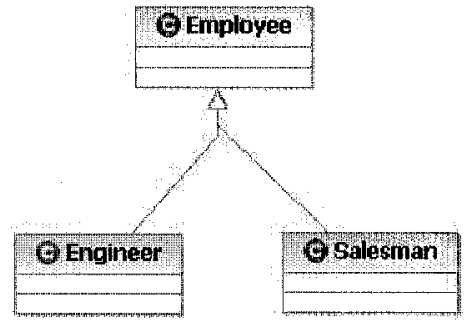

(b) refined class diagram

Figure 59 TurnAttriIntoclass - Example 


\section{E.1.7 TurnOprIntoRel}

Description: Refines one operation of the class being refined into a relationship connecting to it.

Specification: As shown in Figure 60, the operation Operation1() of the class Originalclass is refined into the newly added association newRel which is associated to the class Refinedclass which is refined from the class Originalclass .

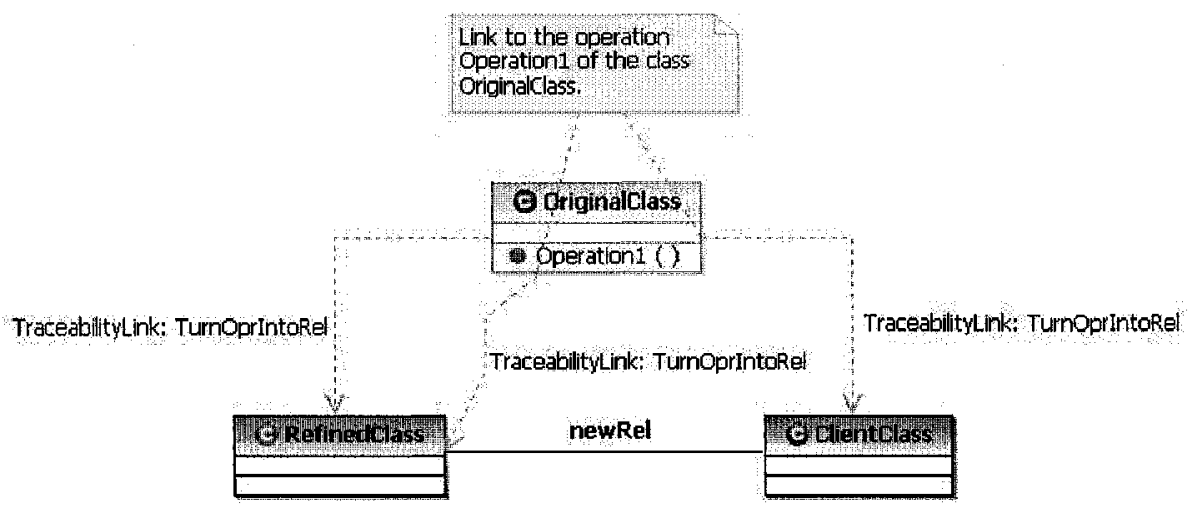

Figure 60 TurnoprIntoRel - Specification

User's Intention: The operation being refined can be more accurately modeled using an association instead.

Atomic Changes: Three kinds of atomic changes are used to derive the refinement: AddedAssociation, AddedClass, and Deletedoperation. The specification is shown as the OCL invariant for class TurnoprIntoRel in Figure 61 (a).

Traceability Links: Three traceability links have to be established: one between the original class and the refined class; one between the deleted operation and the new added association; one between the deleted operation and the added class. These traceability links are specified in the OCL expressions in Figure 61 (b). 
User Help: The user's help is required for identifying the refinement because it is very hard to guess that the deleted operation is refined into the new added association based on the current resource we have.

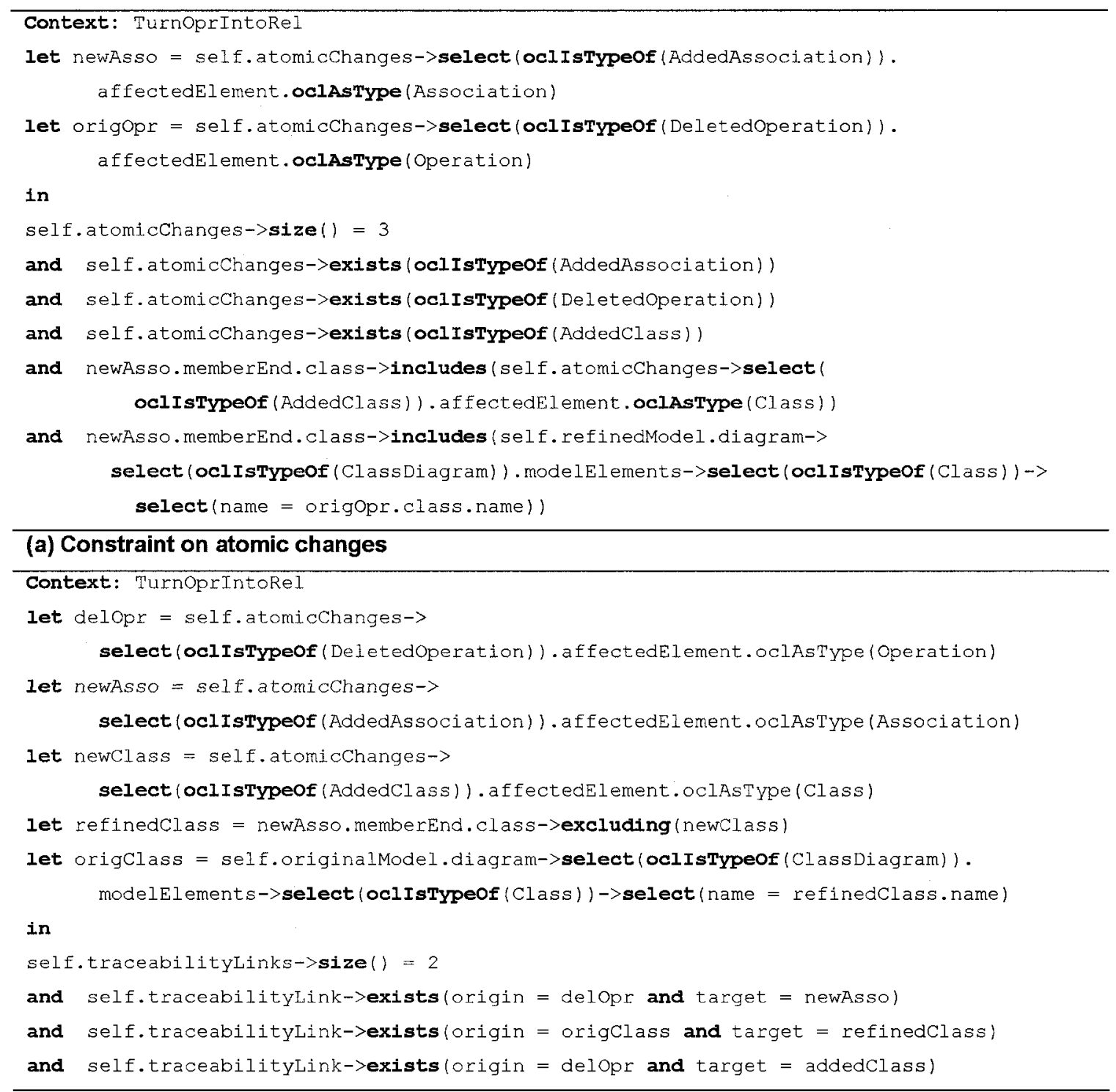

(b) Traceability links

Figure 61 Refinement TurnOpr IntoRel 


\section{E.2 Classes + Rels $\rightarrow$ Class}

The general purpose of this kind of refinement, where a group of classes and their relationships is refined into a single class, is to eliminate useless classes and relationships. Figure 62 provides a refined classification involving five types of refinements.

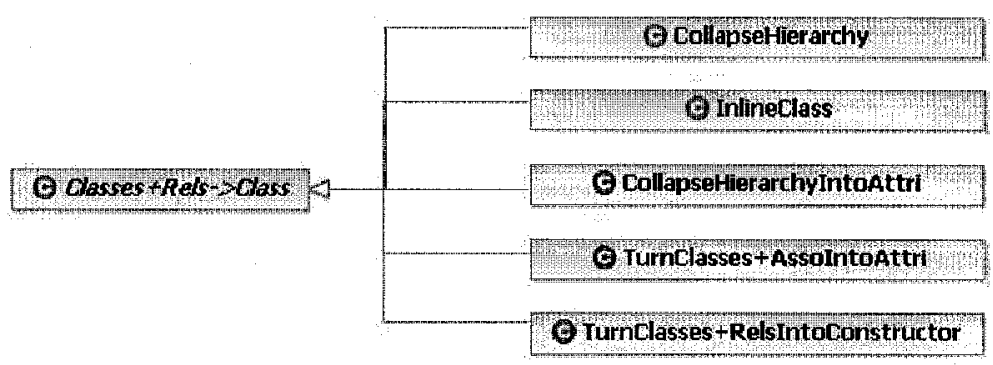

Figure 62 Taxonomy for Refinements of Type Classes+Rels->Class

We identify three sub-categories. A first type of purpose is that two classes are not very different and are thus merged. CollapseHierarchy (Section E.2.1) and Inlineclass (Section E.2.2) fall into this group: CollapseHierarchy merges a superclass and subclass together because they are not very different, and Inlineclass merges two classes together because one isn't doing very much. A second possible purpose is that it is not necessary to have classes and relationships to model what a single attribute can do. CollapseHierarchyIntoAttri (Section E.2.3) and Turnclasses+AssoIntoAttri (Section E.2.4) belong to this group: CollapseHierarchyIntoAttri refines several subclasses into an attribute of a superclass, and Turnclasses+AssoIntoAttri refines several classes and associations into an attribute of one of these classes. A third possible rationale is that a constructor is used to model what was originally modeled by several classes and relationships. Turnclasses+RelsIntoconstructor belongs to this group (Section E.2.5). 


\section{E.2.1 CollapseHierarchy}

Description: Merges a superclass and its subclass together because they are not very different.

Specification: As shown in Figure 63, the classes Originalsuperclass and Originalsubclass of the original model are merged into the class Singleclass of the refined model.

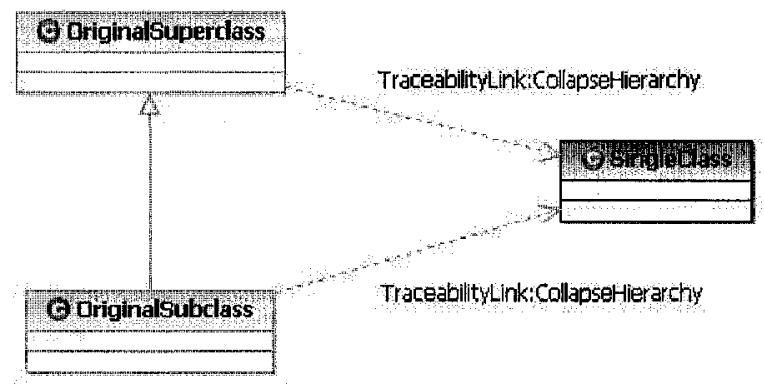

Figure 63 CollapseHierarchy - Specification

User's Intention: See description.

Atomic Changes: Three kinds of atomic changes are used to derive the refinement: DeletedClass, DeletedGeneralization (which links the deleted class) and MovedAttribute (to the undeleted class involved in the generalization). If the class Originalsuperclass is deleted, some or all of its attributes and/or operations are moved to the class Originalsubclass. Otherwise, if the class Originalsubclass is deleted, some or all of its attributes and/or operations are moved to the class Originalsuperclass. Figure 64 (a) only presents the latter case with only one moved attribute.

Traceability Links: Two traceability links have to be established: one between the classes Originalsuperclass (referred to as origsuperclass) and Singleclass (referred to as singleclass); one between the classes Originalsubclass (referred to as origSubclass) and Singleclass. These traceability links are specified in the OCL expressions using Figure 64 (b). 
User Help: none.

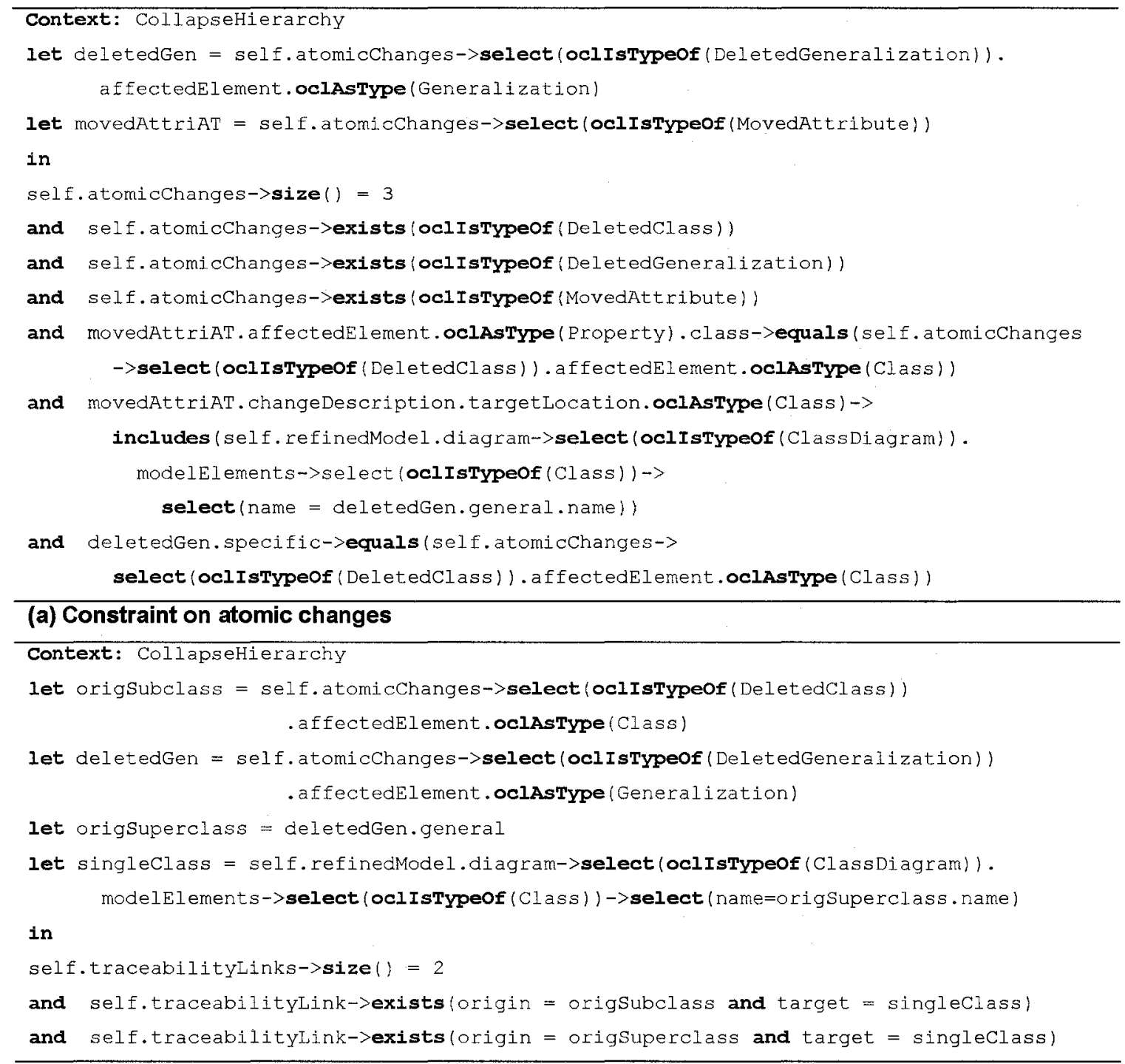

(b) Traceability links

Figure 64 Refinement CollapseHierchary 


\section{E.2.2 InlineClass}

Description: Merges two associated classes together because one of them isn't doing very much.

Specification: As shown in Figure 65, the classes Originalclass 1 and Originalclass 2 of the original model are merged into the class Singleclass of the refined model.

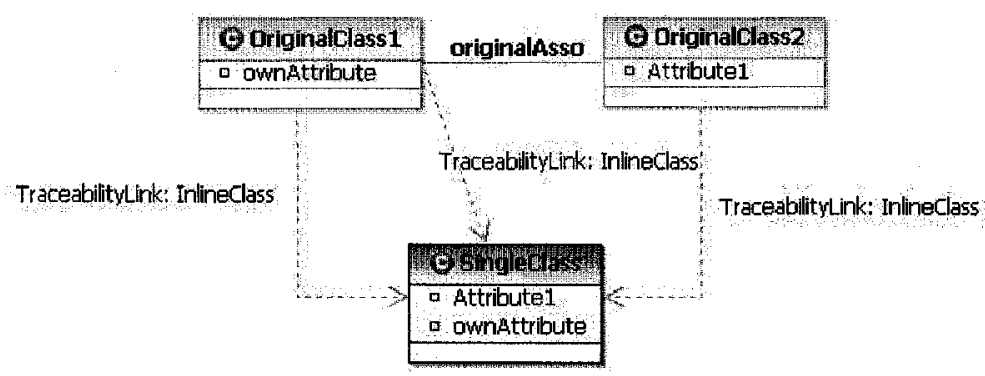

Figure 65 Inlineclass - Specification

User's Intention: See description.

Atomic Changes: Three kinds of atomic changes are used to derive the refinement: DeletedClass, DeletedAssociation (involving the deleted class) and MovedAttribute (from the deleted class to the other class involved in the deleted association). The specification is shown as the OCL invariant for class Inlineclass in Figure 66 (a). We assume that the class Originalclass 2 is deleted and one of attributes of the class Originalclass 2 is moved to the class Originalclass 1 even though there are situations in which more than one or all attributes and/or operations are moved.

Traceability Links: Three traceability links have to be established: one between the classes Originalclass1 (referred to as origClass1) and Singleclass (referred to as singleclass); one between the classes Originalclass2 (referred to as origsubclass2) and Singleclass; one between the association originalAsso (referred to as origAsso) and the class singleclass. These traceability links are specified using the OCL expressions in Figure 66 (b). 
User Help: none.

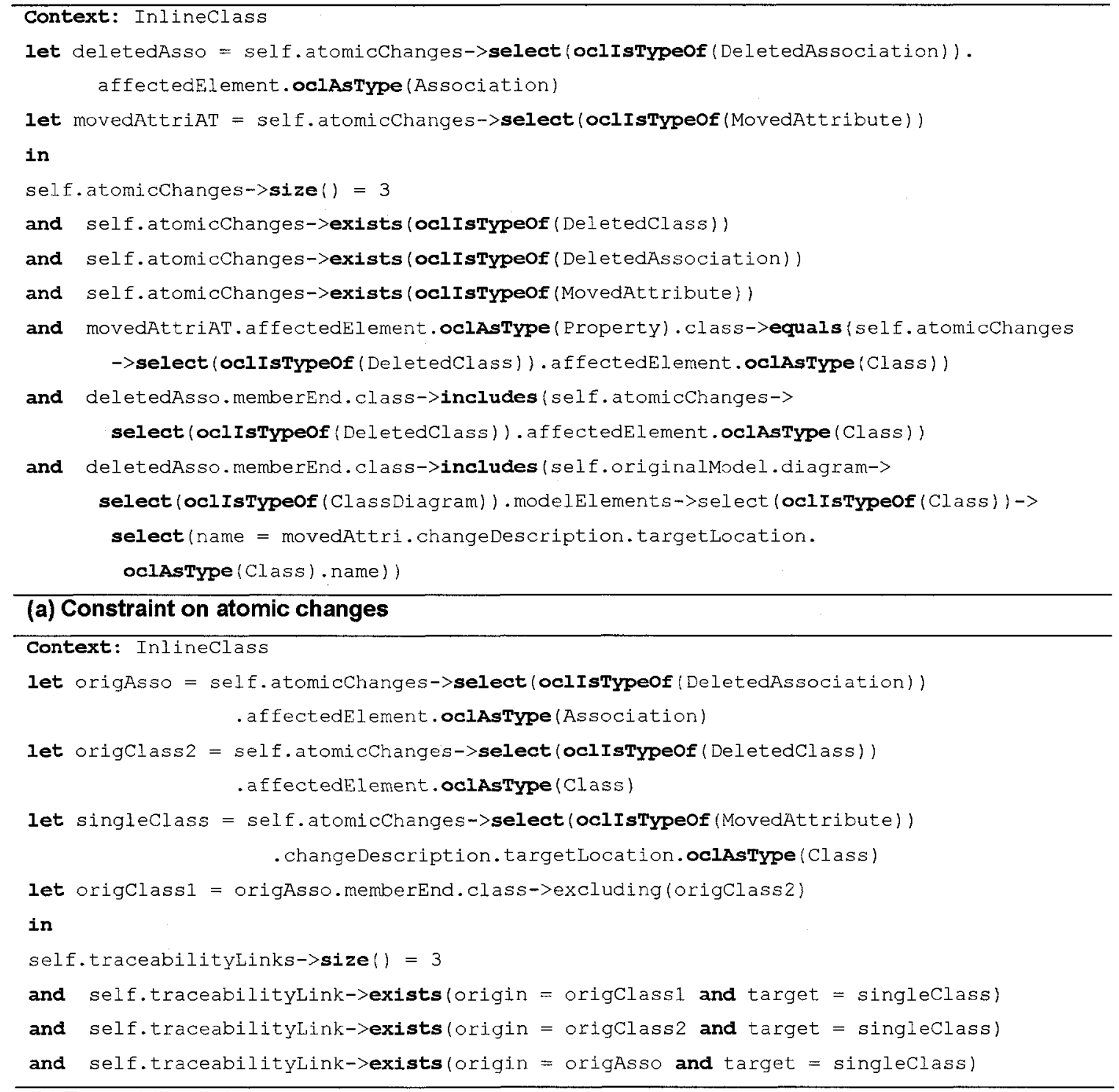

(b) Traceability links

\section{Figure 66 Refinement Inlineclass}

Example: Figure 67 provides an example. The classes Person and Telephone Number are connected by the association officeTelephone in the original class diagram (Figure 67 (a)). Since the class Telephone Number is not doing very much, we can move all attributes of the class Telephone Number into the class Person and delete the class Telephone Number (Figure 67 (b)). 


\begin{tabular}{|c|c|c|}
\hline OPerson & \multirow{2}{*}{ officeTelephone } & c telephorie Number \\
\hline \multirow{3}{*}{$\begin{array}{l}\text { o name } \\
\text { getTelephoneNumber () }\end{array}$} & & a areacode \\
\hline & & 0 number \\
\hline & & 9 get TelephoneNumber (1) \\
\hline
\end{tabular}

(a) original class diagram

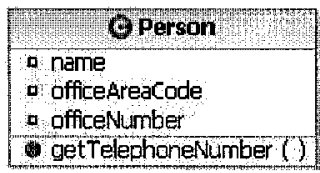

(b) refined class diagram

Figure 67 Inlineclass - Example 


\section{E.2.3 CollapseHierarchyIntoAttri}

Description: Refines several subclasses of a superclass into one of the attributes of the superclass.

Specification: As shown in Figure 68, the subclasses Subclass 1 and subclass 2 are refined into the attribute newAttribute of the class RefinedClass which is refined from the class Originalsuperclass.

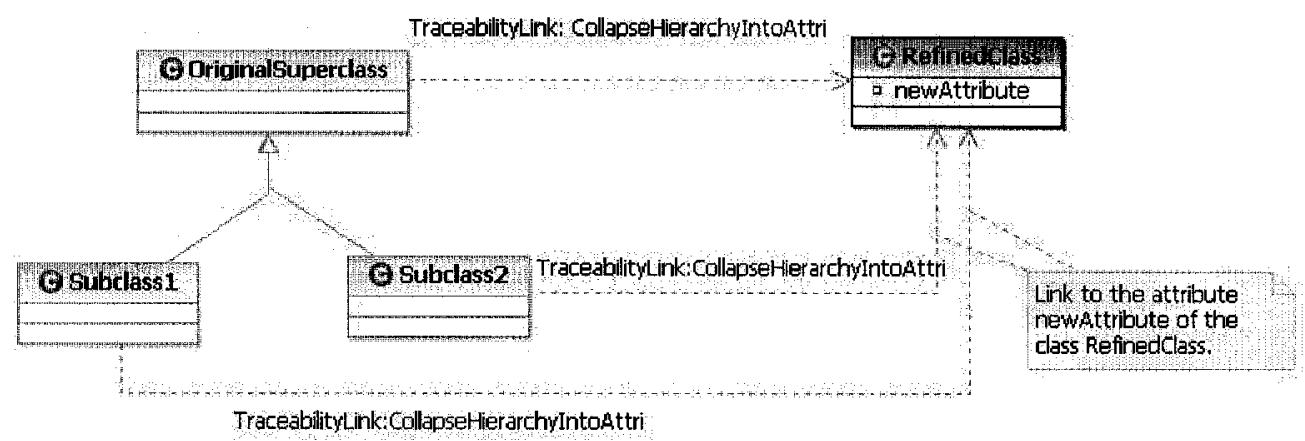

Figure 68 CollapseHierarchyIntoAttri - Specification

User's Intention: An attribute can be used to model what the two subclasses do. For example, these two subclasses have no their own attributes or operations.

Atomic Changes: Three kinds of atomic changes are used to derive the refinement: DeletedClass, DeletedGeneralization (linking the deleted class and the class receiving the added attribute) and AddedAttribute. The specification is shown as the OCL invariant for class CollapseHierarchyIntoAttri in Figure 69 (a). Note that the atomic changes DeletedClass and DeletedGeneralization may appear twice or more. In the OCL expressions in Figure 69, we assume that these two atomic changes perform twice only in order to make the OCL expression more concise.

Traceability Links: Three traceability links have to be established: one between the classes Originalsuperclass (referred to as origsuperclass) and RefinedClass (referred to as refinedClass); one between the class Subclassl (referred to as origSubclass1) and the attribute newAttribute (referred to as newAttri) of the class 
RefinedClass; one between the class Subclass2 (referred to as origSubclass2) and the attribute newAttribute of the class RefinedClass. These traceability links are specified in the OCL expressions in Figure 69 (b).

User Help: the user's help is required, since we can not distinguish the user's intent of adding a new attribute to the superclass.

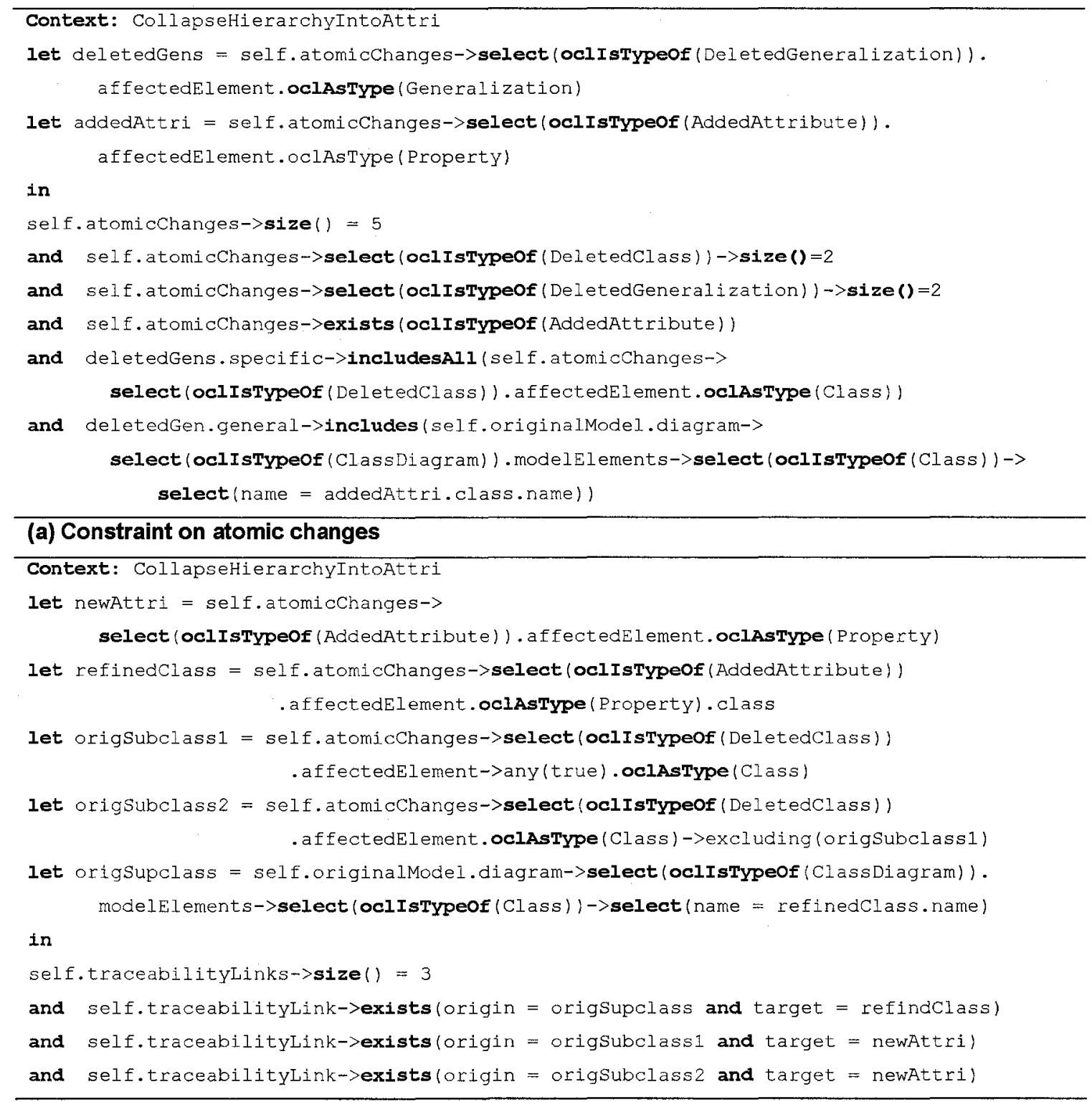

(b) Traceability links

Figure 69 Refinement CollapseHierarchyIntoAttri 


\section{E.2.4 TurnClasses+AssoIntoAttri}

Description: Refines several classes and associations into an attribute of one of these classes.

Specification: As shown in Figure 70, the class Originalclass 2 and the association origAsso are refined into the attribute newAttribute of the class RefinedClass which is refined from the class Originalclass 1.

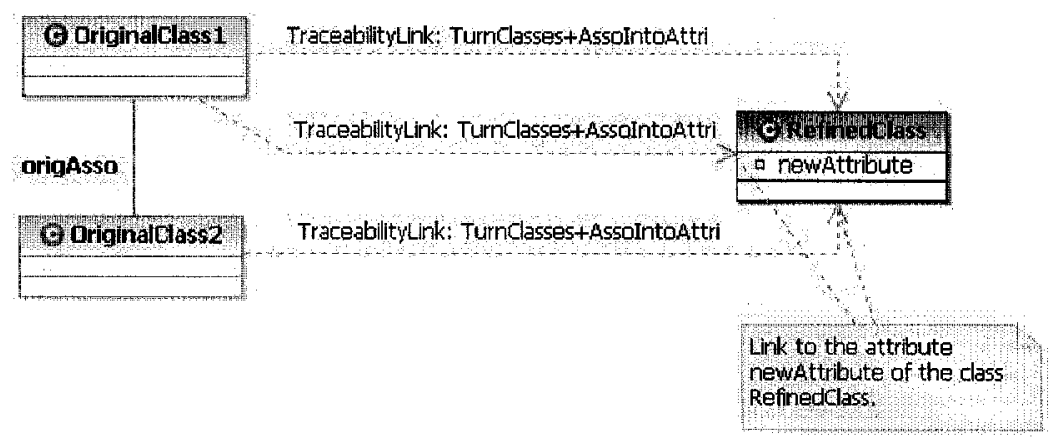

Figure 70 Turnclasses+AssoIntoAttri - Specification

User's Intention: An attribute can be used to model what several classes and associations do.

Atomic Changes: Three kinds of atomic changes are used to derive the refinement: DeletedClass, DeletedAssociation (between the deleted class and the class receiving the new attribute) and AddedAttribute. The specification is shown as the OCL invariant for class TurnClasses+AssoIntoAttri in Figure 71 (a). Note that the atomic changes DeletedClass and DeletedAssociation may appear once or more. In the OCL expressions in Figure 71, we assume that these two atomic changes appear once only in order to make the OCL expression more concise.

Traceability Links: Three traceability links have to be established: one between the classes Originalclassl (referred to as origClass1) and RefinedClass (referred to as refinedClass); one between the class Originalclass2 (referred to as origclass2) and the attribute newAttribute (referred to as newAttri) of the class RefinedClass; 
one between the association origAsso (referred to as origAsso) and the attribute newAttribute of the class RefinedClass. These traceability links are specified in the OCL expressions in Figure 71 (b).

User Help: The user's help is required, since we can not distinguish the user's intent of adding a new attribute to the superclass.

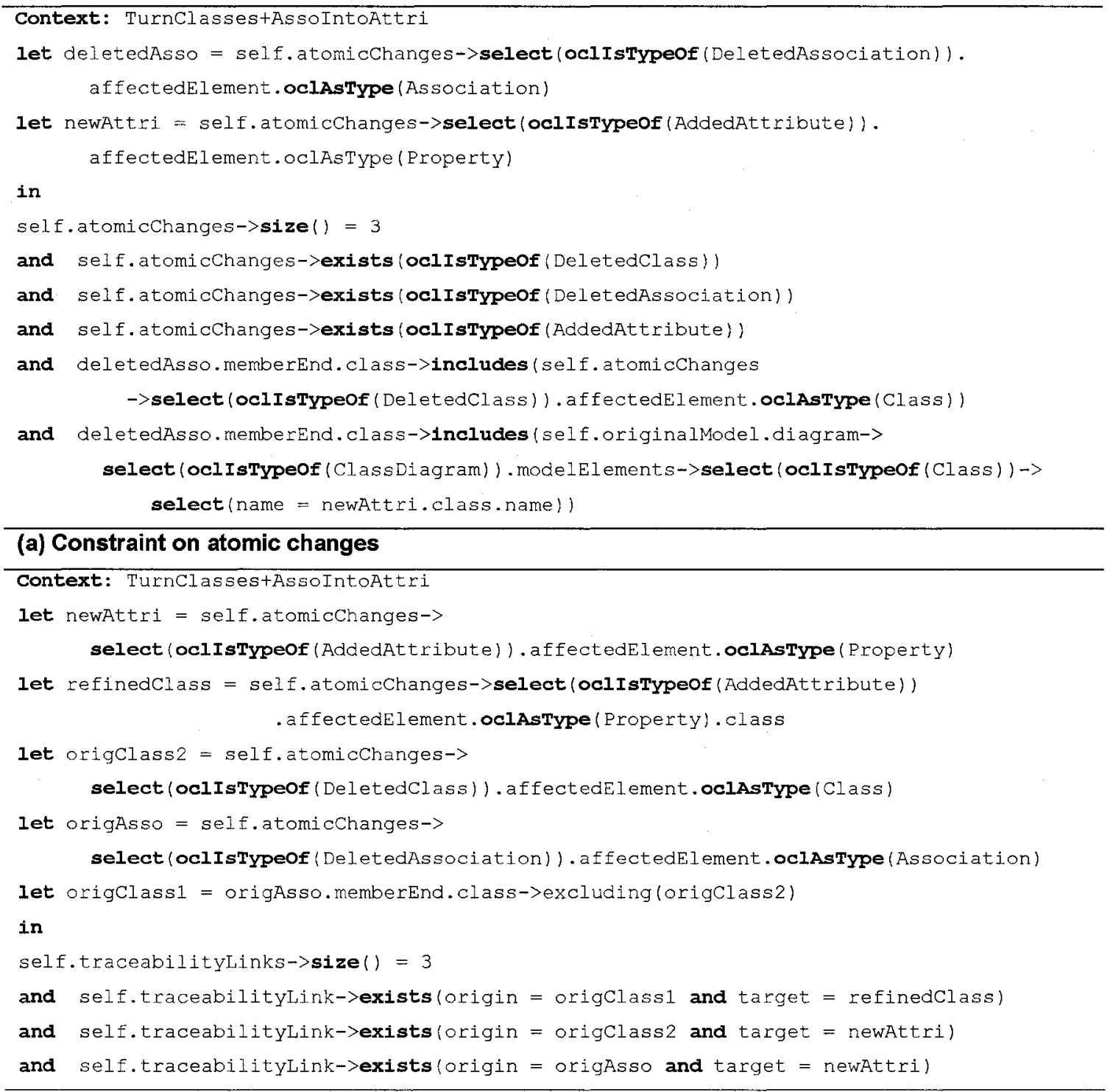

(b) Traceability links

Figure 71 Refinement TurnClasses+AssoIntoAttri 


\section{E.2.5 TurnClasses+RelsIntoConstructor}

Descripiton: Refines several classes and relationships into a constructor of one of these classes.

Specification: As shown in Figure 72, the class Originalclass 2 and the association origAsso are refined into the constructor RefinedClass() of the class RefinedClass which is refined from the class Originalclass 1.

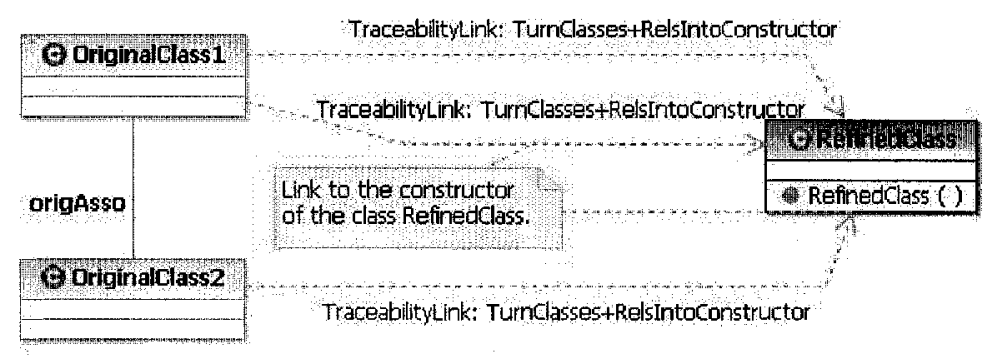

Figure 72 Turnclasses+Rels IntoConstructor - Specification

User's Intention: The constructor of a class can be used to model what the class and the association do.

Atomic Changes: Three kinds of atomic changes are used to derive the refinement: DeletedClass, DeletedAssociation (between the deleted class and the class receiving the new constructor) and Addedoperation. The specification is shown as the OCL invariant for class TurnClasses+AssoIntoConstructor in Figure 73 (a). Note that the atomic changes DeletedClass and DeletedAssociation may appear once or more. In the OCL expressions in Figure 73, we assume that these two atomic changes appear once only in order to make the OCL expression more concise.

Traceability Links: Three traceability links have to be established: one between the classes Originalclass 1 (referred to as origClass1) and RefinedClass (referred to as refinedClass); one between the class Originalclass2 (referred to as origclass2) and the constructor RefinedClass() (referred to as newConstructor) of the class RefinedClass; one between the association origAsso (referred to as origAsso) and 
the constructor RefinedClass() of the class RefinedClass. These traceability links are specified in the OCL expressions in Figure 73 (b).

User Help: The user's help is required, since we can not distinguish the user's intent of adding a new constructor for the class being refined.

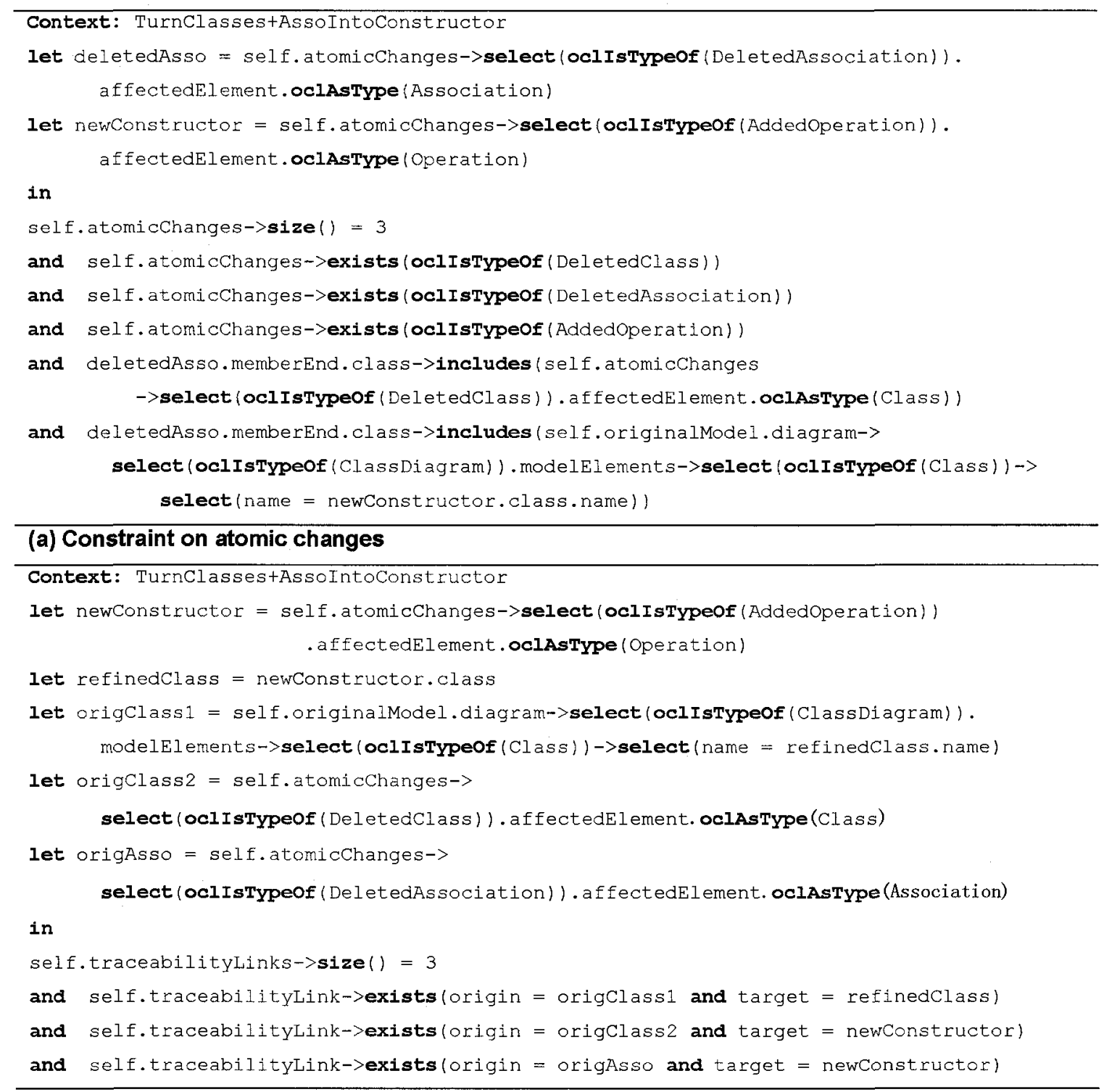

(b) Traceability links

Figure 73 Refinement TurnClasses+AssoIntoConstructor 


\section{E.3 Classes+Rels $\rightarrow$ Classes + Rels}

This refinement refines a class diagram in order to improve its efficiency and reusability. It can be very complex and it may affect numerous classes and relationships. Figure 74 provides a refined classification involving seven types of refinements. PullUpAttribute (Section E.3.1), PullUpoperation (Section E.3.2), PushDownAttribute (Section E.3.3), and PushDownoperation (Section E.3.4) are refinements operating on attributes and operations of two classes connecting by generalization relationship. EliminateDuplibyInheritance (Section E.3.5), EliminateDupliByComposition (Section E.3.6) and splitsubclass (Section E.3.7) are refinements simply changing the structure of the diagram.

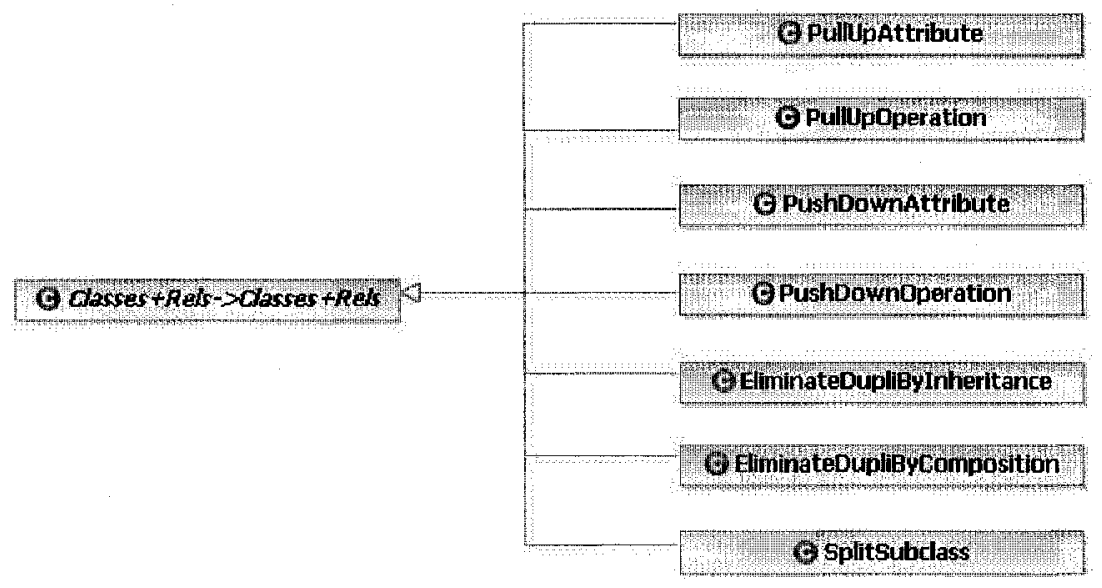

Figure 74 Taxonomy for Refinements of Type Classes+Rels->Classes+Rels 


\section{E.3.1 PullUpAttribute}

Description: Pulls up one attribute common to all subclasses of a superclass to the superclass.

Specification: As shown in Figure 75, the common attribute CommonAttribute of the classes Subclass1 and Subclass2 is moved into the superclass RefinedsuperClass which is refined from the class superclass.

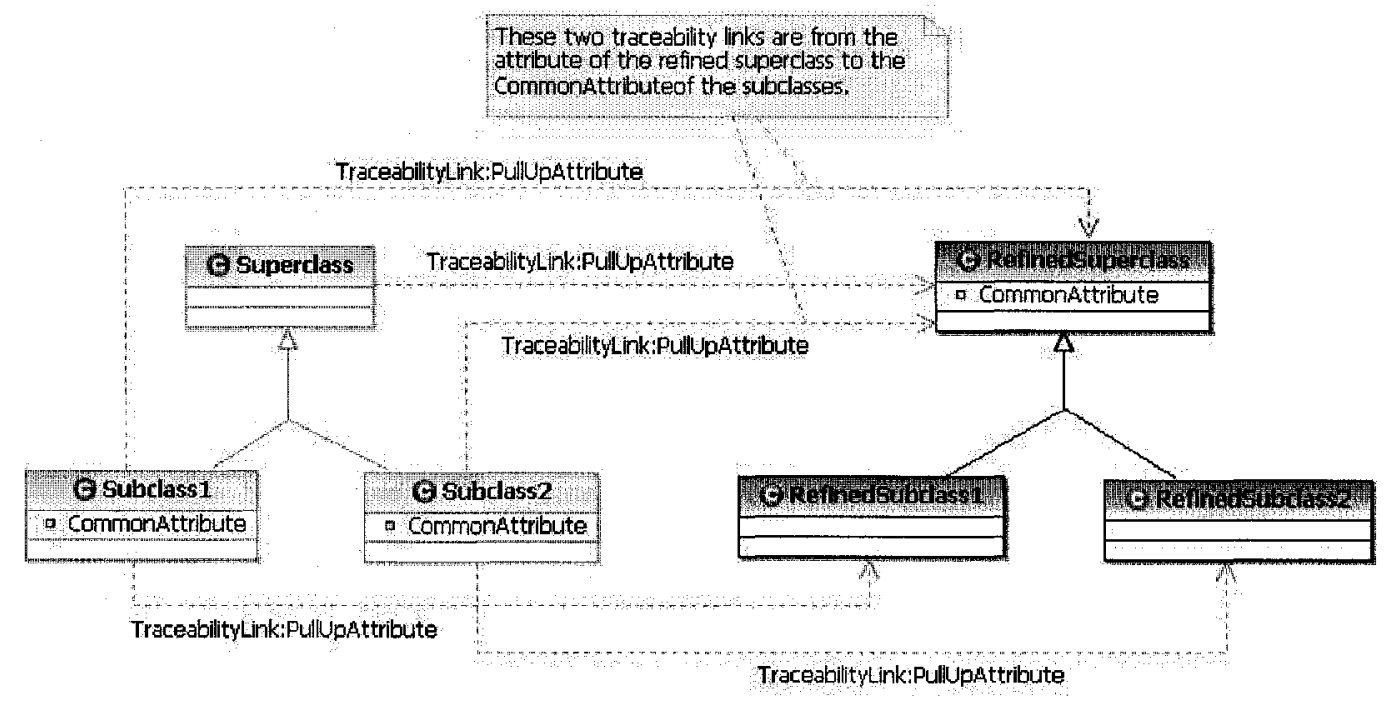

Figure 75 PullUpAttribute - Specification

User's Intention: Duplicate attributes exist in subclasses. By pulling up these attributes into the superclass, duplication can be reduced.

Atomic Changes: Two kinds of atomic changes are used to derive the refinement: MovedAttribute and DeletedAttribute. The specification is shown as the OCL invariant for class PullupAttribute in Figure 76 (a). Note that the atomic changes DeletedAttribute may appear more than once because it is possible to pull up the common attribute from more than two subclasses. In the OCL expressions in Figure 76, we assume that these two atomic changes appear once only, the attribute CommonAttribute of the class subclass1 is moved into the superclass, and the 
CommonAttribute of the class Subclass 2 is deleted, in order to make the OCL expression more concise.

Traceability Links: Five traceability links have to be established (Figure 76 (b)):

- one between the classes superclass (referred to as origsup) and Refinedsuperclass (referred to as refinedsup);

- one between the classes Subclassi (referred to as origsub) and RefinedSubclass 1 (referred to as refinedsub1);

- one between the classes subclass2 (referred to as origsub2) and RefinedSubclass2 (referred to as refinedSub2);

- one between the attribute CommonAttribute (referred to as comAttrioforigsub1) of the class Subclass1 and the attribute CommonAttribute (referred to as comAttriRefinedsup) of the class RefinedSuperclass;

- one between the attribute commonAttribute(referred to as comAttrioforigsub2) of the class subclass2 and the attribute CommonAttribute of the class RefinedSuperclass.

These traceability links are specified in the OCL expressions in Figure 76 (b).

User Help: none. 


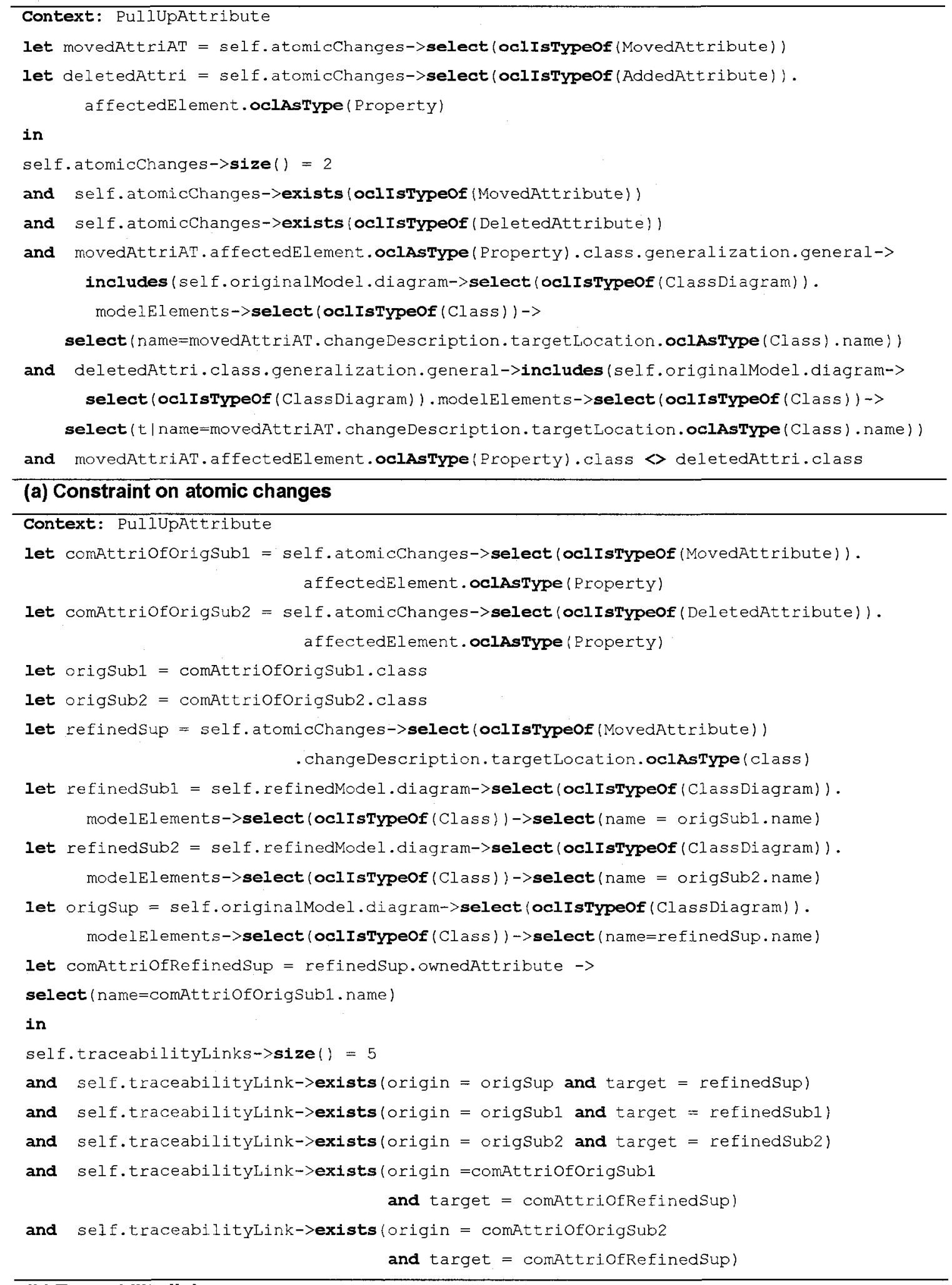

(b) Traceability links

Figure 76 Refinement PullUpAttribute 


\section{E.3.2 PullUpOperation}

Description: Pulls up one operation common to all subclasses of a superclass to the superclass.

Specification: As shown in Figure 77, the common operation Commonoperation() of the classes subclass 1 and subclass2 is moved into the superclass RefinedSuperclass which is refined from the class superclass.

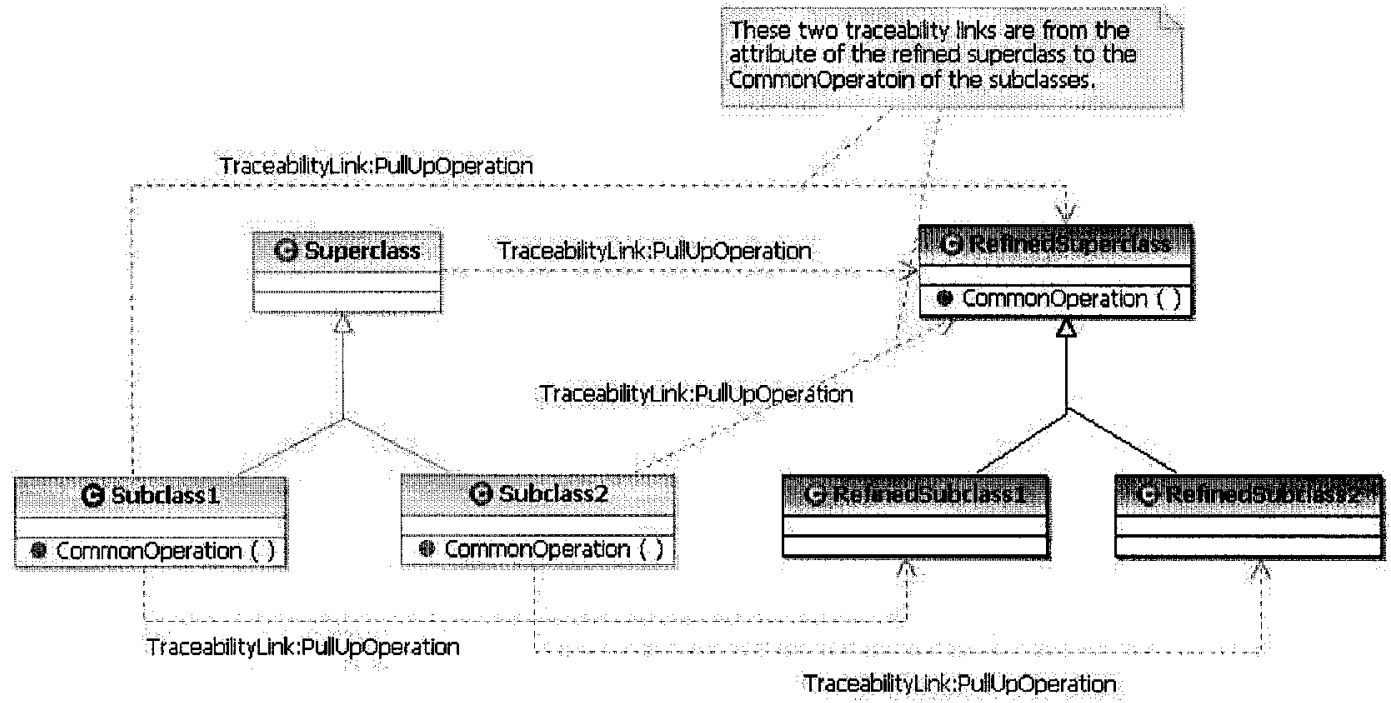

Figure 77 PullUpoperation - Specification

User's Intention: Duplicate operations exist in subclasses. By pulling up these operations into the superclass, duplication can be reduced.

Atomic Changes: Two kinds of atomic changes are used to derive the refinement: Movedoperation and Deletedoperation. The specification is shown as the OCL invariant for class Pullupoperation in Figure 78 (a). Note that the atomic change Deletedoperation may appear more than once because it is possible to pull up the common operation from more than two subclasses. In the OCL expressions in Figure 78, we assume that these two atomic changes appear once only, the operation Commonoperation() of the class Subclassl is moved into the superclass, and the 
Commonoperation() of the class Subclass 2 is deleted, in order to make the OCL expression more concise.

Traceability Links: Five traceability links have to be established:

- one between the classes superclass (referred to as origsup) and RefinedSuperclass (referred to as refinedSup);

- one between the classes Subclass1 (referred to as refinedorigsub) and RefinedSubclass1 (referred to as refinedsub1);

- one between the classes Subclass2 (referred to as origrefinedSub2) and RefinedSubclass2 (referred to as refinedSub2);

- one between the attribute Commonoperation() (referred to as comoproforigsub1) of the class subclass1 and the attribute Commonoperation() (referred to as comoprofRefinedsup) of the class Refinedsuperclass;

- one between the attribute Commonoperation() (referred to as comoproforigsub2) of the class subclass2 and the attribute Commonoperation() of the class RefinedSuperclass.

These traceability links are specified in the OCL expressions in Figure 78 (b).

\section{User Help: none.}




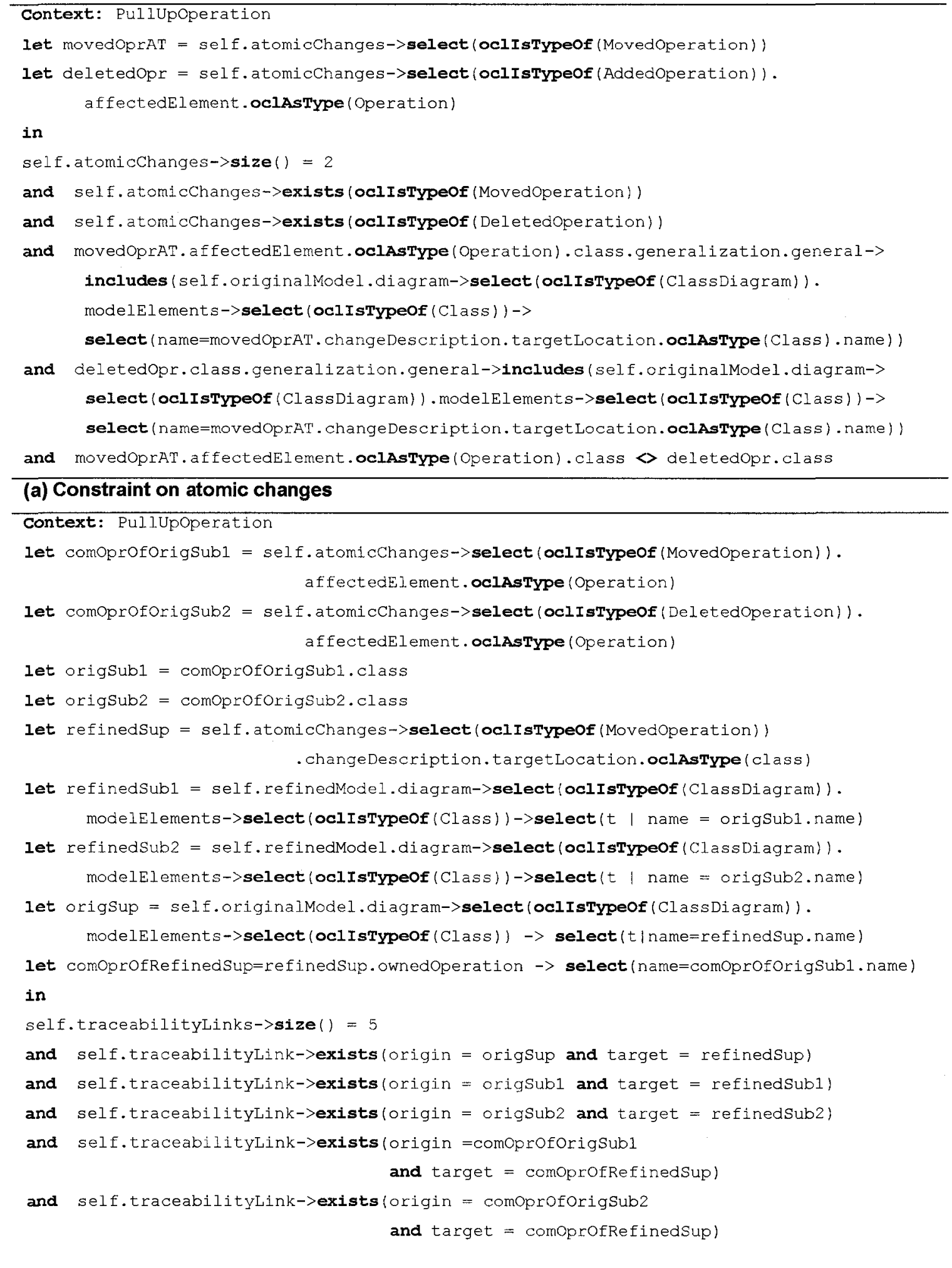

(b) Traceability links

Figure 78 Refinement Pullupoperation 


\section{E.3.3 PushDownAttribute}

Description: Pushs down one of the attributes of a superclass to one of its subclasses, because this attribute is used only by this subclass.

Specification: As shown in Figure 79, the common attribute Attribute of the superclass Superclass is moved into the subclass RefinedSubclass which is refined from the class Subclass.

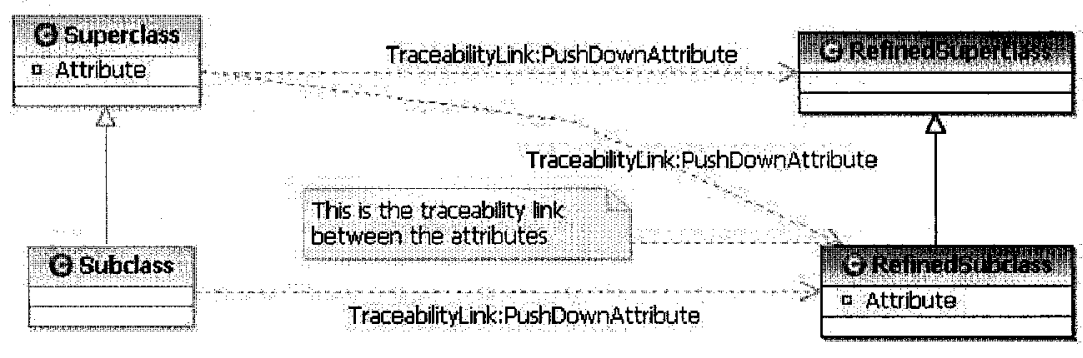

Figure 79 PushDownAttribute - Specification

User's Intention: This refinement is the opposite of PullupAttribute. Because the attributes are not used in the superclass anymore but only in a subclass, the attributes can be pushed down to the subclass.

Atomic Changes: One kind of atomic change is used to derive the refinement: MovedAttribute. The specification is shown as the OCL invariant for class PushDownwAttribute in Figure 80 (a).

Traceability Links: Three traceability links have to be established: one between the classes Superclass (referred to as origsup) and Refinedsuperclass (referred to as refinedSup); one between the classes Subclass (referred to as origsub) and RefinedSubclass (referred to as refinedSub); one between the attribute Attribute (referred to as attrioforigsup) of the class superclass and the attribute Attribute (referred to as attriofRefinedSup) of the class RefinedSubclass. These traceability links are specified in the OCL expressions in Figure 80 (b).

\section{User Help: none.}




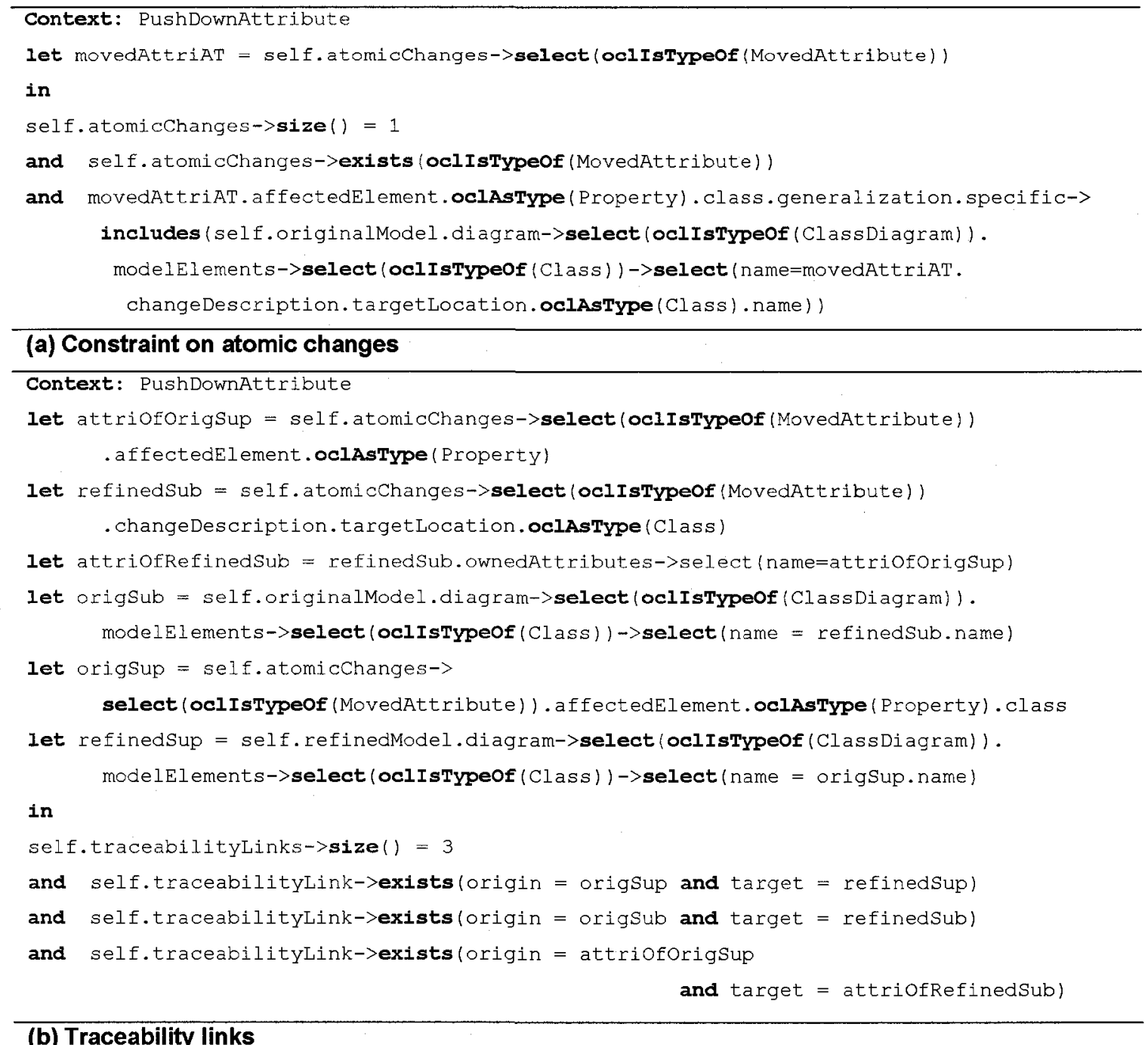

Figure 80 Refinement PushDownAttribute 


\section{E.3.4 PushDownOperation}

Descripsion: Pushs down one of the operations of a superclass to one of its subclasses, because this operation is used only by this subclass.

Specification: As shown in Figure 81, the common operation operation() of the superclass Superclass is moved into the subclass RefinedSubclass which is refined from the class Subclass.

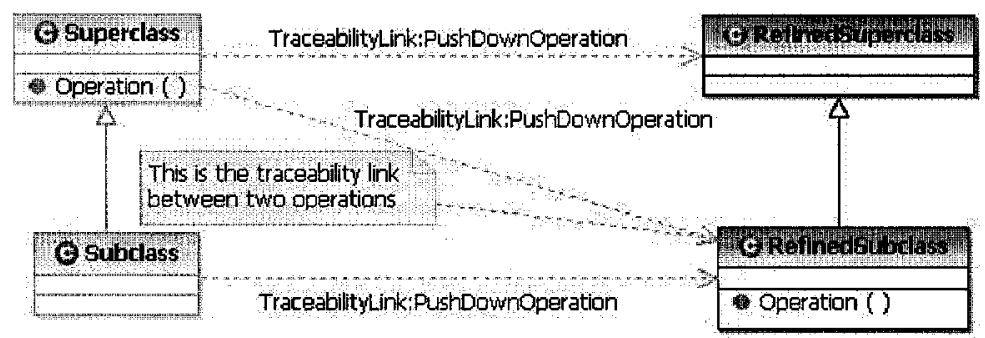

Figure 81 PushDownoperation - Specification

User's Intention: This refinement is the opposite of Pullupoperation. Because the operatoins are not used in the superclass anymore but only in subclass, the operations can be pushed down to the subclass.

Atomic Changes: One kind of atomic change is used to derive the refinement: Movedoperation. The specification is shown as the OCL invariant for class PushDownoperation in Figure 82 (a).

Traceability Links: Three traceability links have to be established: one between the classes Superclass (referred to as origSup) and RefinedSuperclass (referred to as refinedSup); one between the classes subclass (referred to as origsub) and RefinedSubclass (referred to as refinedSub); one between the operation opertion() (referred to as oproforigsup) of the class Superclass and the operation operation() (referred to as oprofRefinedsup) of the class RefinedSubclass. These traceability links are specified in the OCL expressions in Figure 82 (b). 
User Help: none.

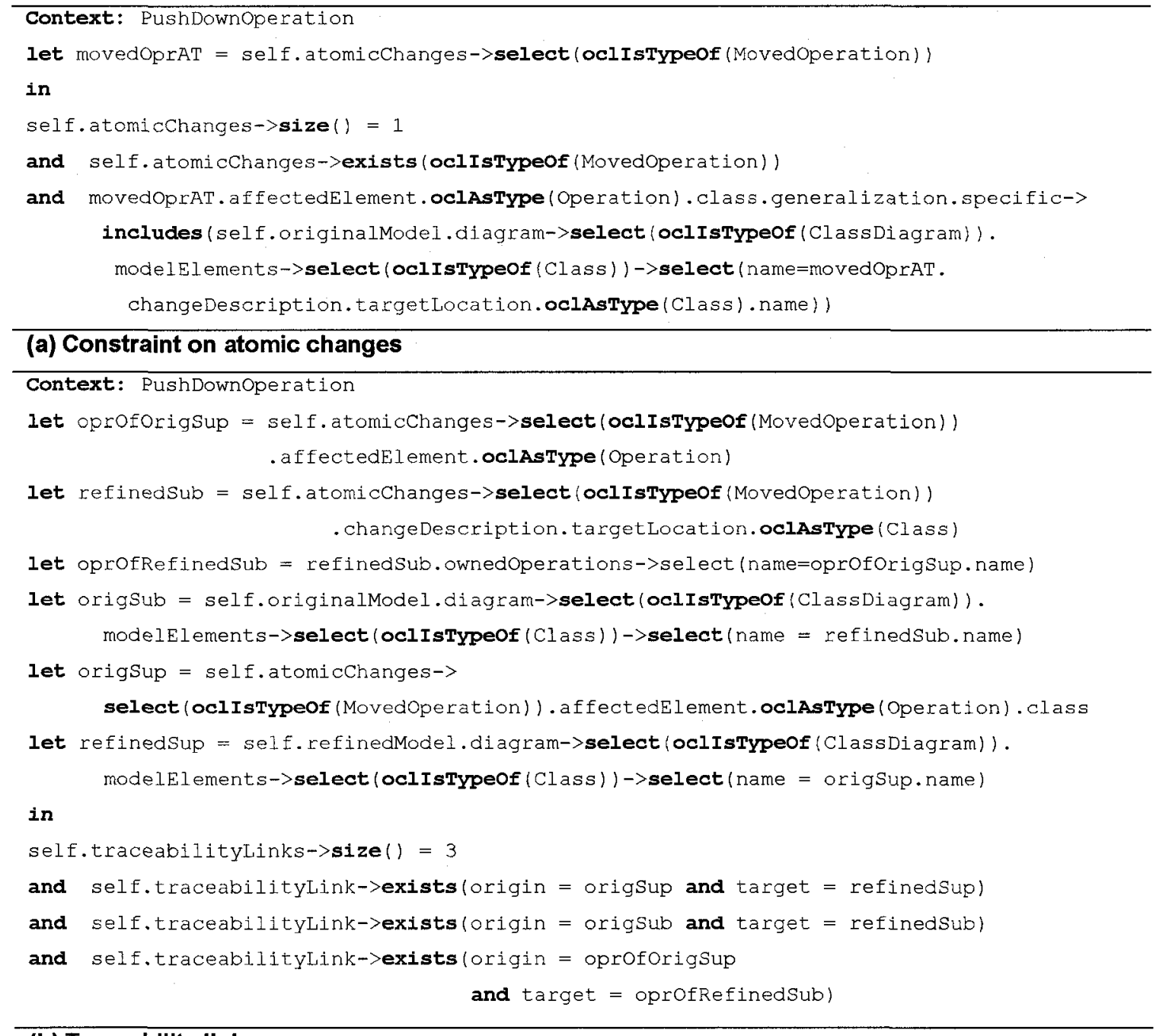

(b) Traceability links

Figure 82 Refinement PushDownoperation 


\section{E.3.5 EliminateDupliByInheritance}

Description: Extracts out the common attributes and operations of several classes, and create a superclass for these classes to store these common attributes and operations.

Specification: As shown in Figure 83, the attribute CommonAttribute and the operation Commonoperation() of the classes Originalclass1 and Originalclass2 are extracted out and put into the newly added superclass superclass. The class RefinedClass1 is refined from the class originalclass1, and the class RefinedClass 2 is refined from the class Originalclass2.

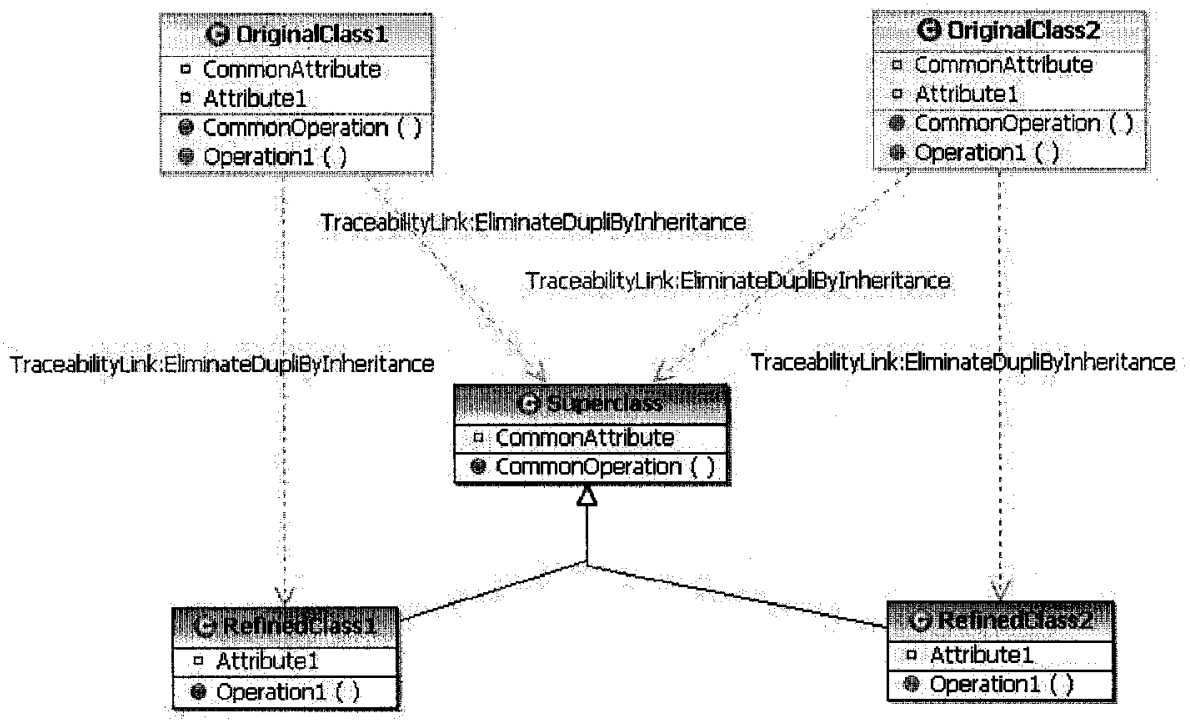

Figure 83 EliminateDupliByInheritance - Specification

User's Intention: Because two classes have similar attribute(s) and/or operation(s), a superclass can be created to contain the similar attribute(s) and/or operation(s).

Atomic Changes: Six kinds of atomic changes are used to derive the refinement: MovedAttribute, Movedoperation, AddedClass, AddedGeneralization, DeletedAttribute, and Deletedoperation. The specification is shown as the OCL invariant for class EliminateDupliByInheritance in Figure 84 (a). Note that the atomic changes DeletedAttribute and Deletedoperation may appear more than once because it is possible to extract out the common attribute and operation from more 
than two classes. In the OCL expressions in Figure 84 (a) and Figure 85 (b), we assume that these two atomic changes appear once only, the attribute CommonAttribute and the operation Commonoperation() of the class Originalclass I are moved into the superclass, and the attribute CommonAttribute and Commonoperation() of the class Originalclass 2 are deleted, in order to make the OCL expression more concise.

Traceability Links: Four traceability links have to be established: one between the classes Originalclass1 (referred to as origClass1) and RefinedClass1 (referred to as subclass1); one between the classes Originalclass2 (referred to as origClass2) and RefinedClass2 (referred to as subclass2); one between the classes Originalclass 1 and superclass (referred to as superclass); one between the classes Originalclass 2 and superclass. These traceability links are specified in the OCL expressions in Figure 85 (b).

User Help: none. 


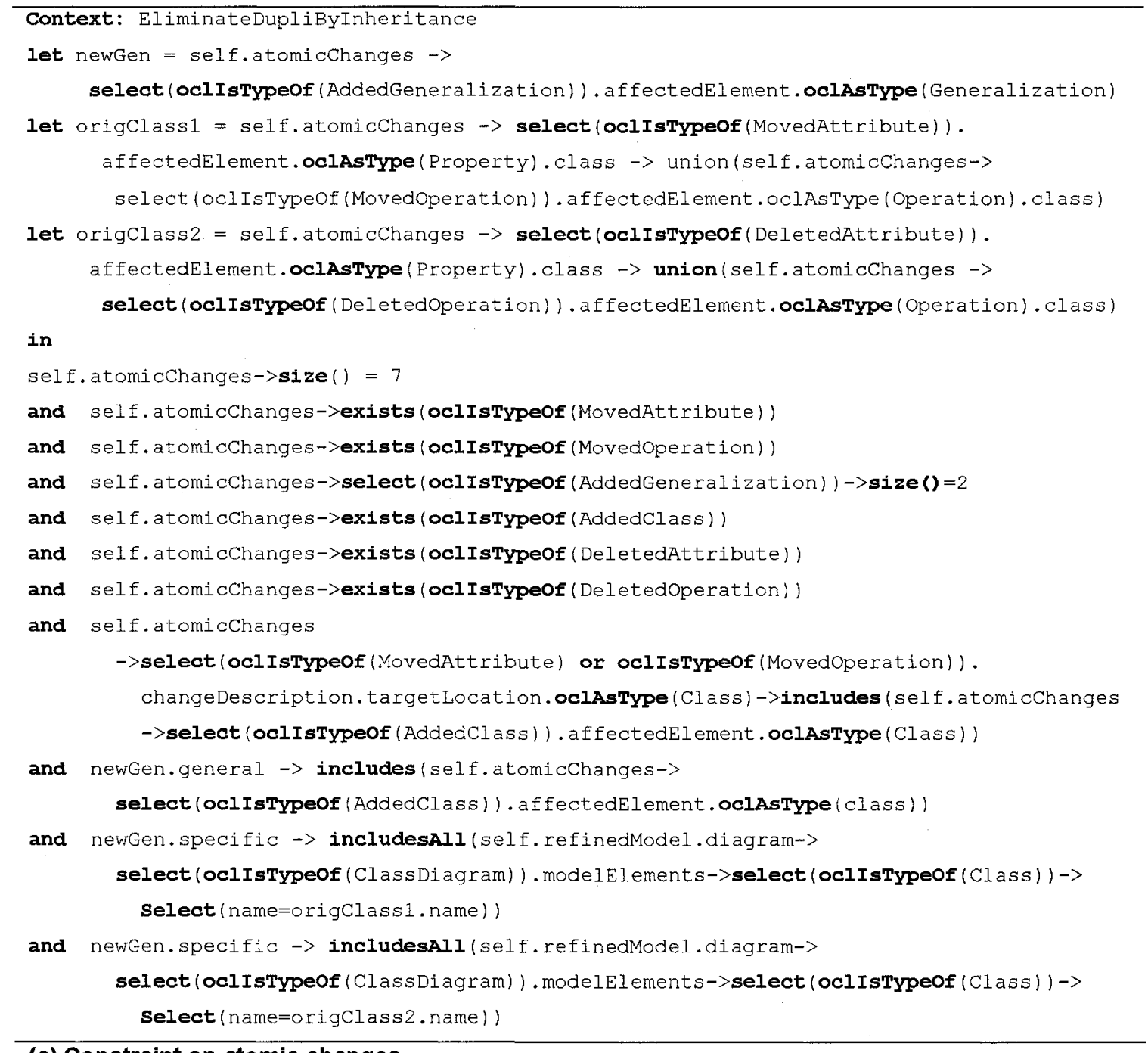

\section{(a) Constraint on atomic changes}

Figure 84 (a) Refinement EliminateDupliByInheritance (part A) 


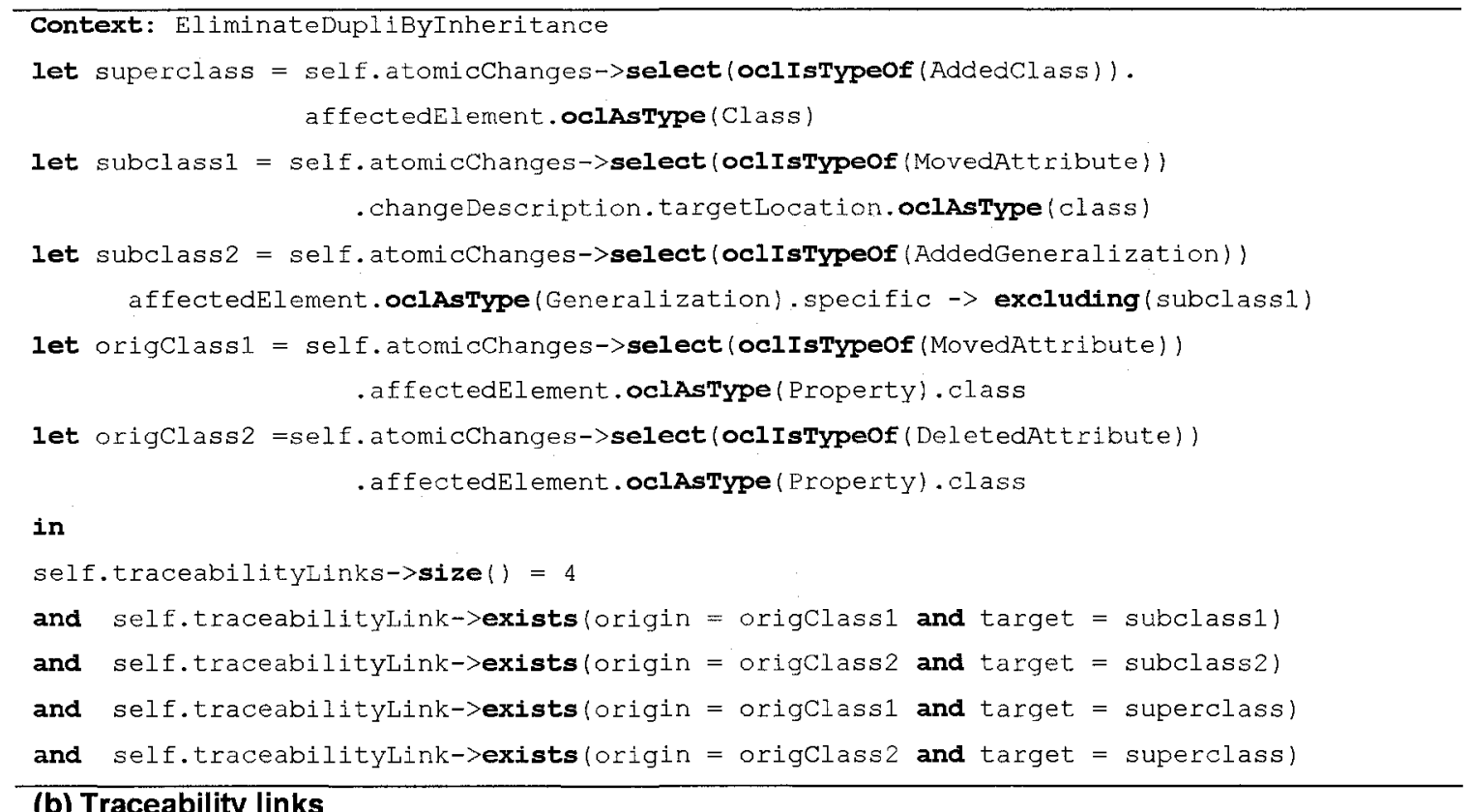

(b) Traceability links

Figure 85 (b) Refinement EliminateDupliByInheritance (part B) 


\section{E.3.6 EliminateDupliByComposition}

Decription: Extracts out the common attributes and operations of several classes, and creates a new class connecting to these classes by composition relationships to store these common attributes and operations.

Specification: As shown in Figure 86, the attribute CommonAttribute and the operation Commonoperation() of the class Originalclass 1 and OriginalClass 2 are extracted out and put into the newly added class NewClass which is connected with the class RefinedClass1 and RefinedClass 2 by composition associations. The class Refinedclass1 is refined from the class Originalclass1, and the class RefinedClass 2 is refined from the class Originalclass2.

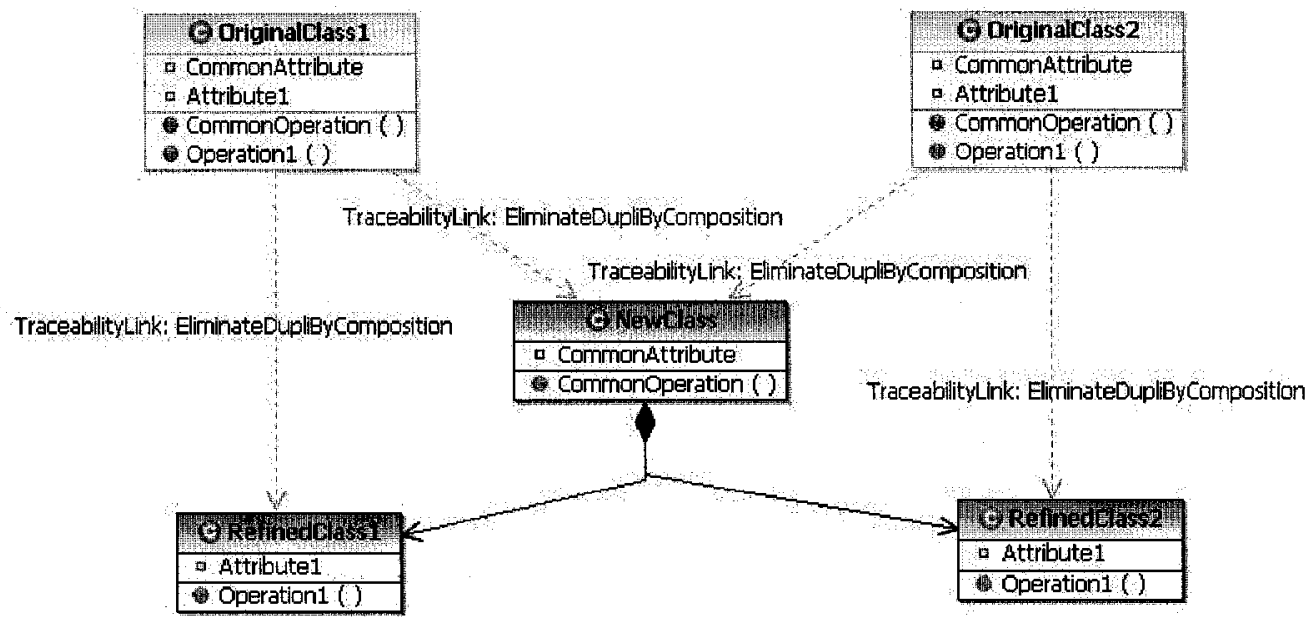

Figure 86 EliminateDupliByComposition - Specification

User's Intention: Because composition association suggests whole-part relationships. Similar attribute(s) and/or operation(s) can be extracted out to the instance of the whole (i.e. new added class).

Atomic Changes: Six kinds of atomic changes are used to derive the refinement: MovedAttribute, Movedoperation, AddedClass, AddedAssociation, DeletedAttribute, and Deletedoperation. The specification is shown as the OCL invariant for class EliminateDupliByComposition in Figure 87 (a). Note that the atomic changes DeletedAttribute and Deletedoperation may appear more than 
once because it is possible to extract out the common attribute and operation from more than two classes. In the OCL expressions in Figure 87 (a) and Figure 88 (b), we assume that these two atomic changes appear once only, the attribute commonAttribute and the operation Commonoperation() of the class Originalclass 1 are moved into the new added class, and the attribute commonAttribute and commonoperation() of the class OriginalClass 2 are deleted, in order to make the OCL expression more concise.

Traceability Links: Four traceability links have to be established: one between the classes Originalclass1 (referred to as origClass1) and RefinedClass1 (referred to as refinedClass1); one between the classes originalclass2 (referred to as origclass2) and RefinedClass2 (referred to as refinedClass2); one between the classes OriginalClass1 and NewClass (referred to as newClass); one between the classes Originalclass 2 and NewClass. These traceability links are specified in the OCL expressions in Figure 88 (b).

User Help: none. 


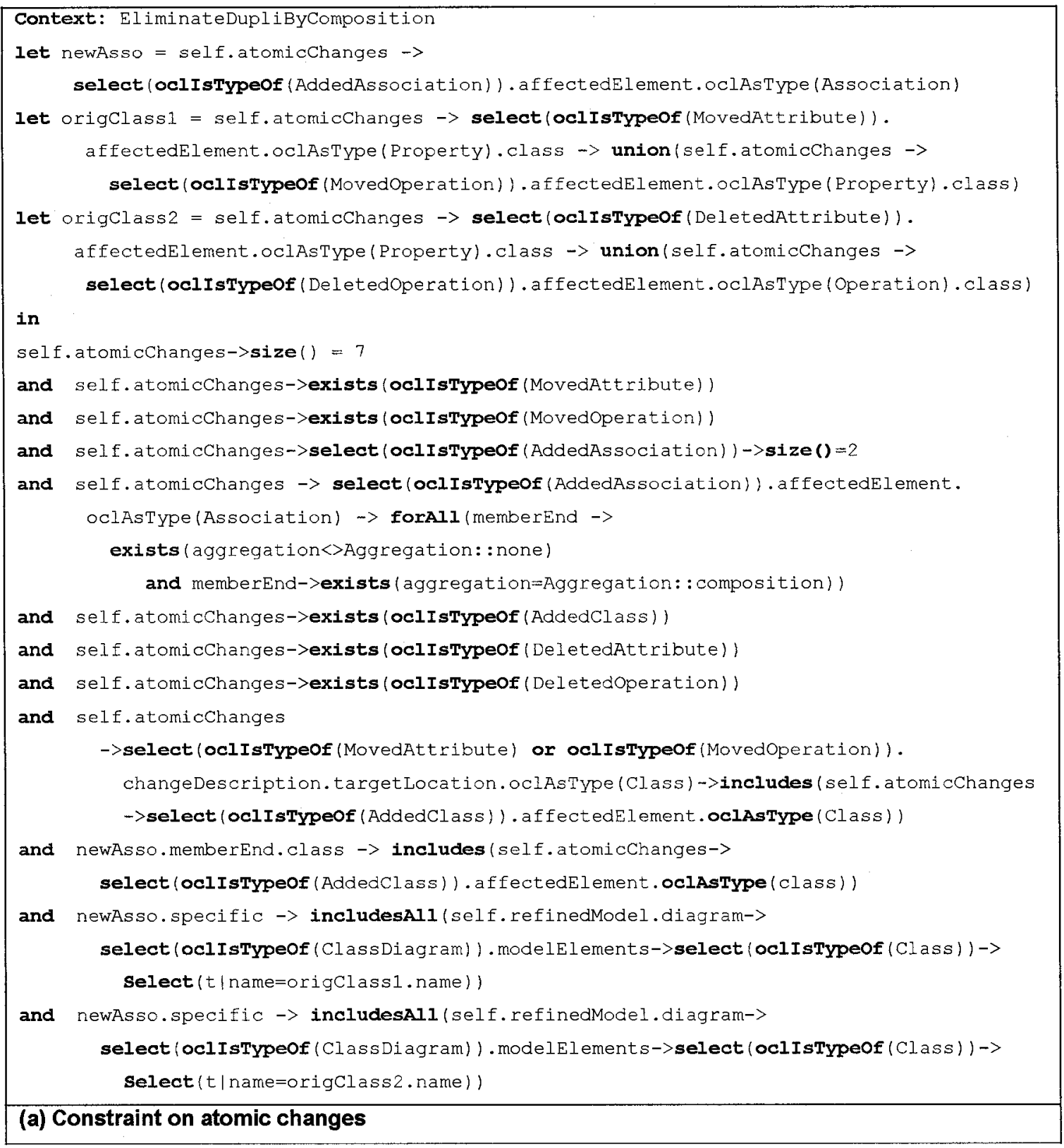

Figure 87 (a) Refinement EliminateDupliByComposition (part A) 


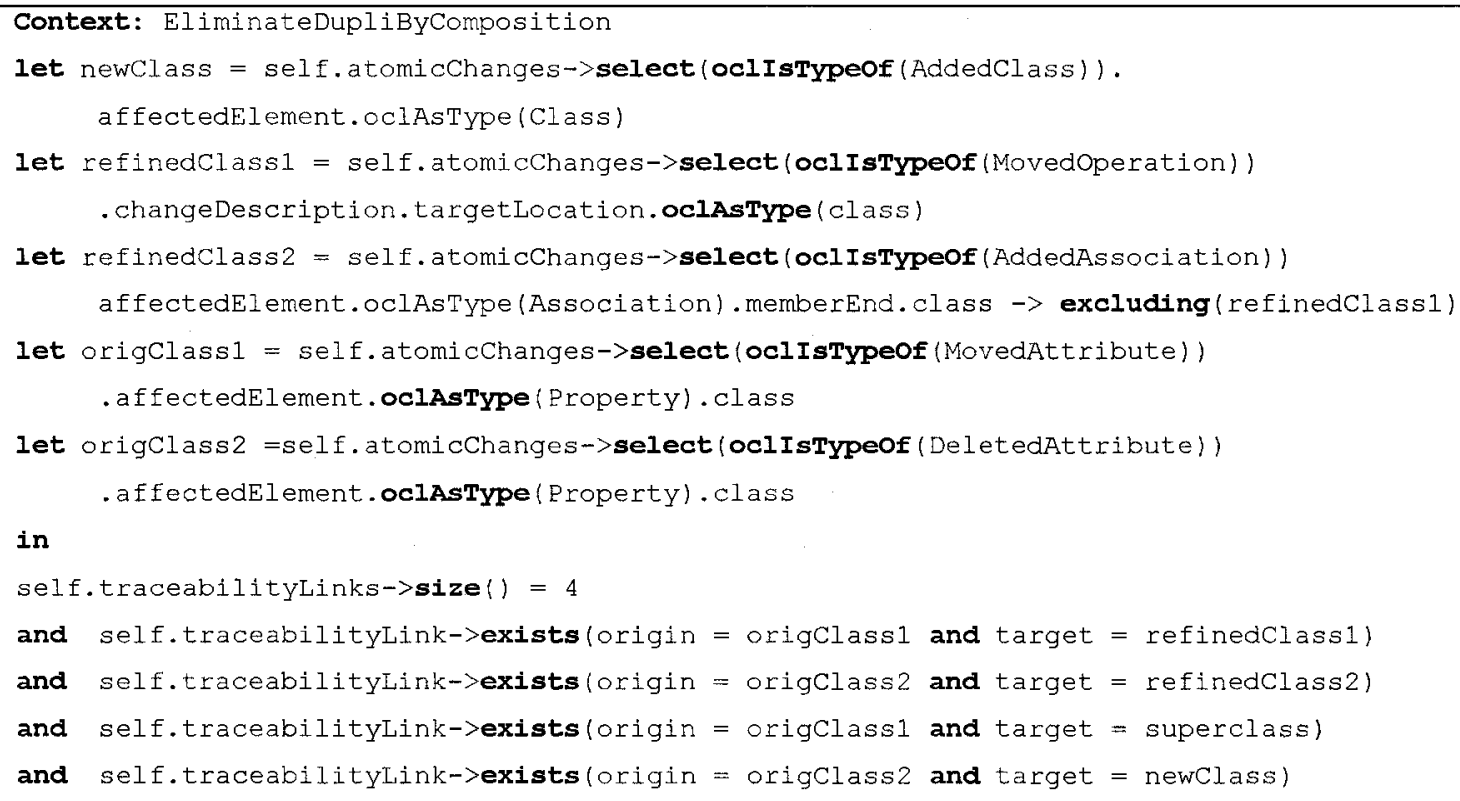

Figure 88 (b) Refinement EliminateDupliByComposition (part B) 


\section{E.3.7 SplitSubclass}

Description: Splits one of the subclasses of a superclass into two subclasses of the same superclass.

Specification: As shown in Figure 89, the subclass subclass is split into the classes RefinedSubclass and NewSubclass. The class RefinedSubclass is refined from the class subclass. The class NewSubclass is a newly added subclass. The class Superclass is refined into RefinedSuperclass. The attribute Attribute 2 and the operation Operation2() of the subclass subclass are moved into the class NewSubclass.

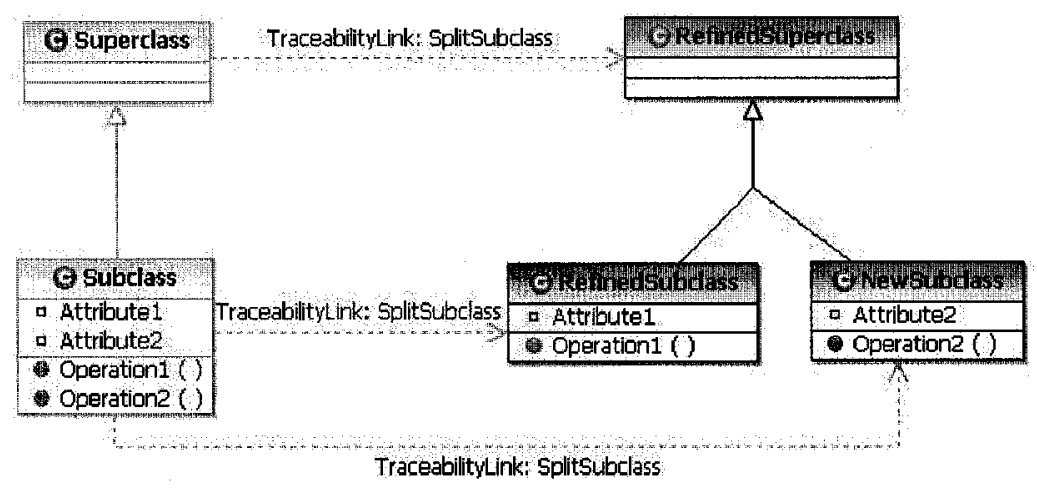

Figure 89 splitsubclass - Specification

User's Intention: A subclass takes too much responsibility which makes the hierarchy structure not clear. Splitting the responsibility and letting two or more subclasses to share it will make the generalization hierarchy clearer.

Atomic Changes: Four kinds of atomic changes are used to derive the refinement: MovedAttribute, Movedoperation, AddedClass, and AddedGeneralization. The specification is shown as the OCL invariant for class SplitSubclass in Figure 90 (a). Note that the atomic changes MovedAttribute and Movedoperation may appear more than once because it is possible that more than one attribute or operation is split to the new subclass. In the OCL expressions in Figure 90, we assume that these two atomic changes appear once only in order to make the OCL expression more concise. 
Traceability Links: Three traceability links have to be established: one between the classes Superclass (referred to as origsuper) and Refinedsuperclass (referred to as refinedsuper); one between the classes Subclass (referred to as origsub) and RefinedSubclass (referred to as refinedsub); one between the classes subclass and NewSubclass (referred to as newSub). These traceability links are specified in the OCL expressions in Figure 90 (b).

User Help: none. 


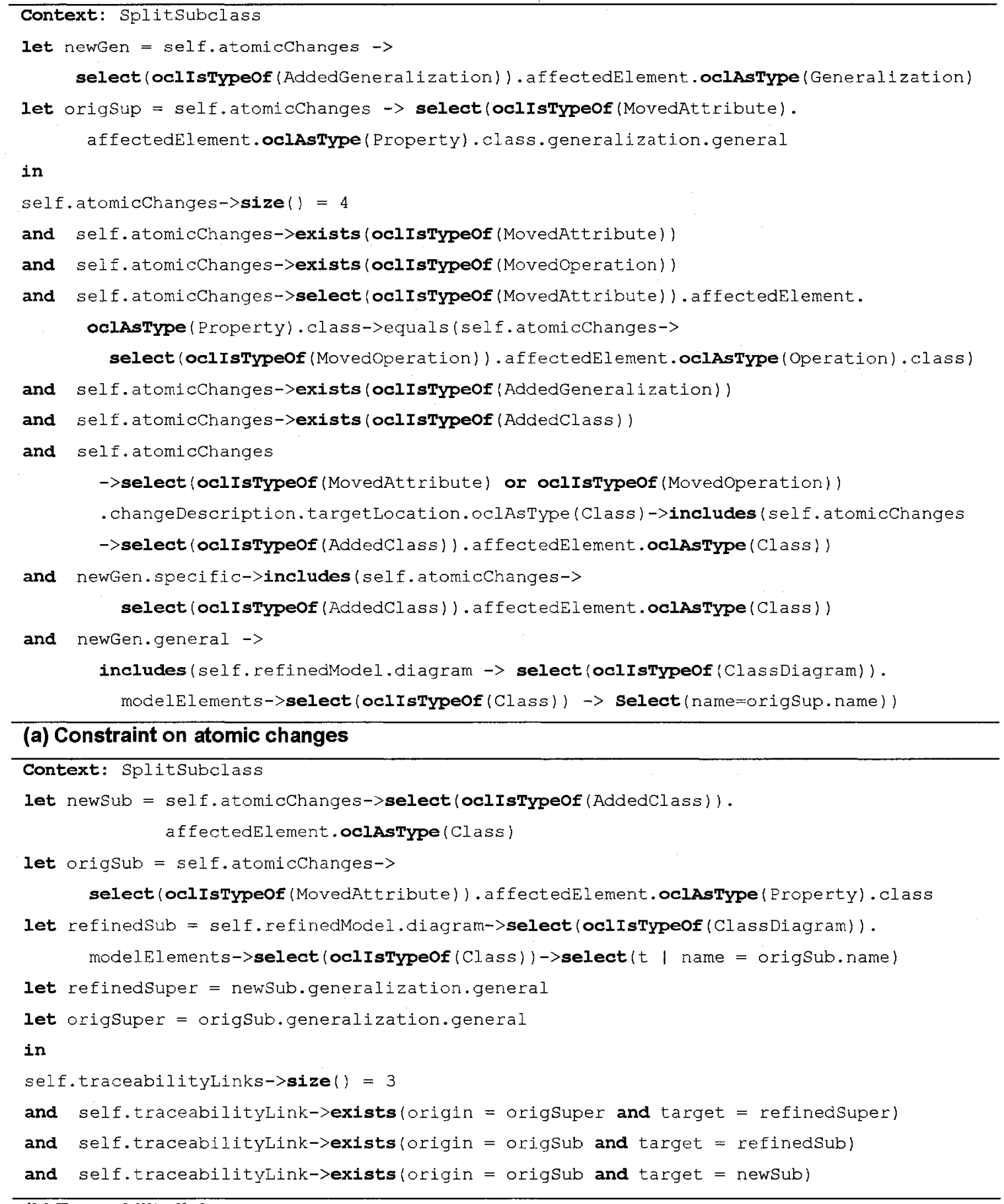

(b) Traceability links

Figure 90 Refinement Splitsubclass 


\section{E.4 Rel $\rightarrow$ Classes + Rels}

This refinement kind refines a single relationship into several classes and relationships. Figure 91 provides a refined classification involving four types of refinements.

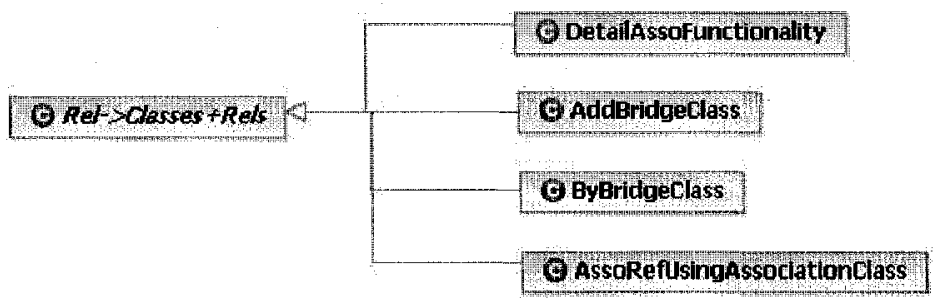

Figure 91 Taxonomy for Refinements of Type Rel->Classes+Rels

A first purpose is to detail a relationship by adding new classes and associations. DetailAssoFunctionality belongs to this type (Section E.4.1). The second possible purpose is that a new bridge class is added on the path from the one class to the other, for instance to improve structure of the model. AddBridgeClass belongs to this type (Section E.4.2). The third purpose is that an existing class is used as the bridge class. ByBridgeclass belongs to this type (Section E.4.3). The last one is that an association class is used to refine the association. AssoRefUsingAssociationclass belongs to this type (Section E.4.4).

We introduce a new class, called Path, which has four operations: exists (endA, endB), exists (endSetA, endSetB), getpaths(endA, endB), and getPaths (endSetA, endSetB). The class Path does not appear in our metamodel as it is only a helper class used to shorten our OCL expressions. The formalization of the class Path is given in Appendix F. 


\section{E.4.1 DetailAssoFunctionality}

Description: Refines an association into paths by adding new classes and relationships.

Specification: As shown in Figure 92, the association origAsso, which connects the classes originalA and originalB, is refined into a path which starts from the class RefinedA, traverses the association newAsSOAC, the class NewC and the association newAssocB, and ends at the class RefinedB. Note that the association may be refined into several paths although this case is not shown in Figure 92. The general idea for identifying such a refinement is to be able to find, in the refined class diagram, at least one path between the classes at the two ends of the association ${ }^{9}$ being deleted in the original model.

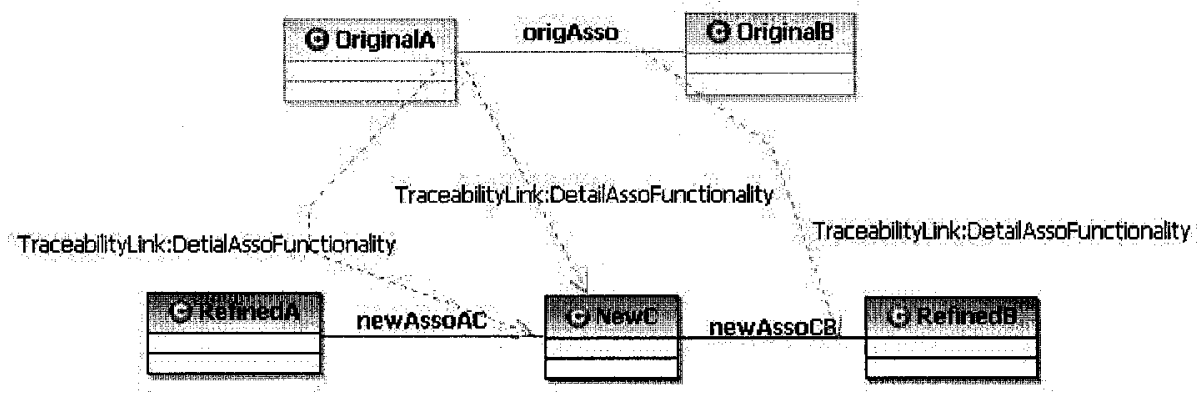

Figure 92 DetailAs soFunctionality - Specification

User's Intention: This kind of refinement happens usually when the association being refined in the original model has to be detailed by introducing more classes and associations along with the evolution of model.

Atomic Changes: As specified in Figure 93 (a), such a refinement is at least made of one DeletedAssociation atomic change: the deleted association (in the original model) is referred to as origAsso. The next let expressions are used to identify the two ends of the deleted association in the original model (origA and origB) and how they may have been refined (refA and refB). Last, we use operation exists() of class Path to identify

\footnotetext{
${ }^{9}$ In this thesis, we assume associations are binary, although n-ary associations can be handled following the same principles.
} 
whether there is a path between $r e f A$ and $r e f B$ in the refined model. The operation exists (endSetA, endSetB) is used in Figure 93 (a) since refA and refB are sets in this case.

Traceability Links: Figure 93 (b) specifies how traceability links are established for refinement DetailAssoFunctionality. For each path that we can find in the refined model between refA and refB (we use operation getPaths (refA, refB) of class Path), we establish a traceability link between the removed association origAsso (in the original model) and each element of the path.

User Help: The user's help is required because we have to ask the user to indicate which path(s) are the path(s) actually refining the deleted association. 


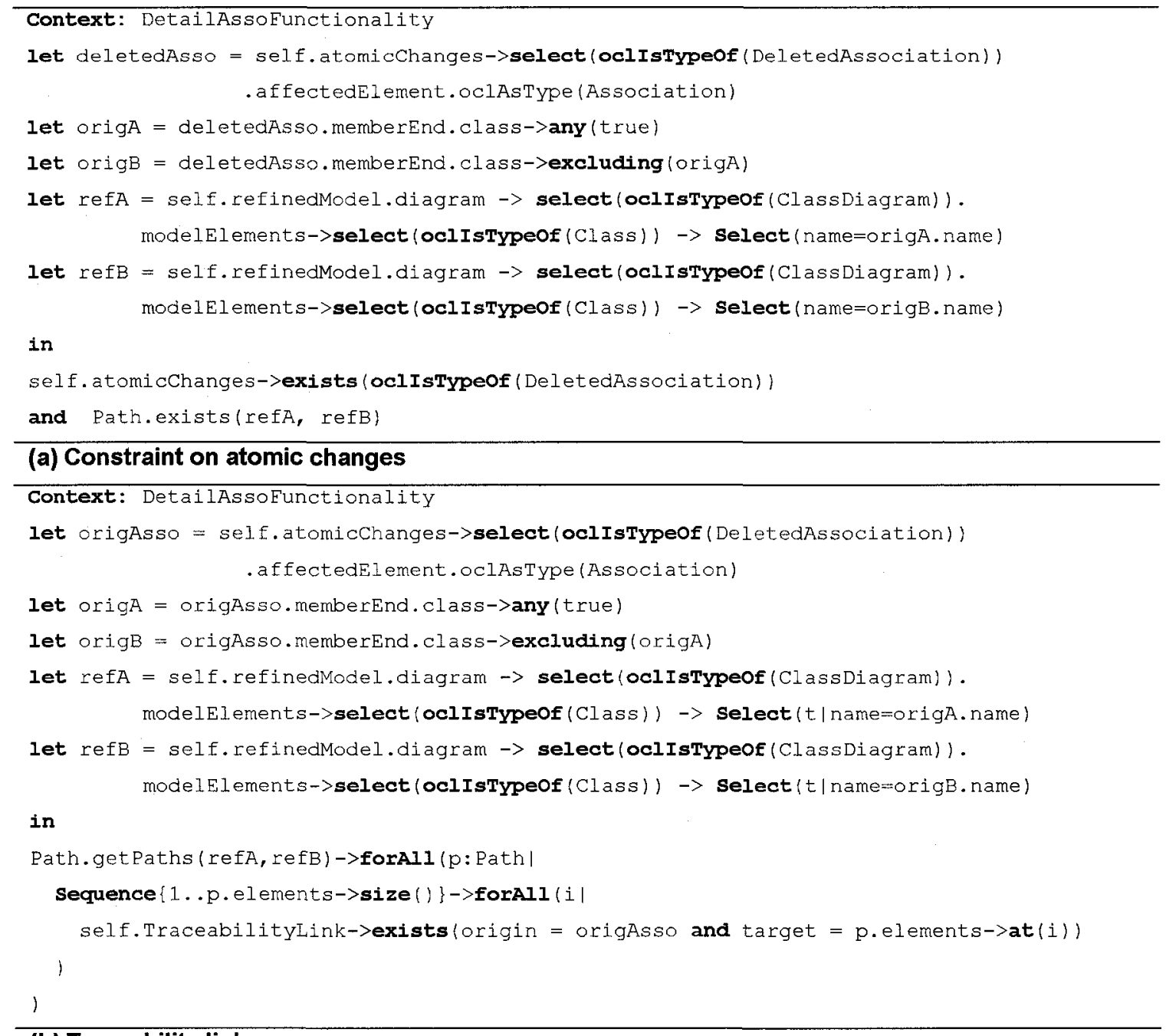

(b) Traceability links

Figure 93 Refinement DetailAssoFunctionality

Example: Figure 94 and Figure 95 provide an example which is adapted from [33]. The association between the classes ATM and Customer is refined into five paths:

1. $\mathrm{ATM} \rightarrow$ CardReader $\rightarrow$ Card $\rightarrow$ Customer

2. ATM $\rightarrow$ Console $\rightarrow$ Customer

3. ATM $\rightarrow$ Slot $\rightarrow$ Customer

4. $\mathrm{ATM} \rightarrow \mathrm{Dispenser} \rightarrow$ Customer

5. ATM $\rightarrow$ Transaction $\rightarrow$ Customer 


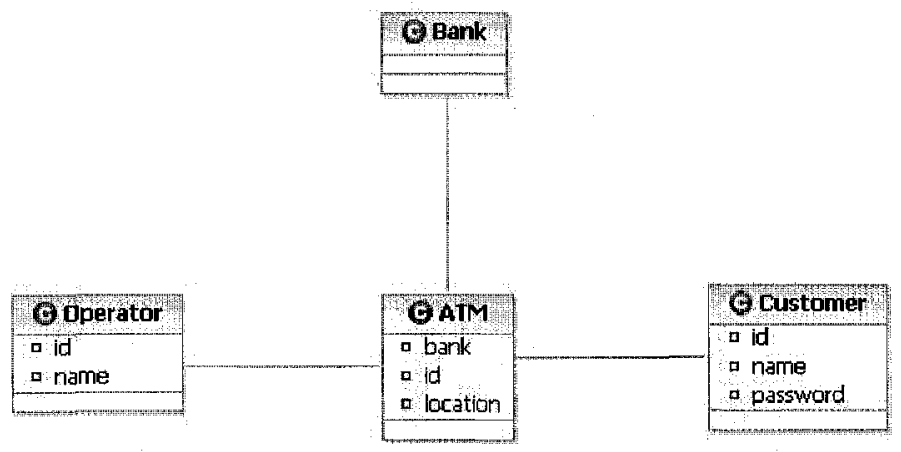

Figure 94 Example DetailAssoFunctionality - the original class diagram

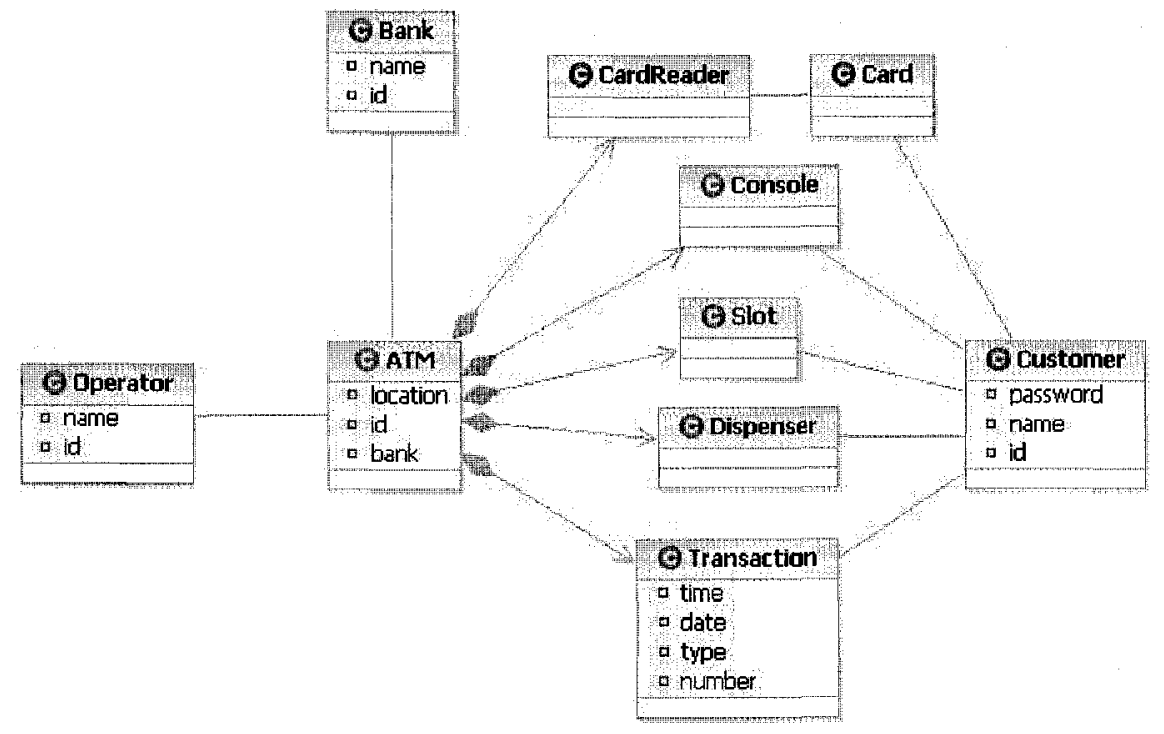

Figure 95 Example DetailAssoFunctionality - the refined class diagram 


\section{E.4.2 AddBridgeClass}

Description: Adds a new bridge class between the association ends of the association being refined in order to improve the model structure.

Specification: As shown in Figure 96, the new bridge class NewBridgeClass is added between the classes RefinedClassA and RefinedClassB. The class RefinedClassA is refined from the class OriginalclassA and the class RefinedClassB is refined from the class OriginalClassB.

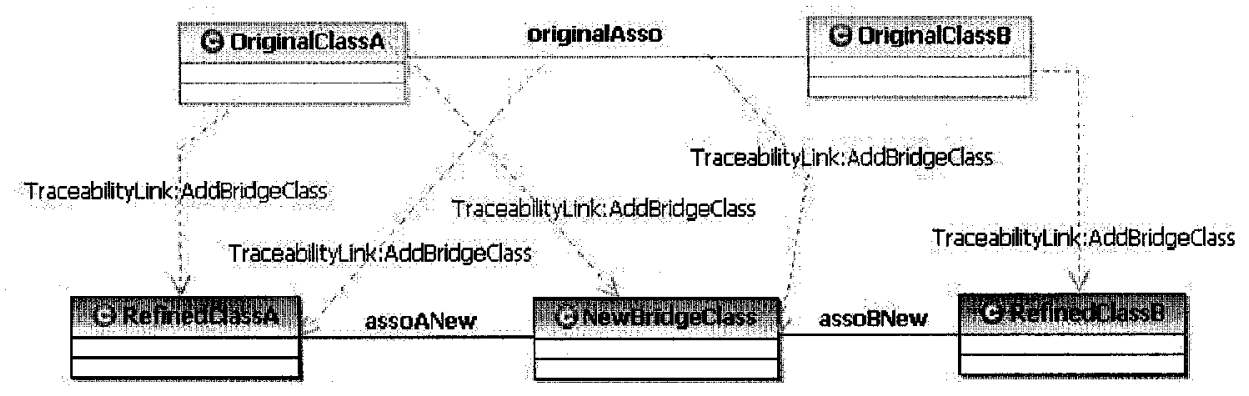

Figure 96 AddBridgeClass - Specification

User's Intention: See description.

Atomic Changes: Three kinds of atomic changes are used to derive the refinement: AddedClass, AddedAssociation, and DeletedAssociation. The specification is shown as the OCL invariant for class AddBridgeClass in Figure 97 (a). Note that the atomic changes AddedAssociation appears twice in order to connect the newly added class with the existing two classes. One of the association ends of the originalAsso may be relocated to class NewBridgeclass. In such situation, an atomic change RelocatedAssociation appears instead of a DeletedAssociation atomic change and an AddedAssociation atomic change. In the OCL expressions in Figure 97, we assume that no atomic change RelocatedAssociation appears, in order to make the OCL expression more concise.

Traceability Links: Five traceability links have to be established: 
- one between the classes OriginalclassA (referred to as origClassA) and RefinedClassA (referred to as refinedClassA);

- one between the classes OriginalClassB (referred to as origClassB) and RefinedClassB (referred to as refinedClassB);

- one between the association originalAsso (referred to as origAsso) and the class NewBridgeclass (referred to as newBridgeClass);

- one between the associations originalAsso and assoANew (referred to as assOANew);

- one between the associations originalAsso and assoBNew (referred to as assoBNew).

These traceability links are specified in the OCL expressions in Figure 97 (b).

User Help: none. 


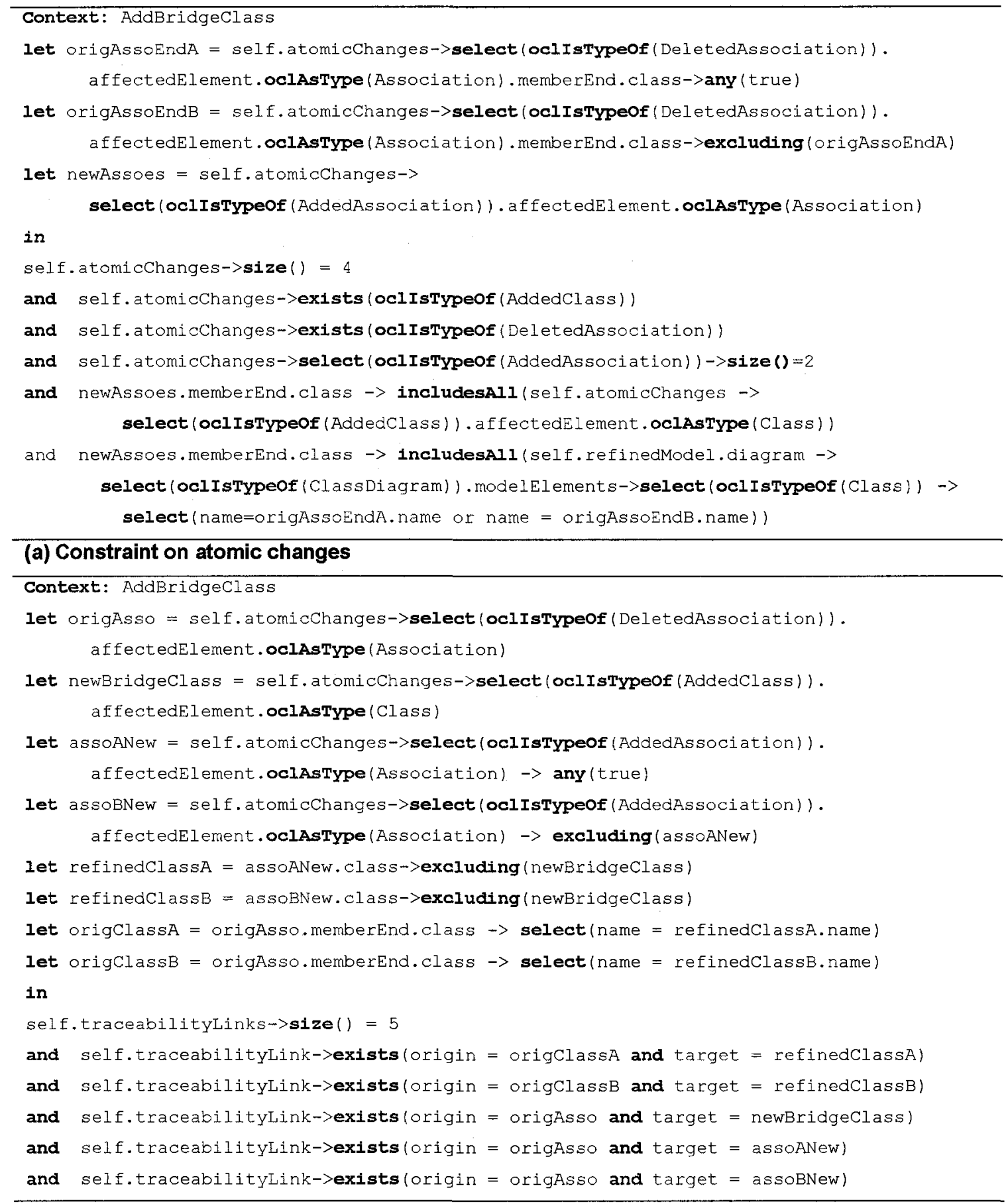

(b) Traceability links

Figure 97 Refinement AddBridgeclass 


\section{E.4.3 ByBridgeClass}

Description: Uses an existing class as the bridge class between the association ends of the association being refined in order to improve the model structure.

Specification: As shown in Figure 98, the bridge class Bridgeclass is used to associate the classes RefinedClassA and RefinedClassB. The class RefinedClassA is refined from the class OriginalClassA and the class RefinedClassB is refined from the class OriginalclassB. The association originalAsso is refined into the path which starts from the class RefinedClassA, traverses the association assoANew, the class BridgeClass and the association assoB, and finally ends at the class RefinedClassB.

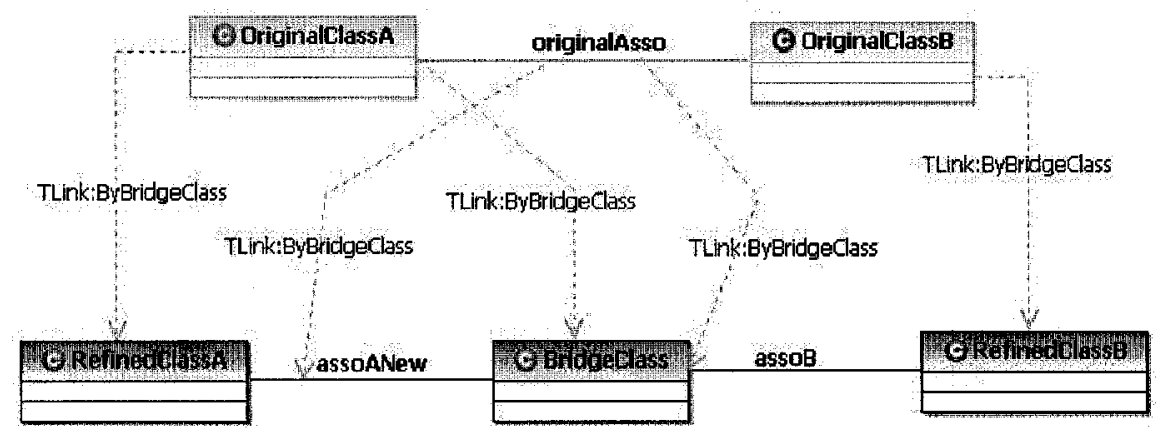

Figure 98 ByBridgeclass - Specification

User's Intention: See description.

Atomic Changes: The specification is given in Figure 99 (a). Two kinds of atomic changes are used to derive the refinement: AddedAssociation and DeletedAssociation. Note that the atomic change AddedAssociation should appear once or two in order to connect the classes RefinedClassA and RefinedClassB with the class Bridgeclass. The association originalAsso may be relocated to class NewBridgeClass. In such situation, an atomic change RelocatedAssociation appears instead of a DeletedAssociation atomic change and an AddedAssociation atomic change. In the OCL expressions in Figure 99, we assume that no atomic change 
RelocatedAssociation appears, in order to make the OCL expression more concise. We also assume that the association as $\mathrm{SOB}$ exists already and the association as soANew is a newly added association.

Traceability Links: Figure 99 (b) specifies how traceability links are established for refinement ByBridgeClass. For each path that we can find in the refined model between refA and refB (we use operation getPaths (refA, refB) of class $P a t h$ ), we establish a traceability link between the removed association origAsso (in the original model) and each element of the path.

User Help: The user's help is required because we have to ask the user to distinguish this

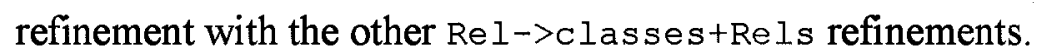




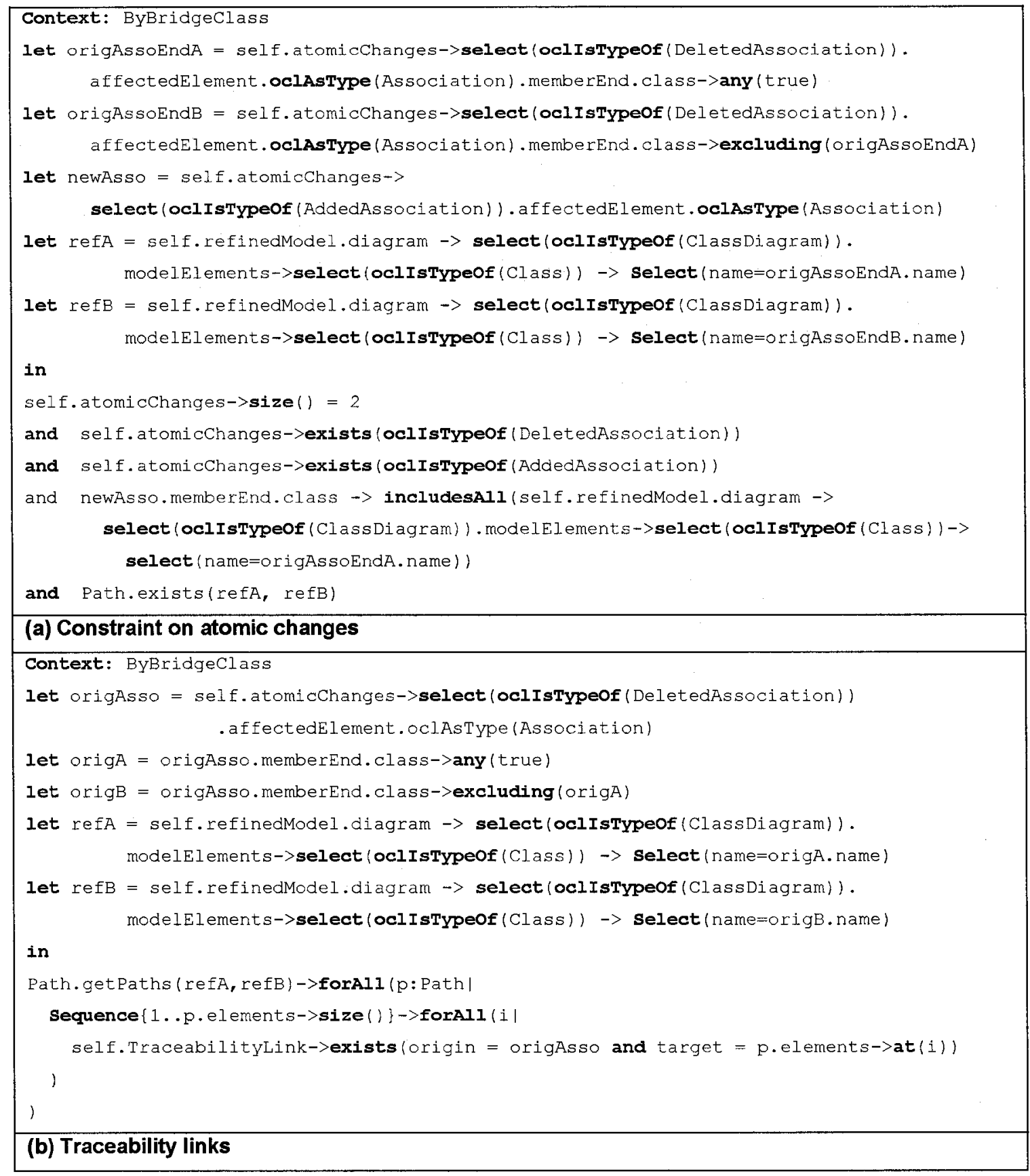

Figure 99 Refinement ByBridgeClass

Example: Figure 100 and Figure 101 provide an example. The association between the classes Session and CustomerConsole is refined into the path traversing the existing class ATM which is the bridge class. 


OATM $\quad$ Obession $\quad$ OCustomerconsole

Figure 100 Example ByBridgeclass - the original class diagram

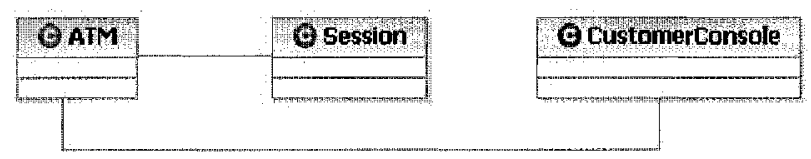

Figure 101 Example ByBridgeClass - the refined class diagram 


\section{E.4.4 AssoRefUsingAssociationEnd}

Description: Transforms an association into an association class.

Specification: As shown in Figure 102, the association class NewAssociationclass is used to associate the classes RefinedClassA and RefinedClassB. The class RefinedClassA is refined from the class originalclassA and the class RefinedClassB is refined from the class OriginalClassB.

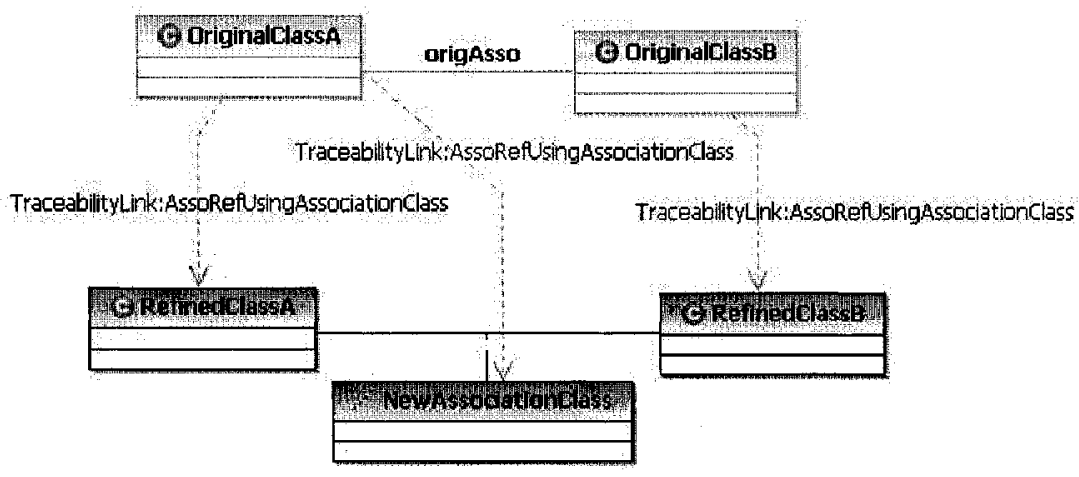

Figure 102 AssoRefUsingAssociationclass - Specification

User's Intention: An association is refined to have its own set of features; that is, features that do not belong to any of the connected classes but rather to the association itself.

Atomic Changes: Two kinds of atomic changes are used to derive the refinement: AddedAssociationClass and DeletedAssociation. The specification is shown as the OCL invariant for class AssoRefusingAssociationclass in Figure 103 (a).

Traceability Links: Three traceability links have to be established: one between the classes OriginalClassA (referred to as origClassA) and RefinedClassA (referred to as refinedClassA); one between the classes OriginalClassB (referred to as origClassB) and RefinedClassB (referred to as refinedClassB); one between the association origAsso (referred to as origAsso) and the association class 
NewAssociationclass (referred to as newAssoclass). These traceability links are specified in the OCL expressions in Figure 103 (b).

\section{User Help: none.}

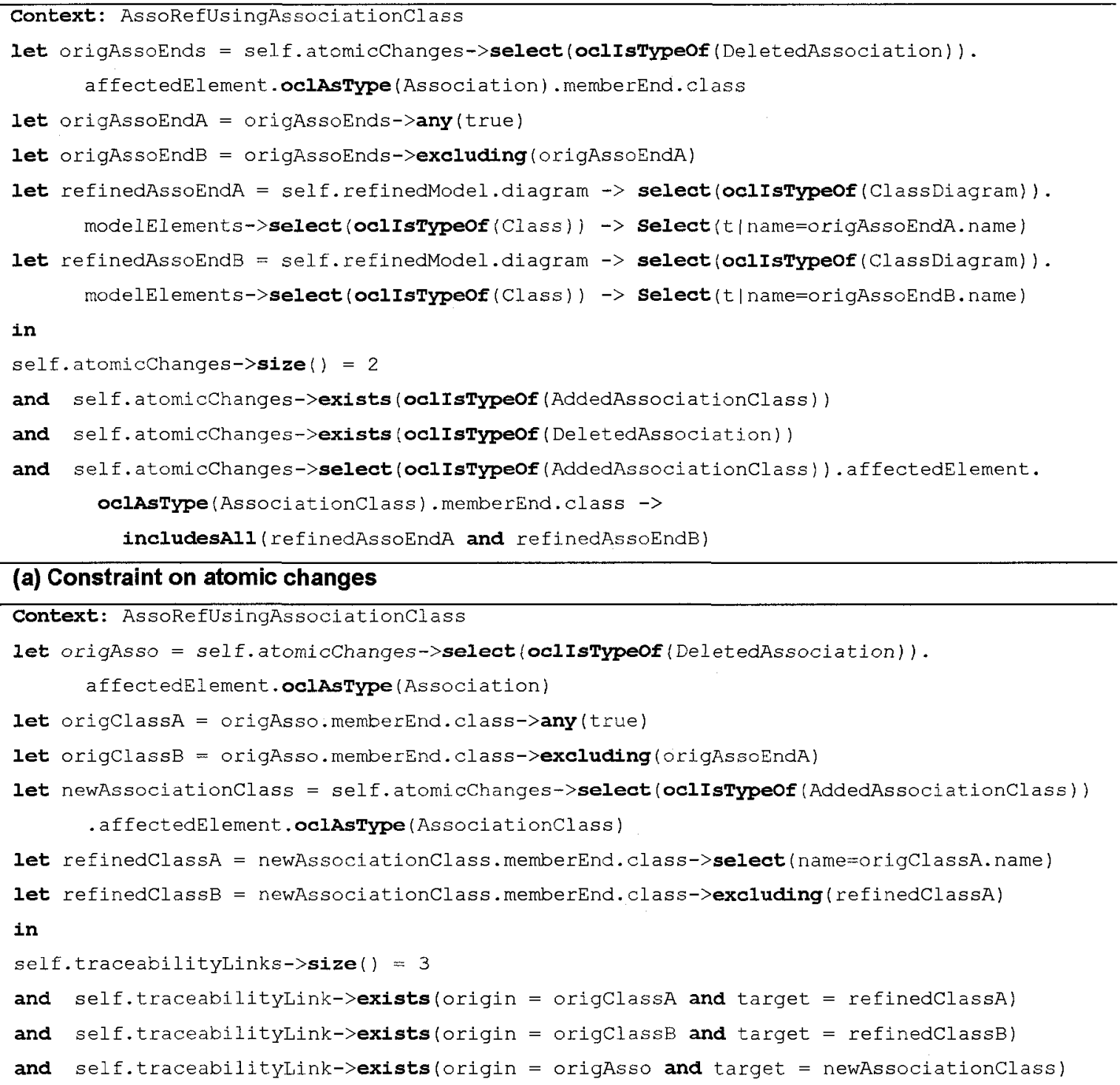

Figure 103 Refinement AssoRefUsingAssociationClass 


\section{E.5 Classes+Rels $\rightarrow$ Rel}

This refinement refines several classes and relationships into a single relationship. Figure 104 provides a refined classification involving one type of refinements (Section E.5.1).

\begin{tabular}{|c|c|}
\hline 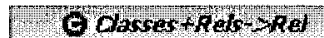 & O ReplaceAssociationClasswithasso \\
\hline
\end{tabular}

Figure 104 Taxonomy for Refinements of Type Classes+Rels->Rel

\section{E.5.1 ReplaceAssociationClassWithAsso}

Description: Replaces an association class with an association. The refinement is the opposite of the refinement AssoRefUsingAssociationclass.

Specification: As shown in Figure 105, the association class OrigAssociationclass is refined into the association refinedAsso which associates the classes RefinedClassA and RefinedClassB. The class RefinedClassA is refined from the class OriginalclassA and the class RefinedClassB is refined from the class OriginalclassB.

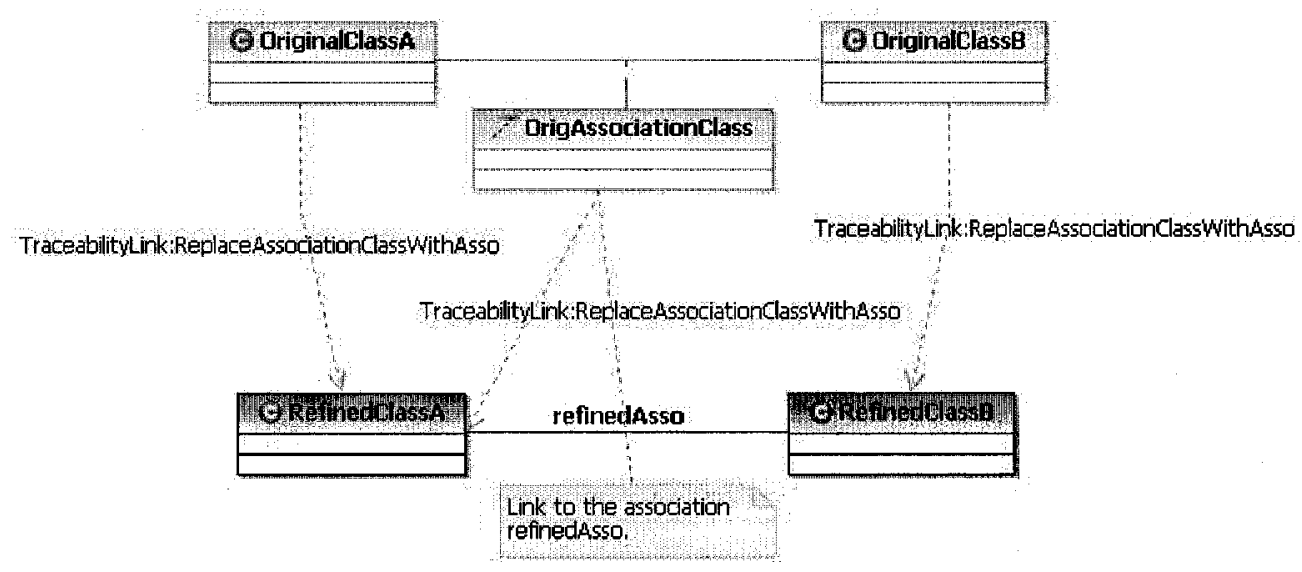

Figure 105 ReplaceAssociationClasswithAsso - Specification

User's Intention: An association class appears to be unnecessary (e.g., no attributes or attributes that are not specific to the association). 
Atomic Changes: Two kinds of atomic changes are used to derive the refinement: DeletedAssociationclass and AddedAssociation. The specification is shown as the OCL invariant for class ReplaceAssociationclasswithAsso in Figure 106 (a).

Traceability Links: Three traceability links have to be established: one between the classes OriginalClassA (referred to as origClassA) and RefinedClassA (referred to as refinedClassA); one between the classes OriginalclassB (referred to as origClassB) and RefinedClassB (referred to as refinedClassB); one between the association class origAssociationclass (referred to as origAssoClass) and the association refinedAsso (referred to as newAsso). These traceability links are specified in the OCL expressions in Figure 106 (b).

User Help: none. 


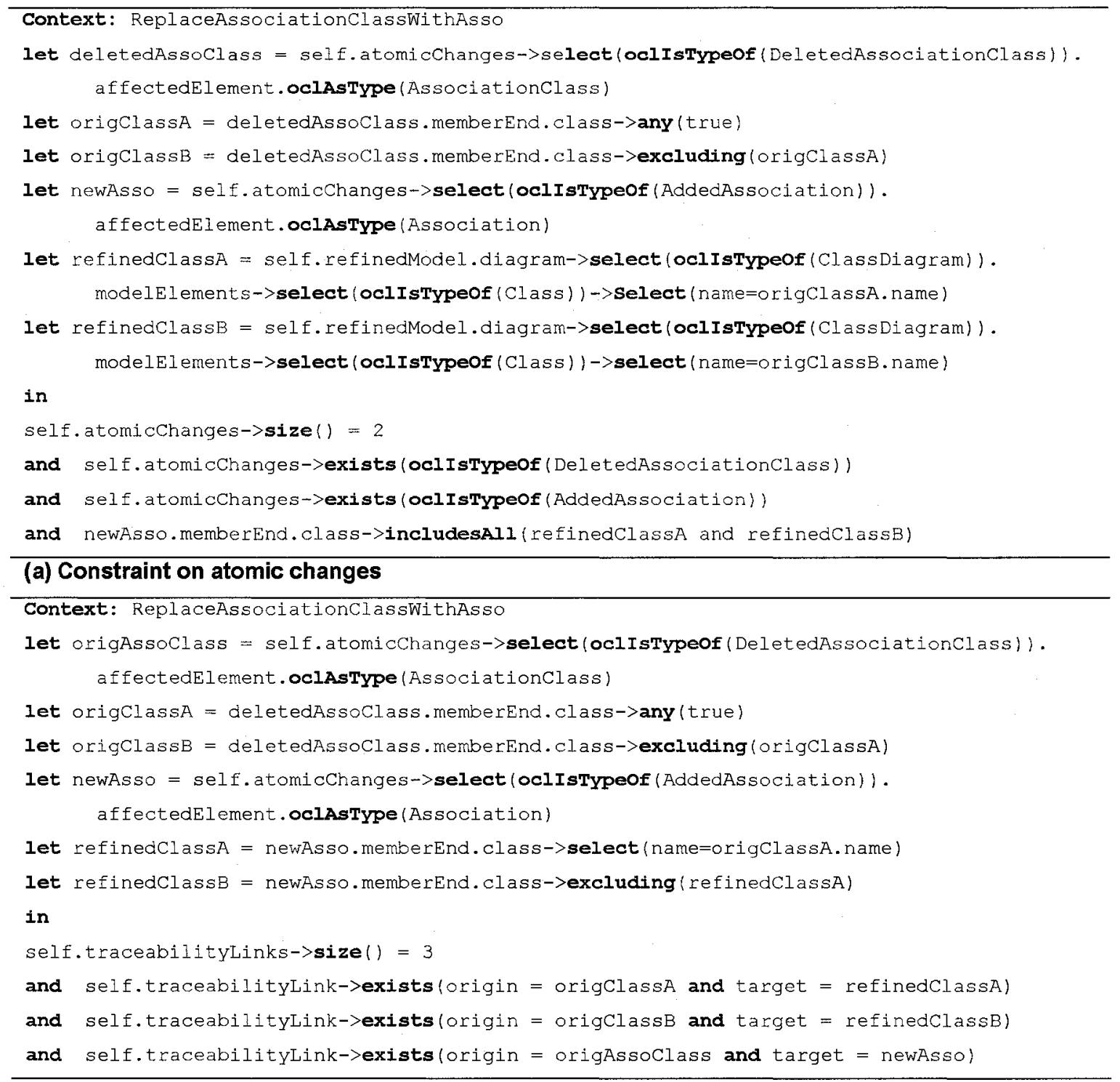

(b) Traceability links

Figure 106 Refinement ReplaceAssociationclassWithAsso 


\section{E.6 Rels $\rightarrow$ Rel}

This refinement refines several relationships into a single relationship. This type of refinement is not common, as no class is included in the refinement. Figure 107 provides a refined classification involving one type of refinements (Section E.6.1).

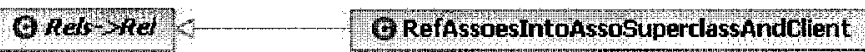

Figure 107 Taxonomy for Refinements of Type Rels $->\operatorname{Re} 1$

\section{E.6.1 RefAssoesIntoAssoSuperclassAndClient}

Description: Refines two associations between two subclasses and a client class into a single association between the superclass of these two subclasses and the client class.

Specification: As shown in Figure 108, the associations origAsso1 and origAsso2 are refined into the association refinedAsso.

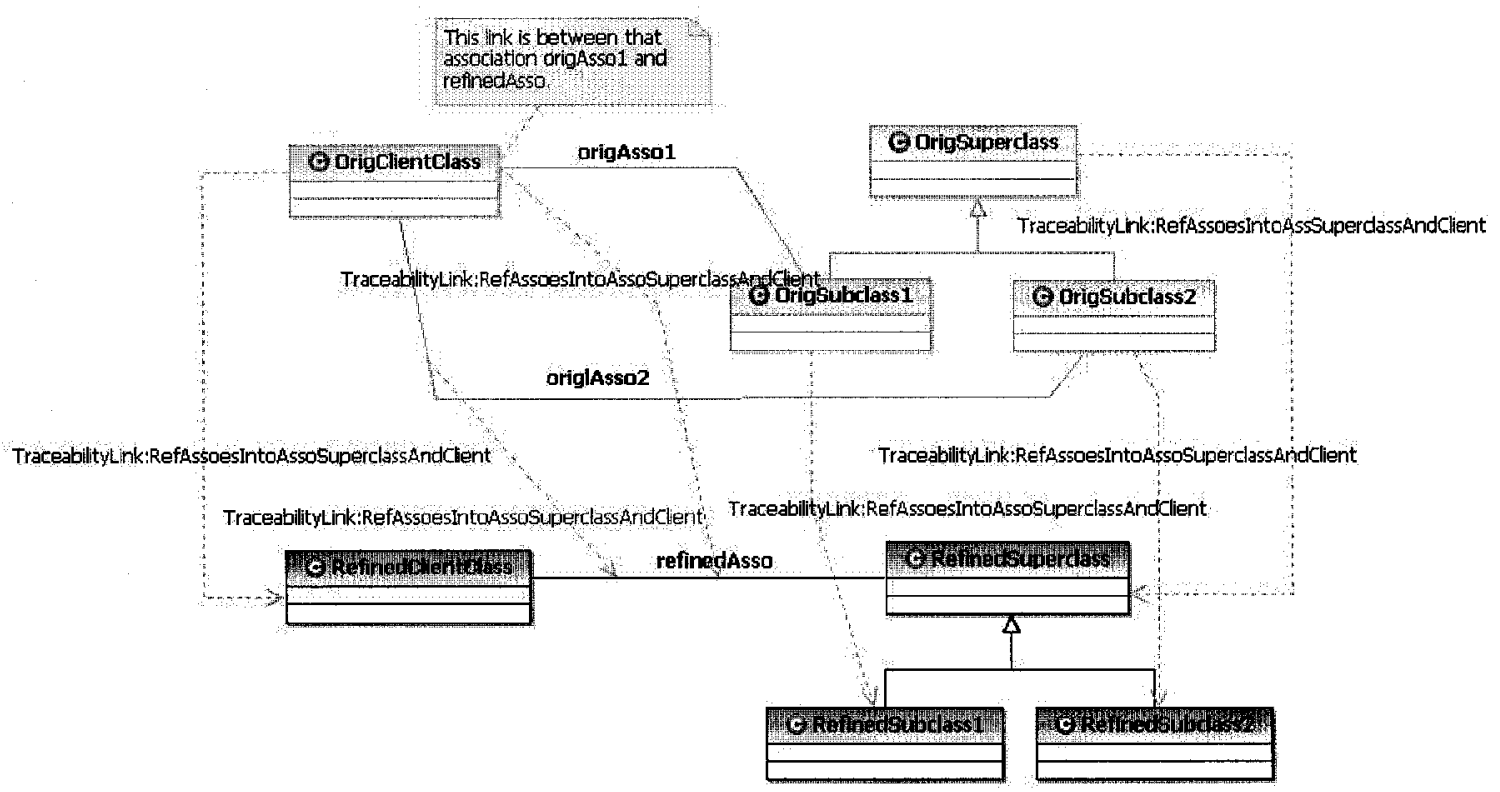

Figure 108 RefAssoes IntoAssoSuperclassAndClient - Specification

User's Intention: Two associations between a client class and two subclasses can be generalized to one association between the client class and the superclass of these two subclasses. 
Atomic Changes: Two kinds of atomic changes are used to derive the refinement: RelocateAssociation and DeletedAssociation. Note that it is possible that the two original associations between the client class and the two subclasses are all deleted (through two DeletedAssociation atomic changes) and a new association between the client and superclasse is added. We assume the former situation. The specification is shown in Figure 109.

Traceability Links: Six traceability links have to be established:

- one between the classes origsuperclass (referred to as origsuper) and RefinedSuperclass (referred to as refinedSuper);

- one between the classes OrigSubclass1 (referred to as origSub1) and RefinedSubclassl (referred to as refinedClass1);

- one between the classes Origsubclass2 (referred to as origsub2) and RefinedSubclass2 (referred to as refinedsub2);

- one between the classes origclentclass (referred to as origclient) and RefinedClentclass (referred to as refinedClient);

- one between the association origAssol (referred to as origAssol) and the association refinedAsso (referred to as refinedAsso);

- one between the association origAsso2 (referred to as origAsso2) and the association refinedAsso.

These traceability links are specified in the OCL expressions in Figure 110.

\section{User Help: none.}




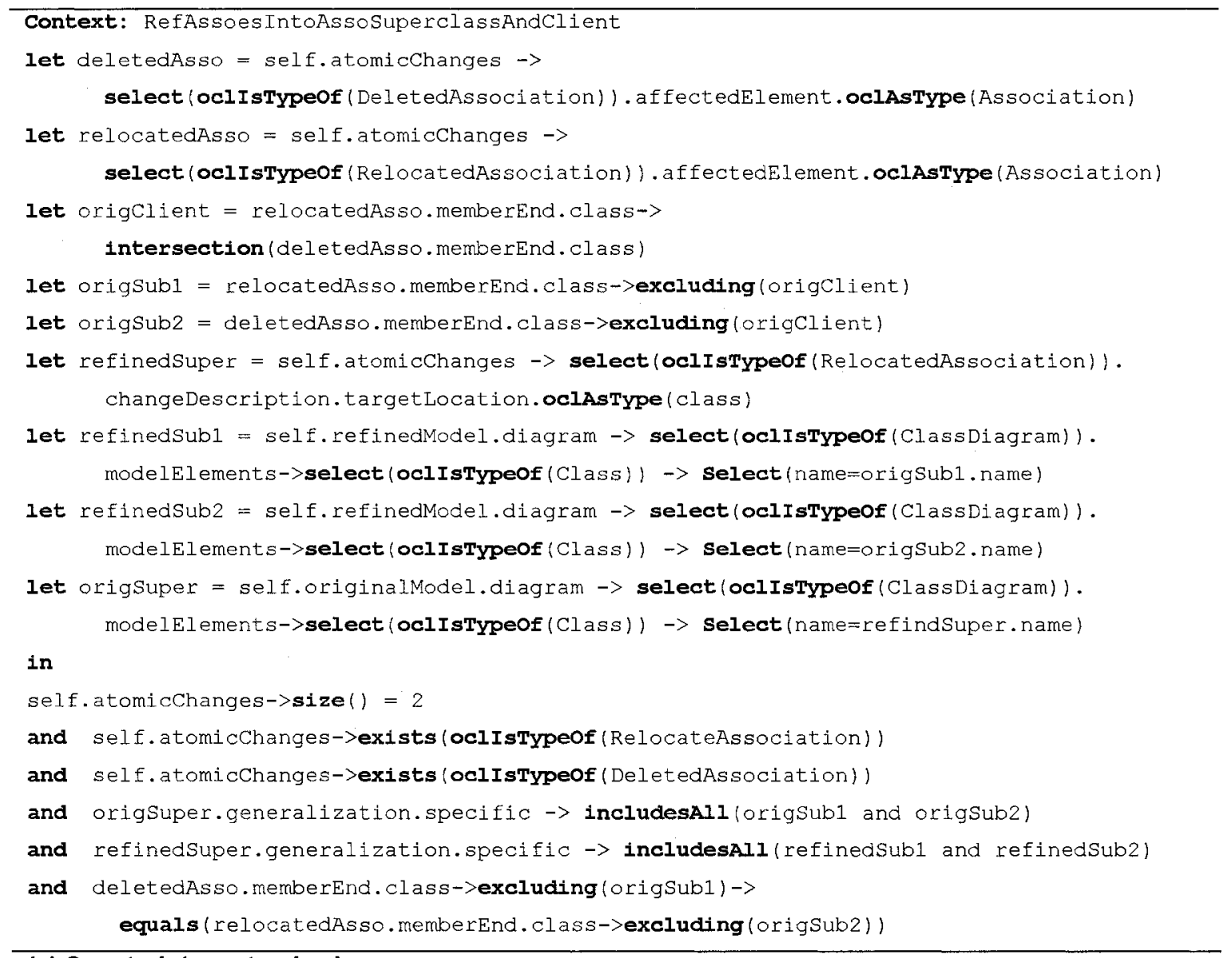

\section{(a) Constraint on atomic changes}

Figure 109 Refinement RefAssoes IntoAssoSuperclassAndClient (part A) 


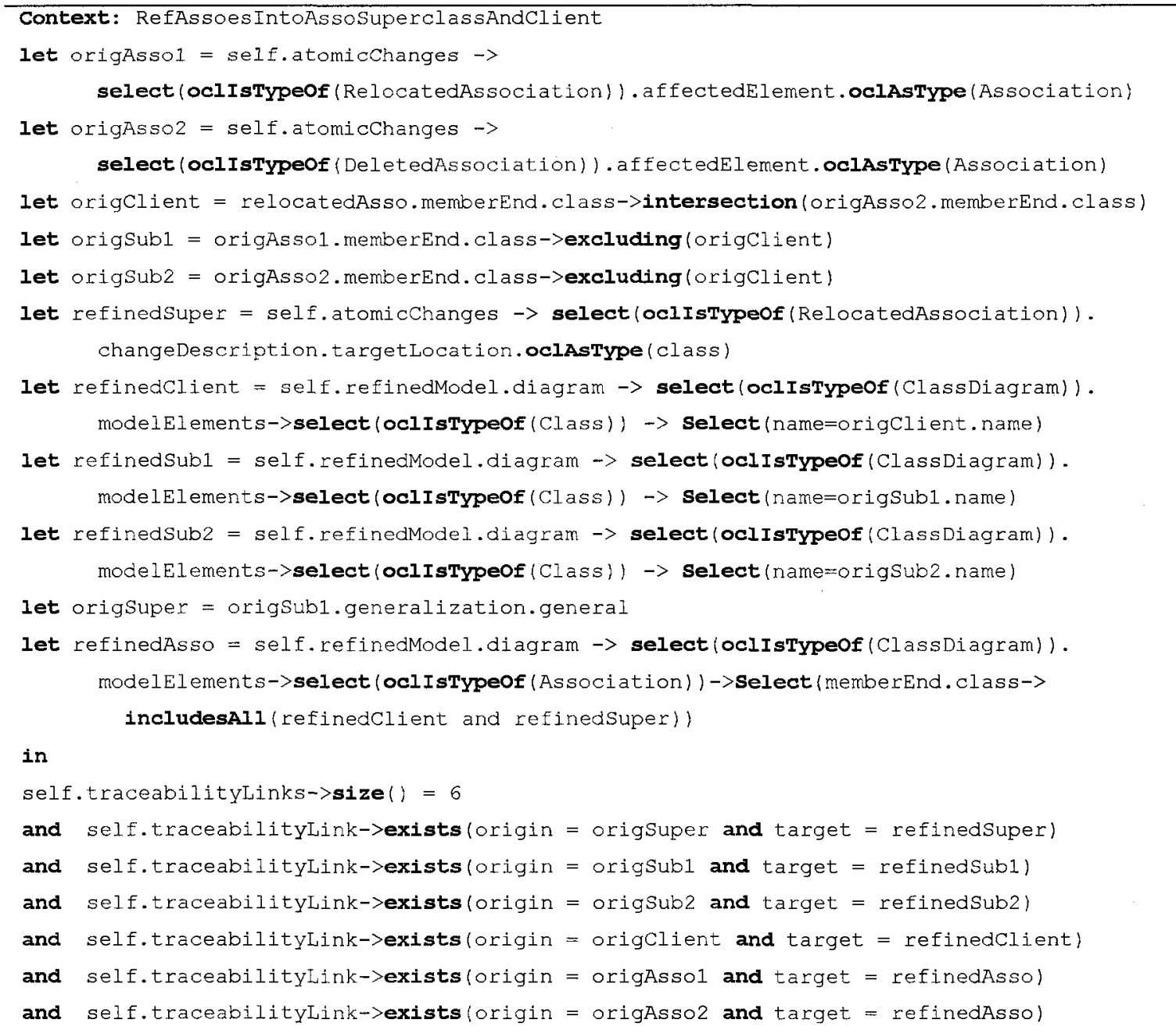

\section{(b) Traceability links}

Figure 110 Refinement RefAssoes IntoAssoSuperclassAndClient (part B) 


\section{E.7 Rel->Rels}

This refinement refines a single relationship into several relationships. This type of refinement is not common, as no class is included into the refinement. Figure 111 provides a refined classification involving one type of refinements (Section E.7.1).

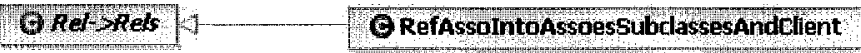

Figure 111 Taxonomy for Refinements of Type Rel->Rels

\section{E.7.1 RefAssoIntoAssoesSubclassesAndClient}

Description: Refines the association between a superclass and a client class into several associations between several subclasses of this superclass and the client class.

Specification: As shown in Figure 112, the association origAsso is refined into the associations refinedAsso and newAsso.

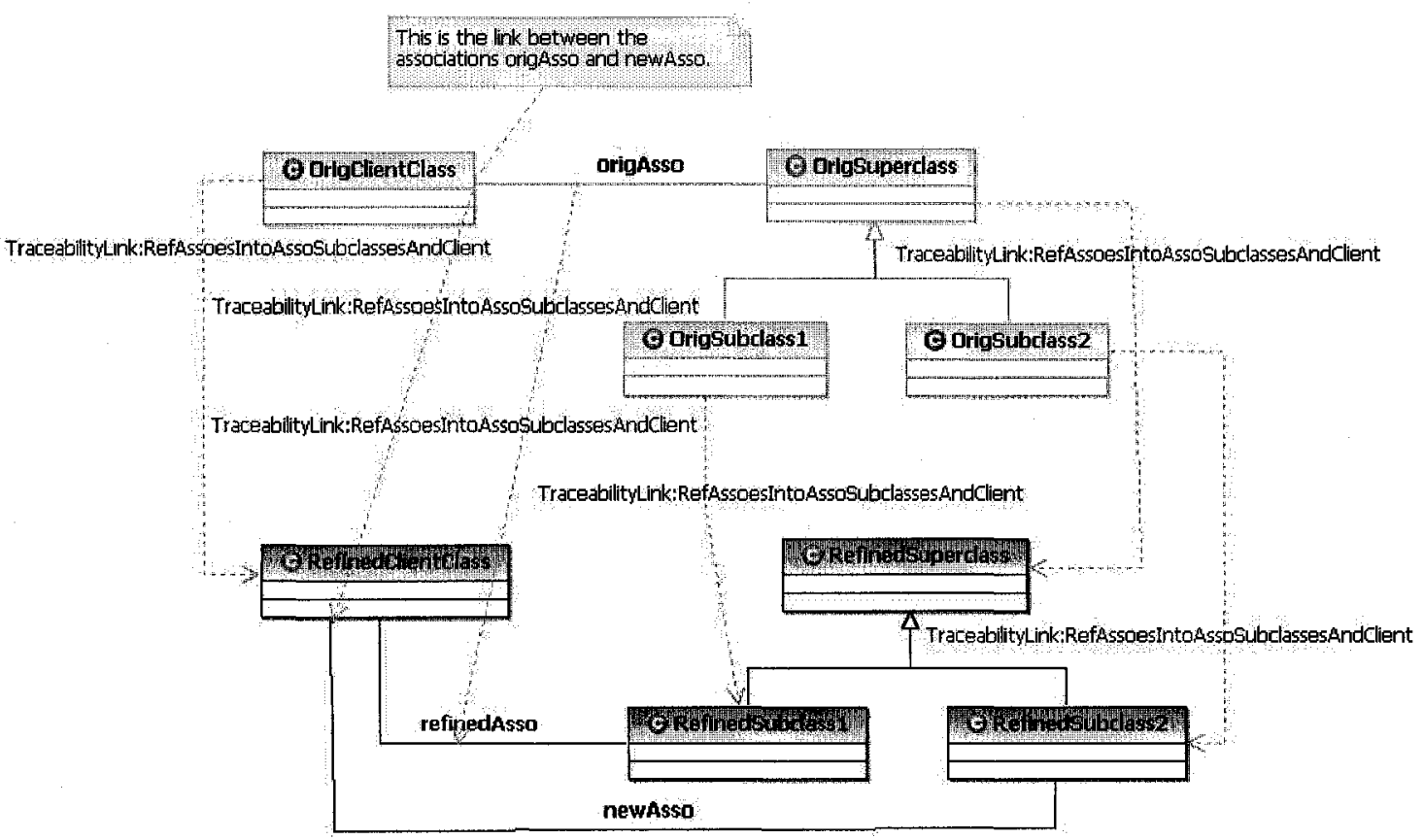

Figure 112 RefAssoIntoAssoesSubclassesAndClient - Specification 
User's Intention: The association between a client class and a superclass can be specialized to the association between the client class and one of subclasses of this superclass.

Atomic Changes: Two kinds of atomic changes are used to derive the refinement: RelocateAssociation and AddedAssociation. Note that it is possible that the original association between the client class and the superclass is deleted (a DeletedAssociation atomic changes) and a new association between the client and a subclass is added. We assume the former situation. The specification is shown in Figure 113.

Traceability Links: Six traceability links have to be established:

- one between the classes Origsuperclass (referred to as origsuper) and RefinedSuperclass (referred to as refinedsuper);

- one between the classes origsubclass1 (referred to as origSub1) and RefinedSubclass 1 (referred to as refinedSub1);

- one between the classes origsubclass2 (referred to as origsub2) and RefinedSubclass2 (referred to as refinedSub2);

- one between the classes OrigClentclass (referred to as origclient) and Refinedclentclass (referred to as refinedclient);

- one between the association origAsso (referred to as origAsso) and the association refinedAsso (referred to as refinedAsso).

- one between the association origAsso and the association newAsso (referred to as newAsso).

These traceability links are specified in the OCL expressions in Figure 114.

\section{User Help: none.}




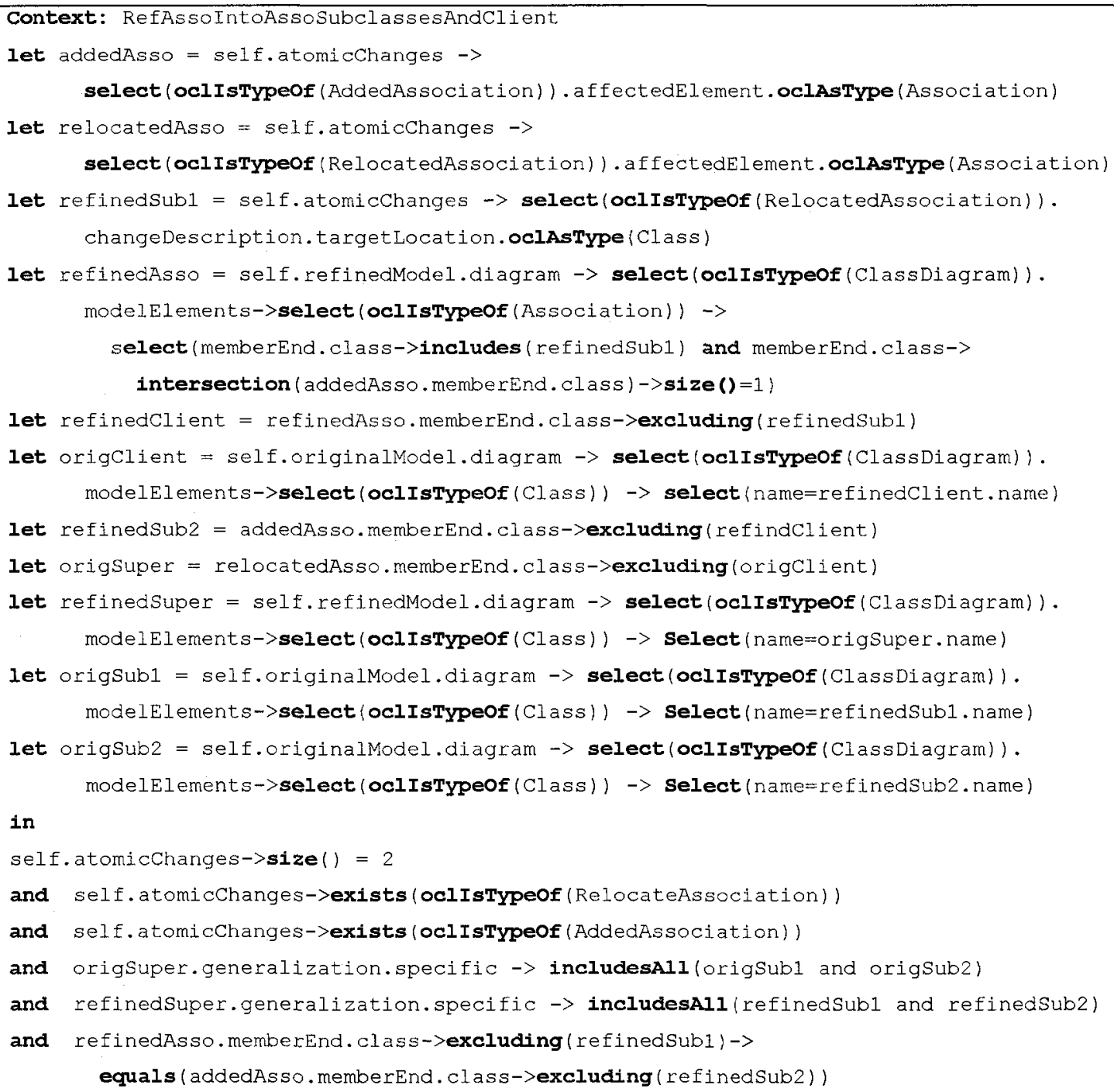

\section{(a) Constraint on atomic changes}

Figure 113 Refinement RefAssoIntoAssoes SubclassesAndClient (part A) 


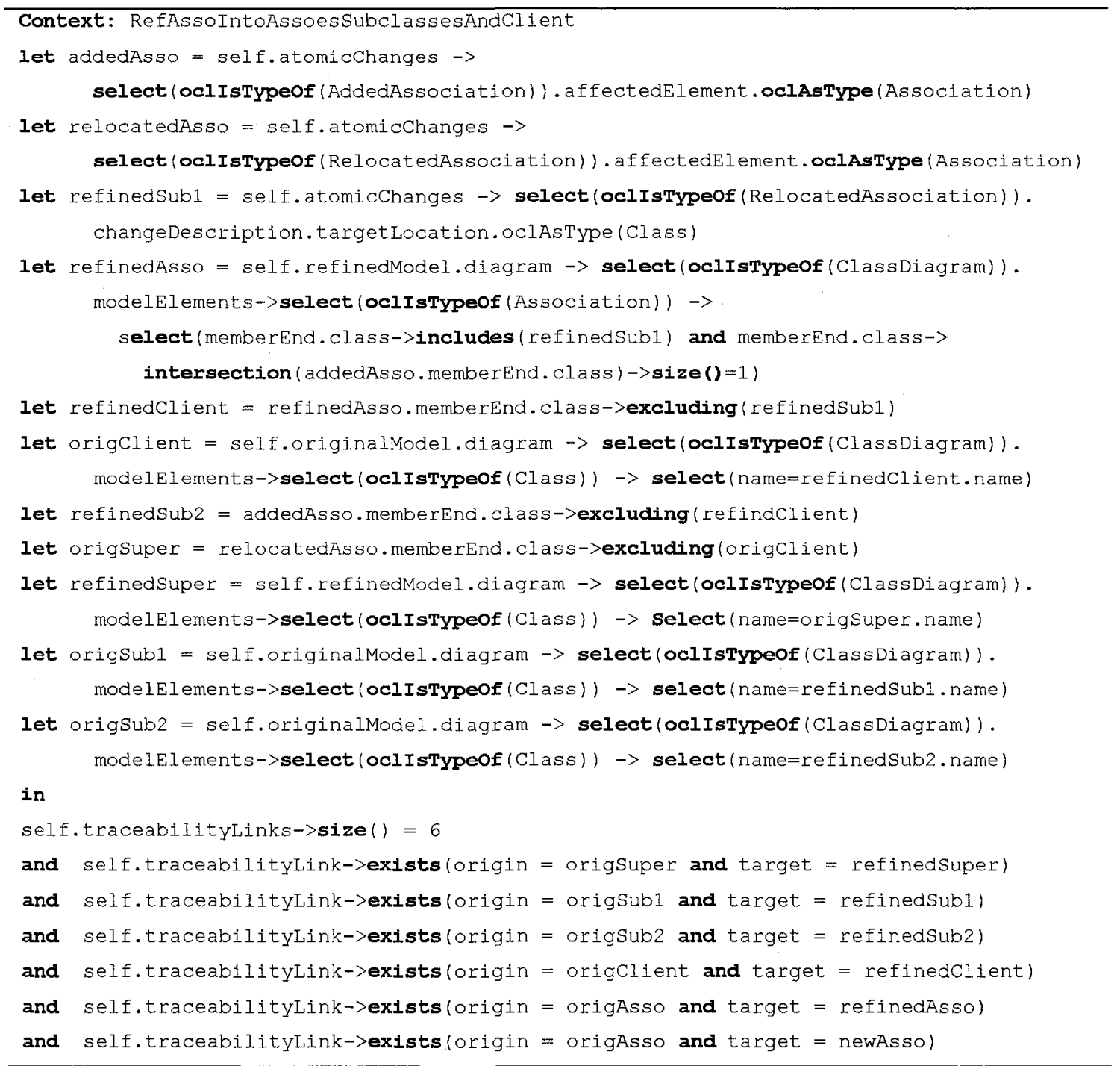

\section{(b) Traceability links}

Figure 114 Refinement RefAssoIntoAssoessubclassesAndClient (part B) 


\section{E.8 InterfaceRefinement}

Any refinements related to interfaces belong to this type of refinement. Figure 115 provides a refined classification involving three types of refinements.

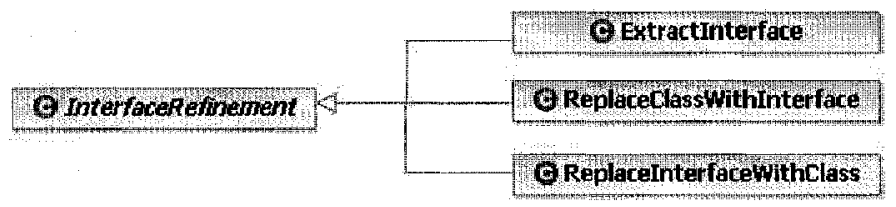

Figure 115 Taxonomy for Refinements of Type InterfaceRefinement

ExtractInterface is to add an interface and let the class being refined to implement this interface (Section E.8.1). ReplaceclasswithInterface is to replaces the class being refined with an interface (Section E.8.2). ReplaceInterfacewithclass is to replace the interface being refined with the superclass of a class which has implemented the interface (Section E.8.3). 


\section{E.8.1 ExtractInterface}

Description: Adds an interface and lets the class being refined to implement this interface.

Specification: As shown in Figure 116, the class Originalclass is refined into the class RefinedClass which implements the interface Interface.

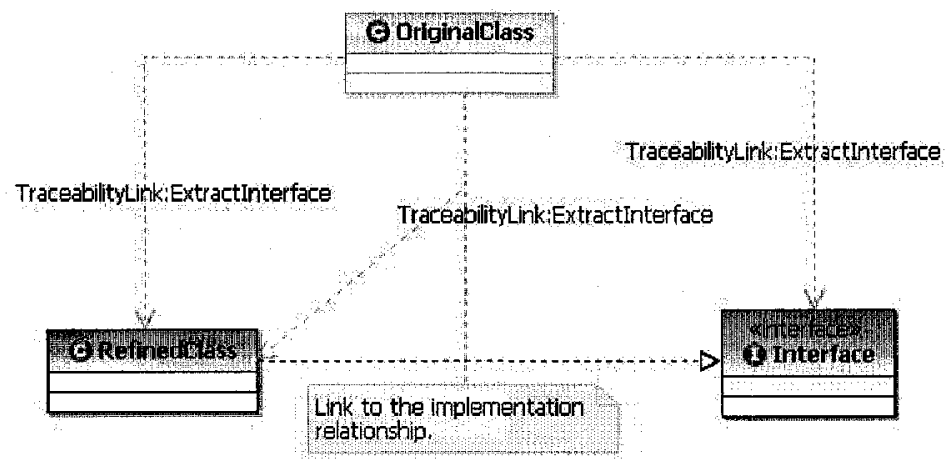

Figure 116 ExtractInterface - Specification

User's Intention: A subset of a class's responsibilities can be extracted out from this class to an interface so that these responsibilities can be shared by other classes.

Atomic Changes: Two kinds of atomic changes are used to derive the refinement: AddedInterface, AddedDependency. The specification is given in Figure 117 (a).

Traceability Links: Three traceability links have to be established: one between the classes Originalclass (referred to as origclass) and Refinedclass (referred to as refinedClass); one between the classes Originalclass and the interface realization relationship (connecting the class RefinedClass and the interface Interface) (referred to as realizationRel); one between the classes Originalclass and the new added interface Interface (referred to as interface). These traceability links are specified in the OCL expressions in Figure 117 (b).

User Help: none. 


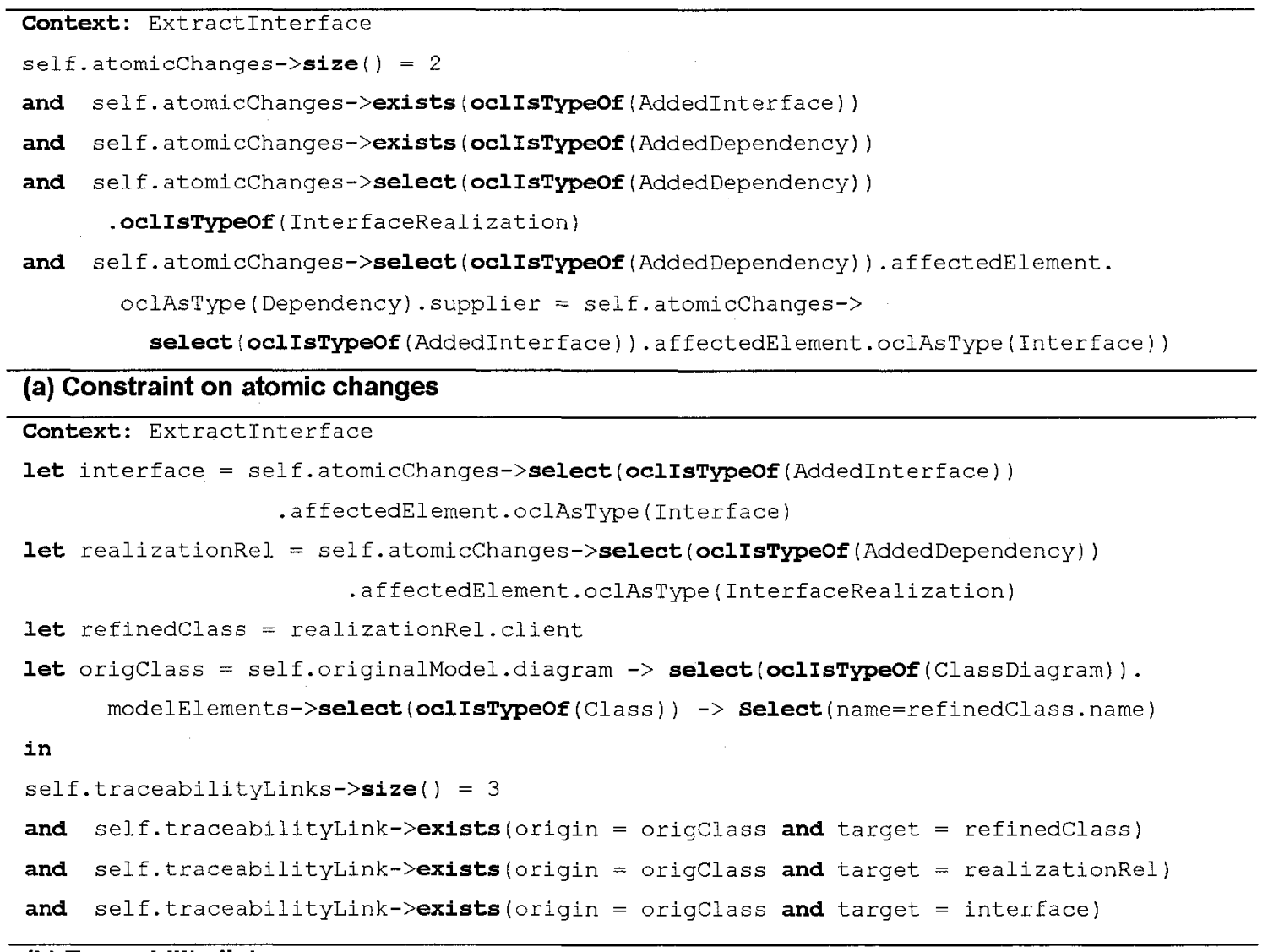

(b) Traceability links

Figure 117 Refinement ExtractInterface 


\section{E.8.2 ReplaceClasswithInterface}

Description: Replaces the class being refined with an interface.

Specification: As shown in Figure 118, the class Originalclass is replaced by the interface Interface. The operation operation 1() is moved into the interface.

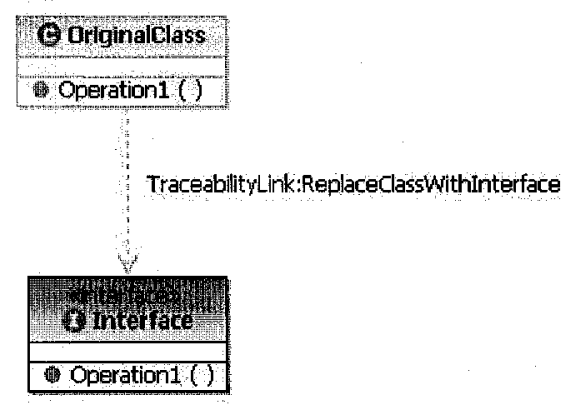

Figure 118 ReplaceclasswithInterface - Specification

User's Intention: The modeling situation would be more appropriate for interface instead of class.

Atomic Changes: Three kinds of atomic changes are used to derive the refinement: AddedInterface, DeletedClass and Movedoperation. Note that the atomic change Movedoperation may appear more than once since this case is not shown in the specification given in Figure 119 (a).

Traceability Links: One traceability link has to be established: one between the class Originalclass (referred to as origclass) and the interface Interface (referred to as interface). The traceability link is specified in the OCL expressions in Figure 119 (b).

User Help: none. 


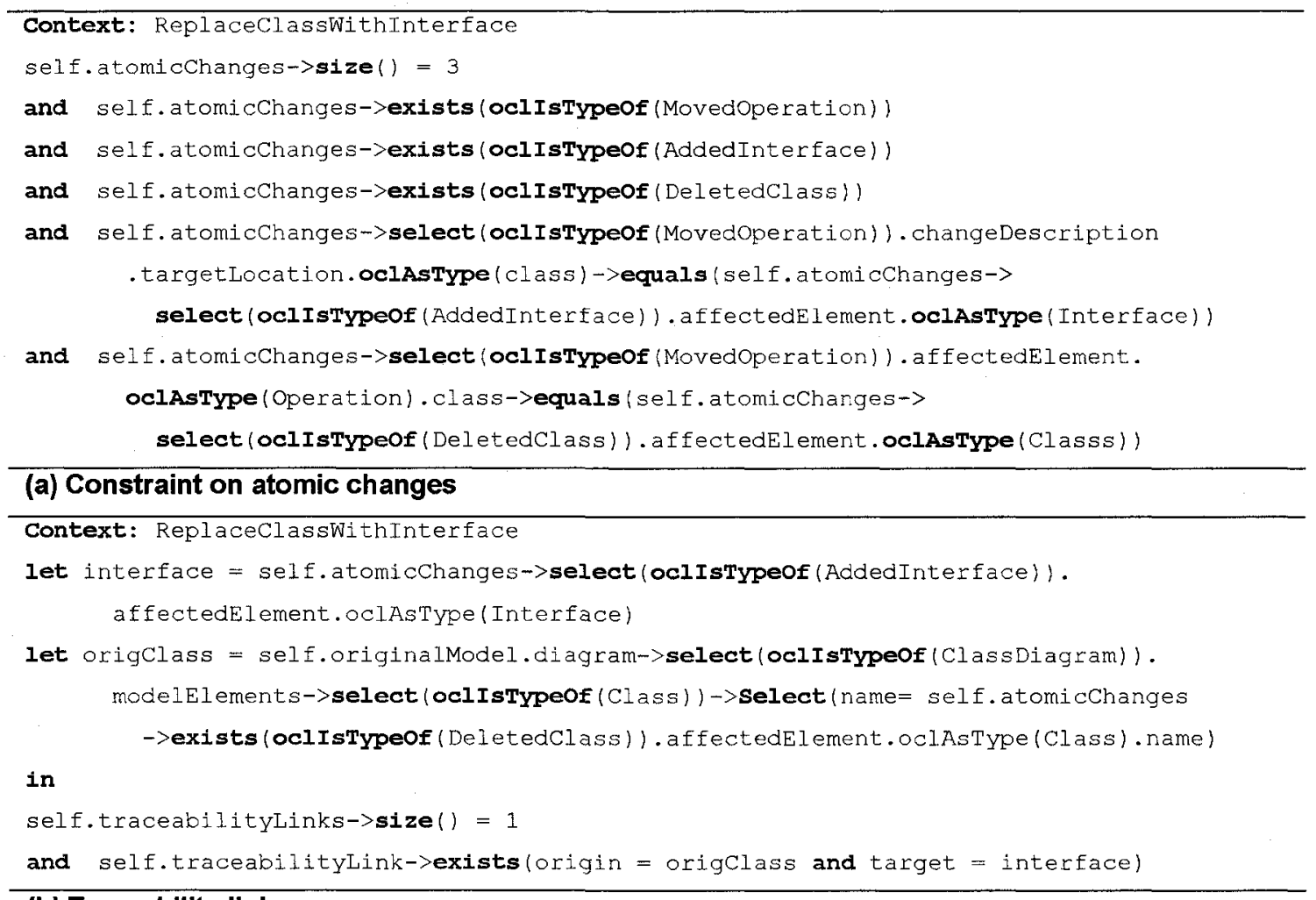

(b) Traceability links

Figure 119 Refinement ReplaceclassWithInterface 


\section{E.8.3 ReplaceInterfaceWithsuperclass}

Description: Replaces the interface being refined with the superclass of a class which has implemented the interface.

Specification: As shown in Figure 120, the interface OrigInterface is refined into the superclass superclass which generalizes the class Refinedclass. The class Refinedclass is refined from the class Origclass which implements the interface origInterface. The operation operation 1() is moved from the interface OrigInterface into the superclass.

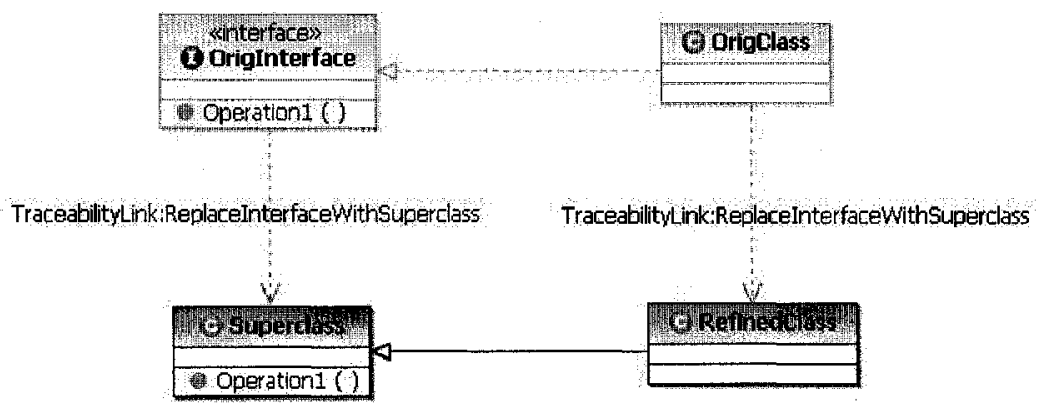

Figure 120 Replace InterfaceWithSuperclass - Specification

User's Intention: The situation would be more appropriate for superclass instead of interface.

Atomic Changes: Five kinds of atomic changes are used to derive the refinement: DeletedInterface, AddedClass, DeletedDependency, AddedGeneralization and Movedoperation. Note that the atomic change Movedoperation may appear more than once since this case is not shown in the specification given in Figure 121 (a).

Traceability Links: Two traceability links have to be established: one between the classes origclass (referred to as origclass) and Refinedclass (referred to as refinedClass); one between the interface origInterface (referred to as origInterface) and the superclass Superclass (referred to as superclass). These traceability links are specified in the OCL expressions in Figure 121 (b). 
User Help: none.

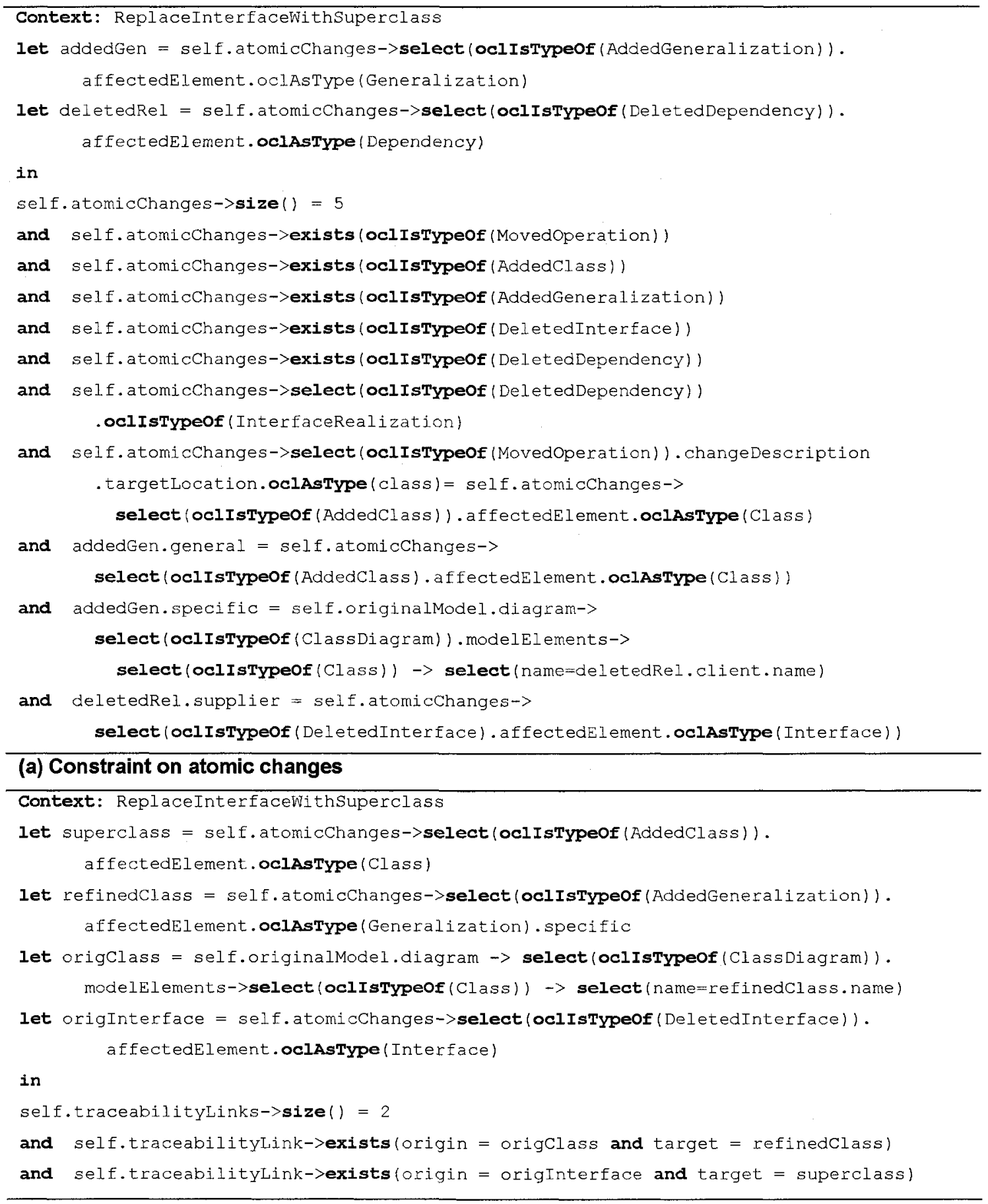

(b) Traceability links

Figure 121 Refinement ReplaceInterfaceWithSuperclass 


\section{Appendix F Formalization of Path}

Class Path does not appear in our metamodel as it is only a helper class used to shorten our OCL expressions when we formalize refinement kind Rel->ClassestRels. As shown in Figure 122, a Path instance is linked to a sequence of Elements instances, i.e., a path is a sequence of UML class diagram model elements. Notice that a path is the aggregate of at least two model elements which indicate the start and end of the path.

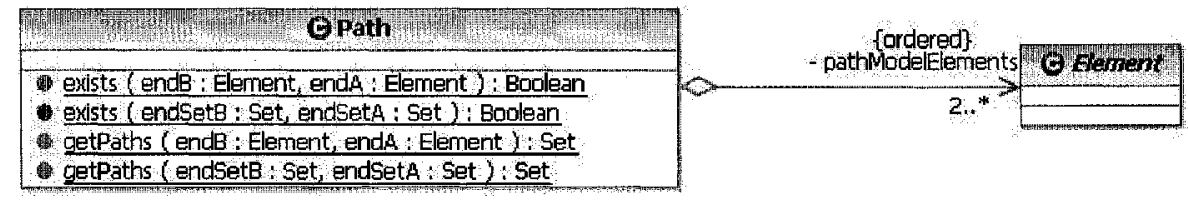

Figure 122 The metamodel for Path

Since a Path instance is the aggregate of a sequence of UML model elements in the class diagram, these model elements should be associated together one by one. For example, if the start element is an instance of Classifier, then the next element should be an instance of Relationship. Otherwise, if the start element is an instance of Relationship, then the next element should be an instance of classifier. In other words, in the context of class Path, we can write the following OCL constraints as its invariant.

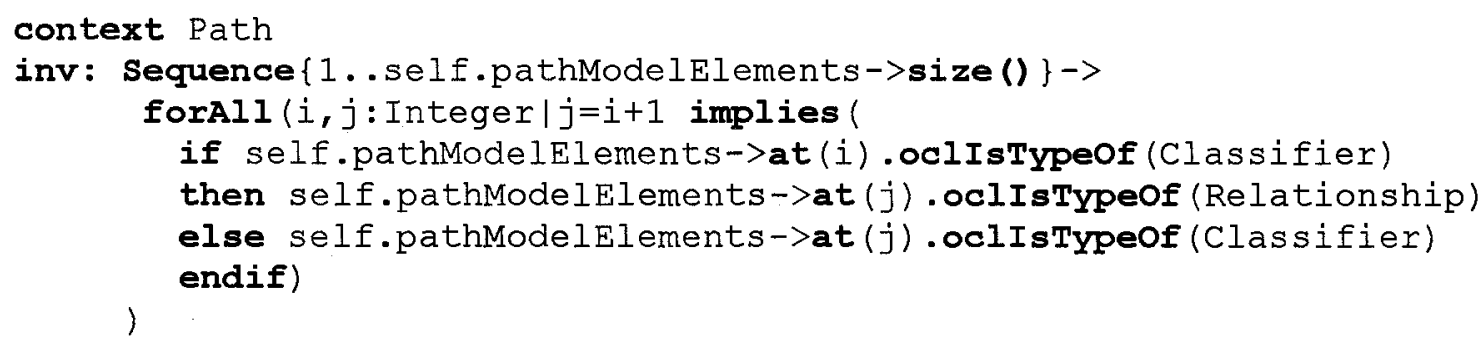

Class Path has four static operations: exists (endA, endB), exists (endSetA, endSetB), getPaths (endA, endB), and getPaths (endSetA, endSetB), which are specified and formalized as follows. 
1. exists (endA, endB)

- Description: this operation is used to check whether there exists a path between model elements endA and endB in the class diagram. It returns true if and only if there exists such a path.

- Return type: boolean.

- Parameters: endA and endB are instances of Element.

- Formalization:

context Path: :exists (endA, endB):Boolean

pre: endA->notempty () and endB->notEmpty() and endA.oclIsTypeof (Element) and endB.oclIsTypeof (Element)

post: result $=($ self.getPaths $(e n d A$, endB) $->$ size ()$>0)$

2. exists (endSetA, endSetB)

- Description: this operation is used to check whether there exists a path between any pair of model elements, each element in the pair belonging to one of the two sets: endSetA and endSetB. Return true if and only if there exists a path between any pair of model elements.

- Return type: boolean.

- Parameters: endSetA and endSetB are sets.

- Formalization:

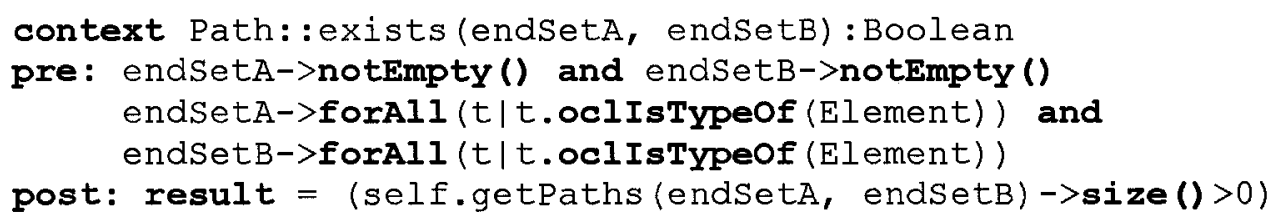

3. getPaths (endA, endB)

- Description: this operation returns all the paths between the model elements endA and endB.

- Return type: instances of class Path. 
- Parameters: endA and endB are instances of Element.

- Formalization:

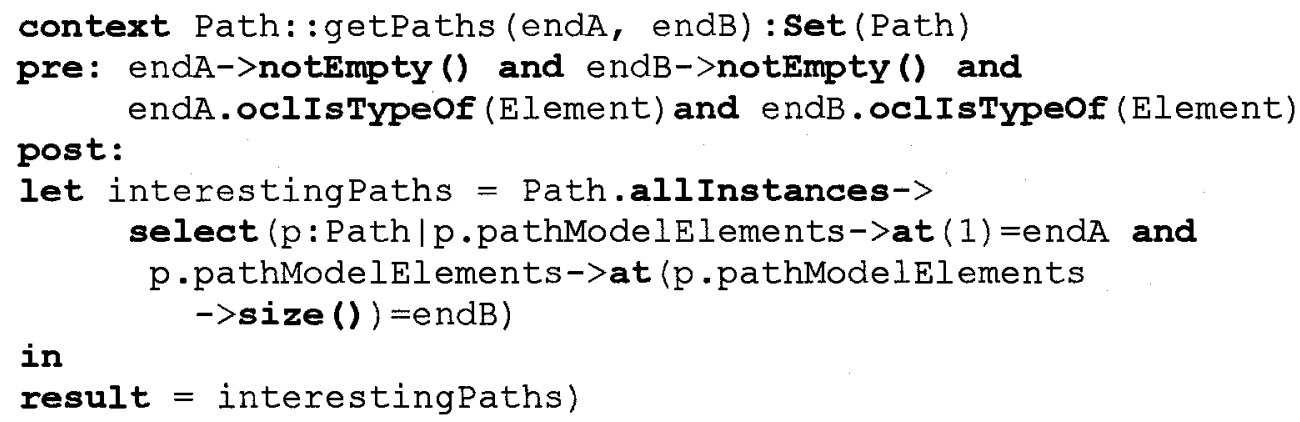

4. getPaths (endSetA, endSetB)

- Description: this operation returns all the paths between any pair of model elements, each element in the pair belonging to one of the two sets: endSetA and endSetB.

- Return type: paths which are composed of a sequence of model elements.

- Parameters: endEndA and endEndB are sets.

- Formalization:

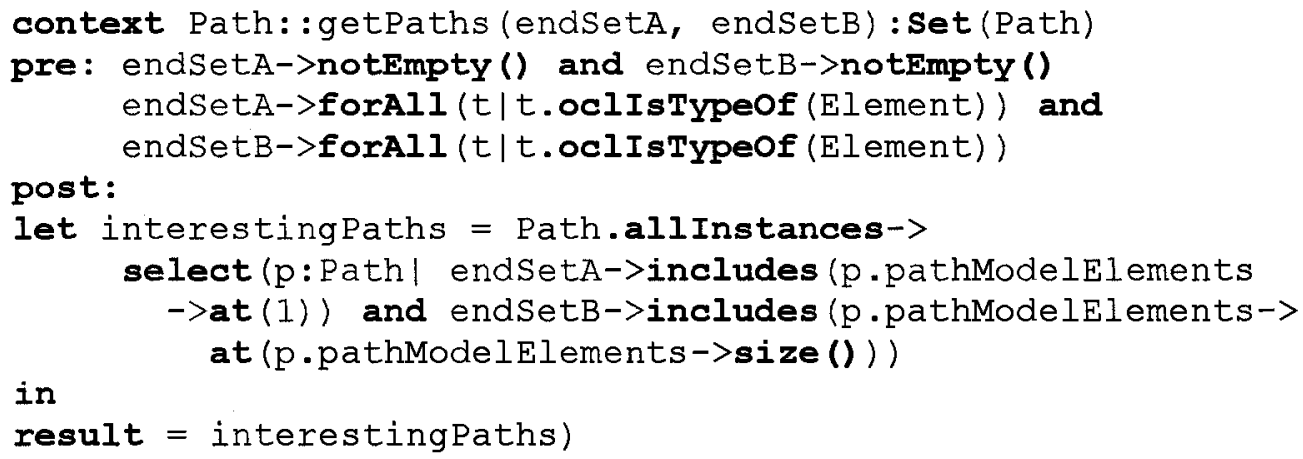




\section{Table of Contents (complete)}

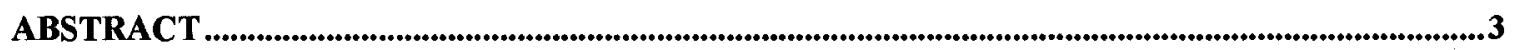

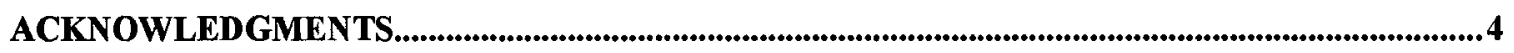

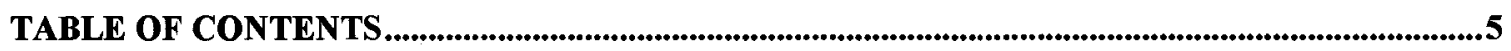

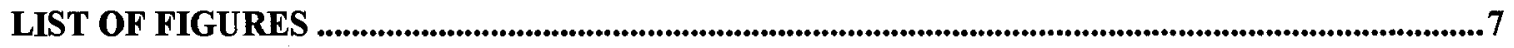

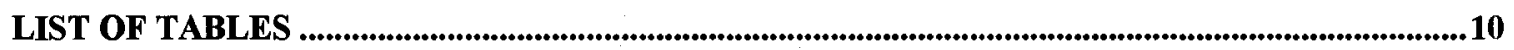

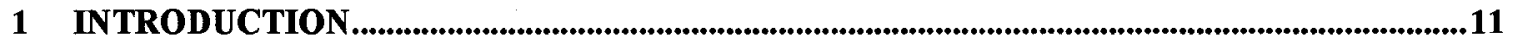

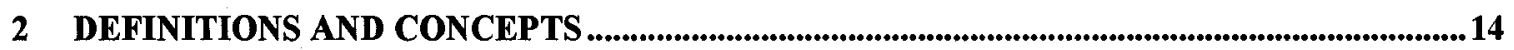

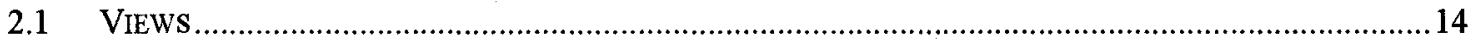

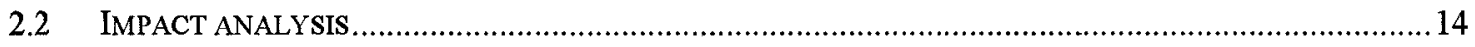

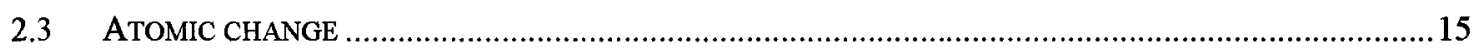

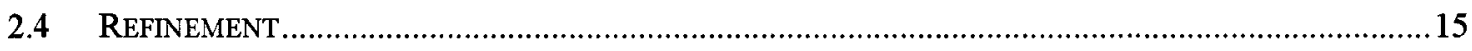

2.5 TRACEABILITY AND TRACEABILITY LINK .................................................................................. 16

3 STATE OF THE ART .........................................................................................................................17

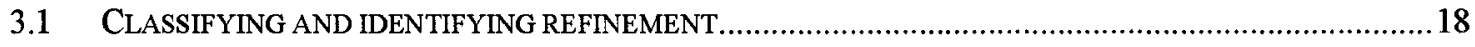

3.1.1 Mens et al. - Automating Support for Software Evolution in UML .................................... 18

3.1.2 Engels et al. - Consistency-Preserving Model Evolution through Transformation.................. 19

3.1.3 Shen et al. - Extending the UML Metamodel to Support Software Refinement........................ 20

3.1.4 Hnatkowska et al. - Refinement relationship between collaborations......................................21

3.1.5 Pons and Kutsche - Traceability Across Refinement Steps in UML modeling.........................22

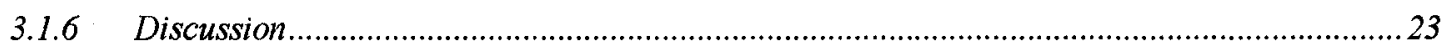

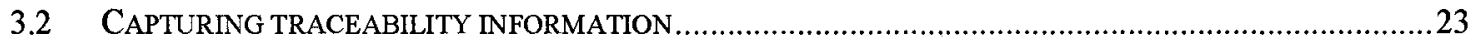

3.2.1 Letelier - A Framework for Requirements Traceability in UML-based Projects..................... 23

3.2.2 Judson et al. - Supporting Design by Pattern-based Transformation ...................................... 24

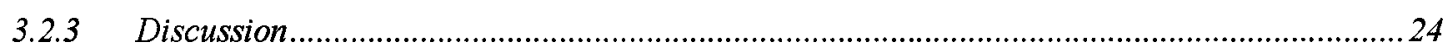

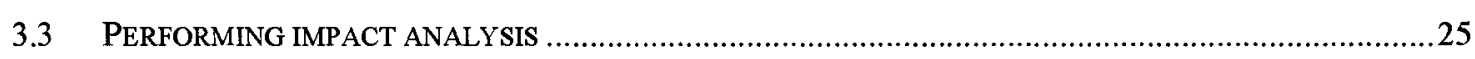

3.3.1 Briand et al. - Automated Impact Analysis of UML Models..................................................25

3.3.2 Li and J. Offutt -Algorithmic Analysis of the Impact of Changes to Object-Oriented Software 26

3.3.3 Mens and D'Hondt - Automating Support for Software Evolution in UML..........................28

3.3.4 von Knethen et al. - QuaTrace: A Tool Environment for (Semi-)Automatic Impact Analysis

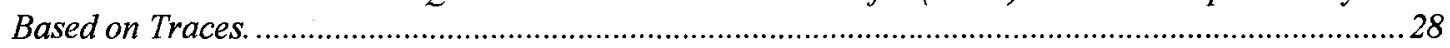

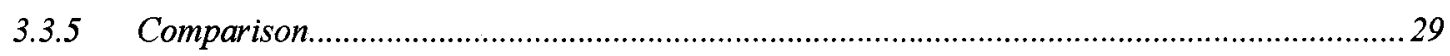

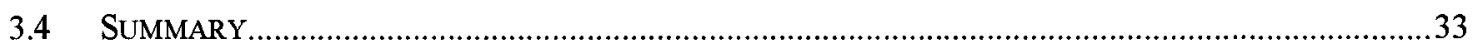

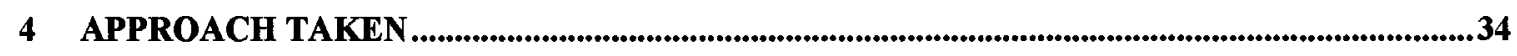

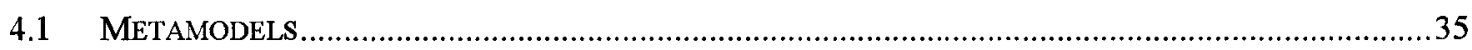

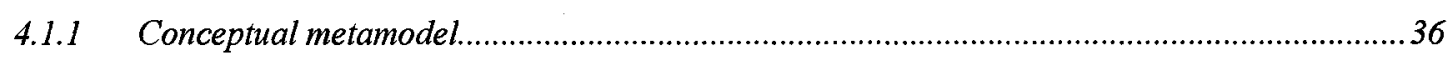

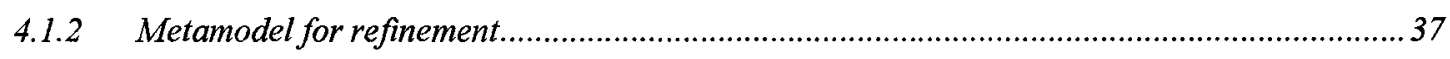




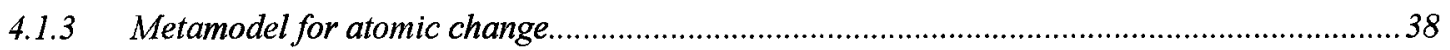

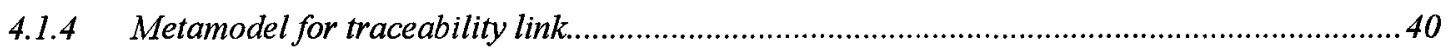

4.1.5 Metamodel for performing vertical impact analysis............................................................ 43

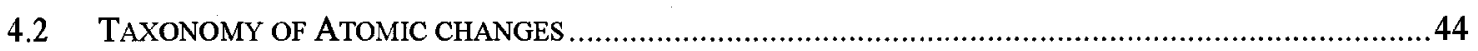

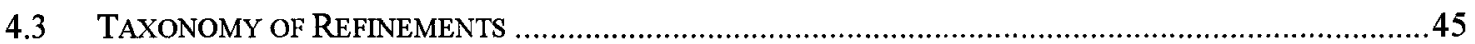

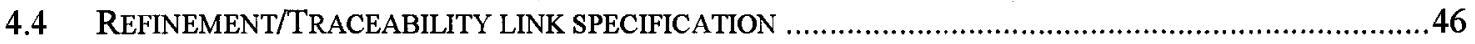

4.4.1 Atomic refinements derived from a single atomic change ...................................................46

4.4.2 Atomic refinements derived from several atomic changes..................................................48

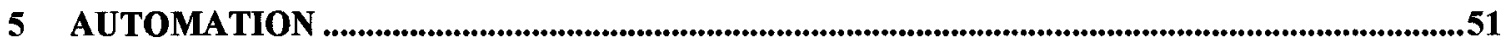

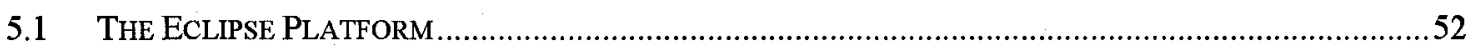

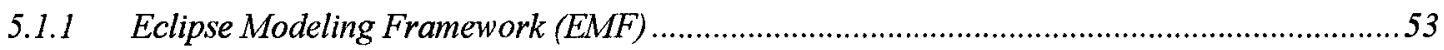

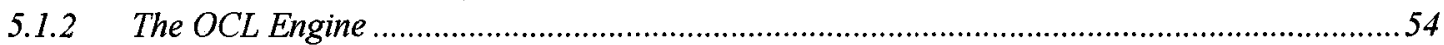

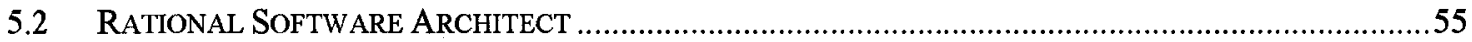

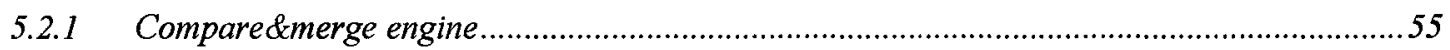

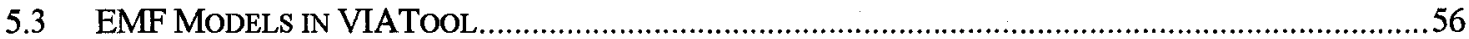

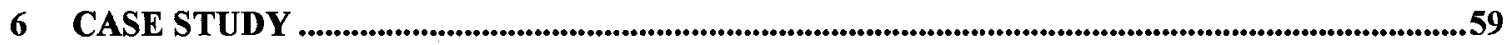

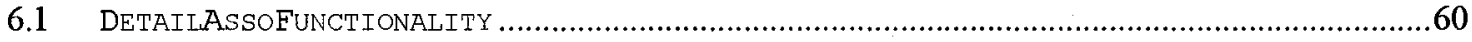

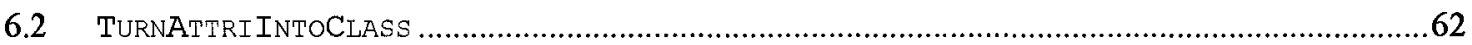

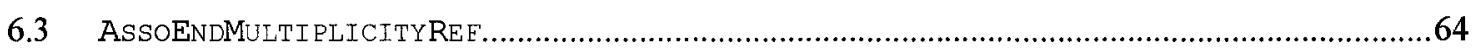

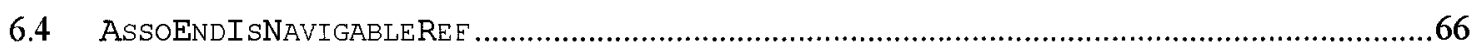

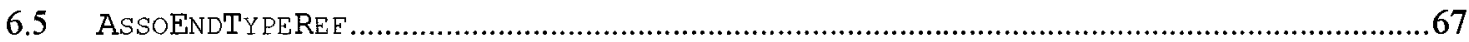

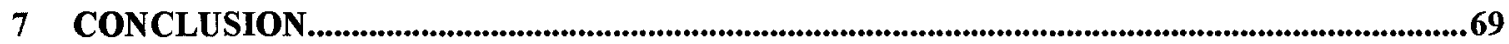

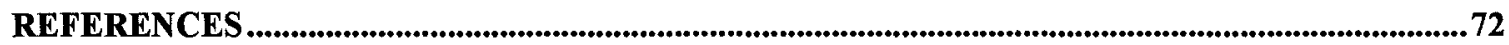

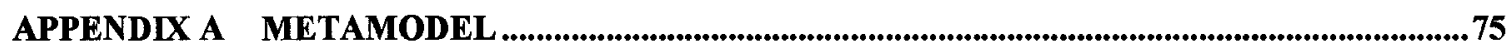

APPENDIX B DICTIONARY FOR METAMODEL ................................................................................76

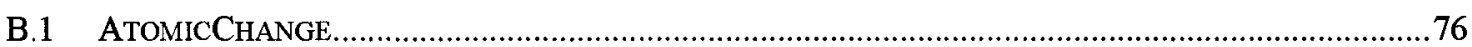

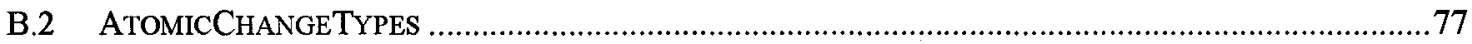

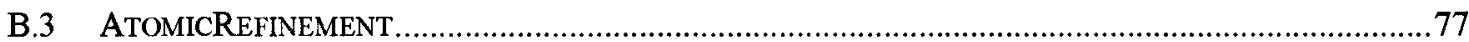

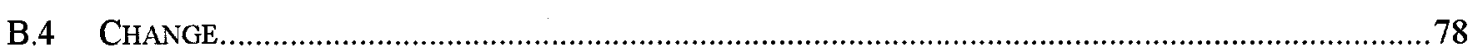

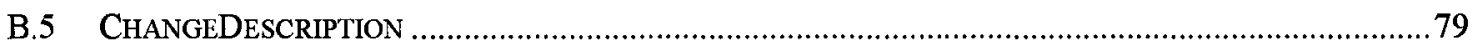

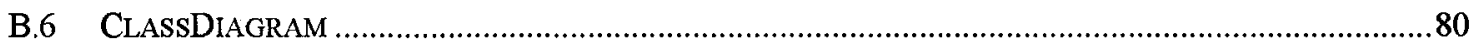

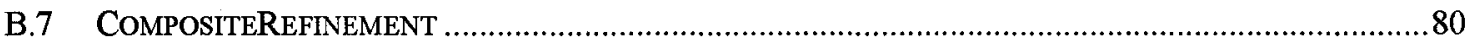

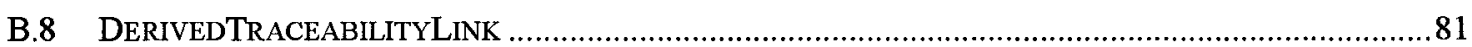

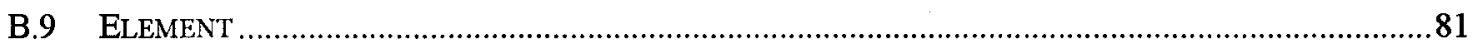

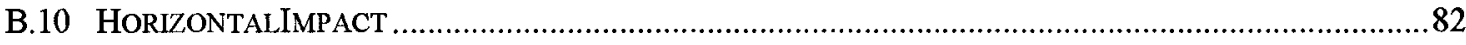

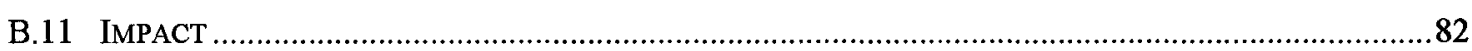




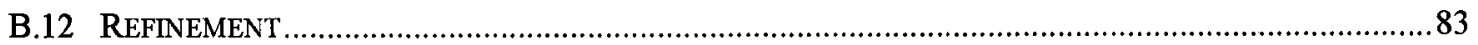

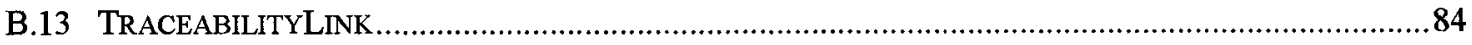

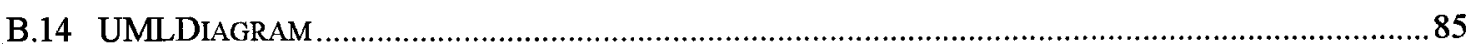

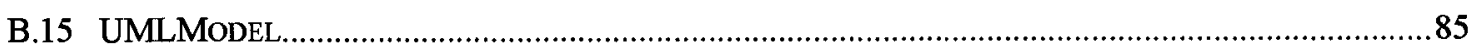

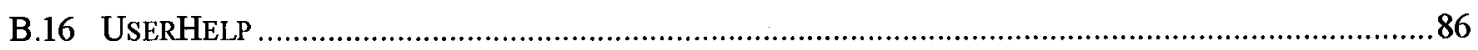

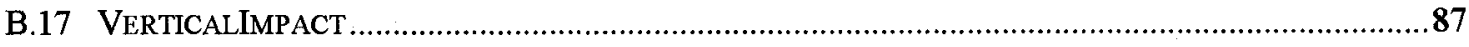

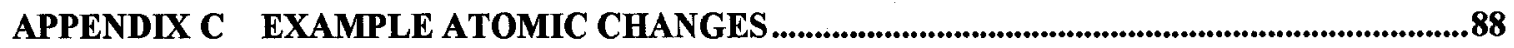

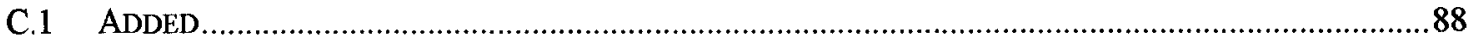

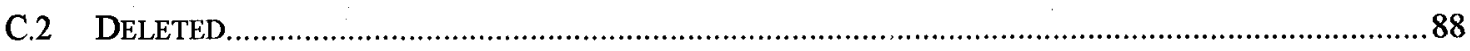

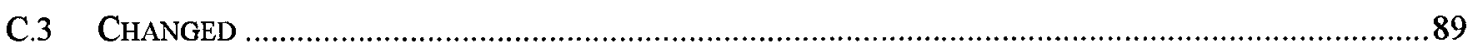

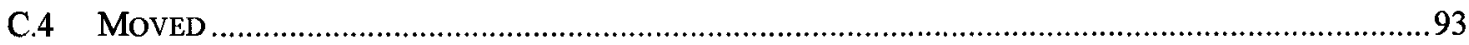

APPENDIX D TAXONOMY FOR ATOMIC CHANGES ..........................................................95

APPENDIX E TAXONOMY AND FORMALIZATION FOR REFINEMENTS ..........................101

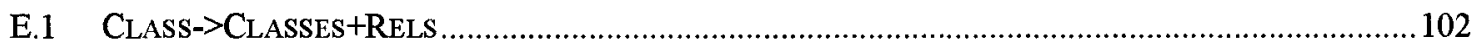

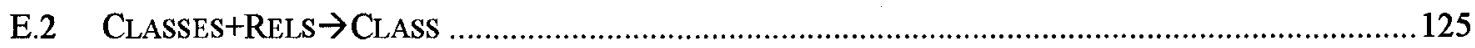

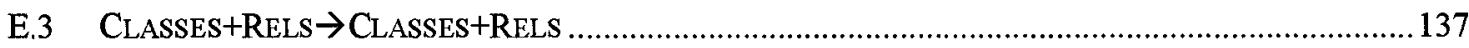

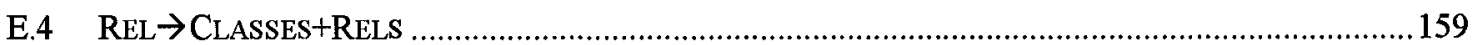

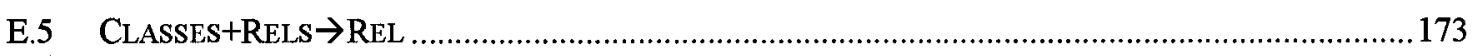

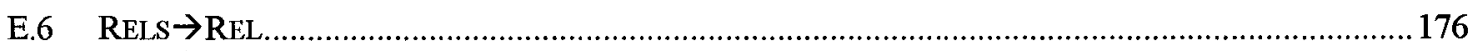

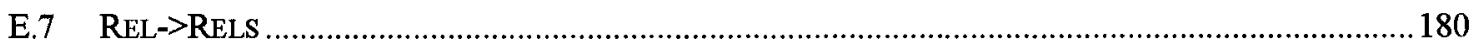

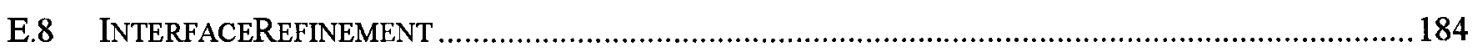

APPENDIX F TORMALIZATION OF PATH................................................................. 191 